5)

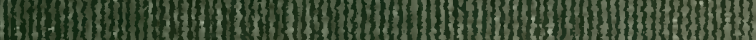
(15) My

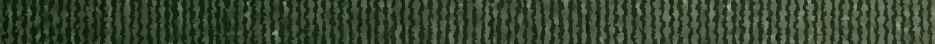
Ify \{is

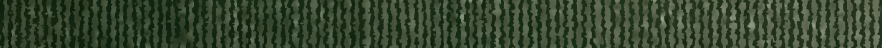
14) IIII) II 3.

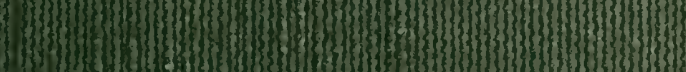

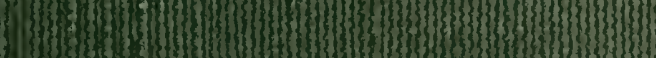

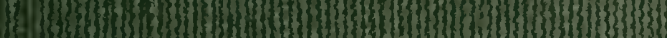

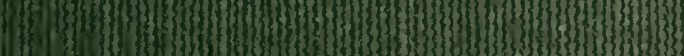
(3) S)

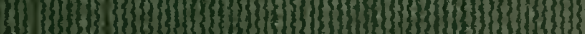

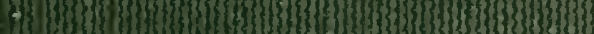
E⿺廴⿻肀二𠃋

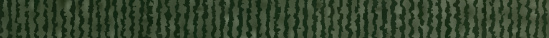

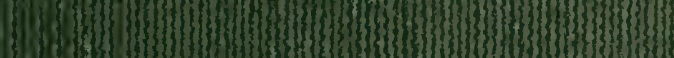

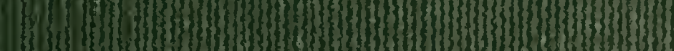
13

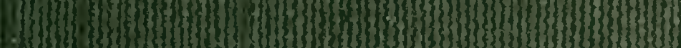
E⿺廴⿻肀二𠃋

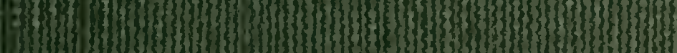

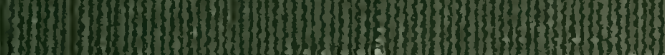
III

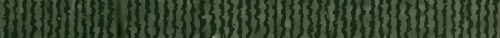

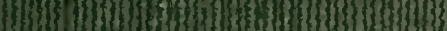

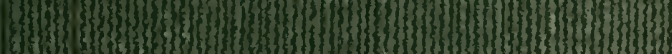
III पा 4)

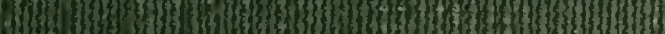

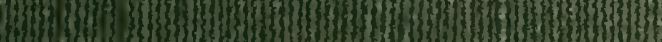
W.

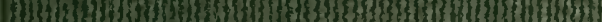
4. (4)

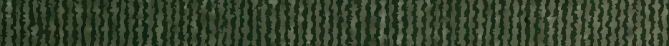

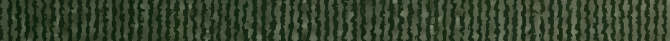
(9) tII-1) \{1 dilys

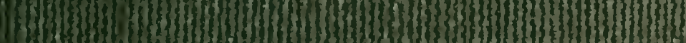
5. nim H IIII) Hits

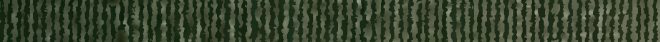


IfI

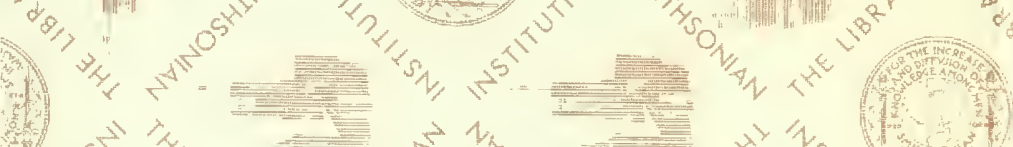

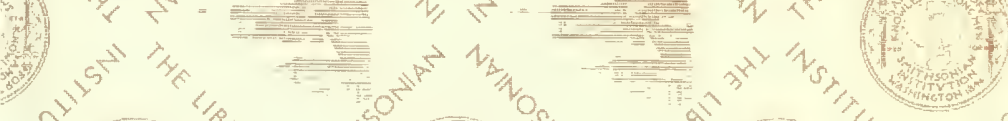
ond

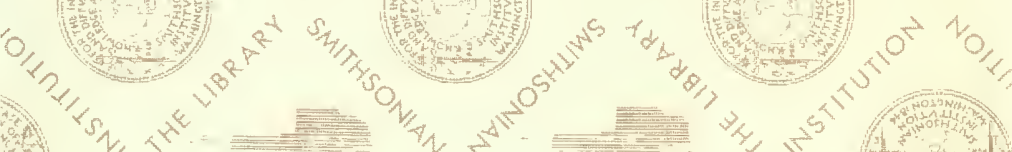
2,2
1

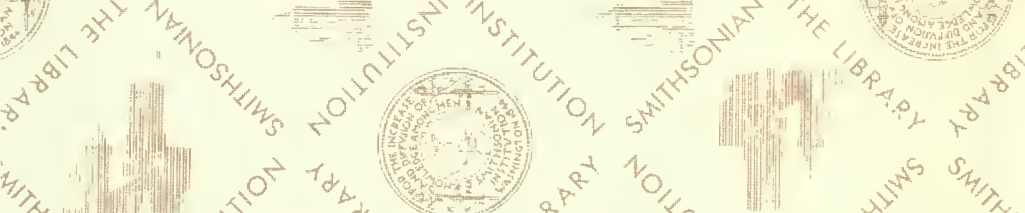

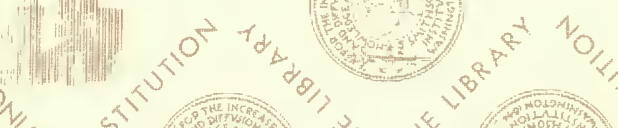

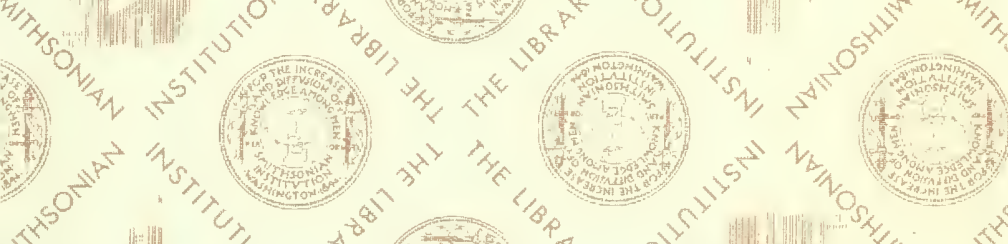

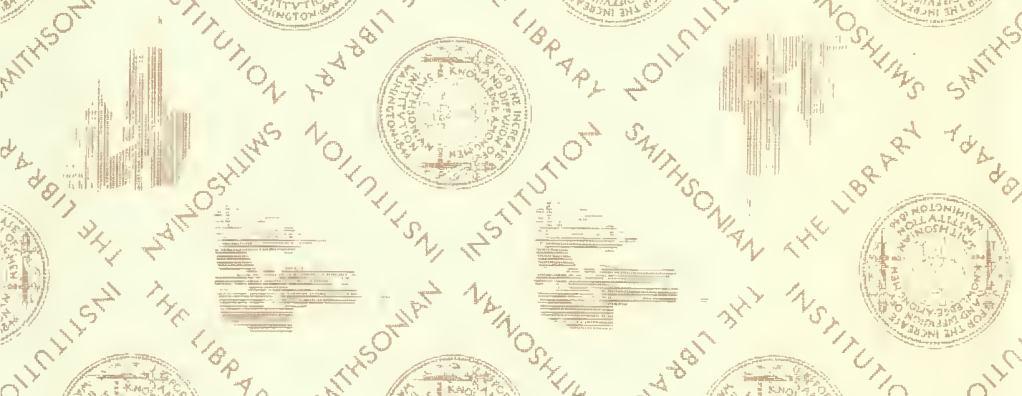

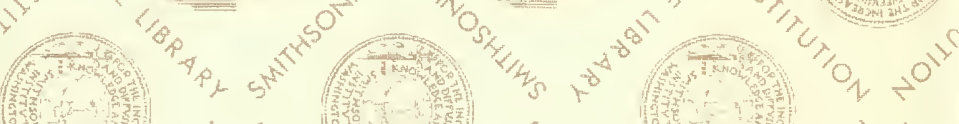

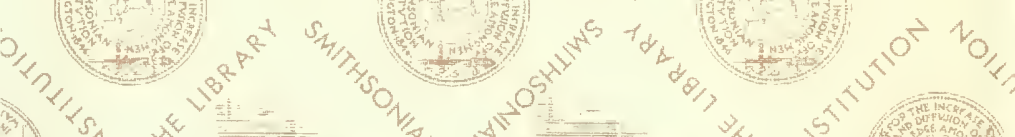
s.t.

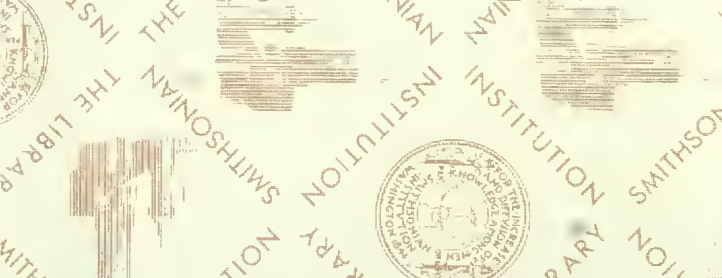

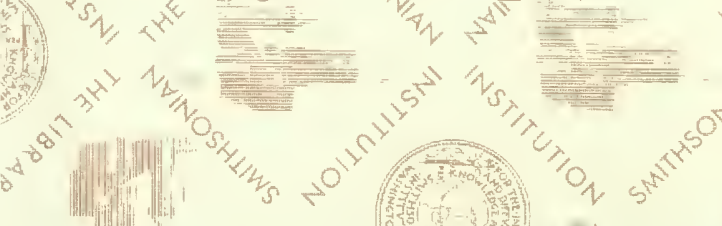
Win

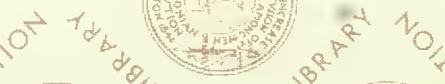



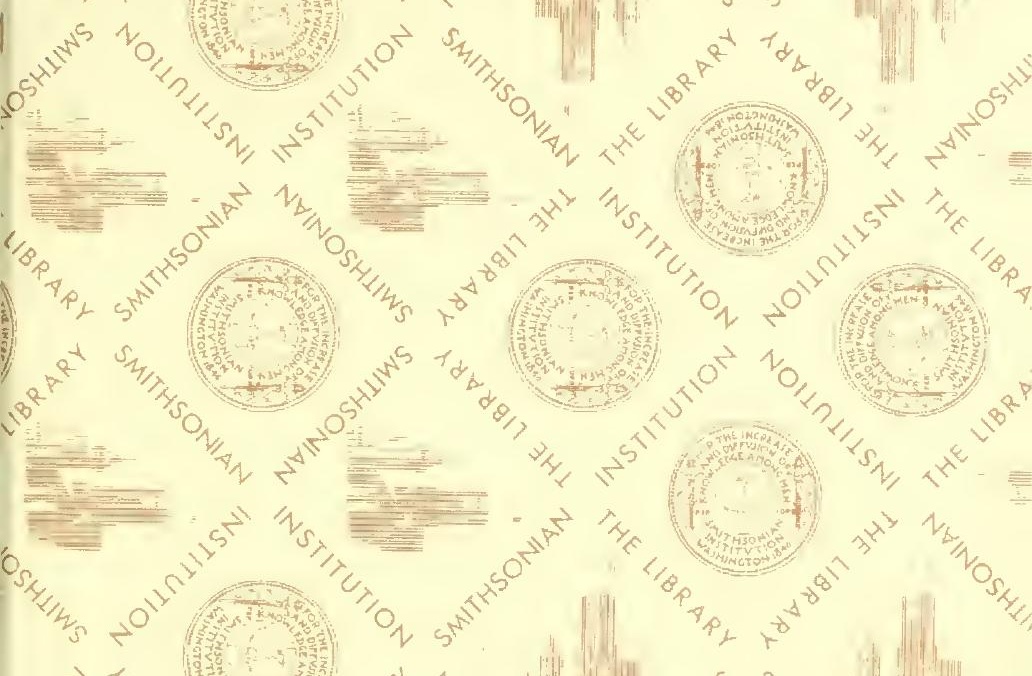

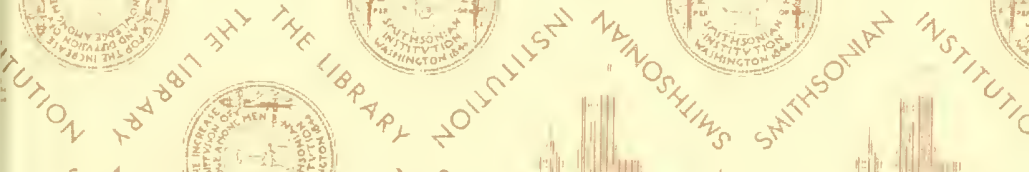

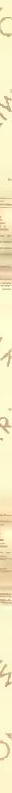


C 

Collatisn of kid. . .

$8: 1^{5}, 2-14^{3} ; 15^{7}[\$ 1 ;(-1)$ sigret $] ;$

2 misprivited 9 (this copis but not a.1)

4 misprinted $2, f f .116$,

This copt lacks:

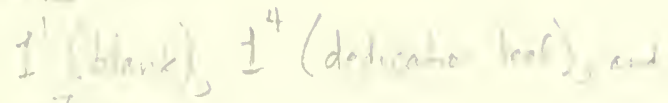

$$
\begin{aligned}
& 15^{7} \text { (boir). }
\end{aligned}
$$

Mathile H, ve 


\title{
DIPTEROLOGIN ITHLCE
}

\author{
PRODROMUS
}

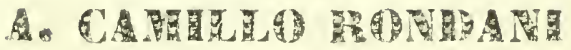

$$
\text { Vor: } \mathbb{1} \text {. }
$$




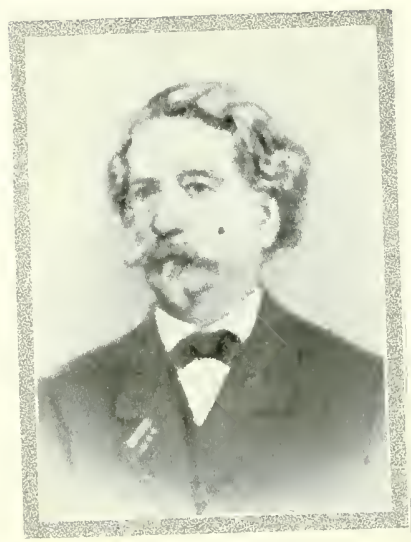

c. Rondani. 


\section{GENERA ITALICA}

\section{ORDINIS DIPTERORUII}

ORDINATIM DISPOSITA ET DISTINCTA

ET IN FAMILIAS ET STIRPES AGGREGA ${ }^{\top} A$

\section{ADCTORE}

\section{CA}

IN R. Atheneo Parmensi Professore, Societatis Evrorologice Parisiensis - R. Agcademie Georgofilorem floaentika - Societatis Zoologice et Botanice Windebenersis - Accaderize Nature guriosorua Neapolitana -Societatis Entorologice Stetimensis, et Rer Agraria Regil LEPIDI SODALE ETC. ETC.

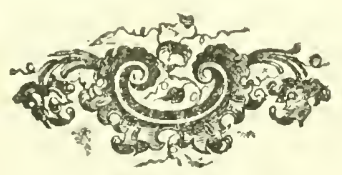

PARM

EX TIPOGRAPHIA ALEXANDRI STOCCH

옹․ 
Poi distintipi di Genere, la prima condizione d'ogni carattere, sarà quella di essere facilmente accessibile

\section{SPINOLA}

(DEI PRIONITI.) 
O uesto quadro generale sistematico dei generi Ilaliani degli Inselli Dilleri, preceduto da una classificazione delle Famiglie di quest ordine entomologico, e da quella delle stirpi europèe di rui composta ciascuna famiglia, $\dot{c}$ destinato a far parte dei materiali che devon essere accumulati per la costmaione del grande edifizio di cui tracristre le fondanenta il Principe Bonaperte di Camono coi suoi larori suliu Faunu Inalica.

Egli è periai rhe ho adoutalo per le pasole sistematiche che spetiano alle nomenclutura distinlica la slessa desinensu che egli adothava per le sile fumiglie e soltofumiglie Zoologiche; e solamente yer poco ho aleviato dalle traccie seynate dat srande naluralista roll' usare il nome di stivpe "n exee di quello di sullofomighlia col quate cyli lua distinto le divisioni primarie delle sue famiglie. La pubblicazione di questo mio lavoro i un programme, o per meglio dire is ama proposta,

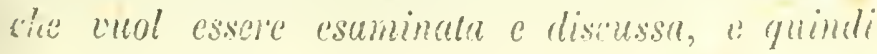


riformata dietro le considerazioni dei dolli in tade materia, e dovià poi essere seguita da uno species ditterologico dell' Italia, allorchè le discussioni avranno mostrato quanto vi $\dot{e}$ da togliere in tale progetlo.

Fra $i$ generi che trovansi qui sehicrati ve ne ha qualcuno di cui non fu ancor riscontrata sressuna specic nel nostro paese, ma ho creduto conveniente di dargli un posto fra $i$ nostrali nella persuasione che ulteriori indagini ci faranno scoprite di molti se non di tutli quei generi un qualche rappresentante in Italin, mimperoche lo differenze di clima e lo varieti di romdizioni topografiche di questa nostra regime sono tali, che dalla vetha dell' alpi alle spiagyie di Malla, dalle pendici dell' appennino alle bassure palustri delle maremme, tutte vi si possonso vitroxare le circosanze favorevoli alla vita deltr specic europie le piu disparate, tanto is cio vero che si riscontrino in diversi laoghi deb bet paese e non di rado, le specie abitarici di Barberia, e quelle della nordica Scandinavia.

Spero che gli entomologi connazionali vorranno giovarmi colla communicazione delle specialiti delle loro provincie, e cosi contribuire alla minore imperfesione dellu Faunilla Ditterotogica Italiana.

\section{Camelo Rondan.}




\section{DIP'TERA}

INSECTORUM EXAPODORUM ORDO, CUJUS GENCRA

Mandibulis maxillisque veris destituta.

Proboscide seu haustello alimental liquida sugentia. Oculis reticulatis semper instructa, ocellis adsint vel non. Antennis semper disllnctis et articulatis.

Alis duabus venosis, extensis, raro abortivis, rarissimo nullis. Halteribus sub radicem alarum semper prædita.

Uncis tarsorum apicalibus nunquam polidentatis nec pectiniformibus.

Metamorphosi completæ snbjecta.

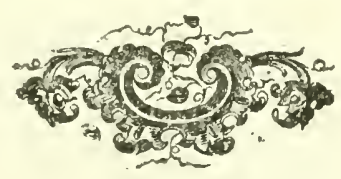




\section{ORDINIS DIP'TERORUM FAMILIAE}

\section{CHARACTERIBUS HAGIS PERSPICUIS DISTINCTA:}

A. Aalenrace articulis tribus (stilo vel arista exceptis si ardsunl), raro duobus tantun, ad summum sex manifestis, et tunc palpi articulis ad summum duobus distinctis.

B. Antennce articulo ultimo haud circulatim inciso el stiligerce vel setigerce, si vero stilus et arieta non adsunt, vel loco stili articuli aliqui supranumerum siti sunt, tunc proboscis cornea est et porrecta: rarius proboscis membranosa et brevis antennequa mutica sunt, el tunc caliptera amplissima.

C. Os fere clansum, proboscis et palpi non aut vix apparentes.

\section{FAN. I. OESTRIDA.}

C.C. Os magis vel minus late apertunı, proboscis semper, palpi sæpius distinclissimi.

D. lroboscis sæpissime brevis et membranosa, sì raro cornea el porrecta tunc arista, in dorso articuli tertii sita, aut venæ longitudinales alarum ad summum octo si apicalis rel nulla.

li. lona Inngiludinalis supranumerum spuria inter quartam. 
et quintam ordinarias ades?.

\section{FAN. II. SYRPHIDE.}

AE. Vena rulla spuria supramumerum in:e" quartam el quintum longitudinales. $\left({ }^{*}\right)$

F. Proboscis corna el elongata, si raro brevis, vel submembranoza, pedes selis zalidis destituti.

\section{Fam. III. CONOPiOE.}

FF. Proboscis brevis et membranosa, si raro cornea et elongata, tunc pecies setis validis instructi.

6. Alke uenis longiudinalibus saltem sex, sapins septern, et si raro quinque tantum vel minus, tunc proboscis a palpis non iecta, neque ad basim.

II. Ale venis longitudinalibus ad summum soptem, prim? eliamsi spuria vel brevis considarata, et analis quogne, sen wltima postica, elinnsi margini non froducta: si vena semplem, tunc arisla dorsualis.

1) Nel aumerare te vene longitudinali comincio dai lato anteriorc delle ali con quelli più breve e che si porta nll' orlo anteriore in maggior vicinanza della basa alare, e la chiamo prima: e çuesta vena è sempre messa a numero anche quando sia qualche volta o spuria od interotta: chiamo secenda qurlla vena longitudinale che melte capo nella costa dell' ala ossia nella vena costale subilo dopo la prima, la tcrza è quella che gli tiene dletro, e così seguitando a contare intorao at margino alare fono all' ultima del lato posteriore: l' ultima peró o le alime che trovansi alla radice dell' ali ael lato posteriore e che si chiamano dagli autori (axillares) ascellari, allorchè sono spurio o poco apparenti non le melto a numero e vou le considero come parta della rete venosa generale, benchè debbano essere aumerate colla altro yagudo sozo dighintameals osservabili per certo tratto di loro lugghezzs. 
1. Vena quint longiludinalis sursum fiexa et cubrtata. ... Calipteru nagis vel minus ampla, squama infera magis producia.

\section{FAM. IV. MUSCID E.}

11. Tena quiria longiludinalis recta rel subrecta usque ad apicem, si raro parum flexa, tunc caliptera parva ve? subnulla, squama infera non latiore.

5. Yena transversaria aliqua inter longitudinales adest, saltem unica prope radicem alarum.

L. Feno longindinales quinque costali $\left({ }^{*}\right)$ productas, si prima dux spatio interposito calloso subconfusa, tamen usque ad apicem manifeste divisate sunt.

\section{FAM. V. ANTHOMYDE.}

LL. Teno longitudinales घhatuor vel Ires tantum costals producte, et si prima basi duplex apice tamen unica. FAM. VI. AGROMYZIDA.

KK. Vence transwersarice sullit neque at basim alarum.

$$
\text { F.M. VII. PHORIDA. }
$$

HI. Alow ennis largitudiralinus saltem octo, prima eliamsi

(1) Non si confouda la vena costale col margine o costa delle ali; per vena costale si iutende quella vena che forma per un tratto più o men lungo il margine estremo dell' ali, che talora si ferma ne! lato anteriore e talora discende anche nel posteriore. 
spuria et brevis considerata, ế si septem distinctio tantum, tunc arista apicalis.

31. Ala venis transversariis nullis.

\section{FAн. VIII. LONCOPTERIDA.}

MM. Alo veris aliquibns transversariis.

N. Stilus antennarum apicalis.

\section{FAM. IX. PLATYPEZIDE.}

NN. Stilns antennarum in dorso articuli tertii insertus.

\section{FaM. X.PIPUNCULDDE.}

GG. Alee venis longiludinalibus quinque vel sex tantum. Palpi proboscidem saltem ad basin tegentes - Organa copulatoria maris sæpius appendicibus latis vel fimbriis longiusculis instructa.

\section{FAM. XI. DOLICOPIDA.}

DD. Proboscis cornea el non 'abiata, si nuro membranosa et labiata, tunc stilus apicalis et $\left(^{*}\right)$ venæ longitudinales alarum decem vel undecim.

0. Caliptera parva vel subnulla.

1. Vera longitidinales alarım ad summum octo.

\section{FAN. XII. EMPIDE.}

PP. Fence longitudinales alarum saltem novem.

(1) Nei generi Atherix et lbisia sembra essere dorsale in causs della ditatazione inferiore del terzo articolo delle antende. 
Q. Vene longiludinales alarum undecim: - Frons intes oculos haud valle excavala.

\section{FAR. XIII. LEPTIDA.}

QQ. Veno longitudinales alarum novem rel decem, et quando rmlecim, lunc frons inter oculos valde excavata.

R. Frons inter oculos valde excavata.

S. Ocelli distincti. - Antcmo vel stiligere vel non, sed quando stilus abest tunc articulis magnis supranumerum ad apicem destifutæ.

\section{FAM. XIV. ASILIDA.}

S5. Ocelli non manifesti. - Anteme non stiligera, el loco stili articuli cuagni supranurerum adsunt.

\section{Far. XV. MVDAGIDA.}

RR. Frons inter oculos haud valde excavata.

\section{FAR. XVI. BOMDYLDA.}

00. Calipterca sub alcas satis ampla.

\section{Fam. XVII. CYRTIDA.}

B.h. Anenno articulo tertio circulatim inciso, vel rark in articnlos aliguos distinctos diviso, si vero rarissimo integro et non sulciolato, tunc stilus abesi, proboscis hrevis et membranosa, et caliptera parra.

T. Antennarum arliculus teritus non sulciolatus circulariser, - Siylus . !llus - insertio prope orem. Far. XVIII. SCENOPINIDA. 
T6. Sncuxa articrio fertio circulatim inciso saltem ad apicem vel in articules aliquos distinctos diviso: magis ve! minus ab ore distantes, et si prope orem inserta tunc stilus adest.

2. Antennarum articulus tertius sulciolis ad summum quatuor distinctis (stilo excepto si adest) si veru salciolis magis numerosis tunc caliptera ampla, halteres saltem maxima parte tegentia.

V. Titice nulle spinulis validis sen calcaribus terminan!tbus - Caliptera parva - Halteres detecti.

\section{Fam. XIX. STRATIOMÝDA.}

PT. Tibioe saltem intermediæ calcaribus terminantibus. Caliptera ampla, halteres saltem basi tegentia.

\section{FAM. XX. TABANIDE.}

UU. Ansernce articulo tertio sulciolis saltem quinque distizclis; Stilo semuper nullo; Calipters parva; Illalteres detereti

\section{FAr. XXI. XYLOPHAGIDE.}

AA. Antenna articulis plurimic sæe instructa, si raro sex tantum, tunc ralpi arliculis saltem tribus manifestis. X. Ala (nisi aborliva vel nulla) venis longitudinalibus saltem septem, (spuriis et decoloribus etiam consideralis): si raro sex tantum vel quinque manifestis, tune aiternos pilis verticillatis non instructæ.

7. Antennce capite transverso breviores, articulis longitudine latioribus, si vero aliquando in utroque sexu pauto 
longiores, non sátis Iongæ et alarum venæe longibit-r dinales sex vel quinque tantum, quarum tres rel quatuor posteriores omnino spurix.

\section{FAM. XXII. BIBIONIDE.}

ZZ. Antenne capite transverso satis longiores saltem in maribus, et si aliquando in utroque sexu parum langiores sunt capite, tunc articulis latitudine longioribus aut subglobulosis instructæ, vel alarum veuæ posteriores non spurix.

Y. Antennce piloso-verticillatie in utroque sexu nisi in mare plumatæ vel ramosæ, si raro subnudæe aut pubescentes in mare et fxmina, tunc alo (nisi abortivi vel nullæ) venis longitudinalibus distinctis saliem decem, et frons occllis non manifestis.

11. Antennce in mare plumatæ, et sì raro ut in fiemina verticillato pilosæ, tunc venis alarum posterioribns spuriis.

\section{Fam. XXIII. GIIRONOMIDA.}

W. W. Anternce nisi aliquando ramosæ in maribus, pilosoverticillatæ, vel tomentosæ, vel raro subnudæ in utroque sexu. - Alæ, (nisi abortivæ vel obliteratæ) venis longitudinalibus spuriis nullis vel subnullis.

2. Alo venis transversariis aliquibus tantum ron longe a basi, rel omnino nellis.

\section{FAM. XXIV. PHLABO'TONIDA.}

노․ Ala (nisi abortivæ rel nullæ) venis transversariis in medio ef non raro etiam in parte exteriori alarum prieditx. 
a. Als completæ, rarissimo abortivæ, sed numquam onmino obliterati.

\section{Fay. XXV. TIPULIDA.}

aa. Ala onnino obliteratæ.

\section{Fay. XXVI. CHIONEIDA.}

IY. Antennœ nudæ subnudæ vel breviter pilosæ, set pilis nou verticillatis manifeste. Alce venis longitudinalibus arl summum novem, sæpius octo, et sì raro venis decem præditæ vel abortivæ sunt, tunc ocelli manifesti.

b. Antennce arliculis duobus primis maxime incrassatis. Ocelli non manifesti.

\section{FAM. XXVII. ORPHNEPIILIDE.}

Antenna articulis duobus primis non aut parum incrassatis, et si aliquandio paulo crassis tunc ocelli distincti. ง. Alo: abortivæ, minimæ.

\section{FAIr. XXVIII. BERTEIDA.}

ce Ale latitudine ordituaria.

d. Fencora postica abdomine longiora. Tibia calcaribus indir slinctis.

\section{Fan. XXIX. ASTHENIDA.}

(") Il genere Sycorax Haliday: sarà forse il tipo di una famiglia In ermedia alle chironomidæe le Fhlæbolomidæ che si chiamerà Srcoraxide, e la sola stirpe contenente il genere doprà appellarsi Siconaxyr.s, che potrebbe forse anche esscre inclusa nelle chironomyda 
Ha Fenora postica atuomine breviora, ol si raro longita dine fere abdominis, tunc tibio calcaribus magis vel minus sed semper manifestis, ant saltem unico interiori.

c. Ala venis longitudinalibus marginem petentibus salten novem, el areola vera discoidali prallia.

\section{FAM. XXX. RIPHDE.}

ee. Ala venis longitudinalibus marginem pelentibus ad summum octo, areala discoidali vera dasti!ulie.

\section{FAM. XXXI. MYCETOPHILIDE.}

XX. Alce venis longiludinalibus quinque vel minus, et si rare sex lunc anetnnce pilis, verticillatis præditæ.

\section{Fam. XXXII. CECIDOMYDE.}

(Nota) Forse conreniva di far termiare questa serio di famiglie con quella del e Micetufilide, perchè fusse piu uaturale il passaggio dall' ordine dei Dilleri a quello delle Pulicarie che io gli fuccio seguire, essendochè le larve di alcuoi generi di quella famiglia ditterologica lilano un bozzulo entro del quale sulsiscono le oltime laro trasformazioni cosi :ome fanuo le larve delle Pulicarie.

Non ho adoltata aua tale disposizune perchè ma è sembrato che fosse pia regulare la serie di queste lamiglie secoudo l' ordine nel quale furous qui collocate; ma se ald alcuas piacesse pis di vedere le Micetofilide all' ultimo posto non avrebbe a far altro che cambiare la disposizione delle ultime laluiglie dopo le Berteide nel modo segunato Riphide, Asthenide, Cecidusyoe, Micetophilios, ed allora le stirpi. delle due ultime anderebbero cosi collocale.

$$
\text { Fak. CECIDOMIDE }\left\{\begin{array}{l}
\text { Stipes Lasioptroina } \\
\text { Micronyas }
\end{array}\right.
$$

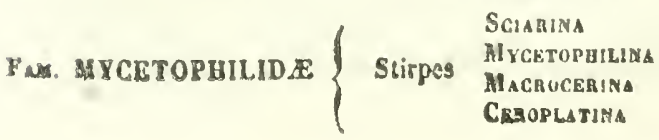




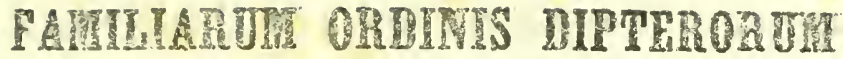

\section{STIRPES EUROPEE}

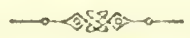

FAM. 1. OESTRIDA: Westw. Walk.

cestrlits Latr: Macq: - oestracunes Mreig: - Hearatomyza Fall: Zell: - aestrixa Rodn. - aestaidn Bigut.

A. Alarum vena omnes longitudinales in parte exteriori not cubitatx.

\section{Stirps 1. CESTRINA.}

AA. Alarum vence aliquæ longitudinales in parte exterior cubitatæ.

Stirps. II. HYPODERMINA.

Gă. 11. SYRPLiD R. Latr. Macq. West. Walk. syrpuicı Fall. Meig. Lòèw. Zett. - syaphis Latr. -

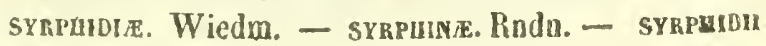
- Bigot.

A. Stilus antennarum dorsualis numquam perfecte apicalis \&. Scutellum tuberculis validis noo instractum. 
C. Arista antennarum omnino Ionge pilosa. Vena longituidinalis alarum quarta recta vel subrecta.

\section{Stirps III. VOLUCELLINA.}

CC. Arista antennarum nuda vel pubescens, at si aliquando distinctissime pilosa tunc vena quarta longitudinalis ante apicem intus valde sinuosa.

D. Vena quarta longiudinalis valde sinuosa, et sì raro minus, tunc femora postica apicem versus subtus dente valido instructa.

Stirps. IV. ERISTALINA.

DD. Kena quarta longitudinalis recta vel subrecta. Femoro postica apophisi apicali infera non distincta.

Stirps V. SYRPHINA.

BB. Scutellum tuberculis validis præditum.

Stirps VI. MICRODONINA.

AA. Stilus antennarum apicalis.

Stirps VII. CALLICERINA. 
FaMr. III. CONOPID E. Westw. Walk.

conopsaris Macq: Latr. Fall: Meig: Zett: - CoNopins. Rndu. - covopsidu. Bigot. - MYoparne Maeq. Zett. - occemide Ri-Des. - mYopiar Rndn. - MYopib. ligot.

A. Stilus antennarum apicalis.

Stirps. VIII. CONOPINA.

A. Stilus antennarun dorsualis.

B. Proboscis antice porrecta sed non bicubitata.

Stirps IX. ZODIONIXI.

BB. Proboscis bicubitata seu parte apicali retro flexa.

Stirns. X. MYOPIXI.

Fin. IV. MUSCIDE. Latr. West. Rndn. Walck. Maco. - Creophlla Latr. Maci. Westiv. - muscinf. Rum - calipterater R-Desv. - muscidn. Bigol.

A. Arista sen stilus antennarum nudus vel subundus vel breviter pubescens, sed pilis non distinclis, et lume cubitus vena quarta longitudinalis appendice spuria proditus, et abdomen segmento secunto setis rulibus ot erectis insiructo. 
Ti. Mroboscis longa exilis el bicubitata.

\section{Stirys XI. SIPHONINA.}

33. Proboscis brevis, et crassa, si raro exilis et elongdt tamen non bicubilata.

C. Epistomium vibrissis validis inslruclum, si raro parvis vel subnullis, tunc cutilus vence quintce longitudinaliz appendice venosa saltem spuria præeditus et capus thorace non latius.

\section{Stirps XII. TACHININ}

CC. Elyztomium vet mutum bel selis exilibus, seu pilis parvis instructum, et si raro vibrissa valida adsunt, uns caput thorace latius, et cubilus vence quinte longitudinalis non appendiculatus.

\section{Stirps XIII. PHASIIYA.}

4. Arisla sen stilus antennarum plunalus ant distinete pilasus, si vero pubescens tanium, lunc cubilus vente quinto longitudinalis non appendiculatus, vel abdomer. segmento secundo sibnulo aut pitis exilibus flexis el non selis erectis prædito.

10. Arista superne el inferne snb-argue pilosa, si vero aliquando inferne pilis paucis vei brevioribus instructa, tunc proboscis brevis el membianosa.

w. Abdominis dorsum in segmento secundo el non raro etiam in primo selis aliqubus validis erectis at distinctis proditum: si vero aliggnando macrocheta erecta io duobus prixois segmentis nou adsunt, tupe ewhims 
veme quinte longiludinalis appendice saltem spuriat manifesta.

\section{Stirps XIV. DEXINA.}

EE Abdominis segmenta dıo prima macrochetis ereclis destituta: ad summum pili majores aliqui erecti adsunt. Cubitus venæe quintoe longitudiualis appendice nulla neque spuria præditus.

Stirps. XV. MUSCINA.

DD. Arisla vel superne tantum distincte pilosa vel inferne pilis aliquibus et minoribus instructa, et tunc proboscis cornea et porrecta.

Stirps XVI. STOMOXIDINA.

\section{FaM. V. ANTHOMYDA Rndn.}

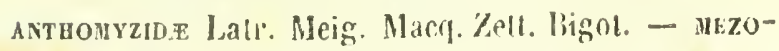
MID.E R-Desv.

A. Tibice antica el postice extrinsecus sclula crecta prope apicem instructæ præter alias si adsunt. - Vena secunda longitudinalis superne ciliis brevibus non pectinata.

B Episcomium setis validis sältem duabus, et sxpius eliam aliis minoribus instructum. 
C. Calipterorum squamæ inferæ a superis non omnino tectæ, et si raro sub-æquales, tunc oculi maris contigui atit subcontigui allt saltem a medio frontis usque ad radicem antennarum manifeste divergentes.

\section{Stips XVH. ANTHOMYNA.}

CC. Calipterorum squamæ inferæ a superis omnino tectæ. Oculi maris vel distantes in fronte fere ut in fæmina, vel usque ad radicem aniennarum sub-paralleli.

\section{Stirps XVIII. SCATOPHAGINA.}

BB. Evistomiun setis omnino destitutum.

\section{Stirps XIX. SCYOMYZINA.}

AA. Tibiæ anticæ rel postica rel omnes seta nulla erecta sub-apicali, et si raro adest, tunc vena secuuda longitudinalis superne ciliis brevibus pectinata.

D. Venu secunda longitudinalis superne pectinata. Stirps XX. ORTALIDINA.

DD. Vena secunda longitudinalis superne non pectinata.

E. Vence lonsitudinales tertia et quarta, vel quarta et quiuta versus apicem manifeste convergentes, et si minus distincte convergentes, tunc epistomium nudum, et femora antica setulis subtus non ciliata. Stirps XXI. TANIPEZINA.

EE. Vence longitudinales tertia et quarta, ut quarta et quinta versus apicem non convergentes, aut divergentes, et si raro paulo approximatæ aut illæ aut istæe, tune 
epistomium setulis vel pilis instructum, et simul femora antica subtus splulis ciliata.

Stirps XXII. LONCHEINA.

Farf. VI. AGROMYZIDE Rndn.

acaliptera Meig. Macq. Westw.

\$. Vence transversariœ alaruin duæ, magis vel minus distan-" tes a basi; infera magis distante.

B. Areola anales et basilares completæ et distinetæ, et si raro vel unæ vel alleræ vel omnes incompletæ vel indistinctæ sunt, tunc tarsi postici ad basim dilatatoincrassati.

C. Vena prima longitudinalis basi duplex apice simp!ex. Stirps XXIII. AGROMYZINA.

CC. Vena prima longitudinalis simplex a busi ad apicem.

D. Epistomium setulis et pilis crassiusculis destitutum. Stirps XXIV. CHiLIZINA.

DD. Epistomium setulis vel pilis crassiasculis præditum. Stirps XXV. COPROMYZINA.

BB. Areolo basilares et sxpe eliam anales rel incompletxe vel indistinctæ, - tarsi postici nunquam dilatato- incrassati. 
i. Arista nuda re! subnuda, si raro pubescens, tunc pubescentia superne et inferne acue ionga et tibiis setula nulla erecta sub-apicali. - Epistomium selulis inferis pendentibis numquain marginatum.

F. Epistomium setulis et pilis crassiuculis destitutum et s! raro pilis rel setis aliquibus instructum, tunc vena costalis cuntra apicem tertiæ longitudinalis sistens, et quarta longitudinalis perfecta.

\section{Stirps. XXVI. CHLOROPINA.}

1F. Epistomium setulis ve! pilis validiuseulis instructum. Vena costalis contra apicem quartæ longitudinalis producta, ei si raro contra apicem tertiæ sistens, tunc quarta spuria et subinlistincta.

\section{Stirps XXVII. OSCININA.}

EE. Arista distincte pilosa vel radiala saltem superne, et si raro subnuda vel suhaque pilosula seu pubescens superne el inferne, turc tibice aliquze setula erecta sub-apicali instructa, vel epistomium inferne setulis pendentibus marginatum.

G. Arista superne tantum ciliata vel radiata, el si pilosula. vel pubescens, pubescenlia supera iongiore; vel si nuda aut fere nuda, tunc epistomium setulis inferis pendentibus marginatum, tibiisque posticis et intermediis setula nulla erecta sub-apicali.

\section{Stirps XXVIII. EPHIDRINA.}

GG. Arista ciliis, pilis, vel pubescentia superne et inferne sub-reque longis, et si aliquando subnuda, tunc episto- 
minar setulis pendentibus non marginatum, et tibiis posticis vel intermediis setula erecta sub-apicali distincta. Stirps XXIX. DROSOPHILINA.

AA. Venœ transversarice nullæ, vel unica tantum ad basim alarum retracta, si vero duæ adsunt, tunc supera magis distans a basi.

Stirps XXX. ASTEINA.

TAM. VII. PHORIDE. Walk.

hypocera Latr. Macq. Westw - trineviddes Fall. Zett. - trineure Meig. - puytomyzides (Partim) Fall. - PHORIN R Rndn. - uYPOCERIDE Biget.

A. Arista distincte pilosa. - Frons satis Producta. Stirps XXXI. ME'TOPININA.

A. Arista subunda - Frons non distincte producta. Stirps. XXXII. PHORINA. 
28

FAM. VII. LONCOPTERIDA. Walk.

oxiptera Meig. - piytomyzine (partim) Fall. Zett.

- congoptering Macq. Rndn. - senopinida Westw.

- loncopterindit. Bigot.

Unica Stirps. XXXIII. LONCOPTERINA

FAm. IX. PLATYPEZID A. Walk.

pratypezine Fall. Meig. Macq. Zelt. - dolicopodea

Latr. - callonine Rndn. - senopinida Westw. pLATYPEZINIDU. Bigot.

A. Atce renula transversaria unica in parte basali, inde: areola nulla intermedia.

Stirps. XXXIV. OPETINA.

A. Ale venulis transversariis aliquibus, quarum una in parte exteriori sita.

Stirps XXXV. PLATYPEZINA. 
Fam. X. PIPUNCULIDÆ. Walk.

cepialupsida Latr. Macq. - anegacepdala Meig. pIPUNCULINe Rndn. - pIPUNCULINI Zelt. - SENOPINIDE Westw.

Stirps Unica XXXVI. PIPUNCULINA.

FAM. XI. DOLICOPIDE. Westw. Walk. dolicopodu Wiedm. Bigot. - dolicopodes Latr. Fall. Meig. Zetı. - Doulcopoda Macq. - dolicopins Rudn.

A. Stilus antennarum dorsualis, raro subapicalis. Stirps XXXVII. DOLICOPINA.

A . Stilus antennarum apicalis.

Stirps. XXXVIII. RAPHHAA. 
30

\section{FAM. XII. EMPIDE. Rndn.}

eMpides Latr. Macq. - earpdia Fall. Meig. Loèw.

- taGhidnomyde Mgn. - hybotrde Meig. Latr. Zett.

- tachydronydes. Wiedm. - empidine Rndn. EMPIDIr. Bigot.

A. Venae longitudinales alarum ad summum quinque, excepta: ultima postica, nunc distincta nunc vix aut non manifesta. Stirps XXXIX. TACHIDROMYiva.

AA. V'cna longitudinales alarnm sex vel septem, ultima postica excepta, nunc distincta nunc parum aut non manifesta.

B. Pedes antici raptorii, scilicet coxis valde elongatis femoribusque simul incrassatis.

\section{Stirps XXXX. PHYLLOdROMINA.}

BB. Pedes antici non raptori, ideo etiamsi femora incrassata coxce tamen haud valde elongatæ.

C. Proboscis perpendicularis vel retro flexa, numquam horizontalis nec surstm erecta.

Stirps XXXXI. EMPIDINA.

CC. Proboscis vel horizontalis vel sursum erecta.

Stirps. XXXXII. HYBOTIDINA. 
FA.Y. XII. LEP'TIDE. Westw. Walk.

Leptides Latr. Meig. Macq. Loew. xylotomyde Mgn

Macq. - nydasil. Latr. - anthracide Fall. - ragionides. Latr. - LePTidine Rutu. - thereviN.

Kndn. - Leptidi. Bigot. - вомвyLidi. Bigot.

A. Antennce arista vel stilo distincto, numquam loco stili articuli aliqui adsunt parum distincti.

3. Arista longa manifeste apicalis vel apparenter dorsualis (In generibus Atherix el Ibisia).

Stirps XXXXIII. LEPTIDINA.

BB. Stilus satis brevis apicalis.

C. Palpi longi et lati. - Proboscis pendens sursum non erecta. Stirps XXXXIV. SPANINA.

CC. Palpi breves at non dilatati. - Proboscis contra faciem elevata.

Stirps XXXXV. THEREVINA.

1A. An'ennec stilo et arista nullis, loco stili articuli duo adsunt pirtum distincti.

Stirps XXXXYI. PACHISTOMINA. 
Fam. XIV. ASILIDE. Westw. Walk. Asiucr. Latr. Fall. Meigr. Macq. Zelt. Loew - asmin Rodn. - asilivi Bigot. - asiluca Loew.

A. Vence longiludinales secunda et tertia sejunctim costalm attigentes.

Stirps XXXXVII. DASIPOGONINA.

AA. Vence longitudinales secunda et tertia conjunciæ aste costalem.

B. Antenna stilo vel arista præditæ.

Stirps XXXXVIII. ASILINA.

BB. Antennce stilo el arista destitute.

Stirps XXXXIX. LAPHRIINA.

FAM. XV. MYDASID E. Westw. Bigot. Midasil Latr. Macq. - Midisise Rndn.

Unica Stirps L. MYDAS̈INA. 
Fast. XVI. BOMBYLIDE. Westw. Waill.

bonbylanal. Meigr. Wieda. Macq. Zeal. Loew. Bonbyline Rndn. - eonbyldon. Bigol. - nemestei mide Macq. - Nemestrinine Riudu. - anthraci. Lall Macq. - anturaciues. Fall. Zelt. - anthascine hodn

A. Ala nisi reticulatæ venis longitudinalibus in parte apicali salten quatuor paralleliter decurrentibus.

Stirps LI. FALLENINA.

AA. Alæe non reticulatæ, et venis longitudinalibus ad summum duabus paralleliter ad apicem deccurentibus.

Stirps. LII. BOMBYLINA.

\section{FaM. XVII. CYRTIDA. Rndn.}

vesciculosa Latr. Meig. Macq. - ixplata Mgn. Stratyomyde Fall. - acroceride Westw. Walk. acrocerine Zett. - ogcodise Rndn. - vescigulosid.e Bigot.

A. Proboscis distincte elongata.

Stips LIII. CYRTINA.

A. Proboseis brevissima. 
B. Antenna stilo vel arista distinctis.

Stirps LIV. HENOPIDINA.

BB. Anternœ stilo et arista destitutæ.

Stirps LV. ASTOMELLINA.

\section{FAN. XVIII. SCENOPINID\& Rondn. Bigot.}

scenopidi Mgn. Fall. Macq. Zett. - stratioryda. Latr. - SCENOPININ Rndn. -

\section{Unica Stirps LVI. SCENOPININA.}

Far. XIX. STRATIOMYDE Macq. Westw. Zell, Bigot. Walk. Lò̀w.

stationydes. Latr. Fabr. Meig. Hacq. - stratioMYDINE Rndn.

A. Scutellum nec dentatum nec tuberculatum. Stirps LVII. CHRYSOMYNA. 
IA. Scutellum vel dentatum vel tuberchlatum.

Stirps LVII ODONTHOHI NA.

Fan. XX. TABANIDAE. Westw' Walk. Bigot.

тавами. Latr. Meig. Macq. Zelt. Loèiv. - тввамixt. Rndn.

A. Antenne articulo tertio sulciolis circularibus ät suntmum quatuor - Proboscis breviuscula et crassa.

Stirps LIX. TABANINA.

A. Antennoe articulo ultime sulciolis circularihus sex. Proboscis exilis et elongata.

Stirps LX. PANGONINA.

FAM. LXI. XYLOPHAGIDE Bigot. Walk.

xycopmagir. Fall. Meig. Latr. Macq. Zett. Lòèw. cOENoryde Westw. - STRatiomydiñ (partim) Rndn. - sicarir. Latr. Macq. - beride Westw.

A. Tibice aliqux nisi omnes apice spinis validis praditx. 
B. Scutellum iuberculatum.

Slirps LX!. COENOMYNA.

BB. Scutellum non tuberculatum,

$$
\text { Slirps LXII. XYLOPHAGINA. }
$$

A. Tibiæ omnes spinulis apicalibus destilulæ.

Slirps LXVIII. BERIDINA.

FAr. XXII. BIBIONIDE Macq: Bigot. Walk.

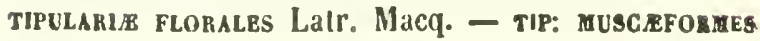
Mgn. - tip: latipennes Mgn. - hirteides Zelt. scatopsides. Zelt. - bibionides Macq: Westw. BIBIONINE Rndn. - sIMULiN.x. Rndn.

A. Areola dux basilares alarum distincia.

Stirps LXIV. BIBIONINA.

A. Areola basilaris unica, vel unlla.

B. Ocelli distincti. - Vena posticæ alarum tres vel quatuo: spuriæ.

Stirps LXV. SCATOPSINA.

BB. Ocelli non manifesti. - Vence posticæ alarum plures spuriæ.

Stirps LXVI. SIMULINA. 


\section{Fam. XXIII. CHIRONOMIDE Walk.}

ccroides Lallr. Marr. Zell. - culicine Zelt. Mindri.

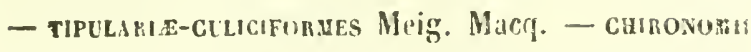
Zelt. - culcid.s Wesiw. Rndn. Bigot. - chroonomdes Westw. Mueq. chronomine lindn.

A. Proboscis brevissina.

b. Vence longitudinales septem tantum, et si plures fere omnes spuria. - l'edes antici saltis longe a sequenlibus orientes.

\section{Stirps LXVII. CHIRONOMINA.}

BB. Venue longitudinales octo et ultra, fere omnes non spuriæ. - l'edes omnes ad originem sub-æque distante. Stirps LXVIII. CORETHRINA.

A. Proboscis satis elongala.

Stirps LXIX. CULiCINA.

Fam. XXIV. PHLOEBOTOMIDAE Rndn Lòew. Walk. psicodides Zett. - tiplle-xoctueformes Mgn. Gallicole (partim) Macq: - phalenoto.s Macq. Bigot. - pualenoides Macq. - psicodin.e Rindn.

A. Antennce filiformes non moniliformes. - Palpi articulo ultimo satis longiore vel longissimo.

Stirps LXX. PHLGEBOTOMINA. 
A. Antennice moniliformes. - Palpi articulo ultimo precedentibns sub-aquale.

\section{Stirps LXXI. PSYCODINA.}

Fа. XXV. TIPULIDA Macq. Rndn. Bigot Walh. tipulanie-terricole Latr. Macq. Stœger. Lòdw. tipulides Westw. Zell. - tipun: rostrate Mgn. TIPCLNE Rndn.

A. Palpi articulo ultimo forma et longitudine parum diverso a præcedentibus.

Stirps LXXII. LIMNOBIINA.

AA. Palpi articulo ultimo satis longiore præcedentibus, exiliore et tlexili.

Stirps LXXIII. TIPULINA.

\section{FAs. XXVI. CHIONEID $\approx$ Rndn. Bigol.}

caroneides Zett. - tipur: trRricole Macq. - culoNEINA Rndn.

Unica Stirps LXXIV. CHIONEINA. 
FAM. IXVII. ORPHNEPHILIDE Rnda.

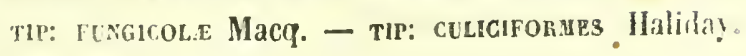

Unica Stirps LXXY. ORPHNEPHILINA.

FAM. XXYII. BERTEIDE Rndn.

Unica Stirps LXXYl. BERTEINA

FAM. XXIX. ASTENIDE Rndn.

Unica Stirps LXXVII. ASTENINA.

FAM. XXX. RHYPHIDE Macq. Walk. p.IP世u Zett.

Unica Stirps LXXVIII. RHYPHINA. 
FAM. XXXI. MYCETOPHILIDE Macq. Bigot. Walk. Tip; FUngichle Latr. Macq. - Tip: Fungivora Meig. - rycetupHLin.e Zell. - nycetopulides Westw. schopusun.e lindn.

A. Areola basilaris alarum postice a vena septima longitudinali circuul crivla.

B. Antennce capite et llorace conjunctim breviores, articulis subquadratis, et sape longiludine latioribus.

Stirps LXXIX. CEROPLATINA.

18. Antenna capile et thorace conjunctim longiores, nisi longitudine corporis toli et ultra, articulis cylindricis sæpius latiludine longiures.

\section{Slirpr' LXXX. MACROCERINA.}

A. Areola basilaris postice a vena Iongibudinali tertia, vel a quarta sed numquam a septima circumscripta.

C. Vence longitudinales alarum septem margini productix, si vero numero minori, lunc lilice quatuor postica extrinsecus spinis validis serratæ.

\section{Stirp LXXXI. SCIOPHILINA.}

C.C. Vence longitudinales alarum sex margini productæ. Tibice postica extrinsecus non spinulusæ.

Stirps LXXXII. SCIARINA. 
4а. XXXII. CECIDOMY DE Macr. Bigot.

cecidonyzides Zett. - cecidomydes Westw. - ripur: gallicol.s Latr. hiogn. Macq. - Gecidonyae Rndn. cecidoryzide Walk. - Lestremine Rodn.

A. Tarsi articulo basali longitudine ordinaria. - Ocelli adsunt. - Vence longitudinales sex vel quinque a costali distincta sed aliquando intermedia aliqua spuria et vix distinguenda ennumerata.

Stirps LXXXII. MICROMINA.

A. Tarsi articulo basali brevissimo. - Ocelli non distinguendi. - Vena longitudinales a costali distincta ad summum quatuor spuriis etiam consideratis.

Stirps LXXXIV. CECIDOHYYNA. 


\title{
DIPTCRORUM GENERA
}

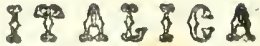 \\ HN ORDINEMI DISPOSITA:
}

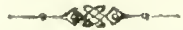

STIRP I. CSTRINA Rndn.

Srmomara Familiz OEstriảortan.

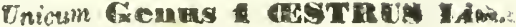

Snecies Typica aesraus RQur. Clark.

Stins II. HIPODERMiNA Rndn.

Sronur: Familix OEstridortum.

A. Fence longitudinales alarum quarta ei quinta rejunctim. costalem attingentes.

S. Pena longitudinalis quarta non angulatim cubitata nes appendiculata.

Cenus frypoderma Clark.

Species typ. astrus Bovis Fabr. 
17. Fend quar'd longitulinalis angulo distinctissima cubitata, cubito appendiculato.

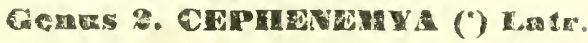

Spec: Typ: astrus Trompe Fabr.

1.A. Venoe longitudinales alarum quaria et quinta conjunetat antequam costalem altingant.

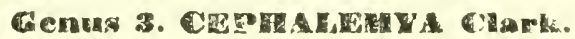

Species: Typ: aestrus ovis Lin.

Irmes lil. VOlucellina Rndn.

Syмомат: Familize Syrphidoruan.

A. Vene longitudinales secunda et terlia sejunctim costalem atligentes.

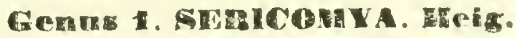

Species Typ: Syapitus Bonbyliforals. Fall.

(1) A questa famiglia appartiene enche it genere Hrodemagena formato per una specie che trovasi solamente nei paesi piu Setrentri- nali d' europa, ma non lo credo tanto distinto dal genere Fypoder*a per meritare di restarne disgiunto.

Di questo genere si trovano specie nella Lapponia in Norregia in sessovia ad in Austria e non ancora in altri paesi meno boresti. 
AA. Vence longitudinales secunda et tertia conjuncta ante costalem.

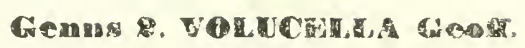

Spec. Typ. Musca lnivis Lir.

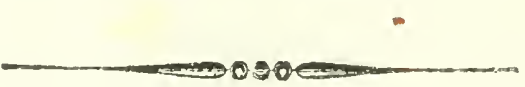

STIRPS IY. ERISTALINA Rain.

Srnowis: Famil: Syrphidortm.

A. Femora postica apophisi valida apicali non instumctio

B. Antennarum articulus tertius longitudine latior.

C. Femora postica valida sed non distincte incrassata.

Oculi in utroque sexu magis vel minus distantes.

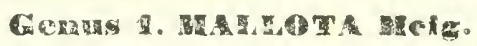

Species Typ. Ertstalus Fucteoruts Fathr.

CC. Femora postica valde incrassata. - Oculi maris contigui vel subcontigui.

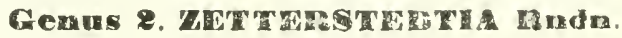

Species Typ. Syrpus Cymriciforaus Vall.

BB Antennarum articulus tertius vel subeovatus vel slbcircularis, non longitudine latior. 
D. Antenna articulo secundo longitudine tertii in utroque sexu. - Arista maris apice dilatala.

\section{Genug 3, PLATyNocheres Wed.}

Spec. Typ. Eristalus Setosus Fabr.

ID. Antenne articulo secundo longitudine minori tertii in utroque sexu. - Arista in mare nóndilatata ad apicem.

E. Vence longitudinales secunda el tertia conjunctæ ante costalem

\section{Cenus 4. Enstals Fabr.}

Species Typ. Musca Tenax Lin.

EE Vence longitudinales secunda el tertia sejunctim costalem alligentes.

F. Femora postica haud distincte incrassata. $=$ Ocwli maris contigui in fronte.

6. Antennarum articulus tertius subciıcularis - Pedes validi.

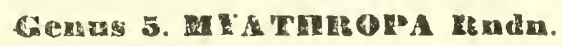

Spec. Typ. Musca Florea Lin.

GG. Antennarum articulas tertius sub-ovalus. - Pedes graciles.

\section{Gerus 6. HDE Rreq.}

Spec. Typ. Dibea Easciata Macq.

FF. Femora postica distincte incrassata. - Oculi in utroque sexu wagis vel minus distantes in fronte.

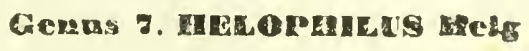

Spec. Typ. Mesea Pexdurs Lis. 
AA. Femora postica distinclissima apophisi apirali subtas instructa.

H. Oculi pilosi in utroque sexu, - Facies non carinata.

Cenus \%. Bestobov. Lar.

Species Typ. Merodon Cuavipes Latr.

HH, Oculi nudi. - Facies carinaia.

Gemus 8. Tromption

Spec. Typ. Tropidia Fasciata Meig.

Suadi V. SYRPHina Fnd.

Sraonn: familia Syrphidorum.

A. Temora postica inferne vel denticulo aliquo instracia vel seriatim spinulosa vel serrata, el si inermia, tunc wsula prima transvet'saria magis distans a basi quam ab apice areolo retro-pnsilx.

B. Femora postica nec inferne serrata nec serialim spinulisa, aliquando denticulo aliquo instructa.

C. Vene lorgitudinales secunda el teria apice conjuncta anterguan costalem attingant.

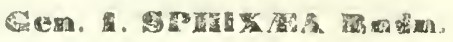

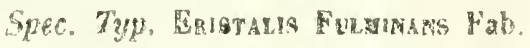


BC. Vence longitudinales secunda et tertia sejunctim cosinlem attingentes.

D. Facies in medio non tuberculata nec inferne distiact, producta. - Articulus tertizs anternarum subcireu. laris numquam Iongiludine latior.

I. Frons non dislincle producla. - Areolarum exteriorum ultima postica appendice longa apicali oblique marfricem postcriorem alarum allingente.

Genas 2. MEg

Spec: Typ: Rlusca Droputhalua Lin.

EE. Frons distincte producta. - Arcola ultima postica exteriorum, appendice apicali brevi $\epsilon \hat{\imath}$ recte margia! postico alarisn producta.

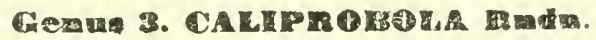

Spec: Typ: Mulesia Speciosa Fabr.

01. Focies in medio toberculata et inferne distincie producta. - Anfenarum articnlus tertins magis rel minus distincte longitudine latios.

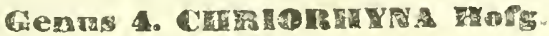

\section{Spec: Typ: Millzsia Feocansa Heig.}

38. Femora postica inferne serrata vel spinulosa, saltem ia parte apicali.

F. Finula prima transversaria magis distans a basi quarn ab apice areolce retro-posita.

fr. Sutillum margine non granuloso. - Facies distingte excarata. 
48

II. Articulus tertius antennarum ovalus. Arista longe a bas articuli tertii inserta. - Oculi maris etiamsi proximi non contigui.

\section{Genus 5. IB Is ChIPALPS maeq.}

Spec: Typ: Xrlota Valga Meig. .

HH. Antennarum articulus te"lius circularis. - Arista prope basim arliculi tertii inserta. - Oculi maris iu fronte contigui.

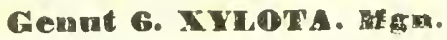

Spec: Typ: Musca Secins Lin.

GG. Scutellum margine granuloso vel scrvulato. - liveies omnino plana.

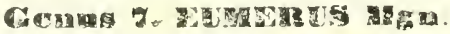

Spec: Typ: Pipiza Strigata Fall.

FF. Venula prima transversaria magis proxima busi quăa apici areolat retro positæ.

I. Abdomen prope basim non manifeste coarctatum.

K. Vena longitudinales quarta et quinta ad apicem cunfusie et simul margini produclæ - Facies maris gुbbosx.

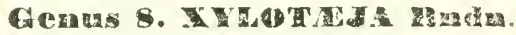

Spec: Typ: Mllesta Vara Fabr.

KK. Fena quinta longitudinalis quartæ conjuncla satis longe a costali - Facies in utroque sexu non gibbosa sed carinata.

Genus 9. Symera s. verv.

Spec: Typ: Musca Piplens Lin. 
11. Abdomer segimentis prope basin distincte coarctatis.

L. Antennarum articulus tertius subovato-elongatus Vena quinta longitudinalis angulatim flexa.

\section{Genas 10. ASCIA threl.}

Spec: Typ: Merodon Podagricus Fabr.

4.t. Articulus terlius antennarum subcircularis, non elongalius - Vena quinla longiludinalis arctratim flexa.

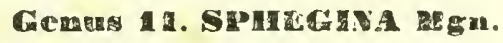

Spec: Typ: Mitrsia Cluniprs Fall.

AA. Femora inermia - Venula prima transversaria magis proxima basi quam apici areola retro posita.

M. Antenne brevissimæ, et sì raro paulo elongatie lunc pendentes vel flexæ, sen non horizontaliter erectæ.

N. Abdomen segmentis prope basim distincte coarctatis.

0. Fena quarla longiludinalis oriens circiter contra secumdam transversariam.

\section{Gearus 12. MACA wahr.}

Spec: Typ: Bacca Elongata Fabr.

00. Tena quarta Longitudinalis oriens satis ante cemulan secundam transversariam.

I. Arista antennarum pilosula - Abdomen segmento secundo etiamsi angustato, depresso.

Cenus 13. Sprugastrat Aadm.

Sype: Typ: Spazigster Apesivisi Rndu. 
BP. Arista antennarum nuda - Abdomen segmento secundo cylindrico.

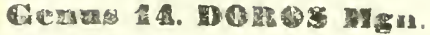

Spce: Typ: Milesia Conopsea Fubr.

Niv. Abdomen segmentis prope basim uon distincle coarclatis.

(1. Racies in medio tuberculo elevato vel gibbosa in utroque sexu - Antenno arliculo tertio subcirculari vel breviter ovato - lrons faminæ non Iransversim sulcio-

làia nec seriatim punclulala.

7. Vena longitudinales prima, secunda et terlia in costali apicibus subæque distantibus - Organa copulatoria maris inflata, genilali penicillato.

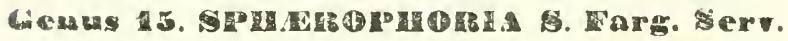

Spco: Typ: Musca Scripta Lin.

hih. Fence tres prime lougitudinales, in costali apicibus varic distantibus - Organa copulatoria maris non inflata, et si paulo producta vel crassa, genitali non penicillato.

S. Arista antennarmm brevis, manifeste triaticulata.

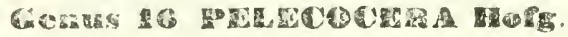

Spec: Typ: Pelecocena Tricireta Hofg.

S5. Arista elongata, articulo unico distincto.

T. Fena spuria ordinaria non interrujta el ubique distincta - Arista nuda.

1. Fen prima transwersaria magis proxima basi quaco apici 
areola retro positæ - Anterma articulo tertic orato 7. aculi omnino nudi.

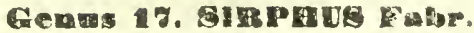

Spec: Typ: Musea Ribesu Lin.

iY. Deuli hirli vel tomentosi.

Cำง

Spec: Typ; Musca Pyastral Lin.

10. Venaprima transversaria sub-aque distans a basi ct apice arcolce retro positæ. - Antenna articulo tertio circulari.

Gents 18. CHITYSTHARS Enda.

(Genus Ferdinandæa Fndn Olim.)

Spec: Typ: Conops Curreus Scop.

'T"T. Y'na spuria ordinaria aliqna parte interrupla vel ubique parum aut fere nihil distinguenda - Arista tounentosa vel breviter pilosula.

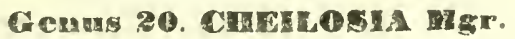

Spec: Typ: Enistalis Scutellata Fabr.

1Q. Lacies non tuberculata vel in utroque sexu vel saltem in femina; qando facies maris tuberculo pradita, tunc frons famina transversim sulciolata vel punctulata: si vero in utroque sexu in medio paulo elevata vel stoscarinata, tunc antennarum articulus tertius distincte clongatus, seu nec subcircularis nec breviler oralus. 
32

\. Oculi ominino nudi.

3. Angulus apicalis inter venas qlartam el quintam recins vel obtusus nunquam manifeste acutus.

1. Antennarum articulus tertius elongalus. seu nec circuliris nec breviter oratus.

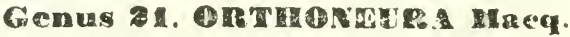

Spec: Typ: Erictilis Nobius Fall.

YY. Antennœ articulo tertio circulari vel bieviter ovato. \& Antennce articulo tertio breviter ovato. - Vena tertic longitudinalis costalem attingens satis extla apicem quintQR, ubi conjungilur quarto.

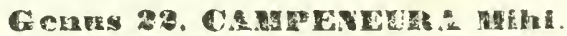

$$
\text { Spec: Typ: (Nova) C. Venusta Mihi. }
$$

2. Antennce articulo tertio circulari - Vena tertia longitudinalis costalem atlingens contra apicem quinse ubi conjungitur quarece.

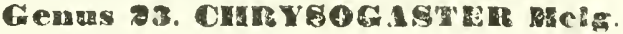

Spec: Typ: Cyrysogaster Splendens Mgl.

ZZ. Angulus apicalis inter venas longindinales quartam et quintam magis vel minus acutus.

b. Epistomium non rosiriforme etiamsi paulo productun.

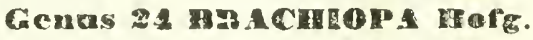

Spec: Typ: Ruyngu Bicolon Fall.

4olv. Ipistomium value productum, rostriforme.

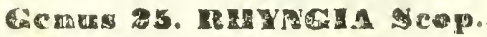

Spee: Typ. Rayeg Rostrata Scop. 
XX. Oculi pilosi vel sallem tomentosí.

If Antenne articulo tertio subcirculari, vel breviter ovato.

c. Vena longltudinalis quinta a cubito ad apicem recta. Abdomen non aut vix longior thorace.

Genrs eb. PSiLOT an.

Spec: Typ. Psilota Anturagina Mgn.

ce. Vena quinta longitndinalis supra cubilum magis vel mints intus llexa. - Abdomen circiter longitudine dinpla Thoracis (*).

\section{Gemus 2\%. Pariza rall.}

Spec: Typ: Mrsca Noctiucs Lin.

hils. Anennee articulo tertio distincte elorgato, seilicet nec çirculari nec hreviler ovato.

4. Vena prima longitudinalis producta ultra apicem areolic basilaris anticx. - Vena quinta conjuncta quarta ultra apicem terlice.

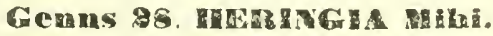

$$
\text { Spec: Typ: }\left\{\begin{array}{l}
\text { Heringia Zetterstedtii Rudu. } \\
\text { Pipiza Hering Zelt. }
\end{array}\right.
$$

ada. Vena prima longitudinalis non producta ultra apicetn areola basilaris anterioris. - Vena quinta conjuneta quartee contra apicem tertice.

"Il genere Trigliphus del Lòw andrà collocato prima delle Pipiż se si roglia diviso dalle medesime, e sarebbe distinto per la larghexa -degli annelli addominati 2 e 3 che occupano quasi tutta la parte dorsale. 
e. Oculi distincte et longe pilosi.

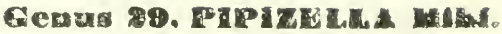

Spec: Typ. Pipiza Virens Fabr.

ce. Oculi iasciato-tomentosi.

\section{Cenus 30. PATAGoA Latr.}

Spec: Typ: Paragus Bicolor Latr.

MM. Antennce capite longiores et horizontaliter erectse.

. Arista in medietate apicali arliculi tertii antennarum inserta.

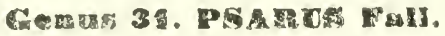

Spec: Typ: Psarus AbDorivalis Fabr.

r. Lrisia antennarum prope basim articuli tertii inserta.

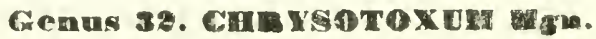

spec: Typ: Musaa Arcuata Lin.

Swas Y!. MICRODONINA Rniln.

Srwonus: familia Syrpidorum.

Unioum fienas 1. AmHabris Latr.

Spec: Typ: Musca Mutabilos Lin. 
Srikrs VII. CALLICERINA Rnda.

Cerintr Bigot.

1. Versa quarta longitudinalis subrecta et intus nor apperdiculata.

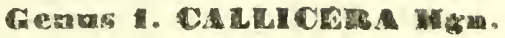

Spec: Typ: Syrpies Auratus Rossi

A. Vena quarta longitudinalis intus distinctissime incurvals. et appendiculata.

5. Frons in petiolum antenniferum valde producia. domen segmento secundo haud distincte, in medis coarctalo.

\section{Gerus 8. Cinta vab.}

Spec: Typ: Musca Conopsoides Lin.

BB. Frons haud distincle producta. - Abdomen segments secundo in medio satis coarctato.

Genus 3. SPHYXIMOR PIN nadin.

Spec. Typ. Ceru Subsessiurs Meig. 
56

Erikes VIII. CONOPINA Rndn.

(partin) Srionm: Famil. Conopidorum seu cosorskriz Mact. Latr. Fall. Meig. Zett. - conopine Rndn. - conopsidn Bigot.

A. Femora postica si crassa regulariter incrassata. - Aldominis segmentum secundum haud distincte coarctatum.

i. Proboscis brevissima et ciassiuscula, non aut vix epistomium superans.

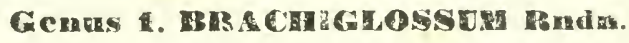

Spec: Typ: Leopoldius (olim) Rndn. Erosrriruy Rndn.

BD. l'roboscis exilis et ultra epistomium satis aut valde producti.

1). Lemora postica ut anteriora non, aut parum incrassata, et longa circiter ut tria prima segmenta abdominis. - Theca ventralis fominæ porrecta et rotundata.

Genus 3. GPHXXSOMA Meni.

Spec: Typ: Conops Quadrifasciatus De-geer.

D). Femora incrassata, posticis satis crassioribus et vix longis ut duo prima segmenta abdominis. - Theca ventralis focminæ indistincta.

Gemus 3. CONOPILLA Hndro.

Spec: Typ: Conops Cerifformis Mgrl.

A. Femora postica in parte basali tantum irregulariter 
incrassata. - Abdomen segmento secundo maxime coarctato.

Gears 5. Covar

Spec: Typ: Conops VitTata Fabr.

Stinps IX. ZODIONINA Rndn.

hyoparie Macq. Zelt. - occenyoe R-Desv. - myoPINE Rndn. - Myopide Macq. Bigot.

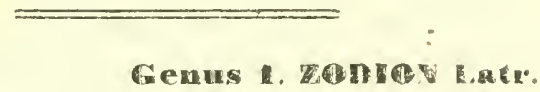

Spec: Typ: Zodion Cinereun Hgn.

Stirps X. MYOPINA. Rndn.

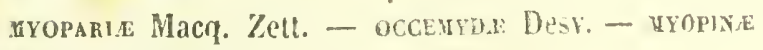
Maç. Rnd. Bigot.

A. Ar eola analis inferne in angulum aculum satis producta ultra apicem areola postica basilaris. - Abdomen tominæ oviducto non ensiformi, maris appendicibus filiformibus destitutum. 
B. Antenna distincte longiores, saltern tepplo, distantia a earum radice ad oculos. - Oculi a latere duplo circiter longiores periscomio sub-oculari. - Theca ventralis fœmina producta et aciminata.

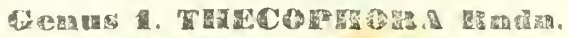

\section{Spec: Typ: Myopa Atra Fabr.}

BR. Antenna longæ circiter ut distantia oculoruan ab apice frontis, numquam bilongiores earum distantia $a b$ oculis. - Theca ventralis in foemina non distuncte producta, et si panlo porrecta, tunc oculi a latere vix longi ut perislomium sub-oculare.

C. Antennarwm articulus sectundus ad summum longiludine tertii sel minori in utroque sexu.

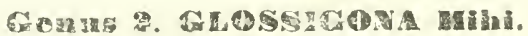

\section{Spec: Typ: Mropa Ogculta Wiedm.}

CC Antemarum articulus secundus circiter bilongior tertio, vel paulo minus.

B. Abdomen segrmentis secunlo et tertio longiludine subæqualibus. - Oculi a latere, breviones peristomio sub-ocularis.

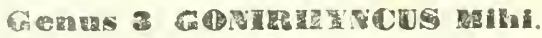

Spec: Typ: (Nova) G. Dispar Mihi.

DD. Alduminis segmentum secundum circiter bilougius tertio. - Oculi a lateie longi circiter ut peristomium snb-oculure rel longiores,

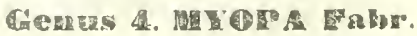

Spec: Typ: Conops Ferruarneus Lin. 
AA. Areola analis brevis ut basilanis postica, et angulo apicali posteriori subrecto aut parum acuto. - Abdomen fominæ oviducto ensiformi, et maris, appendicibus duabus filiformibus longis instructum.

Genus 5. DAEMANWA EREV.

Spec: Typ: Myopa Punctata Fabr.

STraps XI. SIPHONINA Rndn.

racainariat Macq: Westw. Zell. Mgn. - henatomyzides Fall. Zelt. - емтомовіљ R.Desv.

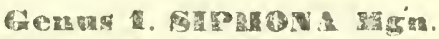

Spec: Tip: Musca Gencerita De-geet.

Stigis XII. TACHIVINA Rodn.

tacuinarie Macq. Zelt. Meig. Westw. - zoobre entozobia Desv. - ocypteratz Macq. - tacuisaRIDE Bigot. - Tacunides Walk.

A. Feno longitudinales quarta el quinta sejunctim costalem 
attingenles, rel raro in costali ipsa conjuncta sed non ante (

7. Vena secunda Iransversaria magis distans a cubito quintix: longitudiualis quau a prima transversa; si raro æquidistans, lunc aris'oe articulus secnndus magis vel minus set distincte elongatus, el cubitus vene quinto longitutualis appendicula nulla neque spuria proditus.

C. Oculi onnino nudi. Articnli duo primi arista breves, vel penuhimus tantum paulo elongatus.

D. Yeno longiudinales quarta et quinta in costali non conjunclat; pirma el secunda in costali parum distantes W. Vena quinta longitudinalis integra, seu ad cubitum non obsliterata.

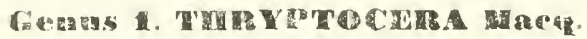

\section{Species Typ: Tagusa Pllipenais Fall.}

LE Tera quina longitulinalis al cubitam obliterata.

I. Vena secimdit transversaria selt exterior non obliterata. G. Ariste anticulus secundus brevis. - Abdomen setis validitisculis erectis non istructum.

\section{Genus a gandA Dew.}

Spec: Typ: l'acuina Isucoptera Meig.

rif. Ariste apliculus securdus magis vel minus elongatus. - Abromm setis valiliusculis erectis preditum.

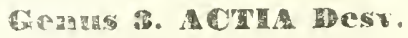

Spee: Typ: (Nova) Vithenenrs Milli. 
FF. Vena secunda transuersaria obliterata.

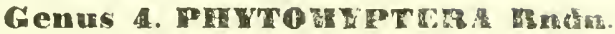

Spec: Typ: (Nova) Privt: Nitidiventris Rnản.

DD. Vence longindinales quarta el quinta in costali conjumclæ (In individua aliquo abnormi, anie costalem). - Vena longitudinalis secunda salis longe ab apice primæ costalem allingens.

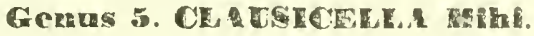

Spec. Typ: (nova) Ceacs: Sutrata Mirit.

CC. Oculi pilosi vel distincte tomentosi, Aristo articuli primi vel ambo breves rel ambo elongali.

H. Aristo articuli dno primi breves. Fono longitudinales quarta et quinta in costali conjunsto.

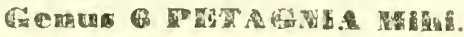

Spec: Typ: (nova) Petagn. Ocglesa Mihi.

int. Aristoe articuli doo primi satis elongati. - Vence longitudinales quarta et quinta sejunctim costalem atirgentes.

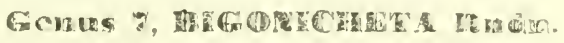

Spec: Typ: Tachira Setipennis Fall.

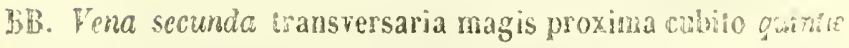
longitudinalis, fuam primce transtersariat, si rato circiter nequidistans, tunc aristce artict32us sectutitus brevissimus, aut cubitus vence quinte longitudinalis appendice saltem spuria zoreditos. 
6?

I. Ariste arliculus secunu'us valde elongatus, longitudine saltem dimidia tertii, et si paulo brevior, unc facies inflata et setis pluribus in genis prædita.

K. Arista articulus secundus longissimus. - Fracies inclinata, non manifeste inflata et setis pimibus oralibus ultra apicem antennarum in facie ascendentibus.

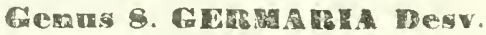

Spec: Typ: Tacuna Rufigeps Fall.

KK. Facies non inclinata et distincte inflata. Setio orales ultra apicem antennarum non ant vix ascendentes.

I. Antennarum articulus tertius saltem trilongior secundo in maribus, et bilongior in foeminis. - Agriste articulus secundus saltem longitudine dimidia tertii aut parum minor. - Pulvilli tarsorum sæpius brevissimi in .utroque sexu, vel vix in mare longiores.

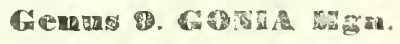

Spec: Typ: Goria Capitata Mgn.

LL. Antcrnarum articulus tertius ad summum bilongior sccundo in maribus, et fere sub-nequalis in foeminis, et si paulo longior, tunc. Arisice articulus sccunters brevior medietate tertii. - Pulvilli tarsorum in mare distincte longiores semper.

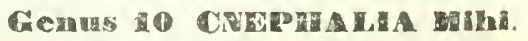

Spec: Typ. Gona Hebes Mgn.

II. Ariste articulus secundus value brevior tertio etiamsi 
paulo elnngatus, et quando talis, tunc facies non inflata et setis plurimis in genis non prædita, raro duabus vel tribus tantum.

A. Antennarum arliculus secundus longior ertio, si raro sub-œqualis saltem in uno sexu, tunc aristce articulus secundus manifeste elongatus et etiam primus paulo longus.

N, Palpi manifesti, et longitudine ordinaria.

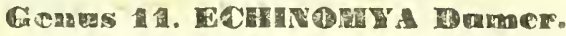

Spec: Typ: Tacenna Fera Lin.

RN. Palpi brevissimi, et extrinsecus non manifesti.

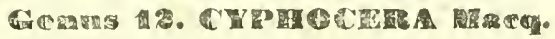

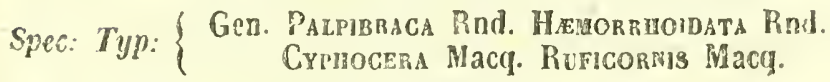

hil. Antennarum articulus secundus magis vel minus brevior tertio, si raro subæequalis, tunc ariste articultis secundus brevis vel brevissimus; primis non aut parnm distinctus.

0. Oculi hrti aut saltem distincte tomentosi.

P. Palpi brevissimi, extrinsecus non manifesti.

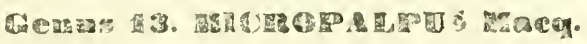

Spec: Typ: Tachina Vulpina Falt.

pr. Palpa iongi vel longissimi, plus minusve extrinsects manifesti.

Q. Antennce contra medium, et non manifeste supra medium oculorum inserta. 
R. Ablominis segmenla iniermedia macrochctis in dorso narginalibus tantum prodita.

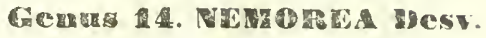

\section{Spec: Typ: Tach. Pellucida Mgn.}

RR. Audominis segmenta iniermedia macrochetis in dorso marginalibus et discoidalibus.

S. Spinula costaiis brevissima sub-indistincta vel parum manifesta. - Tarsi antici in fæmina distincte dilatali.

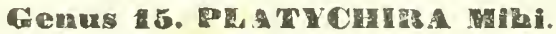

Spec: Typ: Musca Puparum Fabr.

SS. Spimula costalis plus minusve distincia et elongata Tarsi nulli in fæmina manifeste dilatati.

T. Cubitus vena quinto longitudinalis, appendice venosa (non apparenter appendiculatus) longa ef rocta prozditus.

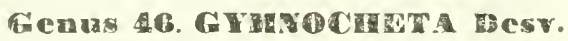

Spec: Typ: Tachixa viradis Mgn.

T'T. Cubitus venæ quintæe longitudinalis non appendiculatus, vel raro appendicula brevi et retro inclinata proditus. 1. Reriscomium relro setas orales * subnudum. Spinula costalis valida - Maris oculi in fronte non contigui. Comas

Spec: Typ: Tachima Treuca Mgn.

Setie orales, qqu aperturarn oralem cingunt. 
UU. Peristomium retro macrochetas orales setigerun vel dislincte piligerum - Spinula costalis medioeris Oculi maris in fronte contighti.

\section{Geras 18 macedatia besw.}

Spec: Typ: Tachina Chalcuonata Mgn.

QQ. Antenna supra medium oculorum manifeste insertie.

V. Cubitus vence quinte longitudinalis non appendiculatus distincte, neque appendice spuria (nisi sat raro in individuo aliquo abnorme).

X. Tarsi antici fæminæ satis dilatati - Maris pulvilli pt unci tarsorum breres it in fæmina.

\section{Genus 10. Wherista}

Spec: Typ: Tachima Simpleitaras Zetl.

XX. Tarsi antici in fxmina non dilatati - Maris pulvilli et unci tarsorum sat Iongiores.

\& Scrics orales setarum ulira medium faciai non ascentdentes in mare et fomina.

Z. Aristce articulus peunltimus manifeste longiusculus et cum tertio sape cubitatus; antennisque simnt articulo secundo lingiore medietate tertii.

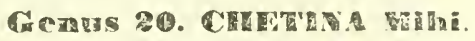

Spec: Typ: (nova) C. Setigena Mihi.

Z\%. Aristce articulus penultimus brevis, et si aliquando lon-

(") A quo forsitan sejungenda spec: nova, Brevifrons Mihi, quæ generice ab aliis distincta erit - Fronte nihil omnino proeminente Peristomio setoso, prieter macruchetas orales ect. - Geuus apellandum TuRrchogras Mibi. 
giusculus, innc antennarum articulus secundus satis brevior mediclate tertii.

7. Antennarum articulus secundus longior medietate tertii, et simal abdomen macrochelis discoidalibus in segmentis intermediis preditum.

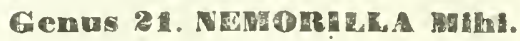

\section{Spec: Typ: Tace, Maculosa Mgn.}

17. Antennarum articulus secundus brevior medietate tertii, et si paulo longior, lunc abdomen macrochetis discoidalibus in segmenlis secundo et tertio non præditum.

W. Abdomen macrochetis discoidalibus in segmentis intermediis destitutum.

\section{Genus 22, CEETDEAG A whi.}

Spec: Typ. Tach: Gava Mggn.

WW. Abdominis segmenta secindum et tertium, aut saltem unum macrochetis discoidalibus aliquibus distinctis in dorso predita, nisi plurimis.

a. Vibrissce * longe a margine supero oris in facie insertæ - Peristomium sub oculos satis elongatum, longitadine circiter dimidia oculorum.

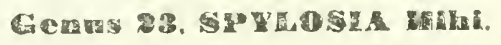

Spec: Typ: Tacinka Bigigmata Mgn.

(") Vibrissa a me apellantar sxla orales aliis mojores quze acpe daz sans el extrinsecns superposita. 
a Vabrissa contra marginem superum oris inserta - Peristomium vix ant parum sub oculos alongatum.

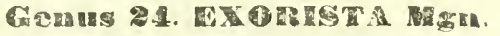

Spec: Typ: Tachina Obscura Fall.

Lf Series oralcs sctarum manifeste supra medium faciei ascendentes saltem in mare, si in fomina breves, tunc adominis segmentum secundum macroc'selis cincturn.

b. Abdominis segmenta omnia in mare macrozhe'is cincta, tria ultima in fœmina. Pulvilli et unci tarsorum in mare parvi un in fæmina. In utroque sexu setæ frontales similes, serics orales setarum in fænina brevissimze.

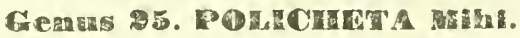

Spec: Typ: $\left\{\begin{array}{l}\text { Unicolor Fall. (Mas.) } \\ \text { Tachina Inequalis Milii. }\end{array}\right.$

b. Abdominis segmenta duo basalia macrochetis non cincta - Maris pulvilli tarsorum el unci satis longiores - Frontis selce in famina plures, series orales ut in masculis circiter in facie ascendentes.

- Facies modice aut parum in medio excavata. Series orales in genis ascendentes simplices.

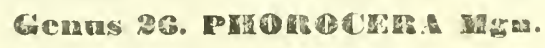

Spec: Typ: Tacir. Punicata. Mgn.

" Sic subdivideadum si placet

a Series orales setarum vis ultra apicem antennar: ascendentes.

G. Exorista Mgn.

wa Scrios orales ad medium circiter articuli 3 antenvar: ascendentes.

G. Blephanidea Mihi.

Spec: Typ: Tachina Vurgaris Fall. 
68

cc. Facies in medio maxime excavata. - Series orales setarum in facie ascendentes dupplicaice.

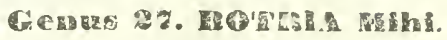

Spec: Typ: nova B. Fsscuoruß Mihi.

YH, Cubitus venœ quinlo appendice spuria sed distincta præditus.

4. Series orales setarum manifeste supra medium faciei ascendentes.

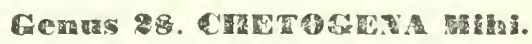

Spec: Typ: Tach, Gramua Mgn.

da. Series orales setarum in genis parum ascendentes, aut saltem ad medium faciei non productæ.

e. Series frontales setarum salis longe a peristomio sistentes.

- Vena transversa exterior modice aut parum obliqua

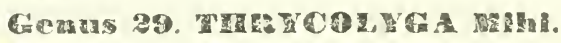

Spec: Typ: T. Nova Mihi.

ce. Series frontales setarum fere usque ad peristomiun descendentes. - Venatranstersa exterior valde obliqua.

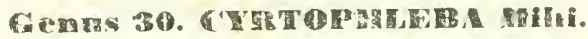

Spec: Typ: TAciz. Ruricola Mgn.

00. Ockli non manifeste pilosi.

z. Cubilus venæe quintæe longiludinalis appendice longa saltem spuria præditus.

P. Series frontales setarum ultra apicem articuli secundi antennarum in facie descendentes. 
4. Series frontales fere usque ad epistomim modnctax.

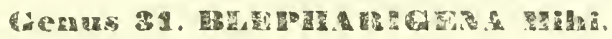

Spec: Typ: Tachivd Trepida Mgn.

6. Series fromales ab epistomio satis distantes.

๘. Fena transversa exterior satis obliqua. - Antennarum articulus pentlimus partum brevior ialinino.

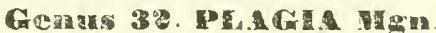

Spec: Typ: Tachina Verticalis Mgn.

ieka. Vena transtersa exterior parum ant vix obliqua. - Intennarum arlicnlus penullimus salis brevior ultimo.

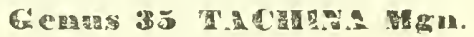

Spec: Typ: Musca lanvarun Lin.

r. Series fromtaies setarnm vel ad radicem antemnarum si, stentes, vel ad summum usque ad apicen articuli sectiadi antennarum et non ultra productæ.

i. Series fronlales setarum usque ad apicem articuli secundi antennarum circiler produclat.

k. Abdominis segmentmu tertium macrochetis postice cincturi.

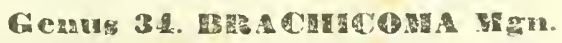

\section{Spec: Typ: 'T'achisa Nitidula MIgn.}

5k. Abdominis segmentun tertium uacrochetis dorsnalibuduabus el aliquibus lalera!ibus tantum.

1. Arista nuda.

m. Vena transversa exlerior parum oblinga el postice lonse" ab incrioni orjens. - Cubitus vener Inngitulimalis 
quintæ appendice præultus satis breviore parte ultra cubitum venæ ipsius.

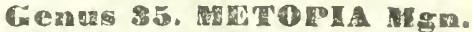

Spec: Typ: Musca Leucocephala Rossi.

wn. Vena transversa exteriol salis obliqua et postice oriens contra interiorem. - Cubitus longitudinalis quintæ appendice longissina præditus, longa circiter ut pars ultra cubilum venæe ipsius.

\section{Cenus 36. How}

Spec: Typ: Miltogramia Heteroneura Mign.

11. Arista in medio paulo pilosula.

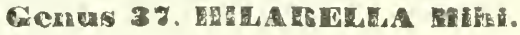

$$
\text { Spec: Typ: }\left\{\begin{array}{l}
\text { Hulabelia Zetterstedtu Mihi. } \\
\text { Miltograma Hilarela Zell. }
\end{array}\right.
$$

11. Series fromales setarum usque ad radicem tantumantennarum productæ.

4. Appendix cubitalis venæ quintæ spuria. - Abdomen macrochetis marginalibus tanlum in dorso sugmentorum intermediorum.

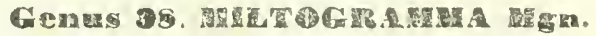

Spec: Typ: M. Conica Mgn.

na. Appendix cubitalis veræ quintæ longituuinatis non spuria rel nulla. - Abdomen macrochetis margiaslibus el discoidalibus instructum.

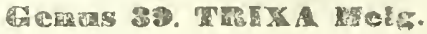

Spec: Typ: Trixa Carulescess. Mgr. 
$\therefore$. Cubitus vena quala longitadinus non distincte appen-diculatus. (appendicula vix perspicienda in individuo aliquo abnorme).

*. Series frontales setarun in genis, magis vel minus descendentes, sallem ullra apicem articuli primi antennarum distincte elongata.

- Vena quinta longitudinalis angulatim sæpius et non arcuatim flexa, et si cubito paulo rotundato tunc serics frontales setarum dislincte magis remolæ ad apicen, a carinis facialibus quam ab oculis.

. Tibie postice cxlrinsecus setulis brevibus aqualibus el crebris cilia!æ.

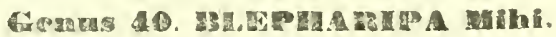

Spec: Typ: Masicema Ciliata Macq.

pp. Tibra postica selis crassitice el longitudine variis extrinscens præditæ.

q. Series faciales selarum parum in genis, vel ad summum prope inedium facies ascendentes, et ideo a fromalibus satis distanles.

. Yena quinta longitudinalis supra cubitum nec exilissima et semi-spuria, nec interrupta.

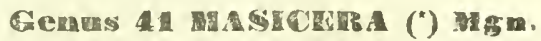

\section{Spec: Typ: Masigera Seniuls Meig.}

(1) Genus boe sic dividendum si placel:

a Abdomen macrochetis tantum marginalibus in dorso seguentusum intermediorum. Masicera Mga.

as Abdomen mecrochetis discoidalisus et marginalibus. 
rr. Vena quinta longitudinalis supra cubitum vel interrupta vel exilissima el sentispuria.

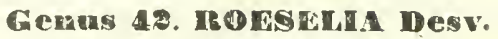

Spec: Typ: Tachina Antiqua Fall.

114. Series faciales setarum in genis valde ascendentes et usque ad frontales aut parum ante productæ.

Gemas 43. Froving Meig.

Spec: Typ: Frontina Demissa Mgn.

o. Vena quinta longitudinalis arcuatim et non angulatin sæpius flexa, et si cubitus paulo angulosus, tunc seriesfrontales setarum, ad apicem magis proximæ carinis facialibus quam oculis, aut ad summum æequidistantes.

s. Series faciales setarum satis ultra medium genarum ascendentes.

Geras 4. DEG

Spec: Typ: Tachina Coiraris. Fall.

ss. Scries faciales setarum ad medium genarum non ascendentes.

๔. Macrochetce aliqux in dorso segmenti secundi validxe et erectæ adsunt saltem marginales.

a. Proboscis non aut vix producta ultra epistomium. Antennarum articulus tertius bilongior secundo.

\section{Gemus 45. MYOBIA (") Desv.}

Spec: Typ: Tachiva Pacifica Mgin.

(i) In Juo subdivideudum Genus hoc si piacet, sic:

a Abdomea dorso macrochetis discoidalibus et marginalibus. Mrobia Desv. wa Ibdomen macrochetis marginalibus tantum. Bromya arihi. 
ag. Proboscis satis monbela ultra epistominm. - Antennarum articuli secundus et rertius snbarque longi.

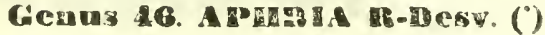

Spec: Typ: Tacuina Longrostr:s Meig.

38. Macrochetce abdominis in segmemo secundo sub-indistinclx et non erectæ.

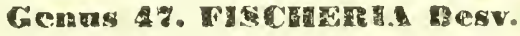

Spec: Typ: Fiscireria Bicolor Desv.

Xath. Series frontales setarmm non ant vix ultra raticem aniennarum productx, al stummum usque ad anicem articuli primi antenuarmu, sed non ultra.

- Abdomen foeminx apice non forficularo.

*y. Vena secunda longitudinalis costalem allingens satis ultra transversariau anteriorem. - Abiomen man is genitalibus non elongatis et subtus nou incurvatum.

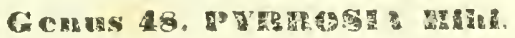

Spec: Typ: Tacina Aured Fall.

wena secunda longiludinalis costatem allingens contra circiter transversan anteriorem; et si aliquando paulo ultra, tunc abdomen maris sublus incurvatun, genitalibus plus minusve elongatis.

$\mathrm{x}$. Abdomen macrochetis dorsualibus erectis nudlis, sed pilo-

(7) Ragaphisa Macq. Rudn. 18 is Anis. Bulugna.

Raschosia Matq. 1898 Ao: Soc: Eutoca. 
sum tantum. - Vibrissa in genis insertx satis longe a margine supern oris.

Genus 40. MHyccomy Dest.

\section{Spec: Typ: Tacuna Columbias Mgn.}

xx. Abdomen macrochetis saltem aliquibus validis ef erectis præditura. - Vibrissa inserlæ contra marginem superum oris.

- Abdomen subius distincle incurvatun, aut saltem genilalia calis longa el subtus flexa.

J. Vena transurrsaria secunda magis distans a prima, quam

a cubito quinta longitudinalis.

2. Vena longitudinalis secunda uitra transversam anteriorem satis producta. - Arista articulus secundus dislinte longiusculus.

\section{cenus 50. Pusvosona wh.}

Spec: Typ. (Nova) Pranuosoma Apennina Mihi.

22. Fena longiludinalis secunda contra primam transversa- riam costali conjuncta. - Aristce articulus secundus brevissimus.

1. Ani crnarum articulus tertins bilongior aut ultra secundo. Genus 51. Dha Nia Meig.

Spec: Typ: Puania Obscuripennas Mign.

AA Antennarum articulus secundus parum brevior tertio. Genus 52. MEDORERA wihi.

Spec: Typ: (Nova) Medorula Subfasciata Mihi. 
5y. Pena transversaria seeunda xequidistas circiter a prima et a cubito quinte longilmdinalis.

\section{Genua 63. UROuYA helg.}

\section{Spec: Typ: Tachina Cunvicauda Fall.}

*w. Aldomen haud distincte incurvalum: - Genitalio non distincte elongata et snblus flexa.

- Antennce supra medium oculorum distincte insertæ.

c. Antenna ad apicem extrinsecus mucronalse.

\section{Gentas 54. AChuY Dew}

Spec: Typ: Acerry Subroturda Desv.

cC. Antenna ad apicem non mucronatx.

- Ancennce articulo tertio latissimo subirigono

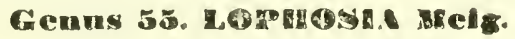

Spec: Tyz: Lophosia Fasciata Meig.

oj. Antenna latiludine et forma ordinariis.

- Oculi maris in fronte distantes circiter ut in fomina, seu paulo minus - Fomina amus non forficulatus.

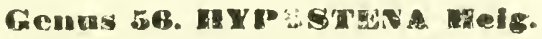

Spec: Typ: Ilyfostena P'rocera Mgn.

*2. Oculi maris in fronie subcontigni. - Anus fomina forfic ulatus.

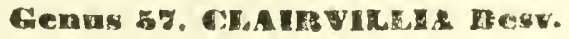

Spec: Typ: Crankvilula $\left\{\begin{array}{l}\text { Dispar Rodin. } \\ \text { Pusilsa Desv }\end{array}\right.$ 
E. Anserna conita, nisi sub medirm nculorun inserla.

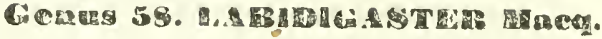

\section{Spec: Typ: Carma Minor Desp.}

AA. Vence longitulinales giraria et quinta magis vel minurs longe a covld'i conjanc! 2 . In individuis aliquibus abnor-. mibus, raro in costali conjuncla ei rarissimo sejuncto).

F. Vena transersaria secunda, magis distans a prina quam a cubifo raina longinulinalis; si raro fere aquidistans, tume scries fronules setarum in genis descendentes saltem nsque al apricem arliculi secundi amlennarram.

Go Abdomen fenine forfictild frecta apicali præedituro. - Genitalia maris perrecta et sublus flexa.

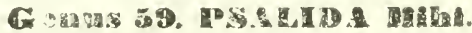

\section{Spec: Typ: Taguna Leucostoma Mgn.}

ซg. Abdomen fenine furfeuls destitutrm. - Genilalia maris non elongata nec subens flexa.

x. Series frontales setarum in genis descendentes usque ad peristomium.

7. Antennce brevissimæ, articulis secundo et terlio fere æque longis.

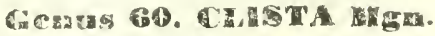

Spec: Typ: Cuspa Moraens Meig.

13. Antchno magis rể mints clongalæ; articulus tertins sal tem bilongine ce ultra secindo.

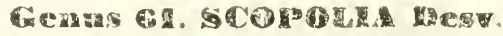

Sp?c: Typ: Thama luans Meig. 
ux. Series frontales setarum ad summum in meßio tanturt fuciei descen!entes.

x. Vena quinta longitudinalis arcuatim non angulatim fex.t

- Proboscis disticicte pruducta nJira episiomium, stú nec fliformis, nec longissima.

\section{Genus 63. Hatchessa min.}

Spec: Typ: (nova) Istogrossi Puelza Mihi.

4x. Vena quinta longitudinalis angulation non arcuatian flexa.

- Proboscis non dislincte ultra ppistomium productá, vel in unico genere filiformis el longissima.

2. Series faciales seta!um ad medium circiter genarum ascendentes.

a. Gence proler selas ordinarias nec piligere nec seligera,

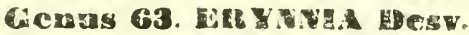

Spec: Typ: Erymina Petiolata Desv.

w. Geno praier sclas ordinarias distincle setigerso

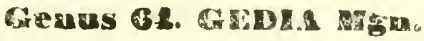

Spec: Typ: Tacaina Conkexa Meig.

2u. Series faciales selanum brevissims, seu a medio genarum distantes.

x. Proboscis nififormis et Inngissima.

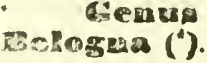

Spec: Typ: Stomozis Pedudcitana Meig.

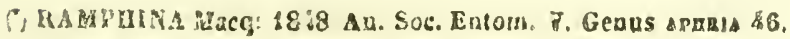


w8. Proboscis nec exilis nec longissima.

๑. Oculi manifeste pilosi.

\section{Genus 66. OLIVIERIA Dewe.}

\section{Spee: Typ: Tachina Lateralis Mgo.}

oo. Oculi nudi.

P. Palpi brevissimi exlrinsecus non manifesti.

p. Vena transversaria secunda recta, et subperpendicularis pracedenti. - Arista maris apice incrassata. - Pulvilli et unci tarsorum in utroque sexu parvi.

Genus Gg OCXPTERULA whi.

Spec: Typ: Ocrptera Pusilca. Meig.

QQ. Vena transversuria secunda obliqua et sinuosa. Arista in utroque sexu ad apicem non incrassala Pulvilli et unci larsortun in mare satis majores.

R. Abdomen subeylindricam, inferne non dilatalo-cariadtum - Oris margines setis ciliali sub vibrissis duabus ordinariis decussatis.

Genus 63. oCYPTEIA Latr.

Spec: Typ: Ocyptera Brassicaria Fabr.

яв. Abdomen inferne dilatat(1-carinatum - Os setis dnabus tantum vibrissinis instructum.

\section{Cenus 69. WIOGASTER Mih.}

Spec: Typ: (nova) Exog aster Carinatus Mihi.

FP. Palpi modo orlinario elongati, et extriusecus magis vel minus manifesti.

s. Cubillos yen quiate longitudialis appendice longa pra- 
ditus - Tarsi antici remiformes, sea dilatalo-compressi.

\section{Geung \%o. Hisนก Dew.}

Spec: Typ: Dexia Coupressa Meig.

84. Cubitus, venæ quintæ Iongiludinalis appendicula nulla vel brevissima el subnulla - Tarsi non remiformes.

3. Oculi maris in fronte sub-conligui - Pulvilti et unci tarsorum anticorum in forminis nulli, seu indistincti. Gence utrinsque sexus præter setas frontales et faciales non setigeræ.

\section{Genus 7. Cyundorosona hith.}

\section{Spec: Typ: Tacuira Sanguivea Meig.}

צт. Oculi maris in fronte distantes $-p_{\text {ulvilli et unci }}$ tarsorum anticorum in fæmina distincti - Gence in ntroque sexu proter setas ordinarias piligere vel setiger:x.

\section{Genu. כ2. מบIYTO Dew.}

Spec: Typ: Tacina Paivicornis Meig.

wr. Vena transversaria sccunda magis proxima primx, quam cubito quinta longitudinatis - Series frontales setarum non, aut vix ultra radicen antennarum productæ.

i. Yera quinta longiludinalis angulatin el non arcuatian nexa - Abdomen sublus non incurvatum.

r. Gence proter setas froniales et faciales piligera - Ab 
domen selis marginalibus el discoidalibus instructuas

- Oculi maris in fronte subcontigni.

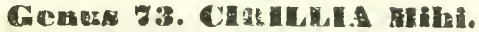

Spec: Typ: (nova) Cirlulia Ancustifnons Mihi.

vv. Gence nudæ - Abromen setis marginalibus tantum instructum - Oculi maris in fronte distantes.

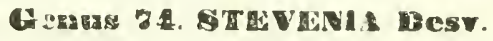

Spcc: Typ: Ocyptera Miaculata Fall.

uv. Iena quina longiludinalis arcuatim flexa - Abdomen subus incu: vatuan.

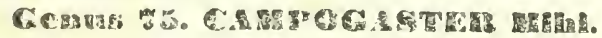

Spec: Typ: (nova?) Casp. Parvelus Mihi.

Strops XIII PIIASIINA Rndn.

phasianeg. Desv. Macq. Westw. Mrig. - nersomyzes Fall. - priastarite. Zell. - inaside Rigot. - Gimosoméc Macq. Wesiw. - Gastrode Desy.

A. Vence longiludinales quarta ef quinta ante costalem vel in costali conjunctæ.

R. Abdomen dorso quamvis breviter, tamen distincte piligero et seligero. - Carina faciales selis exilibus sed pluribus cilialæ. 
C. Esta quinta longitudinalis quartx conjuncia anic costalem, in summo apicr intus non rélixam.

\section{Cen: 1. ALOPHOR. Desv.}

Spec: Typ: Tuereva Cinerea Fabr.

CC. Pena quinta longitudinalis in costali conjuncta; cos'alis summo ajice iulus reflexa

\section{Gerus 2. HLOny Desv.}

Spec: Typ: Pasia Lateraus Meig.

BB. Abdomen dorso omniun nudo. - Carine faciales pilis el setulis oumino vel fere omnino destitutæ.

D. Antennce elongald uip!te ad orem, articulo tertio non rolumdato sed elongato.

\section{Geans 3. Gravosoni Melg.}

Spec: Typ: Musca Poturdata Lin.

aD. Antennce brevissian et ab ore satis distantes, articulo ter tio subrotundalto.

\section{(deaus 4. CISTOGASTEZ Latr.}

Spec: Typ: Gynnosoma Dispar Fall.

AA. Vence longludinales gutarta el quinta sejunctim costalem allingentes.

色. Abdomen dorso modo vel subnulo.

F. Carince faciales nec setis nec pils distinctis ciliatx

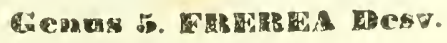

Spec: Typ: Frerea Gagrtea Desy. 


\section{2}

5F. Carince faciales setis ralidis instructæ.

\section{Genus B. Xist. Hetg.}

Spec: Typ: Xista Cilipes Meig.

EE. Abdomen dor'so saltem breviter sed distincte piligero vel setig, ro.

G. Series fuciules setarum in genis satis ascendentes -

Peristumium non setosum - Abdomen setis margiDalibus sesmentorum exilibus et flexis, seu non erectis.

denus \%. PHABIA Mels.

Spec: Typ: Puasia Teniata Pauz:

GG. Series faciales setarum brevissimæ - Peristomium st tosum - Abdomen setis marginalibus validiusculis el erectis.

H. Antenna brevissima, articulo tertio subrotundato et parum diverso longitudine a præcedente.

Gemus 8. ChNTha Dew.

Spec: Typ: Tacuina Helvola Mgn.

HR. Antenna fera usque ad orem elongate; articulas earace ultimus duplo ef ultra longior pracedente.

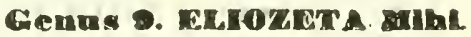

Spec: Typ: Tacuin-Pellucens Mgn. 
StikPs XIV. DEXINA Rndin.

Dexiakie Dest. Macq. Zetl. Meig. - sacophagn. Desv. Macq. - theramida Desv. - sarcophagiobs Walk. - Sarcoptugarte Zell. - dexides Walk. sarcuphage. Westiw. - iextde Bigol.

A. Gena in parte supera, ut fions tota pilis crebris tecta ommino.

\section{Genus 1. MOnphosy Hitus:}

Spec: Typ: Musca Tacunoides Fall.

A A. Gence in parte supera, ut magis vel mimus late etiam frons, nudx vel subnudx.

B. Tibio posticœ, xștrinsecus setulis crebris et aqualibus ciliata.

c. Facies inter antennas carina distincte elevata usque ad earnm apicem - Antennarum articulus tertius bilongior circiter secundo - Abdomen segmento secundo macrochetis destituto. .

\section{Gemus 2. MYOSTOMA Desy.}

Spec: Typ: Myostoha Mrgrocera Desv.

cC. Facies in medio antennarum non manifeste carinata rel carina brevi et parum distincta prope earum basim - Antennarum articulus tertius circiler trilongior secundo - Abdomen segmento secuado sacrochntis aliqnibus predito.

Cenus 3. Phrokostroma Desv. 
Spec: Typ: Puorostora Subotumdata Desv.

BB. Tibia postica selis varie elogantis ef now crebris in parte extrriniti prerlitio.

D. Carina distincte elevata inter antennas panto remotas.

E. Vence longitudinales quarta et quiuta in costali vel ante costalem conjunclæ.

F. Spinula costalis valisla - Vence longitudinales quarta ef quinta in costali conjuncta.

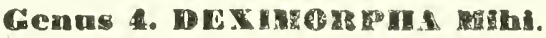

Spec: Typ: Dexia Mariturua Macq:

FF. Spinula costalis vix dislinguenda - Vence longitudinales quarta et quinta paulo aute costalem conjunctx.

\section{Genus 5. Diveren Desv.}

Spec: Typ: Musca Grisescens Fall.

EE. Wence longiludinales quarta et quinta sejunctiro costatem altingentes.

G. Proboscis exilis et longissima.

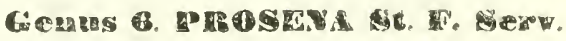

Spec: Typ: Stomoxis Cinebea liabr.

GG. Proboscis pasum aut non producta ultra epistomiura. Gerass.

Spec: Typ: Dexia Carivifrons Fall.

aD. Facies inter antennas subcontiguas non manifeste carinala.

II. Vence longitudinalis quintæ cubitus appendice præditus aon spuria et longa. 
1. Vence longitudinales quarta el quinta anie costalem coujunctæ.

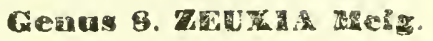

Spec. Typ: Zeuxia Cinerea Meig.

II. Vena longiiudinales quarta et quinta sejunctim costali produciæ.

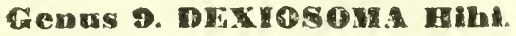

Tpec: Tyn: Dexia Canina Fabr.

71H. Cubitus venæ quintæ longitudinalis appendice vel mulla vel spuria.

K. Cubitus renæ quintæ longitudinalis appendice spurias prædilus.

- Vena transuersaria sechnda magis distans a prima, quama a cubito longitudinalis quintæ - Antennarum ariculus secundus duplo saltem breviug tertio.

1. Tibia antica macrochetis validis extrinsecus totre ciliatx - Sculcllum pre!er macrochetas mar.ginales selis validis pluribus in disco preditum.

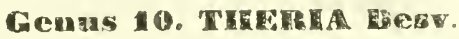

Spec: Typ: Sarcopaga Muscaria Mign.

111. Tibice antica selis pancis et sparsis sed macrochets validis non lotæ ciliałæ. - Scutellum præeter macrochetas ordinarias marginales, selis validiusculis duabus vel nullis in lisco predilum.

N. Unci ef pulvilli tarsorum in maribus manifeste majores. 
- Frons maris angusla el seriebus duabus taniua setaram instrucla, focmina seriphus quatuor.

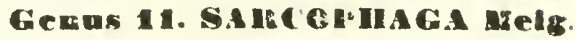

Spec: Typ: Sarcophaga Carnaria Fabr.

NiN. Unci et pulvilli tarsorum in mare et fœmina parvi. Frons in utroque sexu lata el seriebus quatuor selarum instructa.

\section{Genus 12. S IHCOPIILA Mihe.}

Spec: Typ: Musca Latifaons Fall.

U. Tena securda transversaria circiter rquidistans a prims et a cubito quinta longitudinalis. - Antennarum articulus tertius parum longior secundo.

0. Tance longitudinales quarta e quinta sejunctim costali producle.

\section{Cenus 13. NyCtAa Desw.}

Spec: Typ: Tachina Maura. Meig.

0. Vence longitudinales quarta el quinta conjuncta ante costalem.

\section{Gents 14. MEGRERE Desy}

Spec: Typ: Tacuina Caminaria Meig.

KK. Cubitus vena quintæ Jongitudinaiis neque apparenter appendiculatus.

P. Vence longitudinales quarta el quinta conjunclat ante costalem.

Q. Amennce supra medium oculorum manifeste inserta. - 
Gence inter oculos et antennas pilis vel setulis validinsculis prædiize.

\section{Genus 15. Pracocuis Desw.}

Spec: Typ: 'Tacuina Atramentabia Mgn.

QQ. Antennce in linea fere suh-oculari insertæ. - Gence to ter oculos et antennas non setigeræ.

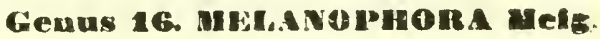

Spec: Typ: Musga Roralus Lin.

P1. Vence longitudinales quarta el quinta sejunctim costalem altingentes, vel raro in costali et non ante costalese conjunclæ.

A. Antenna in linea fere sub-oculari insertæ; articulis cundo et terlio longitudine sub-aqualibus.

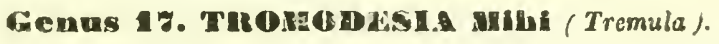

Spec: Typ: (nova) Tromodesa Vibripenwis Mihi.

RR. Antenno contra vel supra mediun oculorum inserta, articulus ultimus carum saltem bilongior præcedente. S. Abdomen fœminæ durso segmenlis iribus basalibus non seligeris. - Capul maris vertice macrochetis longis destituto.

\section{Genus 18. ANTHRACOMY Mih.}

Spec: Typ: (nova) Anturaconya GenèJ! Mim.

5S. Abdomen fominæ sallem in dorso segmenti tertii selis distinctioribus instructun. - Caput maris rertice rus srochetis quatuor præditum. 
88

T. Yere longitudinales omnes all basim haut setuloso-eiliatæa, aliquando tantum vena quarta ad busim setulas e?!quess plwhe?.

J. Vena eransversaria secunila maris proxima prima quary cubito rel curvalura quinta longilu linalis.

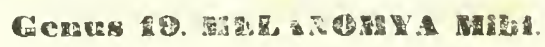

Spec: Typ: Dexa Naxa Meig.

UU. Vena transuersaria secunila rel magis distans a prima gram a cubio quinix longitudiualis, vel ad summura æquidilistans.

V. Ala longiores abdomine, cosia antica non sprantosow serrata.

X. Gence prater setas faciales inter antennas et octios seligera, seu macrochelis instiucla.

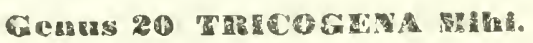

Spec: Typ: Tricogera Trequi Mihi (nova).

XX. Gence preter setas faciales macrochetis non instructi. 7. Oculi nudi. - Proboscis non distincte producta ulum epistomium.

Genus 21. Merager.

Spec: Typ: Dexia Anturacina Mgn.

2. Ocali distincte tomentosi. - Proboscis distincte produtive ulira epistonitum.

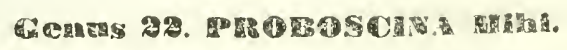

Spec: Typ: (nora) Proboscina Longires Tilbir 
ry. Ale bicriores abdomine, et costa selubso-serrata.

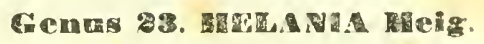

Spec: Typ: Tachina Volvulus Fabr.

TT. Vens longitudinales secunda et quarta ad basin late sctuloso-ciliatæ.

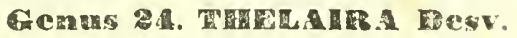

Sire: Typ: Dexia Nigripes Fabr.

Stirips XV. MUSCINA Rndn.

Muscie Macq. Westw. - ruscide Desw. Bigot. uuscaria Zell. - Muscine Meig. Rndu. - musciden Walk. - Muscee Westw.

A. Tibice intermedia intus seta aliqua valida instmetie.

B. Ariste pars nuda apicalis satis longior uhtimis pilis quibus prædita est.

C. Abtomen segmento secundo pilis distinctis et setis in dorso destituto. - Oculi maris in fronie distantes.

cenus 1. CYworar Bes:

Spec: Typ: Hesca Mormorum Fabl. 
CC. Abdomen sfinnento secundo pilit distinctis vel selis dor sualibus instructo. - Oculi maris in fronte sub-conligui.

\section{Genus 2. O.VEST IDEv.}

Spec: Typ: Musca Ves̀pllto Fabr.

BB. Arisice jars nuda apicalis non atit vix longior ullimis pilis quibus prædita est.

D. Vena quina longitudinalis angulatim non arcuatim flexa.

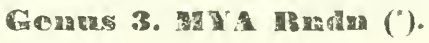

Spec. Typ: Musca Vomtona Lin.

DD. Tena longitudinalis quinta arcuatim non angulatiu flexa.

E. Vena longiludinalis secunda costalem attingens parum ultra transversam primam et satis ante secundum. F. Oculi nudi.

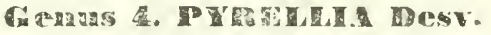

Spec: Typ: Milsca Cadaveruma Lin.

FF. Oculi pilosi seu tomentosi.

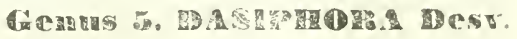

Spec: Typ: Mesca Pratorum Meig.

(1) In gucsto genere ho incluso le specie dei generi di Robineau, Calliphora, Lucilia e Pollenia perchè non mi è stato possibile di riscontrare carattcri importanti e sicuri che valessero a distingnerti. 
EE. Vena longitudinelis secunda vahe producta in costali ullra tiansversam prinam et fere contra secundam.

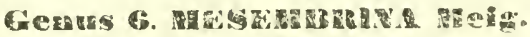

\section{Spec: Typ: Musca Menidiaxa Lin.}

A. Tibice intermedioe inlus setis validis destitultr.

G. Vena longitudinulis quinta a!cuatin et non angulatim tlexa.

II. Oculi nudi.

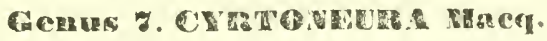

Spec: Typ: Musca Pasceoren Ein.

III. Ocili pilosi.

1. Anternce in facie distantes, carina intermedia distincte elevata.

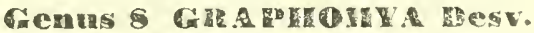

Spec: Typ: Musca Macilata Fabr.

II. Antenno in facie approximatie, carina internedia nou distincta.

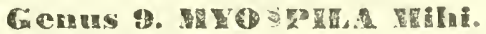

Spec: Typ: Musca Meditabunda Fall.

GG. Vena longitudinalis quninta angulatim non arenatim flexa, angulo suepe paulo rotundato.

K. Oculi nudi.

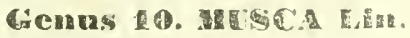

Spec: Typ: Musca Donestica Lin. 
Kh. Oculi pi!osa.

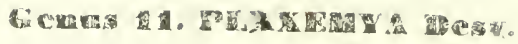

Spec: Typ: Mrsca Viteipennis Meig.

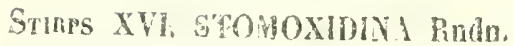

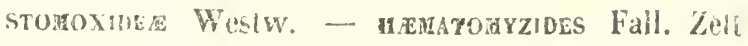

- Muscie Macq: - arescioe Desa. - ruscine Meig.

A. Proboscis inlua epistomiun non producta. - Epistominu distincie porrectun.

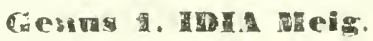

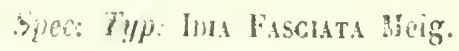

A A. Proboscis distincte prometre ultra epistonium. - Enislomium pärtm ant sic porrectum.

B. Palpi bueres et exiles.

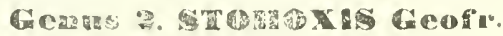

Sipre: 7yp: Becentes Caccirsus Fabr.

BB. Palmi elongali fere ni proboscis et paulo dilatatocompressi.

C. Arista pilis aliquibus interne prodita, superis non undulatis. - Vena longitudinalis secunda costalem attingens satis extra primam transversariam.

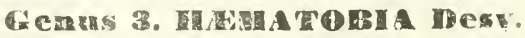

simer: Tym: Srguoxis Stumcans Fall. 
C. Arisca superes tantom pilosa, pilis undulatis. - Yema Tongitudinalis secunda costalem attingens contra prisnam transpersariam.

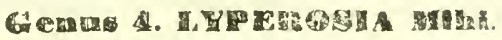

Spec: 7yp: Stomoxis Iraitaks Meig.

\section{STinps XVII. RNTHOMYNA Rndr.}

anthonTyanes Meig. Zett. - hxtdengy zid. Latr. Mact. Bigo: - antuomyde Walk - mesouyne Des.

1. Orbitce (1) oculorum in mare (2) superne contigua vel subcontigure, rel linea tantum exili in fronte segus:ctæ.

S. Squance sub-alares seu Caliptera infera a superis non omnino tecta in ulrogue sexu.

(1) L' orbita oculare è quella linca o fascia che circonda gl' oc.. chi di teli inselti, distiata dalle parti vicine del capo, pel cotore o la lisciatura od altre apparenze.

(2) In questa Stirpe come il alcuse altre seguenti, non si ponno distigguere focilmente le specio ed i generi, se non si conoscunn i tae sessi di ciascuna specie; ma pero interessa piu di conoscere i maschi perchè in geserale si eprogziano ai caratleri di questi le piu sicure e piu Pacili distinzioni speciéche e generiebc. 


\section{1}

C. Femora antica in mare intus dentata vel excavata.

D. Oculi in utroqne sexu nudi.

\section{Genus 1. Hiterothita Besv.}

Spec: Typ: Musca Dextipes Fabr.

DI). Oculi in mare hirti, in fæmina aliqando vix ad lentens tomentosi.

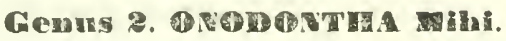

Spec: Typ: Hydrothea Floccosa Maeq.

CC. Femora antica nec dentata nec excavata.

E. Tibia postica in mare intus barbatæ, et in utroque sexul magis vel minus arcuatæ.

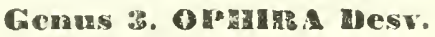

Spec: Typ: Amthonya Leucostonia Meig.

EE. Tibio postice nec distincte barbatæ in mare, nec manifeste arcuatæ in utroque sexu.

1. Proloscis parte apicali fracta et retro flexa.

Genus 4 Hag

Spec: Typ: Musca Hamata Fall.

ij. Proboscis parte apicali nec fracta nee petro fledd.

G. Arista plumata aut distincte pilosa in utroqne sexu. -

Fenoe nulle spinuiusat.

11. Oculi in uroque sexu nudi.

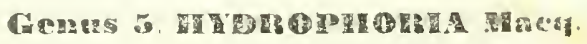

Spec: Typ: Musca Pagana Falre. 
HII. Oculi in utropue sexu pilosi, vel aliquando in femint tomentosi tantum.

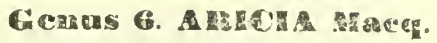

Spec: Typ: Musca Erratica Fall.

GG. Arista suhnula vel pubescens tantum in utroque sexts. et si aliquando in mare paulo pilosula, tune vena secunda longitudinalis prope apicem spiusulis aliquibus validis instructa.

1. Oculi in utroque sexi nudi.

K. Orbila oculorum in mare latiusculæ ideo oculi in frovite paulo distantes - Arista in mare paulo pilosula, in læmina nuda - Vena longitudinalis secunda in utroque sexu prope apicem spinulosa.

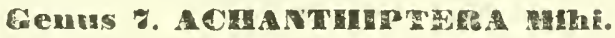

Spec: Typ: Musca Inanis Fall.

KK. Orbitce oculorum in mare exiles, ideo ocnli in fronte subcontigui. - Arista in ufioque sexi nuda vel subnuda. - Vena longitudinalis nulla ad apicem spinulosa.

L. Vena analis etiamsi ad apicem spuria tameu fere rel usque ad marginem posticum alarun elongata.

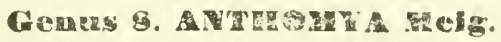

Spec: Typ: Musca Radicum Lin.

LL. I'na analis satis brevis, seu longe a margine postico alarum sistens.

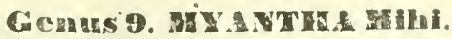

Spec: Typ: Musca Cantculants Lin. 
II. Oculi distincte pilosi saltem in mare.

\section{Genus 10. HAStops Meig.}

Spec: Typ: (nova) Lasiops Antuorynts Mihi.

BB. Squama sub-alares seu caliptera infera a superis omnino tecta, rel vix linea marginali observanda.

M. Arista nuda ve! subnuda in uroque sexu.

$\mathrm{N}$. Oculi pilosi saltem in mare.

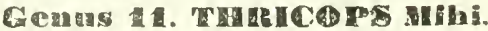

Spec: Typ: Arluia Hirtula Zell.

NN. Oculi nudi in utroque sexul.

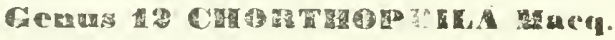

Spec: Typ: Antuonya Szpia Meig.

MM. Aristo plumata ant distincte pilosa.

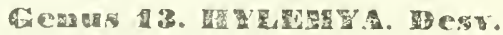

Spec: Typ: Mrsca Straigosa Fall.

AA. Orbita oculorum in mare fascia intermedia maģis vel minus lata, numquam lineari sejunclæ.

O. Palpi subcylindrici vel subclavati numguam spathuliformes.

I. Alo breviores abdomine. - Avdominis segnenta etian prime setis marginalibus el discoidalibus validiusculis instrecta.

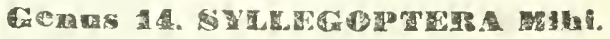

Spec: Typ: Hylemya Ocvpteanata Meig.

IP. Ale longiores abdomine. - Segmenta prima abdominis setis ralidiusculis salıem discoidalibus destiłula. 
Q. Oculi maris superne sutis latiorês spatio frontali intermeảio angusto, ed a medio frontis ad basim anten-narum distincte divergentes non subparalleli.

R. Arista distincte pilosa.

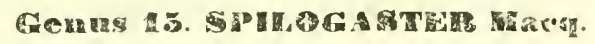

Spec: Typ: Musca Uliginosa Fall.

RR. Arista subnuda.

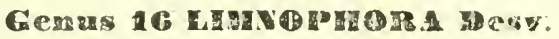

Spec: Typ: Musca Consmus Fall.

QQ. Oculi maris superne circiter lati ut. spatiun fromlals interpositum, et sæpe angustiores fere ut in furmina: in utroque sexu per tolam frontem subparalleli.

S. Arista subnuda ant vix tomentosa.

T. Aristoe articulus secundus nec elongatus nec cum tertio cubitum efficiens. - Vena transversaria prima, contr'x apicem secundæe Iongitudinalis, vel magis extra locata.

\section{Geans 1\%. CAN SIA Meig.}

\section{Spec: Typ: Coenosia Sexiraculata Meig.}

TT. Arista articulus secundus distincle clongatus, prosertim in mare, et cum tertio cubilum efficiens. - Vena prima transversaria satis ante apicen secunda longitudinalis sita.

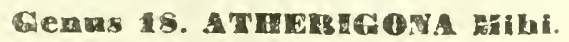

Spee: Typ: Coenoss Yania Meig. 
$9 \delta$

S5. Arista subplumata, ant magis vel minus sed distincle pilosa.

1. Squamce inferm parm ultra superas productæ. - V'enter maris appendicibus validissimis armalus. - Arista hreviter sed manifeste pilosula.

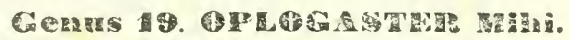

Spec: Typ: Musca Moldicula Fall.

LU. Squance infere calipterorum satis productæe uttra superas. - Venter maris appendicibus vålidis destitulus. - Arista plumata aut subplumata.

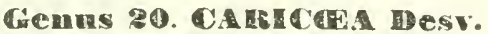

Spec: Typ: Antromya Tugrika Meig.

00. Palpi in spathularin dilatato-compressi.

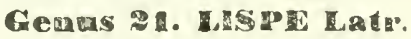

Spec: Typ: Musca Tentaculata Degeel.

Stirps XVHI. SCATOPHAGINA Rndn.

Scatonyzide Latr. Macq: Bigol. - scistonizides Fall. Zett. Westw. - pazourdz Dest.

A. Pulpi iu spathulam dilatato-compressi.

B. Yenalongitudinalis tertia satis ante apicem quinte mar- 
grinem alarum attingens. - Tarsi postici auticills basali non dilatato-compresso - Femora hand valde incrassata.

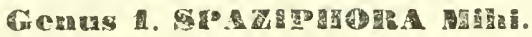

Spec: Typ: Cordylura IIvdroaryzixa Fall.

BB. Vena tertia longitudinalis marginem alarum attingens contra apicein quintæ - Tarsi postici articulo basali dilatato-compresso - Femora quatuor postica in mare satis incrassata, duo ultima crassissima.

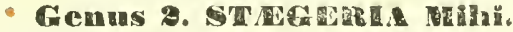

\section{Spec: Typ: Cordylura Kuntzer Zelt.}

A. Palpi vel filiformes vel clavati vel sub-fusiformes, sed in spathulam distinctam non dilatato-compressi.

C. Arista articulus secundus Iongiusculus et cum tertio præsertim in mare cubitum efficiens.

1). Abdomen in mare subtus valde incurvatum: in utroque sexu pilosum sed setis validiusculis destitutum.

\section{Gemus 3. Hyopiva Desv.}

Spec: Typ: $\left\{\begin{array}{l}\text { Musca Mropina Fall. } \\ \text { Myopina Reflexa Desv. }\end{array}\right.$

IOD. Abdomen in mare subtus haud valde incurvatum: in utrogue sexu macrochetis aliquibus praditum.

L. Arista nuda - Tibice intus nec barbatæ nec distincte ciliatæ.

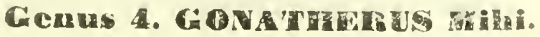

Spec: Typ: Scatomyza Planicers Fall. 
EE. Arista articulo tertio distincte piloso - Tibice anticic et intermediæ distincte ciliatæ, posticæ intus barbatie.

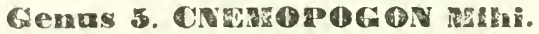

Spac: Typ: Cordylura Apicalis Meig.

CC. Ariste articulus secundus non distincte elongatus, el si aliquando minus brevis, tunc cum tertio cubitum non efficiens.

F. Palpi seta apicali longa et flexu instructi, ab aliis setis, si adsunt distincta.

G. Arista nuda.

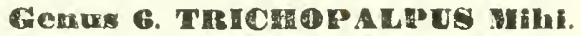

Spec: Typ: Corduqura Fraterna Meig. GG. Arista plumata vel distincte pilosa.

H. Antenne elongatre usque ad epistomium, quarum articulus terlius satis Iongior præcedente.

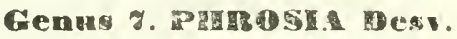

Spec: Typ: Cordylura Alzilabris Meig.

HII. Antenno breves et distantes ab epistomio, quarum al-ticulus tertius parum au: non longior pracedente. Genas 5. C C

Spec: Typ: Cordylura Azbipes Fall.

FF. Palpi seta apicali ab aliis distincia non instructi.

1. Femora ut tibia nedum soticorum spinis validis et longisintus armata. 
k. Arista distincte pilosa. - Tibice intermenaz et postica setis ralidiusculis nonnullis instructx.

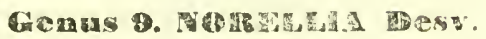

Spec: Typ: Cordulura Stratat Pleig.

hk. Arista nuda. - Tibice quatuor posticat setis validiusculis destitutæ.

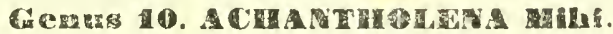

Spec: Typ: (nova) Achantholexa Maculipenis Mihi. II. P'edes antici tibiis et femoribus spinis longis et validis non armatis.

L. Antemne articulo tertio non lenticulari sed oblongo-ovato vel clongato.

H. Aldomen maris præter uncos apicales, appendicibus satis porreclis præditum.

X. Arista subnula. - Spinula costalis non manifesta.

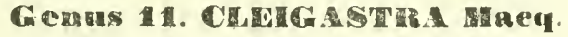

Spec: Typ: Cordyura Nigrata Fall.

NN. Arista distincle pilosula. - Spina costalis alarum distincla.

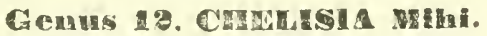

Spec: Typ: Connosia Monilis Meig.

MM. Abdomen inferne appendicibus valile porrectis non pritditum, exceptis alipuando uncis validis apicalibus. O. Vena analis brevissima. - Antenne erectre.

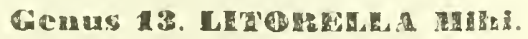

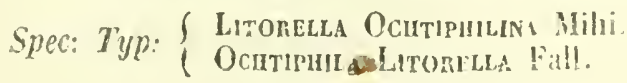


no. Vent analis producta usque as marginem postictun datrum. - Anternce contra facien inc'inate.

P. Arissa nuda vel subnuda.

(1. Tibice antica setis et pilis longis extrineecus destititia. - Oculi maris in medio frontis paulo approximati, sell lion omnino paraileli.

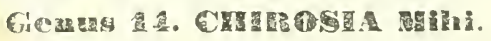

Spec: Typ: Aricla Albitansis Zell.

QQ. Tibice antice setis vel pilis longis extrinsecus prieditix. - Oculi utriusque sexus in fronte omnino paralleli. Gerars

\section{Spec: T'yp: Scatophaga Ixcusa Macq.}

Pr. Arista plumata vel distincte pilosa.

R. Costa alarum spinulis validiusculis a ciliis ordinatiis llistinclis non serrata.

S. Pedes femorilus et tibiis intus barbatis, vel pilis crebris ciliatis. - Spinula costalis indistincta. Genus 16. yCATOPITA Ngn.

Spec: Typ: Musca Stercoraria Lin.

SS. Pedes nec intus barbati nec pilis erebris ciliati serl tantum setosi. - Spinula costalis alarum distincta.

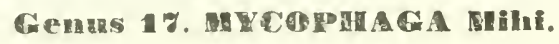

Spec: Typ: Musca Fungorum Degeer.

RR. Costa alarum spinulis validiusculis serrata, a ciliis brevibus ordinariis distinctis.

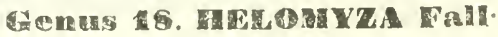

Spec: Typ. Helomyza Ruga Fall. 
LL. Antenne articulo terlio lenticulari.

T. Alce costa spinuloso-serrata; spimulis a ciiis on thitrii. satis distinctis.

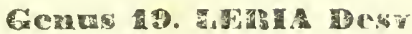

Spec: Typ: Musca Sernata Lin.

Ti. Alie costa non spinuloso-seriata.

I. Epistomizm setis saltem duabus validioribus et longris ab aliis distinctis instructum. - Abdomen maris unci. apicalibus ralidissimis destitutum.

1. Epistomium haid distincte porrectum buccula $\left({ }^{*}\right)$ non manifesta. - Arista ad basim articuli tertii inserta.

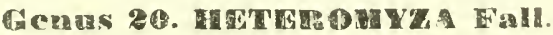

Spec: Typ: Heteronyza Atricornis Meig.

W. Epistomium porrectum, buccula magis vel minus distincta. $=$ Arista in medio dorsi articuli tertii inserta.

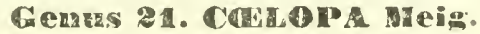

Spec: Typ: Coelopa Fucorum Walb. Zell. UU. Epistomium setis brevibns sub-æqualibus instructum.

(1) La buccula è quella sporgenza in forma di labbro, che trovasi in dirersi ditteri sollo l'orlo superiore dell' epistomio. 
(1)

- Abdomen maris uncis apicalibus validissimis stil veutre flexis.

6 c

Spec: Typ: Orvgara Luctuosa Meig.

STIR. XIX. SC!OHYZINA Rndn.

sciomyzides. Fall. Zetl. - volicocera Latr. Hacy:

- palonyde Desv. - iaclomyzides Westw. - tetiNOCERID Bigot.

A. Alarum vena azalis usque ad marginem posticum producta, aut part!m ante.

B. Antennoe articulo tertio lenticulari.

C. Epistomium baccula porrecta non instructum - Antenner in apice frontis et non sub productione frontali insertx.

Cenus 1. ACTOR Meí.

Spec: Typ: Actors CEstuum Meig.

CC. Epistomium buccula producta instructum. - Antemme sub productione frontali insertæ.

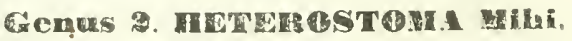

Spec: Typ: Heteronyza Buccata Fall. 
BB. Artennce articulo tertio elonga!o vel conico vel orato sed numquam lenticulari.

Vena longitudinalis secunda valde producta ultra transversam interiorem, et parum ante exteriorem.

\section{Genus 3. DRYONYRA Hall.}

Spec: Typ: Musca Flaveola Fabr.

DD. Vena longitudinalis secunda non aut vix producta ultra transversam interiorem.

E. Areola analis postice elongato-acuminata. - Vence lowgitudinales quarta el quinta versus apicem satis convergentes.

\section{Genus 4. LUCINA Metm.}

Spec: Typ: Lucina Fasciata Meig.

E: Areola analis brevis et non acuminata - Vence longitudinales quarta et quinta non aut vix ad apicem con vergentes.

F. Antennarum articulus secundus distincte long:or tertio, et si raro sub-æunalis tunc arista subnuda aut pubescens tantum.

G. Antenne capite longiores, articulus earum secusdus subcylindricus aut saltem distincte angustior tertio.

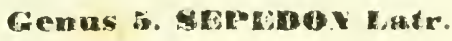

Spec: Typ: Bacca Spheger Fabr

6G. Antenne capite breviores, et si subrequales, tunc articulus earum secundus latitudine tertii et distincte com. pressus. 
d. Vena transucraria secunda in formam $S$ nlexa. - drisk: subnuda.

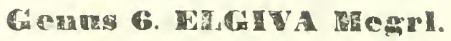

\section{Spec: Tyjp: Musca Cugullatia Lin.}

HH. Tena transversaria secunda vel incurvata tantum, vel. parum sinnosa; si raro satis flexa tunc arista plumata. I. Antonnes ad apisem non penicillate.

G

Spec: Typ: Scatopiaga Rufifrons Fabr.

11. Antenne at apicem penicillatæ.

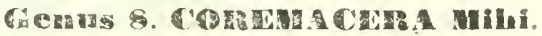

Spec: Typ: Scatopilaga Margixata.

HF. Antennarum articulus secundus te:tio brevior, et si rart: sub-iequalis aut yix longior tunc arista plumata.

K. Antennarum articulus secundtis tertio sub-æqualis ant parum brevior, at smmmun et raro duplo brevior.

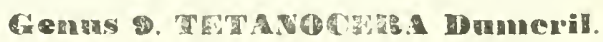

\section{Spec: Typ: Scatophaca Cneropiylli Fabr.}

KK. Antennarum ar'iculus secundus uriplo saltem brevior tertio.

L. Arista superne et inferne snio-reque pilosa vel tomentosa, nisi subnuda.

H. Tibia intermediæ el posticæ setis longiusculis extrinse- 
cus præditæ saltem in parte apicali.

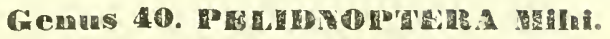

Spec: Typ: Sciomyza Nigripennis Fall.

MIV. Tïbia intermediæe et posticæ setis validiusculis cxtrinsecus destitutæ.

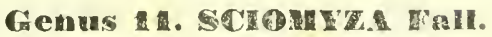

\section{Spec: Typ: Scrouyza Cinerella Fall.}

LL. Arista superne tantum pilis longis instructa.

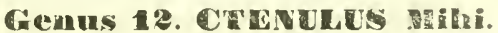

Spec: Typ: Opouyza Pectoralis Stoger.

(In litteris).

AA. Tena analis brevissima sell, satis longe à margine poslico alarum sistens.

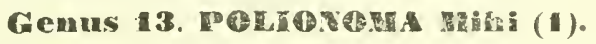

Spec: Typ: a generibus varis, sed presertim Laixania Palloptera Saprouyza elc.

1) Nei generi PoLionous e Scloxnz.s non ho creduso si polessorn stabilire altri generi, fondati soltanio sulle differenze di lunghezza del terzo articolo autennale e dei peli dell' arista, perché questi caralteri nelle specie diverse passano dal piu al meno per grabi ingensibili restando costanti totalmente oli altri carditeri di somig'idaza telis ali dei piedi del sapo ecc. 
108

Stimps XX. ORTALIOINA Rndn.

ortalues Fall. Zelt. Westw. Walk. - carpomzze Latr. - Mrouina Desy. - ortalides Maeq: - trpifritud Latr. Bigot. - acipuore Desy.

A. Venta prima longitudinalis integra et perfecta usque ad costalem.

B. Vena longitudinalis secunda in parte apicali tantum, of non tota superne ciliata.

C. Facics stibnerpendicularis non retro inclinata nec horizontalis.

D. Antenna articulo tertio ad apicem acuminatæ.

E. Aristu pihsa. - Vence longitudinales quarta el quinta ad apicem non distincte convergentes.

\section{Genas 1. Matudith Desv.}

Spec: Typ: Ortalis Crassipennis Fall.

EE. Arisia subnula - Vence longitudinales quarta et quinla ad apicem distincte convergentes.

\section{Cerars 8. Whágla Denv.}

Spec: Typ: Ortalis Marmorea Meig.

DD. Antenne articulo tertio non acuminato.

F: Ancennce articulo tertio breviter ovato.

6. Aniennarum articulus tertius non aut parum longior pracedente - Vena longitudinalis secunda satis producta ultra transversam intermediam.

Genns 3. OhTahis Fall.

Spec: Typ: Ortalis ornata Meig. 
GG. Anternarum articulus terlius bilongior circiter precedente - Vena longitudinalis secunda contra et no! ultra transversam, intermediam, costalem attingens.

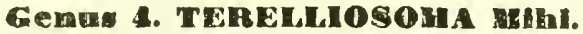

Spec: Typ: Terella Heayngu Macq. (In litteris)

FF. Antennce articulo tertio satis longo, aut saltem elongato sub-ovato.

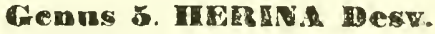

Spec: Typ: Ontalis Nigrina Mirig. :

CC. Facies valde inclinata, vel sub-horizontalis.

H. Antenna satis elongatæ, ariculo tertio subulato-lanceolato.

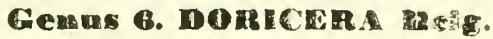

Spec: Typ: Dorigera Graminis Meig.

HH. Antennce breves, articulo tertio breviter ovato vel subrolundato.

1. Intennoe ad basim productione frontali tectæ.

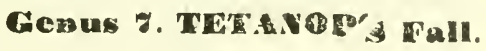

Spec: Typ: Ortals Necuros, Heig.

II. Anienna omnino detecte etiam bas, i.

Genu, 8. OTrTes Latr.

Spec: Typ: Otıтes Nubica Mihi. (nova)

RB. Vena longitudinalis secunda superne tota ciliata.

Genns 8. Phatygtoma catr.

Spec: Typ: Dictia Unaranux Fabr. 
A.t. Vena longitudinalis prima ad apicem interrupta vel in callositate diffusa.

k. Antennce non elongatæ ultra epistomium.

L. Proboscis ad medium cubitata et retro flexa, ant salicm labio apicali retro converso.

M. Areola analis angnlo apicali postico phus minusve sed manifeste acuminato-producto.

\section{Genus 10. OXINA Desv.}

Spec: Typ: Musca Parietina Lin.

Mil. Areola analis angulo apicali postico nec acuminato nec producto.

N. Vena longitudinalis quinta costalem attingens ante apicem alarum, seu ad marginem anticum producta.

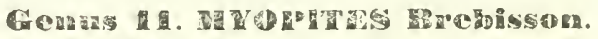

\section{Spec: Typ: Myopites BLotu Breb.}

NX. Vena longitulinalis quinta costalem attingens pust sen sub apicem alarum, in margine postico.

0. Antonmœ non distantes - Facies in medio non gibbosa.

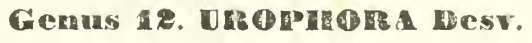

\section{Spec: Tyjp: Musca Solstrtralis Lin}

00. Antemna distantes - Facics in medio valde elevatogibbosa.

\section{Genus 13. GONGEOSAUM Mihi.}

Spec: Typ: Tripeta Wieduand Meig.

LL. Proboscis ad medium non cubitata nec labio apicali longiusculo retro flexo. 
P. Scuiellum setis validioribus duabus vể quatwor, sì rarı setis pluribus instructum, lunc femora antica subten non crebre ciliata, sed setulis aliquibus tantum prædita.

Q. Areola analis apice convexo non concavo, el angulo apicali infero nec acuminato nec elongato. - Venu longitudinalis tertia in costali sub-ærque distans a secunda et a quarta.

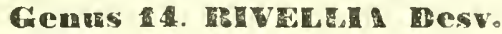

Spec: Typ: Teprritis Singenesie Fabr.

QQ. Areola analis apice concavo, et angulo infero apicali magis vel minus accuminato e! elongato, et si raro non manifeste talis, tunc vena terlia longitudinalis magis proxima quartæ quam tertiæ in costali.

R. Fence transversarice intermedice sub reque distantes ab exteriori et a basalibus, aut saltem distantia a basalibus non duplex distantia ab exterior:.

S. Vena longitudinalis secunda tantum stiperne Ciliata. T. Antennce forma ordinaria et in cavo frentis non localæ.

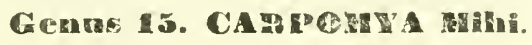

Spec: Typ: Musca Aвсти Degeer.

TT. Anfennarum articulus secundes in formina manifeste product!s, in mare cornu longo preditus, in utroque sexu antennæ in cavo frontis locatæ

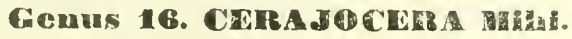

Spec: Typ: Musca Cornuta Fabr.

SS. Vence longitudinales secunda et quar:a superne ciliatx. 
U. T'ena longiludinalis quarta incurvala et postice flexa. Epistomium macrocletis lateralibus instructum.

\section{Giems 1\%. CHETOSTOMA MHL.}

Spec: Typ: (nova) Caetostora Curvinervis Mihi.

UU. Epistominm setis validis destitutum. - Vena longitudinalis quarta nec manifeste incurvala nec retro flexa. Y. Scutellum setis quatuor præditum, - Femora quatuor postica inferne breviter sed distincte omnino ciliata.

\section{Conus 18. EPIDESMA Mihi.}

Spec: Typ: Taphritis Cognata Wiedm.

VV. Scutllum setis duabus tantum instructum - Femora non ciliata.

\section{Gemus 10. MTOLEJA Mihi.}

Spec: Typ: Tepuritis Lugida Fall.

RiR. Vena transversa intermedia saltem duplo distans a basalibus, quam ab exteriori transyersaria.

X. Oris apertura tota, infera, seu in facie non distinete ascendens. - (Ala maculata).

Z. Antennce articulo tertio dorso recto, et apice non acurainato.

Y. Scutellum setis qualtuor præditum.

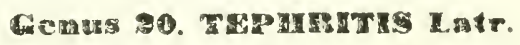

Spec: Typ: Musca Leontodontrs Degeer.

YY. Scutellum setis duabus tantum instructum. 
2. Proboscis non producta; Palpi nec Tongi nec spathuliformes et proboscidem non tegentes.

\section{Genus 21. AClura Desv.}

Spec: Typ: Acilra Femoralis Desv.

\&2. Proboscis paulo producta: Palpi longiusculi spathuliformes et proboscidi superpositi.

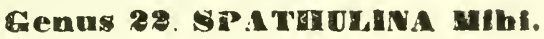

Spec: Typ: Spatuulima Sigulla Mihi (nova)

ZZ. Antennce articulo tertio dorso magis vel minus concavo, et apice plus minusve distincte acuminato.

a. Scutellum setis duabus tantum instructum.

\section{Genus 23. DITHA YCA Milh.}

Spec: Typ: Tripeta Gutrularis Meig.

as. Scutellum setis saltem quatuor instructum.

b. Scutellum setis quatuor majoribus et aliis minoribus præditum.

\section{Genus 24. OrLochets whi.}

Spec: Typ: Tephritis Pupillata Eall.

ht. Scutellum setis quatuor tantum instructum.

- Veno longitudinales secunda el quarta superne ciliatio.

Genus 25. ACIvI. Desv.

Spec: Typ: Tepuritis Cogniculata Fall. re. Vena longitudinalis secunda tantum superne ciliata.

Genus 26. OREMLIA Dew.

Spec: Typ: Tepuritis Radiata Fabr. 


\section{4}

XX. Apertura oralis in facie distincte ascendens. (Ala int maculate).

\section{Genus 37. Tathedula Desv.}

Spec: Typ: Musca Serratule Lin.

PP. Scutellum setis sex instructum. - Femora antica inferne setis longis plurimis et crebris ciliala.

Gorans 28. Cenatisis mac. Leay.

Spec: Typ: Ceratitis Hispanica Breme.

KK. Antennoe ultra epistomium elongatæ.

Gemus 29. DACCS Meig.

Spec: Typ: Musca Oleæ Rossi.

Grips XXI. TANIPEZINA Rndn.

leptopodte Latr. Macq. - nerhades Westw. uldini (partim) Macq. - oponyzide Fall. Zetl. тrendionyde Desv. - Sepsidex Fall. Macq: - Sepsides Walk. - psieldes p. Walk. - Leptopoditidex Bigut.

A. Epristomium omnino nudum.

13. Femora antica saltem inferne setis vel setulis ciliata.

C. Areola analis ad apicem angulo infero non ant vix producto et acuminato.

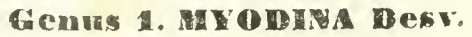

Spec: Typ: Mrsca Vibrans Lin. 
CC. Areola analis angulo infero apicali satis elongato-acuminato.

D. Antenne in cavo faciei non locatæ. - V'ence longitudinales quarta el quinta satis convergentes versus apicent, et ibi valce approximatæ.

\section{Genus 2. URIDIA Meig.}

Spec: Typ: Tepuritis Deruandata Meig.

DD. Antennce in cavo faciei localæ. - Venæe longitudinales quarta et quinta quamvis convergentes, ad apicem parum approximatæ.

\section{Genus 3. TIma Deig.}

Spec: Typ: Uidia Erytiroputhalita Meig.

BB. Femrora antica nula vel subnuda.

E. Fence iongitudinales quarta et quinta non distincte couvergentes.

F. Femora postica satis incrassata et subtus spinuloso-serrata.

\section{Genus 4. HSSA Heig.}

Spec: Typ: Cumlza Loxocerina Fall.

fF. Femora postica nec incrassata nec subtus spinulosa.

Genus 5. Corllatha neig.

Spec: Typ: Cepualia Nigripes Meig.

WE. Tena longitudinales quarta et quinta distincle conscgentes versus apicem.

G. Palpi apparentes et distincte dilatati.

Genus 6. TANITPRa rall.

Spec: Typ: Tanipeza Longimana Fall. 
$\$ 16$

GG. Palpi forma ordinaria, vel parum apparentes.

H. Arista nuda vel subnuda.

I. Areola anteanalis completa.

Genug 7. TANRPATR Mihi.

Gemur Raivieria Rojo. (ollim.)

Spec: Typ: Calobata Calceata Fall.

II. Areola anteanalis nulla.

Gemns 8. Wichopeza heig.

Spec: Typ: Migropeza Corrigiolata Meig.

HH. Arista plumosula vel distincte pilosa.

Genus o. Calobata Melg.

Spee: Typ: Musca Cibanta Lin:

AA. Epistomium paulo setulosum vel pilosulum.

K. Areola anteanalis completa seu apice non aperta (scutellum setis duabus tantum.)

L. Femora anlica in mare subtus denticulata vel spinulosa. M. Abdomen penicillis caudalibus nullis. ( $\delta$ ll in utreque sexu puncto nigro notatæ).

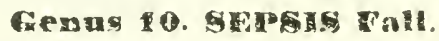

Spec: Typ: Tepurtis Puxctur Fabr.

MM. Abdomen penicillis caudalibus in mare prsediturn (Alæ in utroque sexu impunctalæ).

․ Tarsi in utroque sexu simplices.

6). Tibret anticx maris extringecus nec incisie nec appendice 
præditæ - Penicilli caudaies parvi et non erecti.

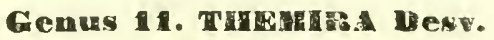

Spec: Typ: Musca Putris Lin.

00. Tibioe antica maris exIrinsecus incisæ et appendice prixditæ - Penicilli caudales longi erecti et subplumati.

\section{Gemus 12 mamidaxa mith.}

Spec: Typ: Themara Setosa Dest.

NN. Tarsi intermedii maris dilatati - Fæminæ articuli prisni tarsorum basi compressi - Tibia antice maris bidentatæ.

\section{Genus 13 Evicita Wextw.}

Spec: Typ: Sepsis Anxulupes Meig.

1.L. Femora antica maris inferne nec spinulosa nec dentala. Genus 14 venorod sarq.

Spec: Typ: Sepsis Cyundrica Fabr.

KK. Areola anteanalis incompleta, seu apice aperto. (ScutelIun setis quatuor).

\section{Cenus 15 Salthelu benv.}

Spec: Typ: Pyophila Pecroralls Fall. 
118

STrns XXll LONCHEINA Fuln.

Lauxanide (part.) Macq. Bigot. - Lauxaxides Walk. - vlide p. Bigot. - oporysydes p. Fal!. Zett. - geonyzides p. Fall. Zett. Westw. Walk. psilides p. Walk. - sarrouyzides p. Westw. Haliı.

- plophldax Macq. Bigot. - propuylides IVeslw.

A. Facies in merlio valde excavala - Antemno in cavo faciei localæ.

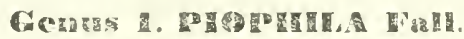

Spec: Typ: Musca Casel Lin.

A. Facies haul valde excavata. - Antenne in cavo faciui non locatæ.

13. Arista superne pilis longiusculis ciliata, inferne breviter vel brevissime pilosula.

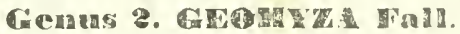

Spec: Typ: Geouys Tnipunctata Fall.

BB. Arista subnuda vel superne et inferne pari modo pubescens.

C. Antenua erectæ, articulo tertio magis vel minus distincte al apicem acuminalo.

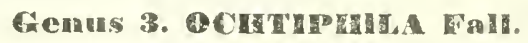

Spec: Typ: Ochtiphila Polistrgin Meig.

C. Antenna non erectw; articulo tertio numquan apice acuminato nec sub-acuminato. 
5. Vence longitudinales prima et secunda ad apicem tantum paulo distinctæ et divergentes, a medio al basim in unica confusæ.

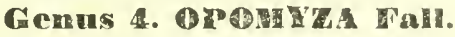

Spec: Typ: Musca Florum Fabr.

DD. Vence longitudinales prima et secunda a basi ad apicem distinctæ, etiamsi in medio distincte approximatæ. E. Arista sub-apicalis. - Vena longitudinales prima et secunda subparallelæ usque ad apicem, non divergentes.

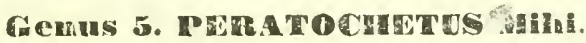

Spec: Typ: (nova) Penotachetus Lutescens Mihi.

EL. Arisla in medio articuli tertii non ad apicem inserta. -

Vence longitudinales prima et secunda ad apicenı magis vel minus divergentes.

F. Antennœ articnlo tertio numquam perfecte lenticulari.

G. Oculi nudi. - Ancennarum articulus secundus satis uninor. tertio.

II. Antennce breves, ab epistomio satis distantes: articula tertio breviter ovato.

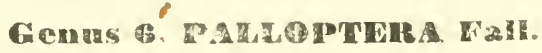

Spec: Typ: Musga Umbelatarum Fabr.

HII. Antennce productæe circiter usque ad epistomium: articulo tertio satis elongato.

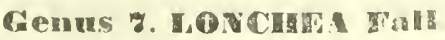

Spec: Typ: Musca Cúorea Fabr. 
GG. Oculi pilosi. - Antennce articuils secundo el tertio sub-æqualibus.

\section{Genus 8. DA810P, Mihi.}

$$
\text { Spec: Typ: }\left\{\begin{array}{l}
\text { Dasiops Loncheus Mihi. } \\
\text { Lonchea Dasiops Mgn. }
\end{array}\right.
$$

FF. Antenno articulo tertio perfecte circulari, seulenticulari.

Genus D. LevCOPA Meig.

Spec: Typ: Leucopis Griseola Heig.

STIRPS XXIII. AGROMYZINA Rndn.

agromyzioes Fall. Zell. - geomyzides (part) Fall. Zett. - ueteromyzioes (part:) Fall. Macq. Zett. PHytomyzides p. Walk. Bigot.

A. Alo margine antico contra venam primam longitudinalem profunde secta.

\section{Genus 1. Muchis Meig.}

Spec: Typ: Milicr: Speclosa Meig.

AA. Ala margine antico non profunde secto.

:B. Epistomium distincte pilosum, setulis nonnullis vibrissinis.

- Vena longitulinalis prima basi duplex ad apicen unica. 
6. Fonora postica distincle incrassata.

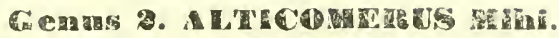

Spec: Typ: (nova) Atucom: Trunotatus Miti.

6C. Femora postica non manifeste incrassati.

1). Femora antica distincte incrassata.

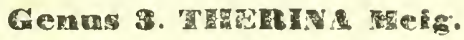

Spec: Typ: Theriwa Feroralis Meig.

DD Femor a antica non incrassata.

E. Vena costalis contra apicem lertiæ longitudinalis sistens.

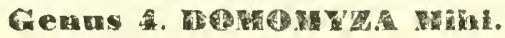

Spec: Typ: (nova) Donomyza Cricts Mihi.

Wh. Venu castalis contra apicem quartæ longitudinalis sistens.

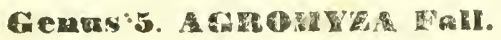

Spec: Typ: Agromyza EneIventris Fall.

BB. Epistomitm subnudum, setis ribrissinis nullis. - Tena prima longitudinalis duplex usgacic ad anicem, seu venis duabus satis approxinatis el in costali conjunctis conprosita.

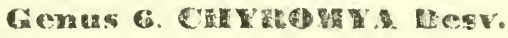

Spec: Typ: Cuynorya Fenestrarum Dest, 
STIKPS XXIV. GHLIZINA Rndn.

psiloryes part. Macq. Bigot. - psiludes Walk. Loxocemise Macq. Bigot. Westw. - palomyde p. Desv. - cordinurise. p. Mucq. - scatomyzide p. Lair. Full. - oporyzids p. Fall. Zett.

A. Areolo andis et anteanalis xque vel sub-aque elongata.

- Vena longitudinalis quinta post transversam exieriorem magis vel minus incurvata.

B. Antennce satis breviores capite.

C. Femora postica, prasertim in mare, incrassata et incurvata.

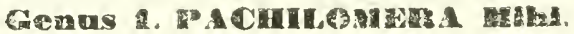

Spec: Typ: Psira Lefebvaei Zelt.

CC. Femora postica in utrogne sexu nec inflata nec incurvatso

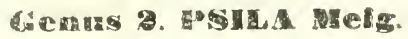

Spec: Typ: Psila Roste Meig.

BB. Antenne satis longiores capite.

D. Antenna articulo tertio immodice elongato, primis duobus urevibus.

\section{Geans 3. LOXCCLI A Fhr.}

Spec: Typ: Loxocera Distincta Zett.

DD. Antennce arliculo secundo elongato, tertio longiore.

Gemus 4. PLATYSTILA Macq.

Spec: Typ: Loxocera Hoffmanseggu Meig. 
A. Areola antranalis lislincte longior anale. - Vena quinea longitudinalis post transversan exteriorem recta vel sub-recta.

ـ. Arista crassa el brevibus el densissinis pilis subplumata.

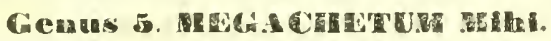

Spec: Typ: Cumba Arriseta Meig.

EE. Arista non crassa, pilis exilibus sed non crebris itim siructa.

Genus 6. Crimbat Helg.

Spec: Typ: Culuza Vitrata Meig.

STIRPS XXV. COPROMYZIYA Rndn.

Spherocera Latr. - spuerocerids Macq. Bigot. Borbonides Westw. Walk. - copronyzine Zett. tuyreoploride Macq. Bigot. - Heteronyzide p. Fall. putrellidee Desv.

A. Aloe distincte.

B. Befetalarsus posticus noa incrassato abbreviatus.

Cenus ThYREOPHore Esitr.

Spec: Typ: Musca Furcata Fabr.

BB. Metatarsus posticus incrassato-abbreviatus. 
C. Areole analis et anteanalis distincte, aut saltem anteam nalis completa - V'ena iongitudinalis quarta perfecta.

D. Basis alarum non ciliata.

E. Vena quinla longitudinalis ad marginem alæ prolucta.

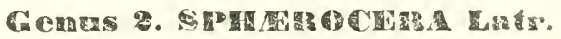

Spec: Typ: Musca Subsultais Lin.

EE. Vena longiludinalis quinia ad marginem usque alurure non producta.

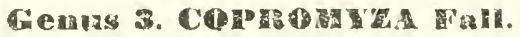

Spec: Typ: Borborus Furtarivs Mejig.

DD. Basis alarum ciliata.

1. Vence transversariæe dux distinctæ.

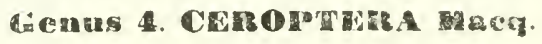

Spec: Typ: Borbonus Rufitarsis Meig.

FE. Tena transtersaria unica distincta.

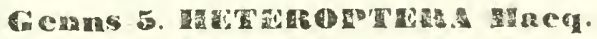

Spec: Typ: Copnoniza Pusilla Fall.

CC. Areolce arales aut anbæ indistinctie aut anteanalis nulta

- Vena longitudinalis quarta spuria aut incompleta. G. Ala longiludine ordinaria - Vena transversa exterior Inanifesta.

\section{Gemas 6. Homegrivi Macq.}

Spec: Typ: Lirosina Silvatica Meig.

GG. Ala valde abbrevialæ. -- Vena transversa exterior nulla .

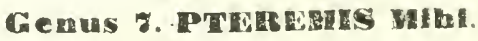

Spec: Typ: Luxosixa Nivalis Halid. 
AA. Ales omnino nullix:

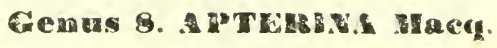

Spec: Typ: Borborus Pedestris ingn.

Straps XXVI. CLOROPINA Rndn.

oscinibes part. Fall. Zelt. Halid. Walk - heteromyzides p. Macq. - oscinide Biget.

A. Alce brevissimæ vel subnullæ saltem in foenina.

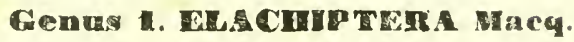

Spec: Typ: Crorops Brbvipennis Meig.

AA. Alie compleiæ.

B. Vena costalis saltem usque ad apicem tertiæ longitudinalis crassiuscula.

C. Facies etiamsi inclinata non horizontalis. - Frons haud clongato-sub-acuminata, cujus pars post oculos longior anteriore.

D. Femora postica non satis inerassata.

Genus 2. CHLomors areis.

Spec: Typ: Chlonors Lata Meig. DD. Femora postica distincte incrassata. 
120

l. Veno Longitudinalis teria marginem anticum alarun petens. - Antcrnoe articulo tertio rotundato.

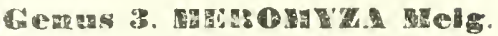

Spec: Typ: Meronyza Variegata Meig.

HE. Yena longitudinalis tertia sub apicem alæ marginem attingens. - Antenne articulo tertio lanceolato.

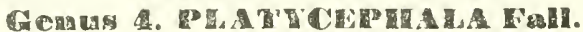

Spec: Typ: Oscinis Planifrons Fabr.

C.C. Facies horizontalis. - Frons distincte elongata cujas pars post oculos brevior anteriore.

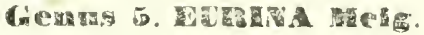

Spec: Typ: Euriva Lurida Meig.

BB. Fena costalis in parte basali tantum crassa, non esque ad apiceu vena iertix longitudinalis.

Gervas 6. CAR M

Spec: Typ:- Camarota Flavitarsis Meig. 
StoRps XXVII. OSCININA Rnetn. (")

oscinides part. Fall. Zett. Halid. Walk. - uetr,-nunyzides p. hacq. - oscimide Rigot.

A. Vena longitudinalis quarta perfecta et distincta.

B. Arista antennarum satis incrassata.

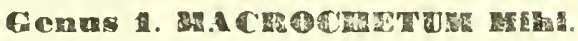

Spec: Typ: Oscinis Connuts Fall.

BB. Arista haud distincte incrassata.

C. Antennarum articulus tertins dente subapicali praditus.

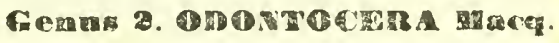

Spec: Typ: Clorops Denticomis Panz.

CC. Antennarum arliculus tertius haud dentatus.

D. Antennce articulo tertio satis lato et subquadrato.

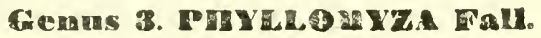

Spec: Typ: Puyllonyza Secra!corsis Fall.

DD. Antennæ articulo tertio latitudine ordinaria, et si paulo latiusculo subcirculari.

E. Proboscis crassiusctla etiamsi cxtrinsecus cubitata et

(1) Forse questa slirpe potevasi congiungere colla pracedenie conservaudo per le due stirpi riunite o l'uno o l'altro dei due nomi distiatini di Oscivisa o Cronopiva; io le divido perché do gran valore atle differenze dell $\Delta$ li e del Lipistomio. 
retro flexa, et quando talis Hnc par's intus flex: brevis.

\section{Geams 1. ACINIS Mat".}

Spec: Tyn: Culorops Varipes Meig.

PR. Proboscis exilis et extrinsecus cubitala, pars retro flexa satis longa.

*. Vena longitudinalis secunda costalem allingens satis longe ab apice alarum. - Vena transversa exterior magis proxima interiori gram margini postico.

G. Vena longitudinalis secunda, in costali magis proxima primæ, quam tertiæ.

Gerns 5. ร10

Spec: Typ: (nova) Siphucuera Brevinenvis Mihi. GG. Vena longitudinalis secunda in costali magis distans a prina quam a tertia.

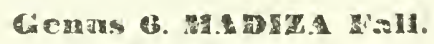

Spec: Typ: Mad: Oscivisa Fall.

FF. Fena longitudinalis secunda costalem attingens ad apicem vel prope apicem alarum. - Venatransversa exterior magis distans ab interiori quam a margine postico.

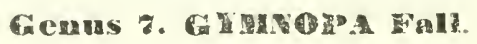

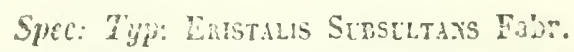

B. Hend loigitudinalis quarta, spuria et vix distingnenda.

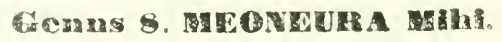

spec: Typ: Agromiza Obscurelda Fall. 
STiRps XXVII. LYHIDRINA Rudn.

nydromyztres Fall. Macq. Westw. Bigot. Walk. hydrellide Desv. - ephydRane Zett. - plophllues p. Macq.

A. Fena costalis contra apicem tertix longitudinalis sistens.

I. Adomen macrochetis ralidis nullis, et appendice longa ventrali destitutum in utroque sexu. - Epistomium subnudum, vel pilis exilibus et brevibus instructum.

C. Vena longitudinalis secunda costalem attingens contra transversam exteriorem. - Vena longitudinal is quartit a transversa exteriori ad apicem spuria.

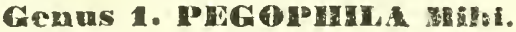

Spec: Typ: (nova) Notipulla Meridionalis. Schembri (in litteris).

CC. I'ena longitudinalis secunda costalem attingens satis nitr.d transversam exteriorem - Vena longitudinalis quarta usque ad apicem perfecta.

\section{Genus 2. NoTIPIHI, Fall.}

Spec: Typ: Notirula Cinerea Fall.

BB. Abdomen maris macrochetis validis et appendice long a rentrali præditum. - Epistomium in utroque sexu setas validiuscnlas prebens.

Gemas 3. Henema han.

Spec: Typ: Notrulla Caudata Eall. 
AA. Vena costalis contra apicem quaria longitudinalis producla.

D. Epistomium setulis vel pilis pendentibus inferne marginatum.

k. Arista nuda. - Tursi articulo ultinı, apicali distincte elongato.

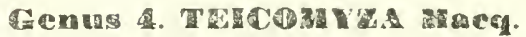

Spec: Typ: Teiconyza Fusca Macq.

EE. Arista pilosa, ciliata, vel pubescens. - Tarsi articulo extremo hand distincte elongato.

\section{Genus 5. Th8}

Spec: Typ: Ephydra Stagnalis Fall.

DD. Epistomium setulis et pilis pendentibus non instructum inferne.

F. Femora anlica non incrassata distincte. - Vence longitudinales tertia et quarta ad apicem non satis approximalæ.

(1) Compreade i Generi Coenta e Napasa di Desvoidi, distiali solamente per la lunghezza diversa dei peli dell' arista, i quali secundo le specie passano gradatamente dal più al meno.

Però se ad alcuno piacesse accettare $i$ due Generi distioli sarebbero cosi caratterizzati.

a. Arista distincte radiata.

\section{Gedus Cervia Degy.}

Spec: Typ: Trubra Palustais Fall.

aa. Arista pubescens vel tomentosa.

Genus Napea Desr.

Spec: Typ: Epgidas Quadata. Fall. 
G. Vena transversa exterior magis vel minus sed distincte ultra apicem primæ longimdinalis sita.

H. Alarum costre segmenum secumum longitudine circiter æquale tertio. - Abclominis segmenta tria cieteris satis latiora.

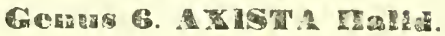

\section{Spec: Typ: Trherias Cerveleiventras Macy.}

HH. Alarum costee segmentum secundum magis vel minus sed distincte longius tertio. - Abdominis segmenta qunat!or vel çuinque sub-ærualia.

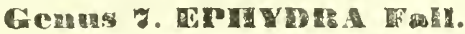

Spe: Typ: Epiydra Cosrctata Fall.

GG. Vena transversa exterior, ante, vel ad summum contra apicem primæ longitudiralis sita, et non extra.

1. Antennce articulo secundo setis validiusculis superis destituto.

\section{Genus 8. HYDREGUA Desy.}

Spec: Typ: Notipulla Griseola Fall.

11. Antennœ articulo secundo setis aliquibus validiusculissuperne instructo.

K. Proboscis bicubitata. - Venter non insectns.

\section{Genus o. Hedcarion Crartis.}

Spec: Typ: Notipulua Albicass Mggn.

KK. Proboscis non bicubitata. - Venter insectus. 
L. Arista subnuta. - Frons seiulis et pilis longiusculis destituta.

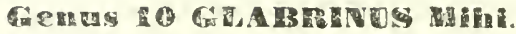

Spec: Typ: (ncva) Glabranus Murorum Mihi.

LL. Arista superne ciliata. - Frons setigera.

A. Vena transversa exterior nlagis proxima interiori, quam apici quartæ lungiludindis.

N. Abdomen segmentis quattor aut quinque sub-æque latis.

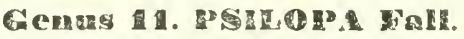

Spec: Typ: Psizopa Nitidere Fall.

NN. Abdomen segmentis tribus intermediis latis, basali of apicali brevissimis.

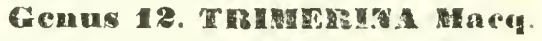

Spec: Typ: Psilopa Madizans Fall.

MM. Vena transversa exterior magis distans ab interiori, quam ab apice quariæ longitudinalis.

Genus 13. BISCOMYRa nes.

Spec: Typ: Psilopa Incurva Fall.

FF. Femora antica distincte incrassata. - Vena longitudinales tertia et quarta ad apicem satis approximatx.

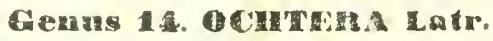

Spec: Typ: Musca Mantis Lin. 
STIRPS XXIX. DROSOPHILINA Rndn.

geonyzies p. Fall. Westr. Zct. Walk. - piopurLIDES p. Macq. Bigot.

A. Arista pilis pancis raris et longiusculis ciliata.

B. Vence longiiudinales tertia et quarta ad apicem distincte. appraximatx. - Alce fractæ.

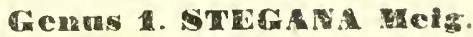

Spec: Typ: Stegaxa Nigra Meig.

BB. Vence longitudinales tertia et quarta ad apicem non distincte approximati. - Alce non fractæ.

C. Vena longitudinalis prima distincte producta ultra priinam transversam.

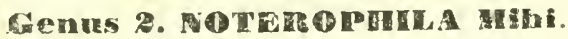

Spec: Typ: Drosopmli Glabra Fall.

CC. Vena longiludinalis prima non producta manifeste ultra primam transversam.

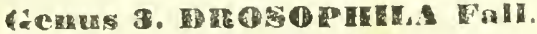

Spec: Typ: Mesca Celararis lin.

AA. Arista subnuda vel pubescens, vel pilis densis subpluinata.

D. Arista non cubitata, articulo secundo non elongato, et $s$ i aliquando minus breve; tunc costa alarum antica spinulosa. 
E. Margo anticus alarkm uon spinulesus.

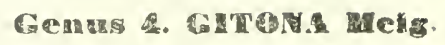

$$
\text { Spec: Typ: Gitona Distygua Meig. }
$$

EE. Alarum margo anlicus spimulosus.

F. Vena transversa interior magis distans ab exterioriquan

a basalibus. - Arista pi!osa vel subplumata.

\section{Genus 5. DIAT'}

Spec: Typ: Diastata Costata Meig.

PF. Vena transversa interior circiter æquidistans ab exterior et a basalibus. - Arisia subnuda aut breviter pubescens.

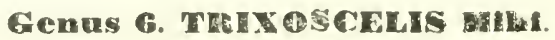

Spec: Typ: Geomyza Obscurella Fall.

DD. Arista cubitata, articulo secundo longiusculo. - Alarum costa antica non spinulosa.

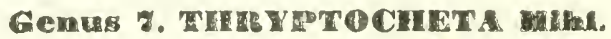

Spee: Typ: Diastata Punctum Meig. 
STIKPS XXX. ASTEJINA Ridn.

Paytonysides p. Fall. Zet. Walk. - hetronohyzids

p. Macer. - AGrouYzide p. Bigot.

A. Arista nuda vel pubescens.

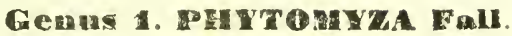

Spec: Typ: Paytohyza Affinis Fall.

1. Arista pilis longis et paucis radiata.

Genus a. ASTEJA Meig.

Spec: Typ: Astesa Concinsa Meig.

SnIRP XXXI METOPININA Rndr.

Synonim: Famil: Phorroorum.

\section{Gemus 1. HETORINA Harq}

Spec: Typ: Puora Galeata Halid. 
139

Stirps XXXY. PHORINA Findn.

Synon: Famil: PuortDarux.

A. Antenna maris artienlo tertio conico, elongato, erecto, et arista apicali.

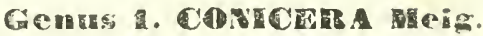

Spec: Typ: Conicera Atra. Meig.

A. Antennce in utroque sexu similes, articulo tertio sulrotundato, vel non erecto, el arista dorsuali.

B. Vena secunda longitudinalis apice non bifido.

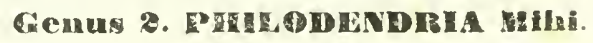

Spec: Typ: Tepuritis Aterrima Fabr.

bB. Vena longitudinalis secunda apice bifido vel furcato.

C. Vence postica semispuriæ tres tantum.

D. Vena semispuria prima oriens in utroque sexu conlra basim furcæ venosæ marginalis. - Palpi maris longissimi, lati, compressi et contra faciem elevati.

\section{Gienus 3. PAr.PInEGA Mhi.}

Spec: Typ: (nova) Palplmega Nivalis Mihi.

Do. Vena semispuria prima oriens in utroque sexu contra medium furcæ venosæ marginalis. Palpi in mare et foemina magnitudine ordinaria aut parum diversa.

Genus 4. TREFHERA Min.

Spec: Typ: (nova) traphleba Hyemalis Mihi. 
1C. V'ence semisprorice posticæ qualuor.

E. Basis alarum marginis antici non ciliata.

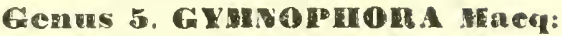

Spec: Typ: Рнora Ancuata Meig.

EE. Ala margine antico ad basim ciliatie.

V. Vena costalis ad basim valde incrassata et clilatata et cum prima longitudinali confusa.

\section{Genus 6. MEgasten wihi.}

Spec: Typ: (nova) Megaseua Crassineuna Mihi.

LF. Vena costalis basi non incrassata et a prima longitudinali distincta.

Genus 7. Here Eatr.

Spec: Typ: Triaeura Rufipes Fall.

Stiars XXXIII LONCOPTERINA Rndn.

Syon: Famil: Loncopteridarur.

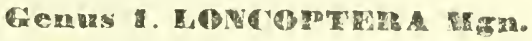

Spec: Typ: Loncoptera Fldutcauda Meig. 
renps XXXIY. OPETIIN Pindn.

Siron: Famil: Platypezidarux.

1. Tarsi postici non diatati - Vena longihudinales yuinta el sexta furcam renosan costiluentes.

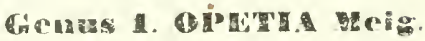

Spec: Typ: OpeTia Nigra Meig.

1A. Tarsi postici manifeste dilatati. - Vene nulbe Inngitudinales furcam venosam constimentes.

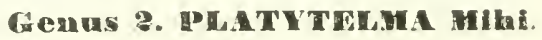

Spec: Typ: Crroma Paluprs Mgn.

STIRPS XXYY. PLATYPEZINA Rndn.

Synov: Famil: Platypezldarum.

A. Vena longinudinalis quarta recta et non appendiculata. Genus 1. Cathoric Fall.

Spec: Typ: Callomya Antenvata Fall. 
B.1. Tena longitudinalis quarta cubitata, cubito appenliculato.

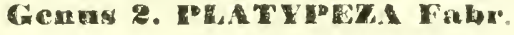

Spec: Tyjp: Platypeza fasciata Fabr.

86

Sul:s XXV. PIPUXCULINA Radn.

Synom. Familiæ Pipunculdoron.

A. Veria longitudinalis quinta completa et usique ad narginem alæ prolucla.

b. Vence aumante longitulinalis cubilus appentiec venosa prærlitus.

C. Antenno articulo tertio reniforme.

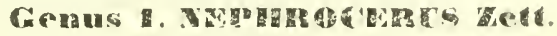

Spec: Typ: Pupencules Scurellatus ildey.

CC. Antenno articulo tertio inferne acuminato sib-lunceolato.

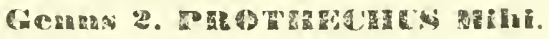

Spec: Typ: Pipuncules Acorus Rall.

BB. Fence longitudinalis quintx cubitus non appendiculatus.

5. Vena longitudinalis prima costalem alluggeus contra transtersam intermediam. - Longitudinalis seeuda in costali producta salis ultra transcersam upsan intop- 
mediam. - Transersaria ista magis proximá interiori quam exteriori.

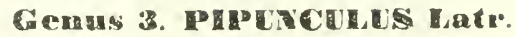

Spec: Typ: Pupracus Romals Meig.

3). Tenue longinulinales prima et secunda costalem attingrentes ante transversam intermediam: ista magin proxima Iransversa exteriori quam interiori.

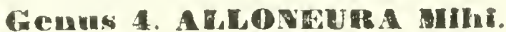

Spec: Typ: Pipurculus Flavipes Meig.

1. Fena longiludinalis quinta contra transversam exteriufem sistens: extrinsecus nulla.

Genus 5. ATELENERA Macq.

Spec: Typ: Cephalops Spurius Fall.

STIRPS IXXVII. DOLICOPINA Rudn.

Synonim: Famil: Doucopidorux.

A. Fena prima longitudinalis costalem attingens circiter contra transerersam ordinariam, et si partm ante tunc arista subapicalis.

B. Antemne in utroque sexu in parte infera capitis insertie. 
- Oculi maris in fronte contigui.

\section{Genus 1. DxapHORES Mgh.}

Spec: Typ: Diapiozus Oculatus Mleig.

3i. Antennce utriusque sexus in parte supera capitis inserta. - Oculi maris et fominæ non contigui in fronte.

\section{Genes ż. ARGYRA Macq.}

Spec: Typ: Dolicopus Diapianus Fabr.

A. Tena prima longitudinalis costalem attingens satis ant valde ante transversam ordinariam, et si minus anie tunc arista dorsualis distincte, non sub-apicalis.

C. Vena trausversa ordinaria satis proxima margini postico alarum, seu longior distantia ab apice quintæ longitudinalis, aut ad summum æquidistans et tunc verio longitudinalis quarta sub apicem alæ aut in apice ipso sed non manifeste ante apicem costalem attingens. F. Fomora antica subtus distincte spininloca.

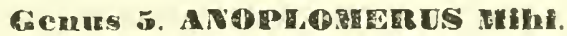

Spec: Typ: Ifrdropuonus Regres Fabr.

EE. Femora antica non spinulosa.

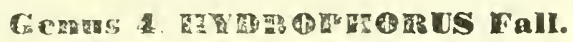

Spec: Typ: IIydnophorts Notatus Meig.

C.. Tena transversa ordinaria satis distans a margine postico alartun aut saltem brevior distantia ab apice quinto longitudinalis, et si raro fere xutue longa, tnnc: rena quarta longitudinalis costalcm attingens manifeste ante apicem alix. 
142

G. Vena longitudinalis quarta extrinsecus angulata, angulo appendice longa prædito. - Pedes spinulis trevibus. - Organa copulatoria macis laminis latis non insiructa.

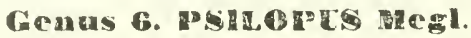

\section{Spec: Typ: Dolicorics Phatiptenus Fabr.}

SG. Fena longitudinalis quarta si raro cubitata et simul appendiculata tunc tibio omnes spinis validis instructæ, et organa copulatoria maris laminis latis prædita.

11. Fena quarta longitudinalis post apicem vel in apice alarum sed uon ante, costali producta. - Amus marium laminis latis non instructus.

1. Tena analis longa, usque vel fere ad marginem alarum posticum ducta. - Vena longitudinalis quarta extrinsecus magis vel uninus flexa.

\section{Genus \%. NUgagova mini.}

Spec: Typ: $\left\{\begin{array}{l}\text { Musca Quadrifasciata Lin. } \\ \text { Porphiliops Quadr: Mgn. }\end{array}\right.$

11. Tena analis brevis vel brevissima, et magis vel minus longe a margine postico alarum sistens. - Tena longitudinalis quarta recta vel subrecta.

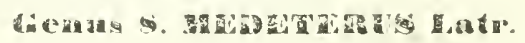

Spec: Typ: Medeterus Curvipes Meig.

HII. Yena bonginudinalis quarta costalem attigens magis vel 
unimus ante apicem alarum, raro satis prope apicern. ef tune organa copulatoria maris laminis latis instructa.

1. Antenne maris a ticulc ul timo erecto acuminato elongato; secundo brevissimo: - Fomina articulo tertio satis parvo, secundo lato. - Arista in mare apicalis, articulo penultimo maxime elongato, ultimo brevissimo cum præcedente spathulam efficiens; fœminæ articulo ullimo longissinuo ut in aliis generibus et in dorso articuli tertis antennaruu inserta.

\section{Gents 9. MARTA}

Spec: Typ: Haltericerus Impar Rndu.

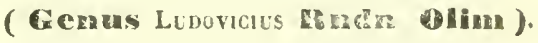

¿L. Anema et Arista utriusque sexus luma ordinaria el sub-æqu!a!es.

3. Arista arliculo penultino distincte elingato.

N. Tena transversa ordinaria satis distans a margine postico alarum, aut saltem distinctc breviu! distantia ab apice quartæ longitudinalis.

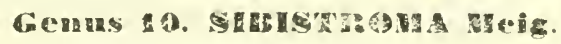

Spec: Typ: Doucopts Obsch malus Meig.

NX. Vena transversa ordinaria proxina margini postico alerum, seu longa circiter ut distantia ab apice long:Indinalis quartic.

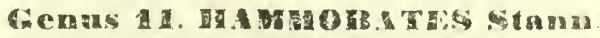

Sper: Typ: Havronates Notates Slanuills. 
MM. Arista articulo penultimo non aut parum elongato.

O. Proboscis valde elongata.

\section{Genus 2. OETOCHIE catr.}

\section{Spec: Typ: Ort. Nigrocoerula Latr.}

00 Proboscis non distincte elongata.

P. Tarsi postici articulo basali spinis longis instructo prexter apicales.

Q. Vena longitudinalis quarta fracta et appendicula prædita in parte exteriori.

\section{Genus 13. GaGHeveUa A MH.}

Spec: Typ: Dozicopus Griserpennis Stann.

QQ. Vena longitudinalis quarta extrinsecus nec fracta nec appendiculata, aliquando tantum paulo flexa vel sinuosa.

\section{Genus 1. ACMANTIPOBES Mihi.}

Spec: Typ: Dolicopus Regalis Meig.

PI. Tarsi postici articulo basali spinulis nullis prædito exceptis apicalibus.

Genus 15. Dordeopos latr.

Spec: Typ: Dolicopls Nigrllamelatus Mgn. 
Stirps XXXVIII. RAPHIINA Rndn. ( $\left.{ }^{*}\right)$

raphidi Bigot. - Cætera Synon: ut in familia byLICOPIDORUM.

A. Antcma arliculo tertio brevi vel subcirculari vel subsonico vel breviter sublanceolato in utroque sexn.

B. Tibia posticæ non spinulosæ.

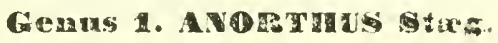

Spec: Typ: Medeterus Jaculus Mg"

BB. Tibice posticæ magis vel minus spinulosæ.

C. Oculi non manifeste pilosi nec tomentosi.

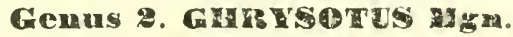

Spec: Typ: C. Elegans Hgn.

CC. Oculi pilosi vel manifeste tomentosi.

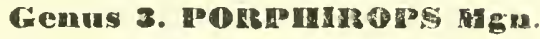

Spec: Typ: P. Elegaktulua Mgn.

AA. Antenne articulo tertio satis elongato saltem in mare, s:epius in femina lanceolato.

(i) Questa stirpe forse conveniva di lasciarla unita allo DourcoPixe, una ho credulo di doverla separare, perchè anehe in altre famiglie, la posizione della sctola all' estremità delle antenne, accompagnata da forma distiata degli oryani stessi, furono motivo per dividerle in diverse st irpi. 
$1 / 6$

1). Anerma articulo tertio basi valde dildtate, extrímecus cylindrico et paulo attenuato ad apicem.

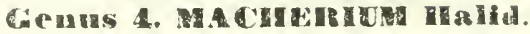

Sper: Typ: M. Hantruuda Halid.

ID. Antennee articulo ulimo ensiformi in mare, et lanceoluto sappius in fæmina.

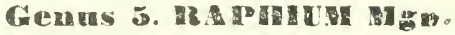

Spec: Typ: Rapilum Caliginosu Meig.

Stras XXXIX. TACHIDROMYNA Rndn.

tachroronydes p. Wiedm. Zett. - tachidrourd. p. Weslw. tacubromy p. Meig.

A. Areolu analis completa.

B. Anterme articulo tertio ensiformi. - Stilus apicalis, brevis, saltem bievior articulo tertio antonnarnm.

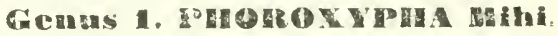

Spec: Typ: Tacuidronya Longicornas Meig. 311. Antenna articulo tertio non ensiformi - Stilus vel sefer saltem longiores articulo tertio antennarum.

Genus 2. TCHibony neig.

Spec: Typ: Tachidonya Flavipes Meig. 
1. Areola analis nulld aut incompleta.

C. Antenna arista longa præditæ.

D. Femora antica manifeste incrassata.

E. Alce longiludine ordinaria.

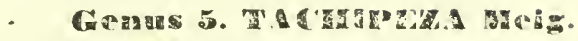

Spec: Typ: 'Taculpeza Nervosa Meig.

SE. Ale Valde abbreviatx.

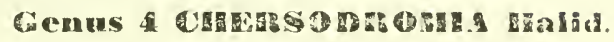

Spec: Typ: 'TAcmipeza Br.fulpennis Zett.

10). Femorce antica ut sequentia non manifeste incrassata. G

Spec: Typ: Drapetis Exilus Meig.

(c. Antemnce Stilo brevi apicali.

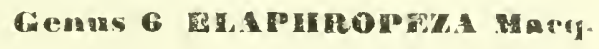

Spec: Typ: Emerodromy Epupprats Heig.

STIRPs XYXX PHYLLODROMYNA Rndu.

Symon: Stips Tacudronyanem.

A. Temulu oúliqua nulla conjungens costalem ei terl iam lolie silutinalem. 
B. Venula "nullæ transversarix in medio alarum, sed tres ante medium seu in parte basali.

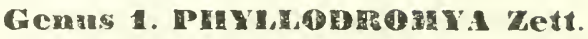

Spec: Tyjp: Tachidromya Melanocepuala Fabr.

LB. Vera aliqua Iransversaria, saltem una in medio aiarum sita, præter basa!es.

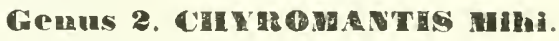

Spec: Typ: Tachidronya Vocatoria Fall.

AA. Venula obiiqua adest conjungens costalem et venam tertiam longitudinalem.

C. Venula transversaria unica in medio circiter alarum adest.

\section{Genus 3. HEMERODROMY Hodm.}

Spec: Typ: Tachidronya Oratoria Fall.

CC. Venula transversarice duæ ve! tres in medin circiler alarum adsumt.

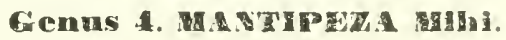

Spec: Typ: Нелеrogenya Morostygua Hoffim. 
Strkps XXXXI. EMPIDINA Rindn.

expidu Fall. Meig. Zett. - enrpides Latr. Maci. Ełplo:s. Westw. Rndn. Bigot. Walk.

4. Vena longitudinalis tertia brevis vel brevissina, aliquando obliqua et fere transversa cun sequente furcam venosam constituens; rarissimo basi interrupta.

B. Fenœ longitudinales secunda et tertia versus apicem. conjunctie venula transversaria.

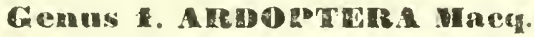

Spec: Typ: IIeverodroury Irrorata Meig.

BB. Venula transversaria nulla inter secundam et tertiam longitudinales.

C. Arista satis longa, alt saltem longior articulo tertio. antennarum.

D. Furca venosa sub-apicalis ad basim venula transversaria intersecata.

\section{Genus 2. CundCenA Meig.}

Spec: Typ: Cuivocera Nigra Mign.

DD. Furca venosa sub-apicalis. non intersecata ad basim venula transversaria.

s. Furca venose ramulus superior basi interruptus Antenne articulis duobus extremis sphærulam contstituentibus.

Genus 3. GuOWh Hein.

Spec: Typ: Glowa Euscipenvis Meig: 
2. Furce venosa ramulus superior basi non interruptas - Antenne articulis duobus ultimis forma ordinaria.

17. Antema articulo ultimo brevi, et cum arista cubitum non efficiente. - Tibice subnudæ.

6. Alarum areola intermedia (discoidalis) longior interioribus (basilaribus) in utroque sexu, - Abdomen maris apice non vesciculoso.

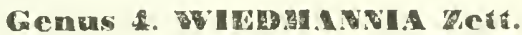

Spec: Typ: Wiemanim Boreals Zelt.

6G. Areola intermetia alarma isevior interioribus $-1 b-$ domen maris apjec venciculoso.

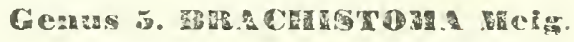

Spec: Typ: Bacca Tescicllosa Fabr.

FF. Antemox articulo tertio longo, attenuato, et cum arista cubilum efficiente - Tibia setis Jongis prodita.

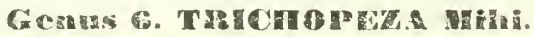

Spec: Typ: Bracinstona Longloconns Mgu.

CC. Arista brevis vel brevissima saltem breviol articula tertio antennarum.

H. Proboscis bresviuscula, ad summum longiludine capitis, et si raro parum longior, tunc crassiuscula et sub membranosa.

1. Vena longitudinales tertia et (quarta transversaria subapicali conjunclæ.

\section{Gemus y. DR IODRo.hy Mitei.}

Spec: Typ: (nova) Dryodrouya Testacea Milli. 
11. Vena transtersaria nulla inter tertian et cqualtain tongiludiniles, ad apicem.

Gentus 8. HTLAR:A Meim.

Spec: Typ: Asllis maxicatus Geofr.

111. Proboscis exilis et, cornea, sæpius longior capite.

K. Femora postica distincte incrassata.

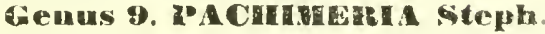

Spec: Typ: Eupts Fenorata Fabr.

hK. Femora postica non distincte incrassata.

Genas 10. Whis' Meig.

Spec: Typ: Ehpls Tesellata Fall.

A A. Fena longitudinalis tertia forma et longitudine ordinaria et furcam venosam non efficiens ad apicem alurum.

1. Antennce stilo brevi apicali longitudine ad summum anticuli tertii antennarum.

3. Proboscis saltem longitudine capilis.

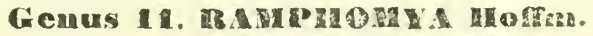

Spec: Typ: Eupts Sulcata Fabr.

MIM. Proboscis brevissima aut brevis, ant saltemt distincte brevior eapite.

צ. Areola intermedia alarum (discoidalis) adest.

(2). Vena longitudinalis prima costali conjuncta contra vel ultra apicem areole eliscoidalis.

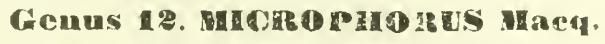

Spec. Typ: Microphonus Veutines Haci. 
$13: \underline{2}$

40. Pena longitudinalis prima costali conjunta satis ante apicem areole discoidalis.

\section{Genus 13. TRICHIV. Meig.}

Spec: Typ: Tricuiva Cravipes Meig.

NN. Areola intermedia alarum non adest.

Gemus 14. CInTOMA Mgr.

Spec: Typ: Eupis Pluicaria fall.

STIRPS XXXXIl. IIYBOTIDINA Rndn.

Hувотпе Latr. Macq. Westw. Zett. Bigot. - нивоmixz Meig. Zett. - Cot. Syn. Stirpis Empidixarur.

1. Areola ultima postica alarum (analis) brevior præcedente. 3. Areola intermedia (discoidalis) venas tres in spatio margrinali emitteus.

Genus 1, GWALEA Mgm.

Spe: Typ: CEulea bracata Mihi.

BB. Areola intermedia venas duas tantun in spatio marginali emittens.

Genus 2. PTEReSPILCS 是ih:

Spec: Typ: Hyos Muscarius Fabl. 
A1. Areola analis longior priecedente.

Genus 3. HY

Spec: Typ: Hygos Infuscates Zett.

Stikps XXXXII. LEPTIDINA Rndn.

leptides Meig. Macq. Zett. - ragionides Latl. leptide Westw. Walk. - anthascide p. Fall. gydasI p. Latr. - zeptine Rndn. - Leptidi Bigol.

A. Arista manifeste apicalis.

B. Areola analis apice clausa.

\section{Genis a. CERTEPLA Reg.}

Spcc: Typ: Atuerix Dandera Fabr.

BB. Areola analis in margine alarum aperta.

c. Antennarum articulus tertius longior præcedente. - Vena longitudinalis quarta brevis et secunenti conjuncta ad basim, longe ab arcola discoidali.

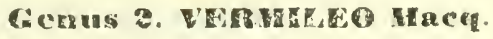

$$
\text { Spec: Typ: }\left\{\begin{array}{l}
\text { Lepres Vermileo fah. } \\
\text { Veruleo Degeenu Maeq. }
\end{array}\right.
$$

CC. Antennarum articulus tertius bresis circiter ut pracedens. - Vena longitulinalis quarta longinscula et 
15 '

oriens a sequente contra apicem areola discoidalis.

\section{cácus a. LEPTIS Fabr.}

Spec: Typ: Musca Tringaria Lin.

1A. Arista apparenter dorsualis, quia articulus tertius antennarum inferne dilatatus.

1). Vence longitudinales decima et undecima conjunclæ ante marginen alarum.

\section{Geums 4. IBBSA Mini.}

Spec: Typ: Bibio Marginata Fabr.

טD. Vena longitudinales decima et undecima sejnnctim margini alarum productæ.

Genus 5. ATEERIX Meis.

Spec: Typ: Atuerix Pilosa Megrl.

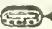

StrRps XXXXIY. SPANIINA Pindn.

heptides p. Macq.

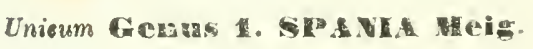

Spec: Typ: Spania Nigra Meig. 
STlars XXXXV. ThereVixd Rn?n.

xýtome Meig. Macq. Lòèw. - therevid.e Westu.

- anturacini p. Fall. - therevine Rndu. - bonBYLIDE p. Bigot.

A. Vence longitudinales octava et nona sejunctim margini alarum productx. - Antemce articulo primo distincte incrassato.

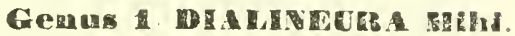

Spec: Typ: Miusca Anulus Lin.

A. Vence longitudinales octava et nona conjunctie ante marginem alarum. - Antennce articulo primo non manifeste incrassato.

Genks 2. Th

Spec: Typ: Musca Plebeja Lin.

STIR's XXXXVI. PACHISTOMINA Rindn.

SICARII p. Latr. Maeq.

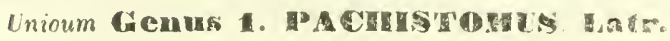

Spec: Typ: Ragio Syrpholdes Panz. 
Stmps XXXXVI. DaSIPOGONINA Rndn.

dastpogonuz Bigot. - Coeter. Synon: Famil. asmivoruar.

1. Areole analis ante marginem clausa, petiolo apicali magis vel minus distincto.

13. Veno longitudinales octava et nona sejunctim margini postico alarum productie.

C. Tarsi ut tibice postica non incrassati.

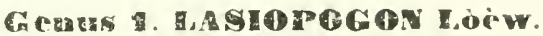

Spec: Typ: Dasipogor Hirtelus Meig.

CC. Tarsi ut thim posticx distincte incrassati.

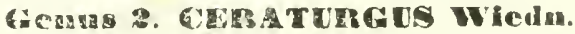

Speu: Typ: Asurus Verustus Rossi.

BB. Vene longitudinales octava ct nena ante marginem. alarum coninuclac.

D. Vena longiudinales quinta el sexia sejuuctim margini alarum productæ.

\section{Ge.}

Spec: Typ: Dasirogon Fimbriatus Meig.

DD. Vence longitudinales quinta el sexta ante marginem alarun conjunctæ.

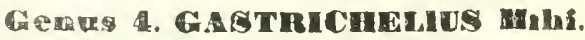

Spec: Typ: (nova) Gastrickrelus Harigtivorus Mihi. 
A. Areola analis in margine alarum aperta, et si in ipsu margine clausa petiolo apicali non distincto.

L. Proboscis horizontalis vel sub-horizontalis, seu antice porrecta non pendens.

F. Fucies tota barbata inter epistomium et antennas.

6. Vena transtersa antica circiter æequidistans ab origin: venarum longitudinalium quartæe et quintæ. - Stilus antennarum exilis et longiusculus.

\section{Genus 5. PyCropocior Lòèw.}

Spec: Typ: Pycnopgen Mixtcs Lòew.

GG. Vena transversaria antica magis proxima basi vente quintie longitudinalis, quam basi quartie. - Still6s brevis et crassiusculus.

\section{Genus 6. Cytropocor Lùèw.}

Spec: Typ: Asilus Ruficonnis Fubr.

FF. Facies in parte infera tantum barbata.

H. Pulvilli inter uncos tarsorum distincti.

I. Tibice antica unco apicali terminante.

K. Vence longitudinales octava et nona sejunctim margini alarum productæe.

\section{Genus \%. Maslyogori Fabr.}

pec: Typ: Dasipcgon Tector Fabr.

KK. Tenoe longitudinales octava el nona conjuncie anto marginem alarum.

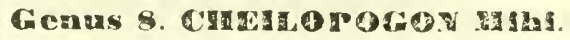

Spec: Typ: Dasipogen Puxctatus Fabr. 
138

II. Tibia antice unco apjicali destitutæ.

i. Vena longitudinalis octava ad apicem statim flexa et cum seqquente in margine alarum conjuncla.

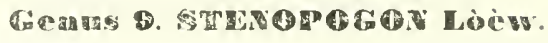

Spec: Typ: Dioctria Sabieda Fabr.

LL. Yenu longitudinalis octava margini alarum regulariter producta sejunctim a nona.

\section{Gemus 10. PHoCtria gabr.}

Spec: Typ: Dioctria Linearis Fabr.

HH. Pulvilli inter uncos tarsorum non manifesti; loco pulvillorum setula adest.

Geans 14. GONHES latr.

Spec: Typ: Dasipogon Tipuzoides Fabr.

E⿰氵. Proboscis subperpendicularis scilicet non erecta, sed pendens.

II. Tarsi arliculo basali satis parvo, circiter breve ut sequentes sejunctim. - Antennce articulo ultimo bre-viusculo et sub-lanceiforme.

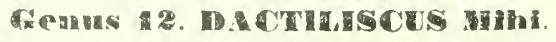

\$pec: Typ: Asulus Strutus Fabr.

My. Tarsi articulo mimo satis longiore sequentibus sejnn- 
clim. - Antenne articulo ultimo satis elongato subensiforme.

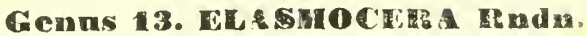

Spec: Typ: Asilus Graucius Rossi.

Straps XXXXVHII. ASILINA Rndn.

asilide Bigot. - Cæater: Synon. Tamil: Asılnon

A. Vena longitudinalis quarta, basi quintæ conjuncta.

\section{Genus 1. Antus lira.}

Spec: Typ: Asilos Germinicus Lin.

AA. Vena longitudinalis quarta, basi terliæ conjuncta. Genus 2. TRUPANA Alach.

Spec: Typ: Trupanea Cyprica Bellardi (in litteris). 
160

STIR XXXXIX. LAPHRiNA. Rndn.

lapurttide bigut. - Coeter: Synon: Famil: asulboriar.

A. Fena longiludinales tertia et quarta venula transrersaria" conjunctie.

\section{Genus 1. POG ONOSOAI Mibi.}

Spec: Typ: Lapuria Maroccana Fabr.

AA. Vence longitudinales tertia et quarta non conjunetæ venula transversaria.

3. Tence longitudinales quinta et sexta sejunctim margini alarum productæ.

\section{Gerus 2. Maphata Fabr.}

Spec: Typ: Laplirha Marginata Fabr.

Bb. Vence longitudinales quinta et sexta conjuncta andé urarginem alarum.

Genus 3. AXBrevosoma Mih.

Spec: Typ: Laphria Atra Fabr. 
Splaps L. MY DASINA Rndn.

Synun: Famil: Mydasidahum.

\section{Unieum Genus 1. MIDAS Fabr.}

Spec: Typ: Mrdas Lusitanicus Iloffg.

Stikps LI. FALLENINA Rndn.

nemestrinide Macq. - anturacit p. Katr. - bokBXLLARII. p. Meig. Wiedm.

A. Alce ad apicem reticulatæ.

\section{Genus 1. NeMESTIRA Latr.}

Spec: Typ: Nemestrina Perezil Dufour.

A.1. Alce ad apicem non reticulatx.

B. Fence longitudinales quinta et sexta sursunı incurvalse et apice in quuarta eonjunctæ.

\section{Genus 2. Fardevia Mga.}

Spec: Typ: Crterea Fasciata Fabr.

BB. Vence longitudinales quinta el sexta pracedentibus sub 
parallelie et margini sejunctin productx.

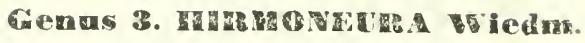

Spec: Typ: Heruoneura Obscura Mgn.

STIRPS LII. BOMBYLINA Rndn.

Synon. Famil: Boubylidarug exceptis Nerrestrinimis Macq.

A. Vence longitudinales aJarum saltem decem, prima considerata etiamsi aliquando parum distincta.

B. Antennarum articulus primus erassitie ordinaria et dislincte brevior tertio.

C. Vena quarta longitudinalis quintæe et tertiæ ad basim connexa.

D. Vence longitudinales quinta et sexta conjunctæ ante marginem alarum.

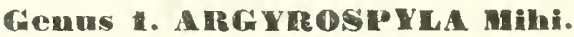

Spec. Typ: Anturax Jaccus Fabr.

DD. Vence longitudinales quinta et sexta sejunctim margini alarum productæ.

E. Proboscis non elongata vel saltem distincte brevior capite.

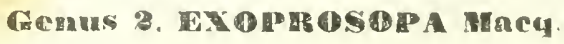

Spec: Typ: Anturax Capucina Fabr. 
EF. Proboscis elongata, saltem manifeste longior capite.

Genas 3. Whete tat?

Spec: Typ: Crtherea Obscura Fabr.

C.C. Vena longiludinalis quarta ad basim quintx tantum conjuncta.

F. Antennarum articulus primus brevis, ad summum longitudine dupla secundi.

G. Vence longitudinales nona et decima sejunctim margini alarum productæ.

H. Proboscis eliongata, saltem longitudine capitis.

Genes 4. Geosist A Mihi.

Spec: Typ: Mulio Infuscatus Mgn.

1111. Proboscis brevissima aut saltem brevior capile.

1. Fena tertia longitudinalis a quinta oriens satis interius, ante apicem areola basilaris anterioris.

\section{Genus 5. LOMA TH Mgn.}

Spec: Typ: Anturax Sabea Fabr.

II. Vena tertia longitudinalis oriens a quinta circiter contro apicem areole basilaris anterioris.

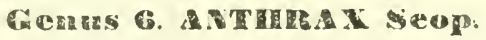

Spec: Typ: Axтиnax Monio Fabr.

G(i. Tence Iongitudinales, nona et decima conjunctie ante marginem alarum.

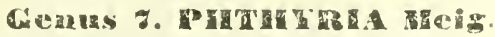

Spec: Typ: Volucelua Minuta Fabr. 
WF. Antemarum articulus primus elongatus, saltem trilongior sape quarlrilongior et ulra secundo.

K. Tence longitndinales quinta el sexta sejunctim margin: alarum produclie.

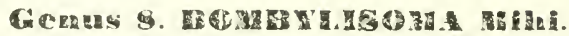

Spec: Typ: Bombylus Sulpuureus Fabr.

hK. Fence longitudiuales quinta et sexta conjunctie ante marginem alarum.

\section{Gercs 9. Bose}

Spec: Typ: B: Ater Lin.

bB. Antema articulo primo satis incrassato et Jongiore lertio L. Areola duæ basilares subæque longæ.

\section{Genus 10, PLOA Eatr.}

Spec: Typ: P. Grised, Fabr.

1.4. Areola basilaris antica satis elongata ultra apicem posterioris.

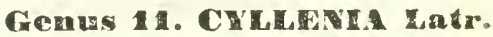

Spec: Typ: C. Macilats Lall.

A A. Fence longitudinales alarum novem tantum, etiamsi prima considerata, spuria vel non.

1. Antemnarum articulus secundus Jongior tertio breviusculo - Antennce proboscide longiores.

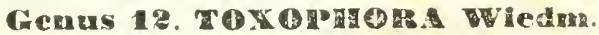

Spec: Typ: Toxopeora Maculata Wiedm. 
MM. Antennarum articulus secundus satis brevior tertio.

N. Veno longitudinales sexta et septima venula transversaria conjuncta.

O. Antennarum articulus basalis satis brevior secundo. Stylus apicalis indistinctus.

Genas as. Geron IIorts.

Spec: Typ: Geron Grbbosus Hoffg.

00. Antennarum asticuli duo primi brevitate parum diversa - Stilus apicalis, sed distinclus.

\section{Genus 14. USIA Eatr.}

Spec: Typ: Volucella Florea Fabr.

NN. Fena longitudinales sexta et septima venula transversaria non conjunctæ.

\section{Genus 15. CIRTOSIA terris.}

Spec: Typ: Cyrtosia Marginata Perris.

Stires LIII. CIRTINA Rindn.

Srnon: Famil: Cyrtidorum.

Unicum Genus 1. Crites Latr.

Spec: Typ: Acrocera Gibba Fabr. 
166

Grmes LIY. HENOPIDINA Rndn.

Synon: Famil: Cyrtoorcs.

4. Antennce in fronte longe ab ore silie.

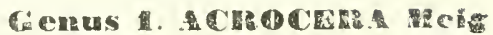

Spec: Typ: Henops Globulus Fall.

A. Antenna prope orem insertc.

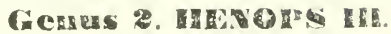

Spec: Typ: Hexops Gibbosts Fabr.

Stips LV. ASTOMELLIVA Rndn.

Synon: Famil: Cyrtidorum.

Unicum Genus 1. ASTOMEIC Dufour:

Spec: Typ: Astomella VAxelu Klig. 
Strpes LVI. SCENOPININA Rndr.

Syson: Famil: Scenoprndorery.

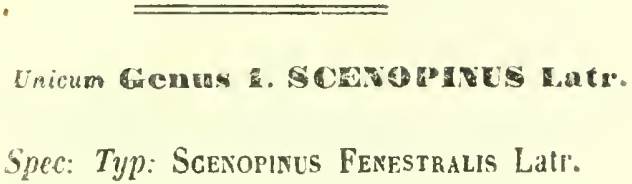

StITPs LVII. CHRYSOMYNA Rndn.

Synon: Famil: Strationydarun.

A. Oculi nud!.

B. Epistomium satis productnm, subrostriforme; - Proloscis it epistomium elongata.

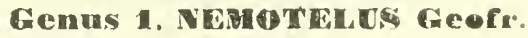

Spec: Typ: Musca Uliginosa Lin.

BB. Epistomium ut Proboscis breve.

C. Areola duce interiores (Basilares) in unica subconfuse quia venula sub indistincta separatæ. - Vence spurixe productæ in spatio marginali tres tantum.

Geuns 2. PACHGASTh Meig.

Spec: Typ: Vappo Ater Latr. 
168

C.. Arcola due basilares rena dislinctissima divise Venee spurice producta in spatio marginali quatuor.

7. Arista in utroque sexu apicalis. - Oculi in mare contigui superne.

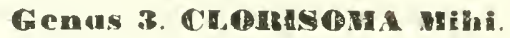

Spec: Typ: Sargus Pallipes Meig.

D. Arista dorsualis. - Oculi in fronte distantes clianl i. mare.

Genus 4. SATreds rabe.

Spec: Typ: Musca Cupraria Lin.

AA. Oculi hirti.

E. Arista sub-apicalis filiformis vel setiformis, et basi pi losula.

\section{Gemus 5. CHIt YSOMY Mncy.}

Spec: Typ: Nemotelus Xantopterus Degeer.

FE. Stilus apicalis crassiusculus, basi nudus.

Genus 6. CYCLOGASTER Mac⿱

Spec: Typ: Nerotelus Villosus Fabr. 
StiRPs LVIII. ODONTHOMIN. Rndn.

Synon: Famil: Strationydarur.

A. Scutellum sexdcntatum.

Genas 1. DX D D THA Bellardi.

Spec: Typ: (nova Exodontua Pedbanontana Bellardi (in litteris).

A: Scutellum (in indigenis) bidentatum.

B. Arista sub-apicalis exilis et elongata.

Gentas 2. Oxychen neig.

Spec: Typ: Oxicera Leonixa Panz.

BB. Antennce stilo brevi apicali vel subnullo.

C. Oculi hirti.

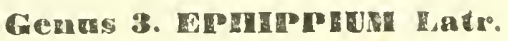

Spec: Typ: $\left\{\begin{array}{l}\text { Stratiomys Euphopicia Fabr. } \\ \text { Ephopium Thoracigum Latr. }\end{array}\right.$

CC. Oculi nudi.

1). Antennce articulo primo salis elongato. - Stilus apicalis vel brevissimus vel subnullus.

Genus athatrongs Geomro

Spec: Typ: Strationirs Srrigata Fabr.

DD. Anterme articulis duobus primis sub-reque longis. - 
Stilus apicalis satis dislinclus.

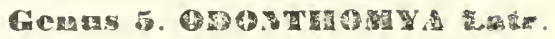

Spec: Typ: Musca Hydromen Fabr.

StikP LIX. TABININA Rndn.

Synon: Famil: Tabaninorum.

A. Articulus tertius antennarum basi non distinctissime dilatatus, et superne nou manifeste excavatus.

B. Antennarum articulus tertius in articulos distinetos diaisus: inde antennæ sex arlictilalæ.

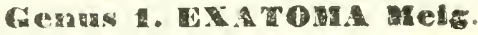

Spec: Typ: Exatoma Bimaculata Meig.

BB. Antennce triarticulatæ, articulo tertio circulatim inciso saltem ad apicem sed in articulos distinctos non diviso.

C. Ocelli non manifesti.

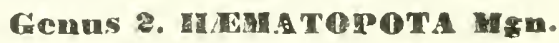

Spe: Typ: Tabanus Pluvials Lin.

CC. Ocelli manifesti.

D. Antennarum articuli duo primi longiludine subæquales.

GCans 3: CIITISOES Melg.

Spec: Tup: Tabanus Relictus Gcofi. 
oD. Antennarum articulus secundus satis brevior primo.

E. Antennarum articulus tertins longitudine circiter æequalis præcedentibus conjunctim; secundus latitudine longior.

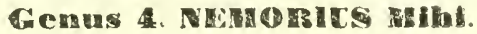

Spec: Typ: Curysops Vitrupennis Meig.

E. Antennarum articulus terlius satis longior præcedentibus conjunctim: Secundus sub-æque latus et longus.

\section{Genus 5. STLVUS Meig.}

Spec: Typ: Tabanus Vitul Fabr.

A. Ar. Articulus tertius antennarum basi dilatatus et superne excavatus.

\section{Genus 6. TA Baves Rilu.}

Spec: Typ: Tabanus Bovinus Lin.

Srinps LX. PANGONINA Rndn.

Synon. Famil: Tabanidoruz.

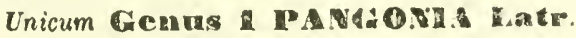

Spec: Typ: Pangonia Magulata Fabr. 
STIRPS LXI. CCENOMYNA Rndn.

coeromydz Westy. - sicaril Lalr. Mact. - sicaRID.s Bigot.

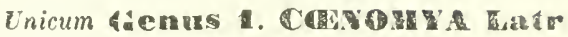

Spec: Typ: Sigus Fraruanecs Fabr.

StiRPS LXII. XYLOPHAGINA Rndn.

xylopiagir p. Fall. Meig. Lalr. Macq. - Xylopagide Bigot.

1. Antennarum articulus primus satis longior secundo.

\section{dents 1 XYOPRAGUS Meig.}

Spec: Typ: Xylophagus Ater Fabr.

A. Antemnarum articulus primus brevis circiter ut secundus. Genas 2. SubuLA Megri.

Spec: Typ: Subula Varia Megr. 
STIRPS LXIII. BERIDINA Rnim.

xylophagil p. Meig. Macq. - beride Westw.

A. Areola intermedia alarum venulas tres integras et unam: rudimentariam in spatio marginali emittens. - Scuttellum quadridentatum.

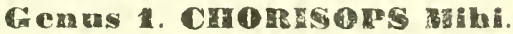

\section{Spec: Typ: Beris Tibialis Mgn.}

AA. Arcola intermedia alarum venulas in spatio marginali emittens duas vel tres, vullaque rudimentaria. Scutellum sexdentatum.

\section{Genus 2. Heiris carr.}

Spec: Typ: Beris Vallata Fabr.

Stirps LXIV. BIBIONINA Rndn.

bibionides p. Macq. Westw. - bibionide p. Macq. Bigot. Walk. - ilrteides Zelt. - Bibionive Rndn. p.

A. Tibio antice simplices, scilicet nec apice unco valids instructæ, nec transversim spinuliferæ.

\section{Gents 1. Pentetata Meig.}

Spec: Typ: Pratetria Holosericea Meig. 
$17 k$

AA. Tilia antica vel unco valido ad apicem instructa, rell transversim spinuliferæ

A. Tibia antica seriebus spinulornm transvèrsariis non iastruclæ, sed tantum unco valirio apicali.

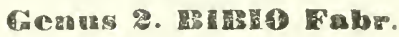

Spec: Typ: Bibro Margi Mign.

BB. Tibice antice seriebus spinulorum transversariis instructix.

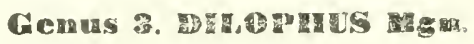

Spec: Typ: Dhopuus Ferroratus Micig.

SrIRPS LXV. SCATOPSINA Rndn.

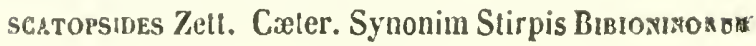

A. Femora antica subtus spinulosa, seu denticulata.

Genus 1. Astisteg Horg.

Spec: Typ: Aspistes Berolinensis Hoffg.

A. Femora antica inferne non denliculata.

Genus a. SCATOPSE Gcorr.

Spec: Typ: Tipula Notsta Lin. 
3Tirs LXVI. SIMULINA Rndn.

8imuLides Zell. - stmuLine Rndn. - shaudde Walk

- Tip. Latipennes Meig.

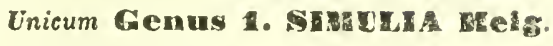

Spec: Typ: Simulu Ornata Meig.

Entsps LXVII. CHIRONOMINA Rndn.

chironomides Macq. Westw. - carronom Zett. cMronomine Rndn. - CHHONoMide Walk. - T. cenCiforaes Meig. Macq.

A. Femora antica nisi omnia subtus spinulosa.

B. Antennoc in mare non plumatæ sed ut in fœmina breviter verticillato-pilosæ.

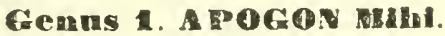

Spec: Typ: Ceratopogon Hontulanus Meig.

BB. Antennce in mare plumatæ, in fomina verticilla: pilosæ.

\section{Cemus 2. SERmony Hath.}

Spec: Typ: Ceratopogon Femoratus Meig. 
A. Femora omnia non spinulosa inferne.

F. Tarsi postici articuio prino longitudine maxima, tibia. longiore; antici et internedii articulis duobus penultimis dilatatis.

\section{Gentas 3. HACAORTI Mgn.}

\section{Spec: Typ: M. Alpitansis Mgn.}

EE. Tarsi postici articulo primo tibia satis breviore. Antici et intermedi non dilatati.

C. Antennce articulis tredecim vel quatuordecim in utroque sexu.

1). Antennce maris in parte basali tantum plumatic. - Veno longiludinales alarum duæ posteriores furcam venosam constitnentes et ad apicem divergentes.

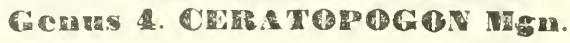

Spec: Typ: Ciclex Pulcaris Lin.

DD. Antenrace maris fere usque ad apicem plumatæ. - Vance longitudinales dnæ posteriores furcam venosam non constituentes et ad apicem non divergentes, sed convergentes.

\section{Genus 5. TANYRES Meig.}

Spec: Typ: Tipula Monilis Lin.

C.C. Antennœ maris articulis tredecim, fœminæ sex tantuo instrucix.

Genus 6. Chmoromus Meig.

Spcc: Typ: Tupula Plumosa Lin. 
Strnts LXVIII. CORETHRINA Rnản.

Srmon: Slippis Cumonominarum.

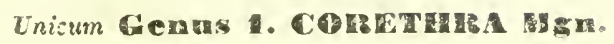

Spec: Typ: Coretura Flayicans Mgn.

Stirps LXIX. CUliCiNa Rndn.

culicides Latr. Macq. Zett. - culcisæ Zétt. Rndn.

- culicide Westw. Bigot.

A. Palpi in utroque sexu proboscide satis breviores.

\section{Genus 1. ARE ES Hoffe.}

Spec: Typ: Aedes Cinereus Hoffm.

A'a. I'alpi longitudine circiter proboscidis saltem in mare. B. Palpi in mare tantum fere longi ut proboscis, in foc-mina valde breviores.

Genus 2. CuLEX Lin.

Spec: Typ: Culex Pipiess Lin. 
BB. Palpi in urroque sexu longi circile! ut proboscis.

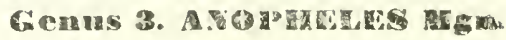

Spec: Typ: Anopheles Maculpenns Hofg.

Sirks LXX. PHLGEBTOMINi Rndn.

PHLABotomide Rndn. Walk. Halid: - pHLEBotowrmas Rndn. - pualenorde p. Macr. Bigot.

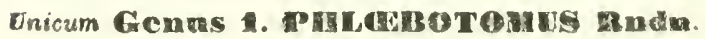

Spec: Typ: Musca Papatasil Scop.

STIkPS LXXI. PSYCODINA Rndn.

Phalenorda p. Meig. Macr. Bigat. - psycodides Zelt.

- rsycodins Rndn. - Pulcebotonide Halid: Walk.

A. Vene longitudinales aliquæ satis longe a basi alarum conjuncta.

\section{Cenas 1. PSICODA Latr.}

Spec: Typ: Tipula Fazenoloes Lip. 
a. Fence longitudinales omnes isque prope basim alaruar. liberæ, seu non conjunclæ.

Genus 2. HY Y

Spec: Typ: (nova?) Lyoveva Lugubris Mihi.

Srisrs LXXII. LINNOBHNA Pndin.

Syron: Famil: Tipulidaruar.

A. Vene longitudinales alarum undecim rel duodecim ad marginem vel fere in margine productæ.

B. Antennce pilosæ, pilis utiamsi brevibus verticillatis.

C. Vence longitudinales undecim tantum margini productæ.

D. Vence longitudinales tertia et quarta sejunclim orientes $a b$ areolis interioribus.

\section{Genus 1. ERIoptera melg.}

Spec: Typ: Erioprera Obscura Meig.

DD. Vence longitudinales tertia et quarta conjunctæ magis rel minus prope areolas basilares.

5. Areola discoidalis nulla.

(") Aa Genus Sacopterix? Halid. 
F. Terla sexia longitudinalis septime non conjuncta extra areolas basilares.

4. Vena ultima postica undulata et longa ut areolæ hasilares.

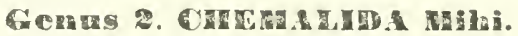

Spec: Typ: Erioptera Toenionota Wiedm.

diG. Vena ultima postica, recta et salis brevior areolis basilaribus.

II. Venula transversaria adest qinæ secundam Iongitudinalem conjungit tertiæ, ubi ista cum quarta connectitur. Furce venosa apicalis ramuli longissimi, petiol o brevissimo.

\section{Gensas 3. RLSOMY Nihi.}

Spec: Typ: (nova) Ilasomya Nubipennis Mihi.

HII. Venula transversaria adest, quæ conjungit secundam longitudinalem quartæ, ante conneclionem istius cum? tertia. - Furca venosce apicalis ramuli non longissimi, et ejusdem petiolus longus saltem ut furcæ ramulus superior.

\section{Genus ilmapmina mihi.}

Spec: Typ: Erioptera Lutea Meig.

FF. Vena longitudinalis sexta, septimæ conjuncta extra areolas basilares.

I. Antenno articulis petiolatis, petiolis longitudine circiter articulorum.

\section{Genus 5. DrMTSE Mihi.}

Spec: Typ: Erioptera Nodulosa Macq: 
11. Antenna articulis sessilibus ant parum distantibns.

K. Venula transversaria adest conjungens secundam et lertians longitudinales ante basim furce venosæapicalis, cuju. ramulus superior brevissimus, et petiolus satis fongus. - Areola basilaris postica satis brevior antica.

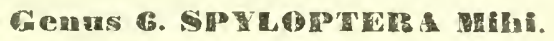

Spec: Typ: (nova) Spyloptera Meridionalis Mihi.

KK. Venula transversaria adest conjungens secundam longitudinalem tertiæ in basi furcæ venosæ apicalis, cujus ramulus superior satis longus, et petiolus brevissimus.

- Arcoloe basilares sub-æque elongatæ.

Gemus \%. Henve a Whin.

Spec: Typ: Eriortera Flayescens Lin.

EE. Arcola discoidalis adest.

L. Vena longitudinalis ultima postica valde sinnosa.

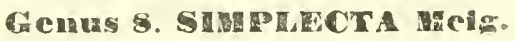

Spec: Typ: Limooria Stictica Meig.

LL. Vena longiludenalis postica recta vel subrecta.

I. Capitis productio longitudine circiter antennarum.

\section{Genus D. ERAMPIIDA Meig.}

Spec: Typ: Lmmobia Longrostris Meig.

MM. Capitis productio valde brevior antennis.

N. Antenno articulis cylindricis.

Genus 10. CVLIN TROTHA Nace

spec: Typ: Lirnobia Distixatissum Mleig. 
182

28. Antenno articnlis subrybulosis vel sub-ovatis.

0. Vena longiludinalis terlia brevissima obliqua, et nea conjuncta secunile venula transversaria.

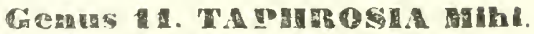

\section{Spec: Typ: Limiveda Tenella Hofg.}

No. Vena longitudinatis tertia longa vel longissima, sequenti subparallela, el secundæ conjuncta venula transversaria.

P. Vena longitudinalis prima ad apicem secundæ conjuncta venula transversaria, et pari modo secunda tertiz conjuncla.

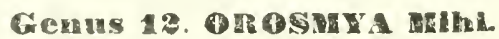

Spec: Typ: (nova) Orosuya Apenmena Mihi.

PP. Vena longiludinalis prima ad apicem non conjuncta secundæ venula transversaria; et secunda tertiæ conjuncta venula transversaria, prope basim furcæ venosæ sæquentis.

\section{Genus 13. IHSEA Mibi.}

Spec: Typ: Erioptera Maculata Meig.

CC. Vence longitudinales dnodecim margini productæ.

Q. Areola discoidalis adest.

R. Areola basilaris pustica, a venula transversaria in duas divisa. - Vena ultima posticit ad apicem subfurcata.

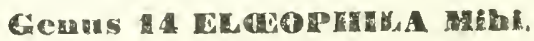

Spec: Typ: Livnobia Maruorata Hofg. 
3R. Arcola basilaris postica in duas non divisa. - Vera ullima a! apicen non subfurcata.

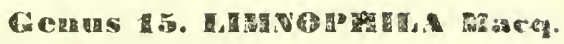

Spec: Typ: Liminobia Puxctata Meig.

QQ. Areola discoidalis non adest.

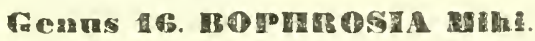

Spec: Typ: Lunobu Imuaculata Meig.

BB. Antennce subundæe vel brsvissime tomentosæ, si raro pilosilæe, lunc pilis non verticillatis.

S. Areola discoilalis adest.

T. Vena ultima postica brevissima et prope basim alarum incurvata - Areola basilaris postica in duas non divisa.

\section{Gemus 16. TRICHOCHRA Metg.}

Spec: Typ: Trichocera Hyemalis Degeer.

Tr. Vena ultima postica longitndine et forma ordinaria Areola basilaris postica in duas divisa.

U. Venoe longitudinales duodecim margini alarum productæ。

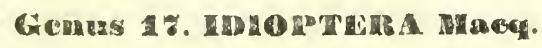

Spec: Typ: Linnobia Fasciata Meig.

UU. Venœlongitudinales undecim margini productæ.

Genus 15. Uta Halks.

Spec: Typ: Ula Mollissima Ilalid. 
S.S. Areola discoidulis non arest.

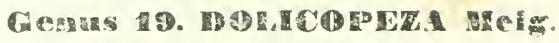

\section{Spec: Typ: Dolicopeza Sylvicola Meig.}

A. Veno longitudinales alarum decem tantum margin: productæ.

V. Areola discoidalis non adesi.

X. Antenna ad summum articulis septem distinctis; lertio quarto et quinto longissimis.

7. Venula radicalis contra basim areolarum basilariurı sita. - Antennce maris longitudine circiter capitis el thoracis; fominæ capite transverso parum aut saltem non duplo longiores.

\section{Gemus 20. ANISOMERA Matis.}

Spec: Typ: Amisomera Nigra Meig.

\%\%. Venula radicalis ante originem areolarum basilarium sita. - Antenna máris longitudine circiter corporis; fominæ capite transverso saltem duplo longiores.

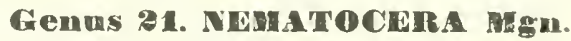

Spec: Typ: Nematocera Bicolor Meig.

XX. Antennce articulis plurimis instructa; tertio, quarto, et quinto etiamsi paulo elongatis numquam longissimis. $\mathbf{Y}$. Antenno seteceæ, nun verticillato-pilosæ.

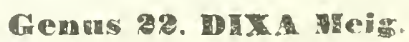

Spec: Typ: Dixa Maculata Meig. 
YY. Antenne filifuimes, pilis breribus sed verticilatis instrucize.

\section{Gerres 23. PELOSI Mihi.}

Spec: Typ: (novia) Pelosia Albifnoxs Mihi.

YV. Arcola discoidalis adest.

W. Productio Capitis longior palpis.

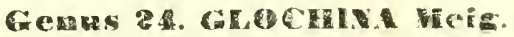

Spec: Typ: Glochima Serrcata Meig.

WW. Productio capilis brevior palpis.

2. Antennce in mare non ramosix; in biroque sexul articulis non divisis a petiolis longiusculis.

a. Vence longiudinales prima et secunda in unica subconfusie, præsertim ad apicem: tertia !ongitulinalis non conjuncta costali nec secunda venula tramsersaria.

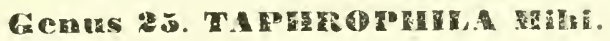

\section{Spec: Typ: Lnowona lnesta Meig.}

aa. Tence longitudinales prima et secunda distinctio etiam ad apicem. - Tertia longitudinalis siepiis conjuncta setundæ, rarius cosiali, remula transversaria.

to. Tena longitudinalis prima satis prodncta nltra originem quartæ.

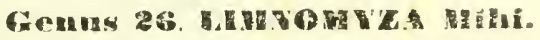

\section{Spec: Typ: Lmanobla Trupunctata Mrig.}

bb. Vena longitudinalis prima costalem attingens circiter contra originem quartie.

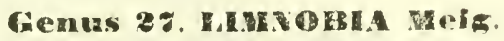

spec: Typ: Lminobia Chorea Meig. 
186

2R. Antenno in utroque sexu arliculis distincte petiolatis; in mare ramosé.

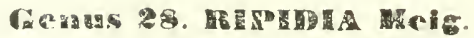

Spec: Typ: Rupuda Macclata Meig.

\section{Straps LXxill, TIPULIN: Rodn.}

Frรon: Famil: Tiptudarur.

1. Anterna in maribus ramosa, in foeminis distincte dentato-serratæe, el si raro non dentato-serratip, the capite transverso vix longiores.

B. Ale areola discuidali proditie.

C. Antenne maris seriéns duabus tantun ramulorum prieditæ, fominæ moniliformes capite transrerso vix longiores.

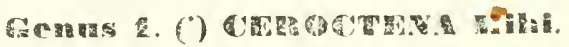

Spec: Typ: Tiplla Buacelata Lim.

C. Anteme maris seriehts iribus vel quatuor ramulorum instructa; fæminæ magis vel minus distincte serratodentati.

(i) An Graus Diengriliu? Brullè. 
D. Antenne maris seriebus tribus ramulorm. - Audomen fominæ in formam oridsti valde elongatum.

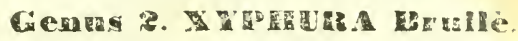

Spe: Typ: Tiplea A trata Lin.

DD. Antenna maris seriebus quatuor ramulorum. - Ablomen founine longitudine ordinaria.

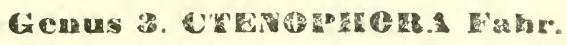

Spec: Typ: Ctenopuora Fldverdis Fubl.

SB. Alce areola discoidali destitutio.

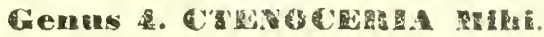

\section{Spec: Typ: P'ticoptera Pectinata Hacr:}

A. Antennce in maribus non ramose, in foeminis non dentato-serratæe ct capite transverso distincte longiores.

E. Antenne articulis a 16 ad 19 instrictix.

F. Al $\propto$ areola discoidali destitutie.

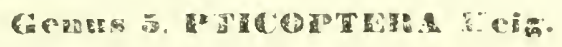

Spec: Typ: Pticoptera Albuhasa Falu.

fi. Ale areola discoidali prestitit.

(i. Antentre articulis 16 irsiructæ.

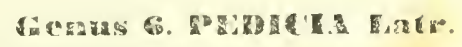

Spec: Typ: Trecla Rrosa lim.

GG. Intenme articulis 19 instructie.

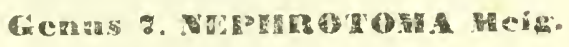

Spec: Typ: Tirea Doisals Fabr. 
188

EE. Antenne articulis duodecim vel tredecim instructie.

II. Alæ in utroque sexu completæ. - Halteres maris satis breviores thorace - Antennace breviores capite ef thorace coujunctim.

I. Aloe areola discoidali destitute.

\section{Genus 8. AtorilnoIDA Mihi.}

Spec: Typ: Alophroloa Canerea Mihi (nova).

II. Alæe areola discoidali præditæ.

K. Vence longitudinales sexta et septima sejunctim ab areola discoidali orientes, eî si raro conjunctæ paulo extra areolan ipsam, tunc vena prima longitudinalis satis producta ultıa originem quartæ.

\section{Genus 9. PACHYRHia Hach.}

Spec: Typ: Tipula Maculosa Hoffg.

KK. Vence longitudinales sexta et septima conjunctie extra areolam discoidalem. - Vena longiludinalis prima, secunda conjuncta coutra originem quartæ.

Gearas 10. TIPULa Lim.

Spec: Typ: Tiplla Oleragea Lin.

HH. Alce in fuemina abortivet. - Halleres maris longitudine thoracis. - Antemne in eodem sexu longiores capite et thorace conjunctim.

Genus 1. P'Tremachises Rndu. Spec: Typ: Pterelachisus Berteji Rndr. 
Stirps LXXIV. CHIONEINA Rmin.

T. terricoles p. Macq. - el cæt: synon: Famil: ChoNEIDARUM.

\section{Unicum Gonus 1. CHIONEA Dalm.}

Spec: Typ: Chionea Araneol bes Dalm.

Stikps LXXV. ORPHNEPHILINA Rndn. Synon: Famil: Orpunephilidarur.

Unicum Genus 1. ORpxnerhak Malfd.

Spec: Typ: Oapunepula Devia Halid. 
190

StiRps LXXVI. BERTEINA Rndin.

Absque Synona:

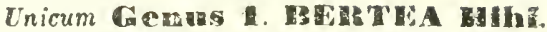

Spec: Typ: Bentea Subaptera Mihi.

STRP LAYVII. ASTHENIA Rndr.

Absi: Srnov:

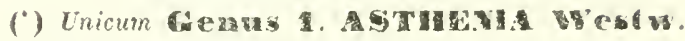

$$
\text { Spec: Typ: }\left\{\begin{array}{l}
\text { Astenia Fasciata Westw. } \\
\text { Begpharicera Limbipennis Macro. }
\end{array}\right.
$$

(") In questa Famiglia si deve faeilmente collocare il genere Macropea del seig. 
Strps LXXVIII. RHYPHive Rndn.

Synon: Famil: Rhypundrum.

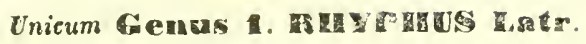

Spec: Typ: Rhypus Fenestrans Meig.

StIRPS. LXXIX. CEROPLATINA Rndn.

Synon: Famil: Mycetophllidarier.

A. Palpi articulo unico crasso.

1). Yena longitudinalis prima satis prodtcta uitra apicem areolse basilaris - Vena longiludinalis tertia costali, non conjuncta sed secundæ, prope apicem, venula tiansversa.

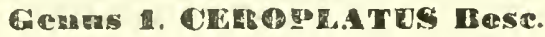

Spec: Typ: Ceroplatus Testaceus Dalm.

3B. Vena longiudinalis prima producta ad summun contra apicem areolæ basilaris. - Vena longitudinalis terti، conjuncta costali non secundæe venula traxscersaria.

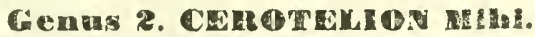

Spec: Typ: Platyuna Laticonnis Mefg. 
AA. Palpi articulis tribus distinctis.

C. Feno longitudinales quinta el sexta non spuria - Vemula transversaria adest inter longitudinales tertian et anteriores.

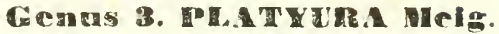

Spec: Typ: Platyura Fasciata Meig.

CC. Veme longitudinales quinta et sexta spurix. - Vemulca llansversaria nulla inter longitudinales tertiam et anteriores.

\section{Genus 4. Mretromin mes}

Spec: Typ: Mycetomia Pallipes Mergl.

Strps LXXX. MACROCERINA Rndn.

Sysox. Famil: Mrcetophlldarc.

A. Ocelli in triangulum disposili. - Tenalomgitudinalis ter-

lia conjuncta septimæ venula obliqua. - Areola analis non adest retro basilarem.

\section{genus 1. machocien meis.}

Spec: Typ: Macrocera Lutea Meig.

A A. Ocelli in lineam subrectam dispositi. - Vona obliqua 
non adest conjungens tertiam et septimam longitum dinales. - Areola analis arlest retro basilarem.

Genus 2. Batertornita befg.

Spec: Typ: Bolitopula Cherea Meig.

Sitips LXXXI. SCIOPHILINA Rnd̆n.

Synow. Famil: Mrcetopullidarur.

^. Alarum venæ longitudinales septem, et si sex tantum, tunc furcas duas venosas constituentes.

B. Inter venas longitudinales primam et secundam, et inter secundam et tertiam venula transversaria aliquia adest.

C. Capul in rostrum satis longum productum.

\section{Genus 1. GNORISTA Hofe.}

Spec: Typ: Gnorista Aplcauls Hofg.

CC. Caput in rostrum longum non productum.

1. Fena longitudinalis prima costalem attingens contra vel ultra originem tertiæ.

5. Tena transversaria unica adest inter secundam longiludinalem et sequentes.

F. Fena prima longitudinalis costali producta circiter con- 
Ira transversariam exterintem. - Vence longitudinatis quartæ conjunctio cum quinta satis interior conjunclione sextæ cum sepplima.

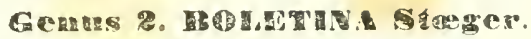

Spec: Typ: Boletwa Parmensis Mihi (nova)

FF. Vena longitudinalis prima costali producta satis ultra transversam cxteriorem-Conjunctio vence longinudinalis quartæ cum quinta magis exterior conjunctione sextæ cum septima.

\section{Gemas 3. Fungha min.}

Spec: Typ: (nova) Fungina Sicula Mihi.

EE. Vemula transversarice duæ adsunt inter longitudinalem secundam et sæquentes.

6. Yena longitudinalis prima distincte producta ultra venulas transversarias retro positas. - Vence longitudinales quarta et quinta conjuncte magis iuterius conjunctione sextæ cum septima.

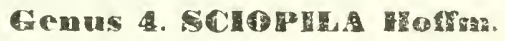

Spe: Typ: Sciopula Lutea Macq.

GG. Sena longitudinalis prima non producta ultra venulas transversarias retro positas. - Vence longitudinales fuarta et quinta conjunctx magis exterius conjunctione sextæe cum septima.

Genus 5. MeOvit wihi.

Spec: Typ: Sciophila Marginata Mgrl. Mgn. 
ii). Lena longitudinalis prima satis brevis et apice satis distans ab origine tertiæ.

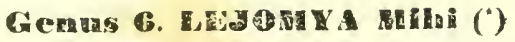

Splec: Typ: Leja Bmaculata Mgn.

IBB. Tence transversarice non adsunt vel inter primam et secundam longitudinales, vel inter secundam et tertiam. 11. Iena longitudinalis quarta ad basim late interrupta et non conjuncta nec tertia, nec quintæ.

\section{Genus z. NuUEATHA Mihi.}

Spcc: Typ: Mycetopula Nesohalis Meig.

111. Fena longiludinalis quarta ad basim non interrupta, ef quintæ vel tertiæ conjuncta.

b. Fena longitudinalis prima non rudimentalis et costalem altingens.

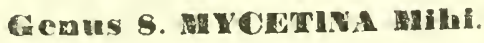

Spec: Typ: Mycetophla Flavipes Macq.

II. T'ma longitudinalis prima rudimentalis, vel vix distinguenda, numquam costali producta.

K. Antemnce duplo et ultra longiores capite. - Palpi articulo primo non crassissimo.

1. Ocelli distincti.

\section{Gentas 9. MCETORHE Feìn.}

Spec: Typ: Nycetopinza Fasciata Meig.

(") Ho cambiato in nome di Leja in quello di Lejomya perche esiste un genere Leja anche nei Coleopteri. 
1L. Ocelli nen manifesti.

Cemus 10. COLEDLA beig.

Spec: Typ: Condyla Fusca Meig.

KK. Antenne parum, et non duplo et ultra longiores capite.

- Palpi articulo primo crassissimo.

Genus 11. PACHPALUU sracq.

Spec: Typ: Pachipalpus Calceatus Milli (nova)

AA. Yence longitudinales alarum sex vel ıninus, et quando sex adsunt furcam venosam unicam conslituunt.

M. Palni articulo primo crassissimo.

Genns 12. HotepaLes whi.

Spec: Typ: (nova) Protepalpus Hirtipes Nihi.

111. Palpi articulo primo crassitie subæquali sequentibus. X. Areola ovata extraordinaria adest in medio vena longitudinalis quintæ.

\section{Genus 13. SIVAPHA Meig.}

Spec: Typ: Sivapua Fasciata Meig.

NN. Areola extraordinaria non adest.

O. V'ence longitudinales sex adsunt margini alarum productæ.

P. Vena longitudinalis quarta, quintæ conjuncta.

Genus 14. Bodithoda whi.

Spec: Typ: (nova) Bolithobia Lateralis Mihi.

Pl. Vena longitudinalis quarta tertix conjuncta. 
Q. Vena transversaria aliqua adest inter primam el secuim dam longitudinales.

\section{Genus 15. MYCETOMYZA Migh.}

Spec: Typ: (nora) Mycetomyza Sciarina Mili.

QQ. Iena transversaria nulla adest inter primam et secundam longitudinales,

\section{Genus 16. Ma Crovedra Maeq.}

Spec: Typ: Macroneura Winthemil Macq.

00. Vence longitudinales quinque tantum margini alarum productæ.

\section{Genus 1\%. BoLTHomyza Mihi.}

Spec: Typ: (nova) Bolithonyza Spqulla Mihii

Stirps LXXXII. SCIARINA Rndn.

T: Lugubres p. Meig. - t: Fux́gicole p. Latr. Maci.

- mycetophilide p. Macal. Bigot. - cecidonybes p. Westiw. - sciarin.: Zell.

Unicum dienus 1. SCRARA Meler.

Spec: Typ: Thpula 'home Lin. 
398

Stuns LXXXII. HCROMYMA Rndn.

uestremina Radn. - Cælep Synon: Famil. CachopRYDARUM.

A. Vene longitudinales alarum sex, spuriis etiam consideratis: duabus intermediis furcam venosam conslitientibus.

§. Antenne maris articulis petiolatis quindecim vel sexdecin.

C. Vena longitudinalis secunda costalem allingens prope apicem, aut satis ultra medium alarum. - Tarsi nom dilatati.

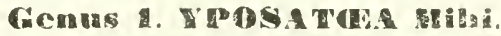

$$
\text { Cienera conjincta }\left\{\begin{array}{l}
\text { Lestrema Macq. } \\
\text { Zigoneuna Meigr. } \\
\text { Mhoosciara Rindu. }
\end{array}\right.
$$

CC. Vena longitudinalis secunda costalem attingens circiter ad medium alarum. - Tarsi basi dilatali.

\section{Gerne Cacocha mallo.}

Spec: Typ: Catocha Latipes Halid.

BB. Antenne maris articulis novem Lantum instrutte.

\section{Genus 3. ANA Hewe Ellid.}

Spec: Typ: Anarete Candidata Halid.

AA. Tence longiludinales alarum quinque tantum, quia lermedia spuria non furcala.

b. Anterno maris articulis decem non distinctissime petion latis. - Fuminæ articulis sex sessilibus.

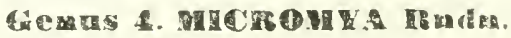

Spec: Typ: Hicronya Lecorvar Rndu. 
DD. Antemo maris articulis quatordecim vel quindecin:

- Fomina circiter duodecim instructæ.

E. Antenna articulis non manifeste petiolatis etiam in mare.

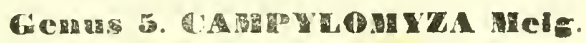

Spec: Typ: Canpylomyza [ilavipes Meig.

EE. Antenne maris articulis distinctissime petiolatis.

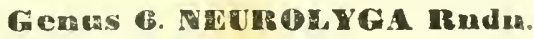

Spec: Typ: Nevrolyga Fenestralis Rndn.

StIRPS LXXXIV. CECIDOMYNA Rndn.

Srnon: Famil: Cecidomydaru.

A. Fonce longitudinales alarum quatuor distinctæ.

b. Fence longiludinales prima el secunda venula obliqua conjunctie.

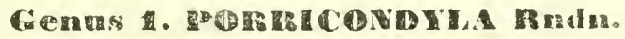

Spec: Typ: Cecidoyza Azbitarsis Meig.

BB. Inter venas longitudinales, venula nulla obliqua.

C. Vena longitudinalis prima marginem altingens suh anicem alarum.

D. Anteme maris articulis satis manifeste petiolatis.

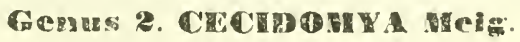

Spec: Typ: Cecionmya Producta Meig.

DD. Antenna in utroque sexu articulis nom manifeste petiolatis E. Antennx etian in mare articulis cylindrico-elongalis.

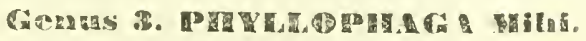

Spec: Typ: Cecroonsa Fuca Meig. 
EE. Anterna articulis subglobulosis in mare et fnemind, vel in formina sub-ovati.

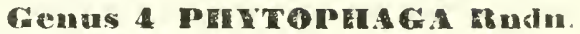

Spec: Typ: Phytopaga Cerealis Rudn.

CC. Vena longitudinalis prima satis ante apicem alarum margini antico producta.

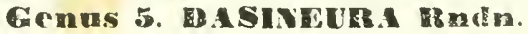

Spec: Typ: Dasineura Obscura Rndn.

Ad. Vence longitudinales alarum tres, vel raro duæ la:lum, prima considerata étiamsi cun cosiali subconfusd. Antennoe articulis non petiolatis in utroque sexil.

F. Ala venis tribus longitudinalibus, prima aliquando cun costali subconfusa.

1. Thorax in collum cylindricum non productus

\section{Genus 6. LASHOPTES Sheig.}

GG. Thorax in collum cylindricum, atteniratum, prouhietus.

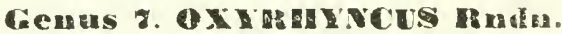

Spec: Typ: Oxyrhyngus Longigollis Rndn.

FF. Alte venis duabus tantum brevibus et parum distinetis.

Genus 8. Hachenerba Rudm.

Spec. Typ: Buacrinecra Fuscogrisea Rndu.

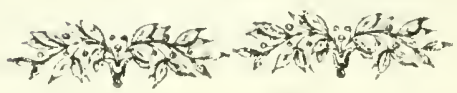




\section{GENERUM STIRPIUM ET FAMILIARUM}

\section{INDEX}

Acrinya Desv. . . . . . . . . Pag. 73

Achanthipodus Radn. . . . . . . . . . " 144

Achanthiptera Findn. . . . . . . . . . 11 93

Achantholena Pndn. . . . . . . . . . 101

Acinia Desr. . . . . . . . . . . I1 113

Aciura Desv. . . . . . . . . . . . " 113

Aclia Desv. . . . . . . . . . . " " 60

Acrocera Mrgn. . . . . . . . . . . ॰ 166

Actora M!gn. . . . . . . . . . . . " 10 '

Acules Hoffg . . . . . . . . . . . . 4 177

Agrumyza Fall. . . . . . . . . . . . \& 121

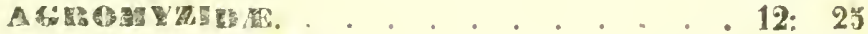

AGROMZINd. . . . . . . . . . . . 25: 120

Albertia Rndı. . . . . . . . . . . . 11 77

Alloneura Rndn.. . . . . . . . . . . 140

Alophora Desv. . . . . . . . . . e 81

Alophroida Rndn. . . . . . . . . (f 188

Alticomerus Radn. . . . . . . . . . . . . 121

Anarete Halid. . . . . . . . . . . " 198

Andrenosoma Rudn. . . . . . . . . If 160

Anisomera Mgn. . . . . . . . . . . . " 184

Anopheles Mgn. . . . . . . . . . . . " 170 
Anoplomerus firlin........... Pag. 14t morthus Stogr. . . . . . . . . . \& 145 Anthomya kign. . . . . . . . . . . . " 95

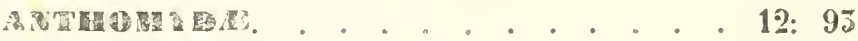
Antuonina . . . . . . . . . . 24: 23 Anlliracomya Rot!n. . . . . . . . . " 87 Antbrax Scop. . . . . . . . . . . 163 Aphria Desv. . . : . . . . . . . . 4 73 Aphritis Latr. Apogon Rindn. ॥ 5 ' a $17 \%$ Aplerina Macq. , . . . . . . . . . 4 125

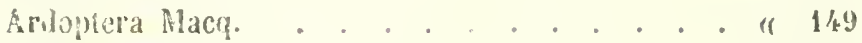
Argyea Macq. . . . . . . . . . . . $\pi 141$ Amyiopila Rnde. . . . . . . . . . \& 162 Alicia Macq. . . . . . . . . . . . . a 95 Ascra Megrl. . . . . . . . . . . . " 49

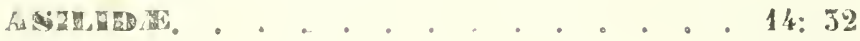
ASILINE. . . . . . . . . . . 32: 152 Asilus Lin. Aspistes IIofrm. Astein Mga. AST INA . Asllenia Westiv.

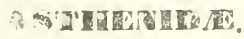
(1 159 (1 17\% - 135 27: 135 ir 190 17: 39 ST UENINA 39: 190 Fotinella Duf. A STOMELLNA a 166 ceneura Macq 3': 166 Atserigona !ndn. (I 140 97 
A therix Mgn.

Bacca Fabr.

Beriulva.

Beris Latr. .

Bertéa Rodn.

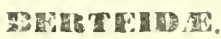

Berteina.

Bibio rabr.

Bigonicheta Rndn.

ER:

56: 175

Biblonisa

Biomya Riudn.

" 173

(" 190

17: 39

39: 180

blepharidea solu.

(1) 174

Blepharigena Rocin.

Blepharipa Rndn .

Boletina Stæg.

Botihobia Rnia.

Bulilhonyza Rum

61

Bolithophila Hofg.

둔.

16: 36

56: 173

Bombrija .

72

67

69

$+771$

\& 194

- 136

- 197

- 193

14: 53

33: 162

Bombylisoma Rndn

a $16 \%$

Bumbylius Lin.

a $16 \%$

Bophrosia Renu

18.3

Bothria Roda.

68

Brachicoma Mgn.

69

Brachiglossum Radn.

- 36. 
Brachipalpus Hacq. . . . . . . Pag. 48

Brachineura RnG̊n. . . . . . . . . . . 200

Brachiopa Hofg. . . . . . . . . . 52

Brachistoma Mgn. . . . . . . . . 150

Callicera Mgu. . . . . . . . . . . ๖ร

Chllicerina. . . . . . . . . . 20: 85

Calliphora Desv. . . . . . . . . 90

Calliprobola Rndn. . . . . . . . . \& 47

Callomya Fall. . . . . . . . . . . \& $\$ 38$

Calobata Mgn. . . . . . . . . . a 116

Camarota Mgn. . . . . . . . . . . 120

Campreneura Rnda . . . . . . . . . 52

Campregaster Rndo . . . . . . . . . 80

Campylonyza Mgn. . . . . . . . . . . 199

Caricna Desv . . . . . . . . . . . . . 98

Carpomya Radn . . . . . . . . . \& 111

Calocha Filid. . . . . . . . . . " 198

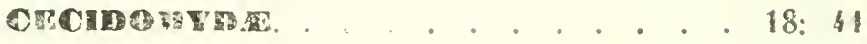

C.ecidomysa . . . . . . . . . . 41:199

Cecidomya Latr. . . . . . . . . . . 199

Cephalemya Clark. . . . . . . . . . . 45

Cephalia Mgn . . . . . . . . . . . e $11 \mathrm{~b}$

Cephenemya Lall. . . . . . . . . . 4 4

Cerajocera Rndu. . . . . . . . . \& 111

Ceratitis M. Leay. . . . . . . . . " 114

Ceratopogon Mign. . . . . . . . . . . . 176

Ceraturgus Widm . . . . . . . a 156

Ceria Fabr. . . . . . . . . . . \& 55

Ceroctena Rndn . . . . . . . " 186 
Ceromasia Rndn Pag. $7 i$ Ceroplatima.

Ceroplatus Bosc

- 19!

Ceroptera Macq

- 12!

Cerotelion Rndn

- 19 !

Cheilopogon Rndn.

- 157

Cheilosia Mgrl.

- 51

Chelisia Rndn.

- 10 ?

Chemalida Rndn

- 180

Chersodromya Ifalid.

Chetina Rndn.

- 147

- 85

Chetogena Rndn . . . . . . . . . . . . . 68

Chetoliga Rndn. . . . . . . . . . . . . 66

Chetostoma Rndn. . . . . . . . . . . . . 112

Chiliza Mgn . . . . . . . . . . . 123

Chlzuzina. . . . . . . . . 85: 122

Chionea Dalm. . . . . . . . . . 18?

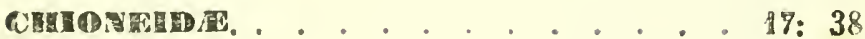

Chonetala . . . . . . . . . . . . . $58: 189$

Chiroman!is Rndn. . . . . . . . . . 148

Chiromya Des\%. . . . . . . . . . . . . \& 121

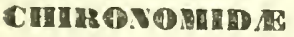

15: $3 i$

Chironomisa

57: $17 \%$

Chironomus Mgn .

Chirosia Rndn.

- 176

- 102

Culoropina

26: 125

Chlorops Mgn.

Chorisops Rndn.

- 123

Chorthophila Macq

173

Chryorhina Hefg.

- 96 
Chrysollidmys Touln.

Chrysogazer $\mathrm{H}_{\mathrm{g}}$.

Chrysomya hiacu.

CuRYSORYXA

Chrysopivia Mucq .

Chrysops Mgn.

Chrysotoxum Mgn.

Chrysolus Mgn.

Cirillia Findn.

Cistogaster Latr

Clairvillia Desv

Clausicella Rndn.

Cleigastra Masq .

Clinocera Mgn.

Clisı Mgn.

Clorisoma Rndn

Clytia Desv.

Cnemopogon Rndu.

Cnephalia findu.

Cœiopa M̂gn.

Conia Desv.

Conomya Latr.

$$
\begin{aligned}
& \text { Pas. } 38 \\
& \text { a } 5 \\
& \text { - } 163 \\
& \text { 5? } 167
\end{aligned}
$$

. 1

7

(i)

10 !

$1: 2$

76

168

$8 \cdot 3$

« 100

" 6?

. $10 \mathrm{~J}$

" 150

" 172

Conomyns . . : . . . . . . 36:172

Conosia Mgn. . . . . . . . . ... . . " 97

Conicera $H_{\mathrm{gn}}$. . . . . . . . . . . . " 139

COM

Connpilla Rindn . . . . . . . . . . " 56

Coropint . . . . . . . . . 21: 56

Conops Lin. . . . . . . . . " 4 . 
Copromyza Fall.

Coprourzina

Corethra Mgu .

Corethrina.

Cordyla Mgn.

(1 $17 \%$

Cordylura Mgn.

37: 177

Coremacera Rndn.

Ctenoceria Rndi.

Ctenophora Fatr.

Ctenulus Rudn.

Culex Lin.

Culicina.

Cyclogaster Macq.

(1 196

(1 10,

II 16ij

- $18 \%$

a 187

Cylindrosoma Rudn.

\& $10 ?$

(1) $17 \%$

Cylindrotoma Maca.

37: 178

Cyllenia Latr .

Cynomya Desv

* 168

(1) 79

(1 181

(1 16́

Cyphocera Maca.

CYETER

6.3

Crrtina

1': 35

Cyrtoma Mgn .

35: 165

Cyrtoneura Macq. . . . . . . . . . . . " 91

Cyrtophloba Rndn . . . . . . . . . 68

Eyrtopogon Lò̀w. . . . . . . . . " "

Cyrtesia Perris. . . . . . . . . . " 165

Cyrtus Latr . . . . . . . . . " " 163

Dactiliscus Rndn. . . . . . . . . " 158

Dacus Mgn. 
208

Dalmanuia Desv.

Iig. 33

I)asiops Rndn.

120

7asipngou Fabr.

1) ASIPOGONINA.

Dasineura Rudn

Dasiphora Desv.

20)

DeGeeria Mgn.

Dexia Mgn.

Deximorpha Rndn . . . . . . . . . . " 8 先

Dexina . : . . . . . . . . . . . 25: 8.3

Dexiosoma Rudn. . . . . . . . . . \& $8 \%$

Tialineura Rndo

Diaphorus Mgn.

Diastata Mgn .

Dichela Mgn. .

Dictenidia Brulle

Didea Macr.

Dilophus Mgn.

Dinera Desv

177

Dioctria Fabr

Discomyza Mgn.

Dithricha Rndn

Dixa Mgn.

Dolicopeza Mgn. H OLICD P R

DOLICOPINA .

Domomyza Rndn. 144 Doricera Mga. 
Doros Mon. . . . . . . . . . . Pay. 50

Drapetis Mgn . . . . . . . . . . . " 147

Drimeja Mgn . . . . . . . . . . . . . " 9 "

Driodromya Rndn. . . . . . . . . . \& 150

Drosophila Fall. . . . . . . . . . . " 15̃

Drosopmlina . . . . . . . . . 27: 155

Dryomyza Fall. . . . . . . . . \& 105

Echinomya Dmrl . . . . . . . . . . " 6 .

Elachiptera Macq. . . . . . . . . . . 18 12\%

Elaphropeza Macq. . . . . . . . . . . " 147

Elasinocera Rndn. . . . . . . . . . . . " $\$$. .9

Elmoplaila Rndn . . . . . . . . . . . " $1 \% 2$

f

Elgiva Mgrl. . . . . . . . . . . a 100

Fliozeta Pindn. . . . . . . . . . . " 82

Elomya Des. . . . . . . . . . . \& 81

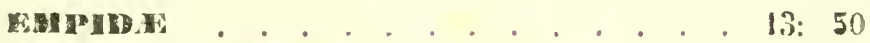

Empidina . . . . . . . . . . 30:149

Empis Mgn. . . . . . . . . . . . \# 151

Enicita Westw . . . . . . . . . . 117

Ephiała Fall. . . . . . . . . . . 131

Ephidriva . . . . . . . . . 26:12.)

Kphippium Lâr. . . . . . . . . . . . " 169

Fpidesmia Rndn . . . . . . . . . . " 112

Erebia Dest . . . . . . . . . " " 6 . .

Erinnia Desv . . . . . . . . . . . " $7 \tau$

Erioptera Mgn. . . . . . . . . . . . 4179

Fistalina......... . . . 20: 4́ 
20

Wristalis Fabr.

Pas. 4:

Eumerus Mgn. . . . . . . . . . . . . " 48

Eurina $\operatorname{Mgn}$. . . . . . . . . . " " 120

Exaloma Mgn . . . . . . . . . . . " 170

Exodontha Bellardi . . . . . . . . . " 169

Exogaster Prún . . . . . . . . " 78

Exoprosopa Macr. . . . . . . . . " 162

Ezorista Mgn . . . . . . . . . . . " 67

Fallenia Mgn. . . . . . . . . . " 161

Fallentad . . . . . . . . 35: 161

Fisceria Desv . . . . . . . . . . . " 73

Frerea Desv. . . . . . . . . . . . " 81

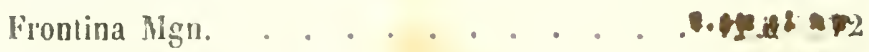

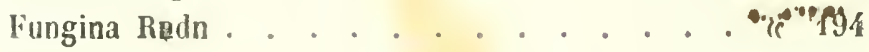

Custrichelins Rnen. . . . . . . . " 156

Ledia Mgn . . . . . . . . . " 77

Geomyza Fall . . . . . . . . " 118

Germaria Desv. . . . . . . . . . " 62

Geron Hoffm. . . . . . . . . . . " 163

Gitona Mgn . . . . . . . . " 13 \%

Glabrinus Rndn. . . . . . . . . . \& 152

Glochina Mgn . . . . . . . . . . " 185

Gloma Mign . . . . . . . . . " 149

Glossigona Rndn . . . . . . . . . " 58

Glossista Rndn . . . . . . . . " 16.5

Gnoriste Hoffg. . . . . . . . . . . . " 195

Gonatherus Rndn. . . . . . . . . . " 99

Gonia Mgn. . . . . . . . . . . . . . " 62 
Gonigiosstim Rudn. . . . . . . . Pdğ 110 Gonipes Latr . . . . . . . . . . " 138 Gunirhyneus lindn. . . . . . . . . . . " 58 Graphomya best . . . . . . . . . . . " 91 Gymnocheta [les\%. . . . . . . . . . . " 64 Gyniopha Fall. . . . . . . . . . " 198

Gymnophora Macq. . . . . . . . . . . . . 157 Gymnosoma $\mathrm{Hgn}$. . . . . . . . . . " 81

Halidaya Rndn. . . . . . . . . . \& 117 Haltericerus kisdis. . . . . . . . . a 143

Hlamnobdtes Stanı. . . . . . . . . . 1/e

Ularrisia Mgn . . . . . . . . . . . . . . 6.5

Hecamele Curt. . . . . . . . . . \& 151

Ilelomyza Fall. . . . . . . . . . . . " 109

Ilelophihus Mgn . . . . . . . . . " 45

Ilemerodromya lisfo. . . . . . . . . 148

Henoridina . . . . . . . . . 38160

Henops Fall. . . . . . . . . . . . . " 166

Herina Desv . . . . . . . . . . 109

Ileringia Rndn. . . . . . . . . . \& 55

Ileteromyza Fall . . . . . . . . . \& 103

Heteroptera Macq. . . . . . . . . . . . \& 124

licteropterina Mace. . . . . . . . . . \& 70

Heterostoma Rnln . . . . . . . . . . . a $10 \%$

liliara Nign. . . . . . . . . . . . • 151

Hilarella Rndn. . . . . . . . . . . . . \& 70

Hirmoneura Wietm. . . . . . . . . . 162

Ilematobia Desr . . . . . . . . . . . . . 9

Ilæmatopota Mgn. . . . . . . . . " $\$ 70$ 
Evbotidina Pag. 50: is

Hybos Fabr.

Hydrellia Desv.

- 1 is

IIydrophoria Macq.

Hydrophorus Fali.

Hydroloca Dest.

IIylara Mgn

Hylemya Desv.

Hypoderna Clark.

Hypoderyixa

Hypostena Mgn.

Ibisia Rndn.

Idia Mgn.

$11 \quad 154$

Idioptera Macq.

llisia Rnun.

Hisomya Rndn.

llisophila Rndn.

Istoglossa Rudn.

- 182

- 180

- 180

$7 \%$

Labidigaster Macq

1176

Laphria Fabr . . . . . . . . . . « 160

LAPHRINA.

Iasiophthicus Rndn.

Lasiopogon Lòèw.

a 156

Lasiops Mgn

- 96

I.asioptera Mgn. .

- 200

Lejomya Rndn. (Leja Mgr?) .

- 193

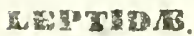


LEPTIDINA

Leptis Fabr.

Leria Desv .

Lestremya Hacq

Leucopis Mgn.

Limncea Rnàn

- 15.4

- 103

Limnobia Mgn.

LIMNobINA

198

- 120

- 181

Limnomyza Rndn.

Limnophila Maca.

Limnophora Desf

Limosina Macy

185

179

Lionenra Rndn.

Lissa Meig .

Lispe Latr .

Litorella Rndn.

Lozatia Mgn .

Lenchea Fall

185

- 183

- 97

- 124

- 179

- 110

- 9.5

* 101

- 163

ब 119

Loncueina .

25118

Lenceptera Mgn.

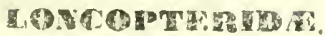

28137

I.CXCOPTERINA

13: 28

L.pplosia Mgn.

1.oxocera Fabr.

Lucilia Desv

Lucina Macq.

Lyperosia Pinda

Macherium Hald. 
Hacrocera Mgn.

Macrocerind

Macrodichon Ruth

Mlacropeza Mgn.

Macroneura Macr.

Maliza Fall.

Alallota Mgu.

Manliseza Rudn

Masicela Mgn.

Medeterus Latr

Medorilla Rnin.

Megachetum Rndn.

Megaselia Rndn.

Megerlea Desv.

Nelania Mgn .

118

Melanomya Rndn.

Melanopliora Mgn

Melia Desv.

Melieria Desv.

Mekelia Des

38

Meoneura Rum.

Merodon Lair.

Meromyza Mign.

Mesembrina Mgn.

Melopia Mgn

Ricromya Radn. 
Micronuna

Micropalpus Macq.

Micropeza Mgn.

Microplorus Macn

116

Milesia Lat

Milichia Mgn .

Mittogramma Mg!n.

151

Mimosciara Rnón.

Mlintho Desv. .

Morphomya Rndu

Morinia Desy .

47

120

Mulio Latr.

Musca Lin

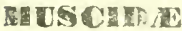

Muscina

12: 21

Mya Rindn

25: 89

Myantha Rindn.

Myathropa Rindn

Mycetina Rindn

Mycetobia Mgn.

Mycetemyza Rrian

Hycetophila Mgn.

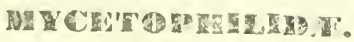

Nycomya Rndn

hycopliaga Rndn.

Mydas Fabr.

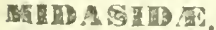

Nydastiva.

95

- 40

- 193

192

- 197

a 195

18: 40

a 194

- 102

Myobia Desv.

32161

II $\%$ 
216

Myodina Desq.

Myoleja Rndn.

Myopa Fabr. .

Myopina . . . . . . . . . . 21: 57

Myopina Desv . . . . . . . . . . . " 99

Myopites Brebis. . . . . . . . . . . " 110

Myostoma Desv . . . . . . . . . ж 83

Myospila Rndn. . . . . . . . . . . " 91

Napra Desv . . . . . . . . . . " 150

Nematocera Mgn. . . . . . . . . . " 18 \%

Nemestrina Latr . . . . . . . . . . " " $|6|$

Nemopoda Hacq . . . . . . . . " " 117

Nemocera Desv. . . . . . . . . . " $6 \%$

Nemorilla Rndn . . . . . . . . . . " 66

Nemorius Rndn. . . . . . . . . . . " 171

Nemotelus Geofr . . . . . . . . . " 167

Nephrocerus Zett. . . . . . . . . " 159

Nephrotomat Mgn. . . . . . . . . . " 187

Neurátelia Rndn . . . . . . . . . . " 19;

Neurigona Rndn. . . . . . . . . . . " 142

Neurolyga Raun . . . . . . . . . . " 199

Norellia Desv . . . . . . . . . " 10 .

Noterophila Rndn. . . . . . . . . . " 153

Notiphila Fall. . . . . . . . . . " " 189

Nyclia Desv. . . . . . . . . . . . " 86

Ochtera Latr. . . . . . . . . . . . " 132

Ochtiphila Fall. . . . . . . . " 118

Ocyptera Latr. . . . . . . . . is 78 
Ocypterula Rnin. . . . . . . . . Pag. 78 Odonhliocera Haç. . . . . . . . . . 4 127

Odontomya Latr. . . . . . . . . . . 1170 Ouonthonyna . . . . . . . . . . 35169 Gidalea Mign. . . . . . . . . . . . . " 152 COSTERTE . . . . . . . . . . . 10: 19 Estrina. . . . . . . . . . 19: 42 CEstrus Lin. . . . . . . . . . . . . If 42 Olivieria Desv . . . . . . . . . . If 78 Onesia Desv. . . . . . . . . . . a 90 Onodontha Rndr. . . . . . . . . \& 9 \& Opelia Mgn. . . . . . . . . . . . a 138 Opztina. . . . . . . . . . 28158 Ophira Desv.. . . . . . . . . . . \& 94 Oplocheła Pindn . . . . . . . . . . . 113 Oplogaster Radn. . . . . . . . . . . \& 98 Opomyza Fall. . . . . . . . . . . 119 Oregocera Rndn . . . . . . . . . . . 106 Orellia Desv . . . . . . . . . . \& 113 Ormosia Rndn. . . . . . . . . . . 180 Orosmya Rndn. . . . . . . . . . . « 182 Orphnephila IIalid . . . . . . . . . a 189

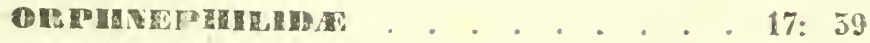
Orphephillisa. . . . . . . . . . . . 59189 Ortalidina . . . . . . . . . . 24 108 Or! lis Fall. . . . . . . . . . . . \& 108 Orthochile Latr . . . . . . . . . . . \& 14k Orthoneura Macq. . . . . . . . . . . . « эั Drygma Mgn . . . . . . . . . c 10 出 
218

Cscinta. . . . . . . . . Pag. 26127

Oscinis Latr . . . . . . . . . . 128

Otites Latr. . . . . . . . . . . . 109

Oxycera Mggn. . . . . . . . . . . . " 16 ?

Oxina Desv. . . . . . . . . . . . . 110

Oxgrhynchus Radn . . . . . . . . . 200

Pachỹgaster Mgn. . . . . . . . . . 167

Pachylomera Rudia. . . . . . . . . "122

Pachymeria Sieph . . . . . . . . " 131

Pachypalpus Macd . . . . . . . . . 196

Pachyrhyna Macq. . . . . . . . . . . 188

Pacutstonima . . . . . . . . . . 5145

Pachyslomes Latr. . . . . . . . . . 155

Palloptera Fall. . . . . . . . . . . . 119

Palpibraca Roin. . . . . . . . . 63

Salpimega Ruda. . . . . . . . . a 13 6

Pangonia Latr. . . . . . . . . . . 171

Pangonira . . . . . . . . . . . 35

Paragus Latr . . . . . . . . . . . " 5 k

Eedicia Latr . . . . . . . . . c 187

Pegophila Rntin . . . . . . . . . . " 129

Pelecocera Hofg . . . . . . . . . " 50

Pelidnopera Rindn. . . . . . . . . . 107

Pelosia Rudn . . . . . . . . . « 185

Pentelluria Mgu . . . . . . . . « 175

Peralochelus Rndn . . . . . . . \& 419

Pelagnia Rindn . . . . . . . . . . " 61

Plania Mgn . . . . . . . . . . " " 7 \&

Phanissoma Rixn . . . . . . . . " it 
Phasia Mgn . . . . . . . . . . Pag. 8: Prashina . . . . . . . . . . . . 22: 813

Philammosius Fndn . . . . . . . . . . " 15t Philodendria findn . . . . . . . . . . . " $15 \%$

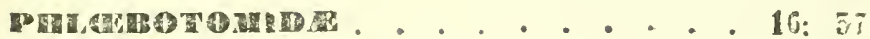
Phloebotomina . . . . . . . . . . 5717

Phlnebotomus Rndn . . . . . . . . . . a 178 Phora Latr . . . . . . . . . . . . . " 157

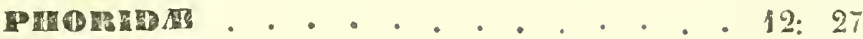
Pliokina - . . . . . . . . . . . 27:136

Phorocera Mgn . . . . . . . . . . . . 67 Phorostoma Desv . . . . . . . . . . . . If 85 Phoroxypha Rndre. . . . . . . . . a 140 Phrosia Desv . . . . . . . . . . \& 100 Phthyria Mgn . . . . . . . . . . . . . 11163 Phyllodromya Zett . . . . . . . . . a 148 Purllodronya . . . . . . . . . . 30147 Phyllomyza Fall . . . . . . . . . * 127 Pliyllophaga Rndn . . . . . . . . . \& 199 Phyto Desv . . . . . . . . . . . . 73 Phylomyptera Rndn . . . . . . . . . . a 61 Phytomyza Fall . . . . . . . . . - a 135 Phylophaga Rndn . . . . . . . . • . * 200 Piophila Fall. . . . . . . . . . . . 118 Piothepalpus Rndn . . . . . . . . . . a 196 Pipiza Fall . . . . . . . . . . . , 53 Pipizella Rndu . . . . . . . . . . . \$

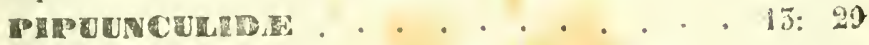
Pnunculina . . . . . . . . . . . 29139 
Pipunculus Latr

Pag. 140 Plagia Myn . . . . . . . . . . . . . \& 69

Platychira Rndn . . . . . . . . . . \& 64

Platynochetus Wiedm . . . . . . . \& 4 š

Platycephala Fall . . . . . . . . . \& 126

Platypeza Fabr . . . . . . . . . . . 139

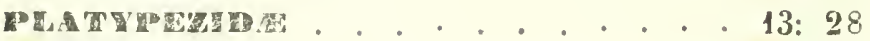

Platypezlimi . . . . . . . . . 28: 158

Platystila Macq. . . . . . . . . . . a 122

Plalystoma Latr . . . . . . . . . . . 109

Platylelma Rndn . . . . . . . . . . . 138

Platyura Mgn . . . . . . . . . . 192

Plaxemya Desv . : . . . . . . . . . " 99

Ploas Latr . . . . . . . . . " 164

Pogonosoma Rudn . . . . . . . . . " 160

Policheta Rindn . . . . . . . . . . . 67

Polionoma Rndn . . . . . . . . . 107

Pollenia Desv . . . . . . . . . . . . a 90

Porphirops IIgn . . . . . . . . . . . 145

Porriondyla Padn . . . . . . . . 199

Proboscina Rndn . . . . . . . . . . . " 88

Prosena S. F. S. . . . . . . . . $\pi$ 84

Prothecus !ndn . . . . . . . . . " 15 "

Psalida Rndn. . . . . . . . . . . . 76

Psarus Fall. . . . . . . . . . . 5 . $^{\prime}$

Psicoda Latr . . . . . . . . . 178

Psicodisa . . . . . . . . 38: 178

Psila Meigr . . . . . . . . . . . . 122

Psilopa Fall. . . . . . . . . 150 
Psilopus Mgrl

Psilota Mgn

Pterelachisus Rndn.

Pteremis Rndn.

Pterospilus Rndn.

Pticoptera Mgn

Ptilocera Desv.

(1 14\%

Pycnopogon Lòèw

Pyrellia Desv

Pyrrosia Rndn

53

- 188

- 124

- 152

\& 187

(1 87

- 157

1 90

73

Ragheneura Rndn

Ramphidia Ngु" .

Ramphina Macq.

144

Ramphomya Hof $\mathrm{g}$.

Rapuina .

181

Raphium Mgn

77

- 131

29: 145

Rhyucomya Desv

\& 146

Rhynchosia Macq

74

MUIY'PIIDAE .

73

RHypilisa

18: 39

39: 191

Rhyngia Scop

52

Rhyphus Latr

191

Ripidia Mgn .

Rivellia Desv .

186

Roeselia Desv

111

72

Saccopterix Halid

179

Saltella Desv .

- 179

Sarcophaga Mgn

117

s6i 
Sarcophila Rndn Sargus Fabr. Scatina Desv. Scatophaga Mgn Sgatophagina $8 i$ 168 * 102 a 102 ㄴ) 98 Scalopse Desv 174 Scatopsina ofis: 174 Sciara Mgn - 197 Sciarina 10197 SCEYOPRNT U:

Saenopinixa 54: 167

Scenopinus Latr - 167 Sciomyza Fall 107 Scromyzina . เ) 104 Sciophila Hofg. 194 Sciopuilina. 40: 195 Scopolia Desv Sepedon Latr Sepsis Fall . $10 \%$ Sericomya Mgn Serromya Halid 116 Sibistroma Mgn Simplesta Mgn Sinapha Mgn .

Siphona Mgn . 143 Siphonina

Simulia Mgn

Simuliva . 
Spania $\mathrm{Hgn}$. . . . . . . . . . . Pag. " 154

Spanina. . . . . . . . . . . J1 154

Spathulina Rondn . . . . . . . . . « 113

Spazigaster Rndn . . . . . . . . . . . \& 49

Spaziphora Rndn. . . . . . . . . . . . \& 99

Sphærocera Latr. . . . . . . . . . c 12/

Spærophoria S. F. S. . . . . . . . \& 50

Sphegina Mgn. . . . . . . . . . . . \& 49

Sphyxæa Rndn . . . . . . . . . . . " 16

Sphyximorpha Rndn . . . . . . . . . \& 55

Sphyxosoma Rndn . . . . . . . . . . \& 56

Spilogaster Macq. . . . . . . . . . . . « 97

Spiloptera Rndn . . . . . . . . . \& 181

Spilosia Rndn . . . . . . . . . . . \& 66

Sticgeria Rndn . . . . . . . . . . . a 99

Stegana Mgn . . . . . . . . . . . . " 153

Stenopogon Lóèw . . . . . . . . " " 158

Stevenia Desv.. . . . . . . . . . . . " 80

Stomoxidna. . . . . . . . . . . . 25: 92

Stomoxis Geofr . . . . . . . . . " " 92

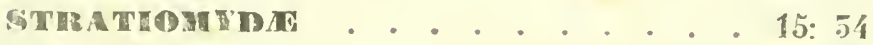

Stratiomys Geofr . . . . . . . . . \& 169

Subula Mgrl . . . . . . . . . . . . . " 172

Sycorax IIalid

ETCORAXIDE

Sycoraxina

Vide nota Familiæ

- 17

Syllegeptera Rindn

96

Sylvius Mgn . . . . . . . . . . II $17 \mathrm{i}$

Syritta S. F. S. . . . . . . . . " " 48 


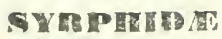

Pag. 11: 19

Syapuina

20: 16

Syrphus Fabr.

TA DANOBDA

15: 35

Tabanina

35: 170

Tabanus Lin

TACUIDROATYNa

50: 146

Tachidromya Mgn

(" 146

Tachina Fabr

TACUININA

22. $\quad 39$

Tachipeza Mgn

Tanipeza rall .

- 115

Tanpezina

2411 th

Tanipoda Rudn

" 116

Tanipus Mggn .

Taphrophila Rndn

Taphrosia Rndn

Teicomyza Macq .

Tephritis Latr

Terel!ia Desv

Terelliosoma Rndn

Tetanocera Dumer

Tetanops Fall .

(1 176

(1 185

" 182

" 1,30

(1) 112

" 11/

109

(1) 106

(1 109

Thecophora Rndn

Thelaira Desv

Themira Desv

117

Thereva Latr

$15:$

TuEREVINa.

51: 135 
Therig Desr . . . . . . . . . . " 8.5

Therina Mgn . . . . . . . . . . . 121

Thryplocera Hacq . . . . . . . . . . . . 60

Thryptocheta Rudn . . . . . . . . . . 154

Thyreophora Latr . . . . . . . . . . . , 123

l'imia Mgn . . . . . . . . . . . . . 115

Tipula Lin. . . . . . . . . . . . . 188

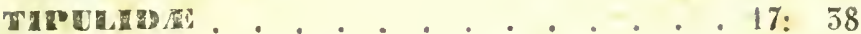

Tipulins . . . . . . . . . 38186

Toxopora Widm . . . . . . . . $16 \AA$

Trichina Mgn . . . . . . . . . \& 152

Tricocera Mgn . . . . . . . . . . . (1 183

Tricogenia Rndn . . . . . . . . . . 88

Tricoliga Rndn . . . . . . . . . . . 1i甲

Tricopalpus Rndn . . . . . . . . . . \& 100

Tricopeza Rodn . . . . . . . . . . 150

Tricops Pindn. . . . . . . . . . . 96

T'riglyphus Lò̀̀w . . . . . . . . . . . . 55

Trimerina Macq . . . . . . . . . . . " 132

Triphløba Rndn . . . . . . . . . . 136

Trixa Mgn. . . . . . . . . . . . 70

Trixoscelis Rndn. . . . . . . . . . . 13k

Trixostomus Rndn . . . . . . . . . . 150

Tromodesia Findn . . . . . . . . . . . 87

Tropidia Mgn . . . . . . . . . . . . 40

Trupanea Macq . . . . . . . . . 159

Jla Halid . . . . . . . . . . . 185

Ulidia Mgn . . . . . . . . . . . 115 
Uromya $\operatorname{Mgn}$. . . . . . . . . . . . 75

Urophora Desv . . . . . . . . . « 110 Usia Lair . . . . . . . . . . . . 165

Vermileo Macq . . . . . . . . . . . 155

Volucella Gofi . . . . . . . . . . a 44

Volugelliva . . . . . . . . . . . 20: 43

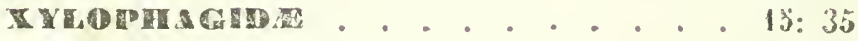

Xylophagina . . . . . . . . . . . 36: 172

Xylophagus Mgn. . . . . . . . . . * 172

Xylota Mgn, . . . . . . . . . . . u 48

Xyloteja Rndn. . . . . . . . . . . \& 48

Xyphura Brullé . . . . . . . . . . 187

Xysta Mgn . . . . . . . . . . . \& 82

Zelterstedtia Rndn . . . . . . . . . c 14

Zeuxia Mlgn . . . . . . . . . . . . . × 85

Zigoneura Mgn . . . . : . . . . . . . " 198

Zodion Latr . . . . . . . . . . . . is 57

Zodonina . . . . . . . . . 21: 57

Yposatca Rndn . . . . . . . . . . 198

Wiedmannia Zelt. . . . . . . . . \& 150 


\title{
NOMI DECHI ADTORI
}

\author{
CITATI NELL'OPERA
}

\section{COLLE ABBREYIATURE CORRISPONDENT}

Bellardi - Bell.

Bigot

Bosc

Brebisson - Breb.

Brente (De) Breme

Bremi

Brullé

Cirillo

Clark - Clk.

Clug.

Contarini - Contar.

Costa Achille - Costa A.

Cosia Oronzio - Costa 0 .

Curtis - Curt.

Dalman - Dalm.

De Geer - D. Geer D. Gr. Desvoidy - V. Robinearl.

Dufoul - Durr.

Dumerille - Dumr.

Eabricius - Fabr. Fbr. F.

Fallen - Fall. Fll.

Fargean (De St.) et Serwilie

Genè

$$
\text { - S. F.S. }
$$

Geuffroy - Geofr. Gfr.

Goureall - Gour.

Guerin Meneville - G. Menll.

Haliday - Ilulid. HII.

Holfinansegg - IIof. IIgg.

llliger - Ill.

Laireille - Latr. Itr.

Linneus - Lin $L$.
Lìn - Lw.

Macteay - M. Leay.

Marguart - Macq. Mqt.

Mrinerle - Megrl. Mgl.

Mrigen - Meig. Mgn.

Olivin - Oliv. Ol.

Panzer - Panz. P'.

Passctini - Passer.

P'riris.

I'etagna.

Riobinean Desvoidy $-\boldsymbol{k}$. Desv.

Pondani - Rndn.

Phoser (De) lloser

Rinssi (Falma Eirlisca) Rossi

Rossi (System: Vertz):Rossi

S. V.

Schembri - Scmu.

schmunel - Schm.

Scopoli - Scop. Spl.

Sothiner

skrank - Sk.

Siannius - Sian.

Sliegrer - Stcog.

Stephens - Steph.

Tringui.

Walker - Wlk.

Westwood - Wste.

Wieduan - Wdm.

Winertz - Winz.

Zeller - Zell.

Zetterstedt - Zell. Zt. 
4.

- - 19 basa

- $16-6$ lungrime

- $18-3$ ant

- - 12 Anterix.

- $20-4 a !$

- 45 - 19 ovalus

- 80 - 4 Ancustifrors

- 95 - 12 Uroque

- - - If teli

- $108-11$ Acominatis

- $111-10$ Accuminato

- - 16 Ciliala

- I.3.3 - 13 Noleropliila. quiutam

base

longiores

aut

Antenna

el

ovalus

Angustifrons

Chrogue

tali

acuminato

acuminato

cibiala

Noteromya.

- 14 - 1820 transpone species lypicas IIydroph: licgius et Notalus.

- 196 - 21 Bolithobia. Bolihomya.

- 173 - 6 Chorisosps Mihi: Actina Wull:

- 195 - 5 Lrjomya.

Lejosoma.

- 185 - 6 Bophusia Mihi:

'riciphona Fect: 
it.

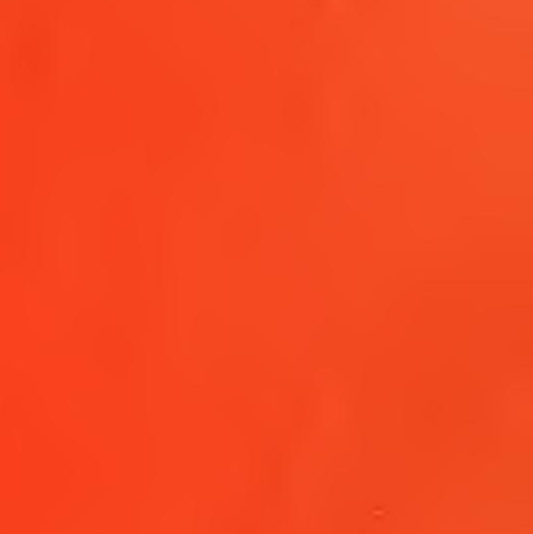





\title{
DIPTEROLOGIA ITALICA
}

\author{
PRODROMUS
}

A. C A

VoL: II. 



\title{
SPECIES ITALICA
}

\section{ORDINIS DIPTERORUM}

IN GENERA CHARACTERIBUS DEFINITA, ORDINATIM COLLECT $£$, METHODO ANALITICA DISTINCTE, ET NOVIS VEL MINUS COGNITIS DESCRIPTIS

\section{AUCTOIR}

\section{CA}

In R. Athleneo Paruenei Professore - Socletatis ENtonologice Parigiensis - R. Accademe Georgofllorum Florentin.a - Societatis Zoologroa ex Botanice WiNdobonensis - AcGadehie Nature curlosorum Neapolitane Societatis Entomologic.s Stetinensis - Rei agrarie Regil Lepidi, et A thanei Bassanensis Sodale etc. etc.

\section{Pars Prima}

OESTRIDE: SVRPFHIDA: CONOPIDA

\author{
P A R M A
}

EX TIPOGRAPHIA ALEXANDRI STOCCHI

$185 \%$. 



\section{MA XI IILIANO SPINOLA}

MARCH :

\section{ENTOMOLOGIE DOCTORUM}

\section{NEMINI SECUNDO}

\section{ITALICORUM PRIMO}

REVERENTI E'T AMICO ANIMO

D. $-\mathrm{A}$ 。 

(स),

$L$

a maggior parle tielle specic che sono qui annoverale furono raccolie nelia provincia parmonse e nelle limitrofe, per cui fu mio mimo pensicro di pubblicare soílanto uno Species dell'Ilatia centrale, ma considerando in seguito che dal lavoro cosi limitato restava eschusa una parte importantissima, colle specie dell' Ausonia meridionale ed alpina che ho potuto radunise nella mia collezione, fra le quali alcune sono nuove per la sciensa o per altri riguardi non poco inreressanti, mi sono determinaro ad estendere $i$ snici studii a lulli $i$ Ditleri della penisola nostra "delle isolg adiacenti, e di pubblicarme questo spucchio sistematico, al quale dowri essere ritcnulo come lavoro non completo, almezio per le specie della bassa Italia, e considerato soltanto come pretudio ad una Dilerologin Iluliana, $e$ come parte dei materiali che si van preparando per la fauna nazionale.

IIo crecluto fosse pregio dell'operr it non aumentane quasi inutilmente il volume colle de. snvizioni delle specie giò conosciute, o in dirvise

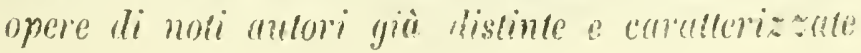


abbastanza, essendo sufficiente di citare, come ho fatto le diagnosi che mi parvero migliori, o piu complete, alle quali si appoggia la distinzione delle specie da me esaminate, aggiungendovi, quando lo credei vantaggioso, ciò di cui mi parevano difettare.

All' oggetto medesimo di non ingrossare senza giovare il lavoro, ho ristretla la sinonimia generica e specifica a quella degli autori principali, e solamente trattandosi di cose italiane non ho trascuvato di citare anche le opere di non molta lena in cui si parla di specie particolar al nostro paese, ed i lavori anche di poca estensione di Entomologi italiani in cui si tratta piu o meno ristretlamente dei Ditteri nostrali.

Per riguardo ai generi non ho potuto escluderne le diagnosi, benchè abbia sempre avuto in vista di abbreviarle quando e per quanto poteva farsi senza nuocere alla precisione, imperocchè alcuni dei medesimi essendo fonslati di nuovo per insetti che prima d'ora non erano conosciuti, ed altri per specie note ma che furono da me disgiunte da generi già adottati; e per molti avendo creduto valere come note distintive generiche, diversi caratteri organici non prima osservati o non creduti importanti, ho veduto necessario di far conoscere le ragioni per le quali questi generi furono da me stabiliti, e separati dagli allvi. 
Nello stesso modo nan ho potuto astenermi dul descrivere le specie che reputo nuove, alfinchè si potessero rilevare $i$ motivi che mi hanno indotto a crederle non descrntte da altri, e quindi autoriszato a distinguerle con nome nuovo.

Tulle le provincic Ilaliane hamno maggiore o minor numero di rappresentanti in questi assemblea di insetii ditteri nazionali, meno quella di Roma, ma essendovi molie specie provenienti da paesi limitropi, piu meridionali e più boreali del romano, si pui ritenere che anche te produsioni dillerolugiche di quella regione interposta vi siano annoverute.

Pochissime specic europese non ancor raccolle da noi avran posto in questo quadro sistematico, e solamente quumlo siano tipj di generi ben distinti, e de muovo salititi, $c$ quando simo yiì conoscinie come abiturici di puesi prossimi ul nostro, e cosi sin probnbile che noll' Italia pisssan essere in seyuito riscombrate.

Fral gli Entomoloni ad Enlonofili Ialiani, (*)

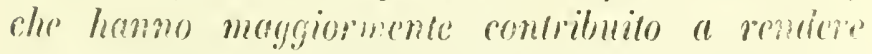
meno incompleta ques:a mie resseyna dei billori Indigeni, mi corre debilo di nominare, Il Pro-

(" I Xora - I dulti seranieri che giovarono all' opera mia cullit spedizime di insmi di diversi pacsi e colle foro osservazioni, somo

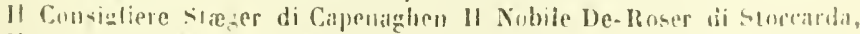

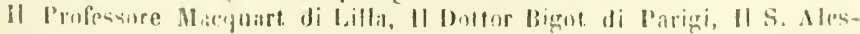

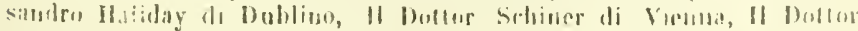

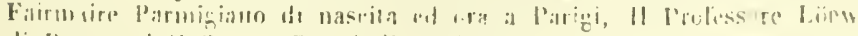

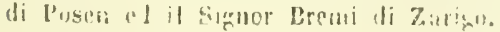


fessore Bellardi di Torino che mi ha feworito molle specio del Piemonte, fra le quali alcune assui importanti dolle alpi: Il Sig. Schembri Ornitologo et entomologo di Malta dal quale ottenni una raccolla ditterologica di quell' Isoba e delle aliacenti: Il Signor Giuscppe Mussino di Gennva il quale mise a mia disposizione una oollezione di inseti Ditteri piemontesi e Liguri: Il Signor Professore Oronsio ed il Dottor Achille Costa di Napoli che mi spedirono diverse volte insetti del regno di quì dal furo e della sicilia: Il fu mio anico e macstro il Cavaliere Professore Genè di Torino che diresse $\mathbf{j}$ mici primi sludii entomologici con altiva ed amorevole corrispondenza e mi communico parechic specie utilissime per me, del piemonte della sardegna e di alli paesi: Il Professore Gemellaro di Catania dal quale otlenni due spedizioni di Insetti siciliani: II Signor. Cavaliere Truqui di Torino che mi speti con altri insetti un invio di Ditteri del Piemonte: I signori fratelli Villa di Milano, per non poche speice lombarde che mi hanno favorito, delle quali alcune assai pregevoli dell' alpi insubriche: Il fu mio corrispondente e collega Conte Carlo Porro di Milano, che mi cedè una sua raccoltina di Inselti Dipteri lombardi, quando si dedicò allo studio eschusivo dei Molluschi: Il Dottor Pecchioli di Disu rhe mi spedi rarie volte le specie dai 
dintorni della sua cilla: Il Conse D'arco di Manlonu pei Dilleri del suo municipio: Il Marchese Hassimiliano Spinola Il grande Entomologo, onore d'Ilalia, il ruicarteggio mi i scuola e sprone a serii studii scienlifici, e dal quale moltissime specie di inselli indigeni ed esolici mi furono regalate, anwhe dell' ordine dei Ditberi, che furono raccolli nella Ligaria, nella Corsicu, nella Sicitia ed in alle provincie; e finalmente $i$ Professori Bertoloni Giuseppe di Bologna, e Passerini Carlo di Firenze, non che il mio dollissimo concilladimo it Doltor Eugenio Berti, i primi per diverse specie, raccolle nei dintorni delle rispettive cilli, l'altro per molti, e non pochi rari inselti dilleri dell' appennino parmense.

Se, come spero, $i$ dotti e zelanti amici della scienza e del paese, vorranno ancora comunicarmi le novilà e specialitì delle diverse provincte di Ausonia per essi abilate non sarib difficile di dare tanta estensione a questo prodromo, da poto in seguito meritare di esscre intitolato

\section{FAUNA DITTEROLOGICA ITAMANA}

Parma 20 Sellembre $185 \%$.

Prof. Ganila lioxinato 
Nomina Stirpium in ina desinentia mutare oportet in ince, ad contusionem vitandam cnm vocabulis genericis pari modo terminantibus, et etiam ut perfecte consona sint cum illis subfamiliarum Systematis Bo- najarlei, yuibus sectionibus Stirpes meæ respondent, el sic

Mo Ostmana Sraphina elc. Lege CEstbina Syrphine elc. Pag. 52 Lin. 12 pro Camprencouna - Campìneuara.

- 55 - 16 - Sighyximoapha - Apiximaguha.

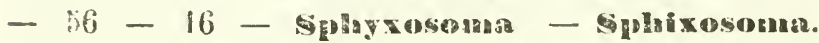

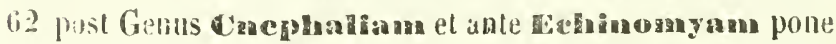

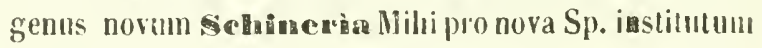
al tergestum lecta el mitai transmissa a Doct:-Schinel Windobonensi.

Char. Generici præcipni sunt. - Aristce articuli duo primi elongati, secundo longiore, el simul longitudinem tertii fere æquantes. - Antenne satis longæ, articulo tertio secundi longitudinem parum superante - Gene non Inmida sed latæ el setulis pluribus sparsis. - Oculi nudi. - Series setarum orales el frontales brevissima - Macrochete discoidales aliyfix: in lorso segmentorum abdoninis intermediortum. - Vimn transversa cxterior alarum satis distang ab innisur, et froxina culito quinta longitulinalis ete. Epee. Fip. S. 'frrastina Mili. 
Pag. 64 Lin. 22 pro Erecbis Desr. - pone erernis Mihi, quia Erebice genus in Lepidopteris jam adoptatum.

- 66 - 14 Tolle Gnava Mgn, et proSpec. Typica pone Quadripustulata Fabr.

- 67 - 11 pro Brevissimæ pone breviores.

- 68 - 19 - Cyrthophideha - Cyurthophawa.

- 75 - Inter gy yrosian el ischerians pone Genus Zelleria Egg.

Distinctum a Prima, segmento secundo abdominis macrochetis destituto, et vena secunda longitudinali ultra transversam interiorem non manifeste elongata; ab æltera proboscide non distincte producta ultra epistomium; ab illa et ista, tarsis anticis longissimis etc.

Sp. Typ. Z. Longimana Egg. - prope Tergestum lecta.

- 87 Lin. 3 pro Ptilocera Mgn. Lege Ptilocheta Mihi.

Ptilocerce Genus a Wiedmannio in stratiominis institutum.

- 88 - 25 - Ante Proboscinan pone genus Ptllops mihi quod continet præcipue species generis Maquartia aristam pilosulam præbentes, et a Proboscinis distinctas, præter alios characteres, proboscide non manifeste producta ultra epistomium, et isto crebre pilosulo, non setis longis marginato.

- 89 Lin. 3 pro Melania Mgn. Lege Melanota Mihi. Melanice nomen in Lepidopteris - Moluscis, et plantis.

- 99 - 4 - Spaziphora - Spathiphora.

- 101 Lin. 25 pro Litorella Rndn. pone Schrenomayza Ulalid.

- 104-19- Heterostoma - Meterochcila quia 
Genus Hetrostoma in Myriapodis jam adest.

- 107 - In Sciomyzinis pone Gemers A ragosion Waga. Cujus Sp. Typica Adap. Codrctata Wagr. prope Terbanum capta teste Schinerio. - Vite Char. Gen. et Spec. in

Annal Soc. Entom Gallix 184?.

- 133 Lin. 13 pro Voteroplaila Lege Noteromag, quia Genus hoc nomine jan distinxit Westwodius.

- 141 - 17 - Anoploneras - Amopingous.

Nomen primum pro genere Coleopterorum 1)ejanius proposuit.

- 143 - 25 - Mammohntes Stann. - Stunẵa Mihi, quia Ammobates genus est Hymenopterorum.

- 175 - 13 - Apogom - Alasion.

a Perrisio genus Apogon jam institutum in Dasipogoninis.

\section{NOMINA AUCTORUM \\ IN PRIMA NOTA NON INSCRIPTA}

$\begin{array}{ll}\text { Angelini - Angl. } & \text { Frauenfeld - Frnf } \\ \text { Egger - Egg. } & \text { Stcnhammar - Stnhu. } \\ \text { Fischer - Fsoh. } & \text { Waga }\end{array}$




\section{FIIILUII.}

\section{ESTRIII}

Colnact: Fanule.

Amemo in caro faciei biloculari inserte; artionlis tribus, præter aristam, præditæ, basali brevissimo, tertio nou circulatim inciso - Arista in dorso articuli tertii sirta - Oris apertura subnulla - Proboscis all palpi non, aut vix distinguendi - Alarum vence longitudinales septem (præter axillarem ${ }^{*}$ ), quinta tantum extrinsecus flexa, vel omnes subrectæ spuriis aliquibus inter primas tres longitudinales plus minasve manifestis - Corpus pilosum sed setis validis non instructum.

(") Asillares sunt, venx spurix qnx oriuntur ab incisione alari prope basim in margine postico, non a radice alarum nec ab aroolis basalibus, sxpissime tota decolores ot exiles. 



\section{Stirps 1. OESTRINE Rndn. (*)}

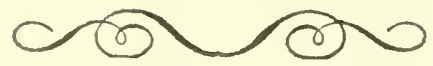

Genus 1 GCSTrRes. Lin: Scop: Fab: Rossi: Latr: Clark: Fall: Macq: Genè: Rndn: $\left({ }^{* *}\right)$

Gastrus Mgn: Zelt.

Gasterophilus Leack: Walk. Curtis.

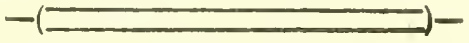

Charagteres Generici.

A.теNאæ brovissimæ, triartieulatæ, articulo ultimo sublenticulari, in earo faciei biloculari lesandæ: Arista dorsuali sabnuda. OcBLL manifesti.

Os clausum, ProBoscide gon manifesta, PALPIS nOD aut vis distioguendis tuberculiformibus.

Caliptera modice ampla, zalterrs non tegentia.

ALB renis longitudinalibus bene observandis septem, partim spuriis ot decoloribue: transversariis tribus quatuorve, quarum daø argolas aNALRS extrinsecus claudentes.

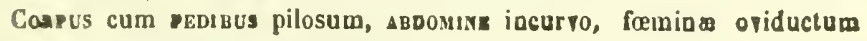
validum sub veutre flexnm.

(") Cenera Italica ordinis Dipteror. $185 \%$.

(“) Annali di Bologna 18ö's. 
Species Italicie.

A. Vence transversaria tres tantum manifestæ.

Spec: 1 Ferruginatus Zell.

AA. Vence transversario quatuol magis vel minus distinctæ. 3. Vence transversarice duæ intermediæ nou aut vix distantes (1).

$$
\text { Spec. } 2 \text { EQuI - Clark. }
$$

BB. Fence duæ transversarie intermediæ satis distantes, infera minus perfecta.

$$
\text { Spec. } 3 \text { Hemorrioidalis - Lin. }
$$

Observationes et Synonimia

Spec. 1 CE. Ferruginatus Zell. Rndn. ) (2)

(" Jubarual V.? Mgn.

« EQvi hossi.

Ville descriptionem Zellerstedlii.

(1) Huic seetioni perliaent species Europer. OE Salutaris Kilr. seconum Fab. et forte alia nondum captie in Italia. OE P'ecorum disinctus erit Thoruce et basi abdominis rulo villosis, Alisque inluunatis, OE. Salutaris difert ab aliis, basi abdominis albido sub trauslucida.

Specierum Nasabis Lin et Clakckn Leach chararteres alarman nou cognoseo, sed ecrte generi isto pertineul, eliamsi cui sectuni ejusdem referendxe sint nesciam - Primæ Speciei Diaguosis, seu OE. Nasalts L. rel Veterisus Fabr. et Fall. Thorate et Abdomen pustice rufo villosi; basi abdominis caua.

Diagnosis OE Charikit Leach. Caput, Thorrex el pedes Sulvo villosi, Abdomen hasi alba pilisque albudis.

(2) V. Opuseulum (Suila pretesa ideutità specifsca degrli Estridi) I’arua 1834 et Anaali di Bologua 1834 . 
Semel lectus prope Parmam, et bis in apennino parmensi: cujus pupx in stercore erquino inventæ.

Pupa post exitum insecti declarati prædita superne cingulis transversis dentatis sic dispositis. - Primum (scilicet quod tangit aperturam anticam) et secundum integra sunt, seu in medio non interrupta: tertium in medio modice, et quartum latius interrupta, quintum ad latera tantum distinctum, sextum vix inferne observandum. A descriptione Rossii, species ab eodem in Etruria lecta Ferruginatce referenda magis quam aliis auctorum nestris.

Si Rossio credimus istius larva in intestino recto equorum vivit.

Spec. 2 (E EQu. Clark. Latr. Meig. Zett. Macq. Bovis Lin. Fab. Rndn. (opusc. c.)

Vide Descripl: Zetterstedtii.

Exemplaria collectionis mex in Italia media et meridionali capta, quorum unum ex insula Melita D. Schembri misit.

Ejus Larvæ in ventriculo Equi vivunt ut primus Vallisnierius observavit.

P'ıpæ post exitum insecti declarati cingulos dentatos exibent sic disposilos. - Primi quatuor, ab apertura antica, non interrupti, quintus in medio interruptus, sextus ad latera tantum el inferne observandus.

Spec. 3 (E Ilemorrhoidals Lin. Fabr. Fall. Meig. Clark. Mac\%. Zett. Wall. Rindn, (Opusc. c.) Vide Descript. Zetterstedtii. 
Tnicum exemplar collectionis meæ a Geneo olim accepi, forte in Pedemontio inventum.

7) hx hujus speciei cinguli dentati, tres primi (ex apertura antica) integri sunt, quartus in medio late interruptiss, anintus inferne tantum observandus, sextus deest. $\left({ }^{*}\right)$

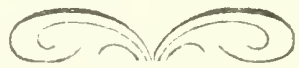

STYRPS II. HYPODERMINÆ Rndn.

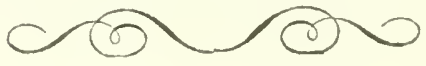

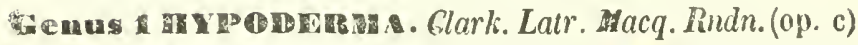
Q2istrus Lin. Fabr. Rossi. Latr. Mgn. Fall. Zett. Walk. Genè.

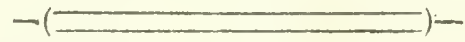

Characteres Generici.

Antenne bevissima, articulo tertio apicali majore subglubuloso, in cavo faciei biloculari locandæ, ABista dorsuali subuuda.

(1) Se avvenga che si scoprano parrecchic snecie almeno esoliche, di questo genere, allora i caratleri alari che sono qui adoperati come specifici potran diventare geverici: e couservando if nome di OEstnux per quel geuere che compreuderù la specie Equi del Clark, agli altri due si adatterebbero i nomi di Gastrus del Mgo. e di Gastenophicus del Leack; ma se è vero come alferma il Rossi che il Ferruginatus vive in istato di larva nell' inlestion retto dei cavalli, i nomı Genorici di Gastrus e Gasternparues conterrebbero un idea falsa e dorrebdero essere cambiali, sostiluendovi quello di Entarantza. Mihi. 
Ocelli distincli.

Caliptera magna halteres tegentia.

Alce renis longitudinalibus distinctis septem, quinta extrat secus cubilata, sursum flexa et sejunctim a quar ${ }^{\circ}$ margini producta, sexta pariter cubitata et qunti: ad apicem conjuncta, cubito appendice venose des!

tuto (*) Areola basilaris seu intermedia unica; Areu-s lis analibus seu interioribns duabus.

Corpus pilosum sed setulis non instructum. Oviductum fominæ porrectum validum.

Species Italica unica 1 Bovis - Clarl.

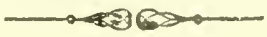

Observationes et Synonimia

Sp. 1. OE Bopis Clark. Falr. Rossi. Latr. Myn. Fi, Macq. Genè. Zett. Rndn. $\left.{ }^{*}\right)$ Walk. Emorriyoldalis Lir.

Vide Descript. Zetterstedtii.

(") Nelle vene appendicolate dei ditteri, alcuni autori consider.m. una tale appendice come la prolungrizione della vena medesima, e 1 . tengono esscre ana vena transversa!e quella porzione di cosa ... parte dill' inserzione dell' appendice e si dirigge rerso la precedte., longitudinale, ma quando l'appendice è spuria o quasi tale o. : brevissima, è più ragionevole di non confonderla colla vera vena s si prolunga piegandesi auche bruscamente.

("i) Getera Ital. Annali di Bologna 1864. 
In agro parmensi exemplaria mea lecta, quorum aliqua $\mathrm{ex}$ larvis domi orla.

Larva vivunt sub cute dorsuali boum.

Pnpæ rugulosæ et verrucosæ sed cingulis dentatis destilulæ. In hoc genera locanda Species Lapponiæ que vocalur $\mathrm{Ta}$ randi a Linneo, et typica Generis CEDemagena Clark.

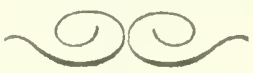

fenus a CEPHARER A Clark. Latr. Macq. Walk. Rndn. (Op. c.).

(Estrus Lin. Fabr. Latr. Fall. Mgn. Genè.

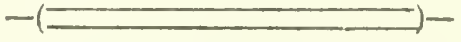

Charact. Generigi.

ANrEKNe articulo apicali subglobuloso, majori, in cavo faciei biloculari locandx; ARIsta dorsusli sed non longe ab apice articuli inserta, nuda, basi distinctæ incrassata.

Os luberculis duobus parvis instructum.

Caliptera magna halteres tegentia.

Ale venis longitudinalibus septem manifestis, quinta et sexta extrinsecus cubitatis, sursum flexis et præcedentibus ad apicem conjunctis.

Corpus parce pilosum el selulis aliquibus prædilam, Frowte, tgonace el Scutello granulosis. 
Species Italiea - Unica. 1 Ovis - Lim.

Observat: et Symonimia

Sp. 1. C. Ovis Lin. Fabr. Latr. Clark. Fail. Rossi. Mgr. Macq. Genè. Zell. Walk. Rndn. (op. c.)

Vide Descript. Zettorstedtii.

Exemplar unicum collectionis meæ domi ortam, ex larva inventa in sinubus frontalibus agni. Mores insecti, Vallisnierius primus observavit et descripsit.

Pupa superne transversim striata, inferne fasciolis decem granulosis cincta.

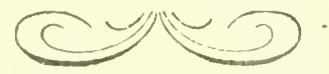

(Nota) Generis CEPUEXEMYA Latr. (OEstros alior) nondum species in Italia Capta.

Forle trium europearum aliqua in regionihus nostris vel alpinis rel apenninis vivere potest, ubi aliæ dipterornm species inventz. fuerunt, in plagis borealibus prius tantum repertæ.

Species melius notæ sic distinguesdx.

A. Corpus pubescens, punctatum, nøn longis pilis hirtum.

1. C. Pacra mihi, Pictug a a

A. Corpus pilis longis hirtum.

B. Alarum basis distinete nigra.

2. Stiwulatrix Fibi

Stmulator Clark. Ngg:

BB. Alarum basis nnn distincte fuscior.

C. Abdomen basi et apiec fulvi hirtum.

3. Aurigarbis miga.

CC. Ablonem nostice tantum lutei rel grisei hirium.

4. Trompe Fulso 


$$
\infty
$$




\section{F A IIILIA I I.}

\section{SYRPHIDE}

Cearact: Familie.

Ansenne articulis tribus instructæ, (stilo vel arista exceptis): articulo tertio haud circulatim inciso. - Gence non inflatæ. - Oris apertura ampla. - Proboscis semper distincta, membranosa, crassa, ultra epistomium non aut parum producta, - Palpi breves, sæpe parum distincti. - Vence longitudinales alarum oclo, præter axillares; quinta et sexta extrinsecus sursum flexis, præcedentes non marginem alarum petentibus. Vena spuria supranumerum plus minusve manifesta inter quartam et quintam longitudinales. - Caliptera parva. - Corpus setis validis destitutum. 
StiRps III. VOLUCELLIN R Rndn. (Gen. Ital:)

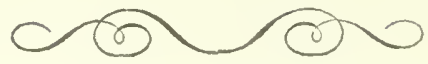

Genus 1. SERECGE Mign. Latr. Macq. Zett. Rndr. (1) Walk.

sxnenuc. Fabr. Fall.

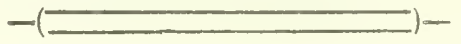

Cuaract. Generigi.

Axrexne contra faciem flexæ, articulo ultimo sublenticulari vel subquadrato, ialsra tota pilosa, plumata ad basim articuli sita. Ocun nudi, in frotste maris superne contigui. Penistomion inferne satis productum.

Ale venis longitudinalibus secnuda et tertia sejunetim costalem attingentibus.

SPECIES ItaLick.

A. Pedes nigricantes. - Abdomen nigrum, basi et apice albidi pilosum.

Sp. 4. Bonibirormis Fall,

(1) Annali di Bologna 1844. 
AA. Pedes rufescentes. - Aldomen nigrum, treniss tribus transversis flavido albidis.

\author{
Sp. 2. Lappona Lin.
}

Osservat. et Synon.

Sp. 1. Bombiforairs Fall. Mgn. Macq. Zett. Rndn.

v. Descript Zetterstedtii.

Exemplaria collectionis meæ in Alpibus lecta, quorum duo in Monte Rosa capta Prof. Bellardi taurinensis misit.

Sp. 2. S. Lappona Lin. Meig. Macq. Zelt. Walk. Lapponux Fabr. Fall.

v. Descript Zetterstedtii.

In alpibus pedemontanis a Prof. Bellardi non raro lecta.

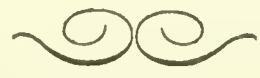

(Nota) Species Generis hujus Borealis, Fallegil et Mussiravs Meigen facilius in Alpibus italicis vel apenniuis elatiorbus inve. niendæ.

V. descript. in oper. Meigenii Macquartii et Zetlerstedthi. 
Cenue voldceld Geofr. Latr. Mgn. Macq. Curtis Zett. Rndn. Walk.

- rareus Fabr. Fall. Panz.

conere Scop.

wueca Lin. Degeer. Fabr. Rossi. Patagna.

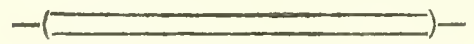

Charag: Gener:

ANTENme arliculo tertio distincte elongato, ARIsta ad basim articuli inserta et lota plumala.

Ocub saltem in mare hirti, in quo seru vet in fronte superno subcontigui, vel satis approximati.

A se venis longitudinalibus secunda et tertia conjunctis ante costalem. Peassтomium eatis inferne productum.

\section{SPecies Italica}

A. Abdomen flavo fulvescens tæniis transversis nigris.

B. Abdomen superne segmentis tribus nigromarginatis, ventre basi tota flavicante.

Sp. 1. Ixanis Lin.

BB. Abdomen superne segmentis duobus Ianlum nigromarginatis, vontre basi late nigra.

Sp. 2. Bifasciata Scop. 
AA. Abdomers niģrum, vel basi lutea aut albida, translucida, vel rufi, lutei ant albidi longe pilosum, saltem apice.

C. Abdomen basi lutei vel albidi, translucida.

D. Thorax dorso flavido piloso - Tarsi basi rufescerte picea. Sp. 3. Dryaphila Scop.

DD. Thorax dorso breviter nigro piloso - Tarsi toti nigri. $S p$. 4. Prluucens Lin.

C.́. Abdomen nigrum basi non distincte translucida, sed albici, Fulvi, vel lutei longe pilosum, sa!tem apice.

E. Thorax et scutellum omnino nigro hirta.

Sp. 5. Bourbylans Lin.

EE. Thorax vel disco nigro, lateribus et scutello flavido pilosis, vel omnino etiam disco pilis flavicantibus hirtus ut scutellum.

F. Thorax disco uigro, lateribus ut soutellum flavido pilosis. G. Antenna articulo tertio flavido - Pili anales pallide lutei. Sp. 6. Mrstacen Lin.

GG. Antennæ tolæ nigricantes - Pili anales rufo-ferruginei.

Sp. 7 Alpicola Milıi.

EF. Thorax totus etiam disco ut scutellum flavo-pilosus.

Sp. 8. Proxıma Mihi 
30

Observat. et Synonim.

Sp. 1 V. Inans Lin. Rossi. Mgn. Fall. Latr. Macq. Zclt. Rndn. Walk.

Migans. Fabr. Fall.

v. Descript. Meigenii et Macquartii.

In dilione Parmensi frequens tam in planitie quam in montuosis, a mense majo ad mensem augusti; præsertim legitur in floribus arbustorum et sepibus.

in pedemontio quoque non raro legit Bellardi.

Sp. 2 V. Birasilata Seop.

Zomaria Mgn. Macq.

Imanis Fabr. P'elagna

v. Descript. Meigenii et Macquartii.

Vulgaris in toto agro parmensi, præsertin ab aprile ad septembrem.

E Pedemontio hijus speciei exemplaria misil Bellardi, a Liguria Mussino, a Bononia Bertoloni, a Sicilia Gemeilaro, a Mantua D' Arco, a Mediolano Villa etc.

Sp. 5 V. Dayapulla Scop. Inflata Fubr. Mgn. Macq. Walk. Curlis. Pellugens Rossi.

v. Descript. Meigenii et Macquartii.

Parmæ nou rara mensibus aprile majo el junio, in floribus præsertim arbustortur. 
in pedemontio legit Bellardi, In Liguria Mussino speciem invenit, et ad Tergestum D. Schiner.

Sp. 4 V. Pellucens Lin. Fabr. Latr. Fall. Mgn. Macq. Walk. V. Descrip. Meigenii et Macquartii

Non frequens in collibus et apennino parmensibus primo vere.

In Pedemontio Bellardi, in Sicilia Costa legerunt et mihi speciei exemplaria miserunt.

Sp. 3 V. Bonbylans Lin. Fabr. Fall. Latr. Mgn. Macq. Walk.

v. Descript. Meigenii et Macquartii.

Rara in coliilus et apennino parmensibus.

In locis alpinis Pedemontii legit speciem D. Bellardi.

Variat abdomine pilis analihus albicantibus et lutescentibus, Antennis aticulo tertio paulo luteo, Scutello fusco lutescente nou niģricante elc.

Sp. 6 V. Mystacea Lin. Fabr. Lalr.

Pequata Scop.

Plunaza Fall. Mlgn. Mlacq. Zett.

V. Descript. Meigenii et Nacquartii.

Infrequens in ditionis parmensis collibus et nontibus. In Alpibus lnsubrixe species lecta a Clar: De Cristofori, in Pedemontio, a Truqui et Bellardi.

Variat abdonine pilis apricalibus albidis luteis et ruis. 


\section{Sp. 7 V. Alpicola Mihi.}

Similis abitu el statura V. Mistaceæ, exceptis antennis in hac totis nigricantibus vel piceis, et pilis analibus rufo ferrugineis non lutescentibus.

as collectionis mex difert etiam lateribus abdominis totis rufohirtis non in medio nigropilosis, et etiam aristae stipite nigric ante.

Fomince vero abdomen et thorax minus late nigro pilosa. Masculum ab Equite Truqui Taurinensi accepi, Fœminam fratres Villa in Alpibus Insubriæ captam miserunt.

\section{Sp. 8 V. Proxira Mihi.}

Similis Mistacece at distincta, thorace superne ommino fulvo hirto, vix pilis aliquibus raris nigris sparsis, non antice macula magna quadrata nigro pilosa prædito. Bis capta in Apennino parmensi, semel a Doctore Bertèo, et a me semel.

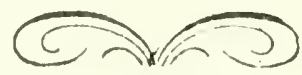




\section{Stirps IV. ERISTALIN E. Rndn.}

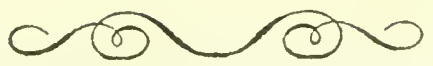

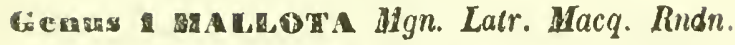

Womstazan Fabr. Latr.

Sxnepuc. Fall.

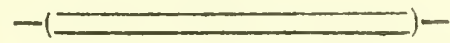

Charact. Gener.

ntennce articulo tertio longitudine latiore, arista buda, dorsuali, in medio circiter articuli inserta.

Oculi in atroque sexu hirti e in fronte distantes $(\%$

Pedes fernoribus non infatis sed validi; apophisi nalla ad apicem fo. morum posticorum nee promiuentia iofera instructis: tibiis a

(1) Species extra Italiam leetæe oculis nudis, generi distincto adscribendæ quod appellabitur - Ledærs mihi. Cujus Spec. Typ. Mallota Vittata Mga. nisi G. Zerrenstemese pertinent vel HEROPEILIS.

Generis Lejopsimes Claracteres distinctivi si adoptati; Antennoc articulo tertio latitndine latiori, arista sub-apicali nuda - Oculi nudi, in utroque sexu superne sejuncti - Pedes femoribus posticis dilatato compressis, tibiis vis et totis regulariter latiusculis; femorum Inngitudine satis hrevioribus etc.

Ale circiter ut in Gen. Sequeate. 
medio ad apicem dilatatis femorum longitudiue parum brevio= ribus.

Ala renis longitadinalibus secunda et tertia sejunctim costalem attingeatibas.

Species Italiga Unica 1. Fuelfornis - Fabr.

Obscreat. el Synon.

sp. 1 M. Fugiforuis Fabr. Lair. Mgn. Macq. Rndn.

v. Descript. Meigenii et Macquartii.

Non rara in floribus arbustorum primo vere tam in planitie quam in collibus ditionis parmensis; inventa etiam in Pedemontio a Bellardi.

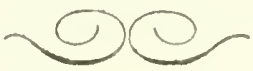

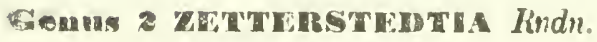

Srempars Fall. Zett.

Hinistakis Mgn.

Marmota Löeto.

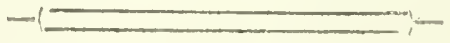




\section{Charact. Genek.}

Antenne articulo tertio longitudine latiore, arista dorsuali ouda in medio articuli tertii inserta.

Oculi in utroque sexu nudi, in fronte maris superne anguste contigui. p.d.s femoribus prasertim posticis satis inflato-incrassatis, apophisi subapicali nulla sed prominentia infera præditis, tibis totis dilalatis, femoribus sub æque longis.

Alo veris longifudinalibus secuuda et tertia sejunctim costalem attingentibus (1).

Species Italiga Unica 1 Cymbiciformes fiall.

Observat. et Synon.

Sp. 1 Z. Cymbtciforuis. Fail. Meig. Zett. Rndn.

V. Descript Zetterstedtii.

(1) Dominus Loëwius in epusculo quarto Diplerulogico (Berolini 1856 ) genus hoc inutilem esse dieit, quia species typica ejusdea cerle Mallotis pertinet. Hæc sententia facilius adoptanda si omnes Malloto Meigenii, oculos nudos, in fronte maris subcontiguos et femora in ulroque sexu inflata proberent ut. Sp. Cymbiciformis Fallenii; sed rejicienda, quia species continet oculis hirtis, in fronte ulrinsque sexus distantibus, et femoribus, non distincte incrassatis, us in Furiforme Meigenii observatur. 
Parmæ non frequens mensibus majo et junio - In flotibus variis pratensibus et arbustorum, in locis paludosis flores Euphorbix palustris frequentat (1).

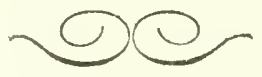

Gemus 3. Phatrivacheres Wiedm. Macq. Rndn. Winestakes Fabr.

Cuaragt. Generigl.

Antcunce articulo tertio ovato subacuminato, secundo longitudine tertii, cylindrico, ad ipicen crassiore; primo brevissimo: ariste dorsuali, nuda, in medietate basali articuli tertii sita, maris ad apicem distiocte dilatata.

Nemo certe nunc conjungat generice species istas, characteribus tanti fratii diversas; ideo Mal. Fuciformem typieam geueris servaudo, alias distincto generi referendas esse, anno $184 \%$ defendebam.

Nou credo Doun. Loëwium nec alium nunc species tam distinctas conjunctas servare posse.

(1) Speciem hoc uomiue spellatan a Meigeoio Zetterstedtio et a meipso, Dom. Loëw, non credu ayua!em Cymbiciformi Fallenii, et in opusculo s. c. eam nuncupat Eristaloidem, afirmando in collectione propria extare specimina quæ certe referendil sunt diptero FalJeniano . . .

Si hoc verum, nomen inmulandum, sed argumenta uon refert, quibus appareat, suam, usn illam anctorum, Cymbiriformi, Fallenii gertinere .. Quia non descripsil indirioua diversa collectinnis suar 
Oculi nudi, in tronte maris angustissime subcontigui.

Ala ciriter ut in generibus precedentibus venosa sed in curvatura venæ quintæ longitudiualis appendiculæ duæ adsuot, una interior altera exterior.

Pedes robusti sed femora non distinete infata nez apophisi valida apicali prædita.

Species Italica unica 1. Serosus Fabr.

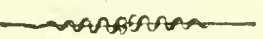

Observat: et Synonimia

Sp. 1 P. Setosus Fabr. Wiedm. Macq, Rndn.

V. Descrip. Macquartii

In Ilalia magis meridionali vivit, ex insula melita exemplaria duo speciei a D. Schembri accepi, unum $e$ Sicilia a D. Costa.

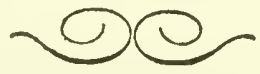

sod nostræ characteres ennumeravit, jam et optime a Zetterstedtio descripla.

Nisi rationes cognoscantur quibus necessaria sit mutatio. $S p$. nostram Cymbiciformem vocabimus, cum Meigenio et Zotterstediso, Pallenii nemen servando. 
Genng 4. Fimstads Fabr. Latr. Mgn. Macq. Loëio. Rndn.

Cormermus Latr. Sxnpnue Fabr. Rossi. Panz. Fall. Zett.

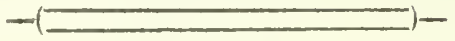

Chanact. Generici.

Antennce articulo tertio subcirculari, secundo breviose, primo brevissimo: arista dorsuali, plumata, pilosa, vel pubescen'e.

Oculi hirti, in fronte maris vel antice subcontigui, vel distincte approximati.

Alce venis longitudinalibus secunda et tertia at apicem conjunctis ante costalem, appendiculis nullis in curvatura quartæ.

Pedes validi sed femoribus non distincte intatis, nec apohisi valida apicali præditis.

(Nota) Si placet Eristalorum species facilius in genera tria sic distincta distribuere possumus.

A. Arista plumata vel distincte pilosa

Gen. 1. ERistalis Fabr.

Spec. Typ. E. Siмııis Fall.

AA. Arista breviter pubescens vel tomentosa tantum. B. Oculi maris in fronte coherentes vel subcontigui.

Gen. 2. Eristalomya Mibi.

Spec. Typ. E. Tenax Lin.

BB. Oculi maris in fronte manifesto distantes, nnn subcontigui.

Geo. 3 Emistaunus Mihi.

Spec. Typ. E. Septrcralis lin. 
SPECIES Italica.

* Subdivisio I. ERISTalis. Fabr.

A. Tarsi postici toti nigri vel nigricantes.

B. Tarsi quatuor anteriores luteo albicantes.

Sp. 1 Sinilus Fall.

BB. Tarsi ymatuor anteriores nigri, basi tantum intermedio rum et aliquando etiam anticorum lutescente.

C. Frons retro antennas pilis uigricantibus hirta.

Sp. 3. Pascuoruar Radn.

CC. Frons retro antennas luteo pilosa.

D. Facies in medio vitta perpendiculari nigra magis rel minus lata et semper distincta.

E. Abdomen segmentis nullis postice luteo-albidimarginatis. Sp. 5. Intricarius Lin.

EE. Abdomen segmentis tribus vel duobus postice luteom albidi marginatis.

F. Frons pilis lutescentibus fascia transversa pilorum nigricantium. - Ala sub-immaculatæ.

\section{Sp. 4. Nemorum Lin.}

FF. Frons pilis omnibus lutescentibus - Ala macula satis distincta nigricante al medium circiter marginis anterioris.

$$
\text { Sp. 3. Orticola De-geer. }
$$

PI). Facies in medio haud nigro vittata, vel linea tantum parum distincia et satis exili nigricante.

Sp. C. Arbestorum Fabr. 
AA. Tarsi postici magis vel minus late albido-lutescentes. G. Frons pilis prope antennas lutescentibus.

Sp. 7. Ruplux Mgn.

GG. Frons pilis prope antennas nigris.

Sp. 8. Saltudat Mihi.

**. Subdiv. II. ERISTALOMYA Mihi.

A. Abdomen nigricante-piceum, sæpits rufo-naculatum vel fasciatum.

B. Tarsi postici toti nigri - Oculi unicolores.

Sp. 9. Tenax Lin.

BB. Tarsi postici late albido lutescentes - Oculi fasciati. Sp. 10. Pulcricers Mgn.

AA. Abdomen nigro œnescens unicolor Sp. 11. CEneus Scop.

***. Subdiv. Ill. ERISTALINUS Mihi.

Sp. Unica

Sp. 12. Sepulcralis Lin.

Mangeroran

Observationes et Synonimia

Sp. 1 E. Sumeis Fall. Mgn. Macq. Loëro. Zett. Walk. Vide Descript. Zetterstedtii.

Frequens in ditione parmensi etiam apenninica, a mense martio usque ad octobrem - Varial abdomine mil- 
culis el lineis minns vel magis latis et manifestis, sed tars: antici et intermedii in nostratibus semper albicantes.

\section{Sp. 2. Pascuorum Rndn.}

\section{Pratoruar Mgrl. Mgn.}

V. Descript. Meigenii.

(Nota) Nomen immutavi quia species altera Pratorum in exotiois eristalibus adest.

Frequens temporibus verno et autumuali in agro parmensi, sed in boreali et meridionali Italia eliam vivit, nam exemplaria aliqua speciei ex insula Melita Schembri, el ex alptbus pedemoutanis Prof. Bellardi miserunt.

Variat abdominis macnlis ut in pracedente magis vel minus lalis et perspicuis.

Sp. 3. E. Ixtricanius Lin. Fabr. Rossi. Fall. Mgn. Macq. Zelt.

\section{Aureus Panz.}

Bombylforomis Fabr. Panz.

Vide Descript: Zetterstedtii.

Vivil in Etruria, teste Rossio. Exemplaria collectionis mere germanica non italica, a D. $^{\circ}$ De-Roser studgartiense missa.

Sp 4. E. Nemorum Lin. Fabr. Rossi. Latr. Mgn. Mucq. Zctl. Walk.

Pertinax Scop. (non Rossi). 
V. Descript Zetterstedtii.

Frequens in planitie et montuosis agri parmensis a marlio ad octobrem.

In pedemontio usque ad alpes a Bellardi el Truqui captus, in regno neapolitano Doct. Costa speciem legit. et is Etruria Rossius.

Sp. 5. E. Horticola De Geer. Mgn: Macq. Loëw. Zelt. Walk. Flayicinctis Fabr: Fall: Zett. Vide Descript. Zellerstedlii.

In Italia pedemontana raro captus a Bellardi a qno exemplaria speciei accepi. Sed ad Tergestum quoque inveniendus teste Schinero.

Sp. 6. E. Arbustorun Lin. Fabr. Rossi. Latr. Mgn. Macq. Loëw. Zetı. Walk.

V. Descript. Zetterstedtii.

Communissimus in tota Italia ab Alpibus at Siciliam; in ditione parmensi a februario usque ad novembrem legilus.

Sp. 7. E. Rupium Fabr. Mgn. Maeq. Walk.

Piceus Fall. Zelt.

Criptarca lanz.

V. Hescriph. Zellerstedtit. 
In Italia Alpina et subalpina speciem legerunt Bellardi Truqui et Mussino.

Sp. 8. E. Saltudur Mihi.

Similis Erist. Rupium, at satis distinctus, pilis capitis tolis nigris et cpistomio lateribus hatd albido-pollinosis. Marem bis obtimui ex alpibus pedemontanis, una vice a $\mathrm{D}$. Truqui altera a Pruf. Bellardi. Fœminam a Tiguro I). Brensi misit.

Sp. 9. E, Tevax Lin. Fabr. Rossi Eatr. Fall. Mgn. Macq. Zett. Loëw. Rodn. Walk.

Vulgaris Scop.

V. Description. Zetterstediti.

Vulgatissimus in tota Italia ab alpibus pedemontanis ad insulam melitam.

In ditione parnensi etiam in tempore hiemali aliquando legitır.

Variat maxime maculis abdominalibus; ita ut species distin-o ctas e varietatibus nonnulli instituerent.

Sp. 10. E Pulcricers Mgn. Macq.

v. Descript. Meigenii et Macquartii.

In Italia meridionali vivit: ex Regno neapolitano Costa, c Sicilia Gemell «ro, a melita Schembri exemplaria aliqua speciei communicála. 
Sp. 11. E. (Gineus Scop. Kabr. Kossi Ngn.

v. Descript. Meigeaii et Macquartii.

In tota Italia vivit ab alpibus pedemontanis ad insulam Melitam. In agro parmensi frequens fere nbiquie et in toto auno, tempore hiemali magis frigido excepto.

Sp. 19. E. Stpulcralis Lin. Fabr. Latr. Macq. Loëw. Kindn. Wulk.

Tristis Fabr. Fall. Panz.

v. Deseript. Macquartii.

In Italia tota et insulis adjacentibus vivit, tamen in insula melita D. Schembri non legit speciem, in Sicilia vero Gemellaro invenit. - Parmæ vnlgaris præsertim mensibus aprile majo et junio.

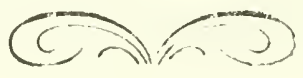

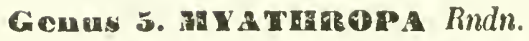

Ezagtane Fabr. Mgn. Macq. Loëw. Walk. Costa. Erepenes Latr.

Srnpnis Fall. Zett. Rossi.

Misea Lin. DeGeer.

Corors. Scop.

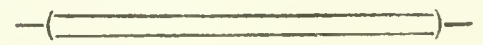


Charactzanes Gerenicl.

cimile characteribus Cappitis Pedun etc generi precedeati, sed satis distinetam præsertim venis alurum secunda et tertia sejunclims costalem altingentibus. -

1 novum a Bhusca athropos Schr.

Spreies Itralica unica 1. Florea Lili.

Observat. et Synomin

Sp. I. M. Flones Lin. DeGeer.

Floreus Mgn. Fabr. Mossi Macq. Zell. Rniln. Walk.

V. Descript. Meigenii et Macqnattii.

In tota ltalia vulgatissima usque ad Melitam, a locis alpinis et apenninis. - In Agro parmensi legitur fere in toto anno.

Variat plurinie colore fulvo luteo et nigricante pelum et fasciarum abdominis.

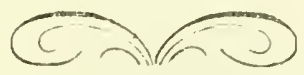


sicnus B. DEDE Macq. Rndn.

Evic: Mgn.

Sxtruxs Fabr. Mgn. Panz. Walk.

Sicriva Fall. Zetl.

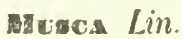

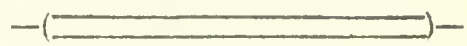

Characteres Generici.

Antennce articulo tertio sub-ovato elongato: arista dorsuali nuda.

Facies paulo gibbosa.

Oculi nudi vel subnudi seu parce et brevissime puberuli, in fronte maris contigui.

Pedes graciles, apophisi nulla ad apicem femorum.

Abdomen depressum latiusculum.

Ala vena quarta longitudinali minus incurvata quam in generibus stirpis hujus jam descriptis, secunda et tertia longitudinalib:is sejunctim costalem attingentibus.

Species Italici.

IValteres capilulo flavo albido.

sp. 1. Fascinta Macq.

(Nota) In methodo uaturali, genus ho in Sykphisis meli:: dquan in Enstabis locum habebit. 
A.. Halteres capitulo nigricante.

Sp. 2 ALx: Fall.

Observ. el Synonim.

Sp. 1. D. Fasciata Macq. Rndn.

v. Descript. Macquartii.

Non rara in agri Parmensis planitie et collibus a mense aprile usque ad ociobrem.

sp. 2. Alneti Fall. Mgn. Macq. Zelt. Walk. Guaucius Panz.

v. Descript. Zellerstediii.

In Pedemontio raro legit. Prof. Bellardi.

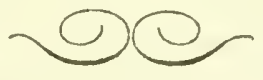

Senus ซ. Menopianes Mgn. Latr. Maeq. Zott. Lö̈w. Rndn. Walk.

Esmistaces Fabr. Minerucs Fall. Rossi

Emveia Fabr.

Nilrea $\operatorname{Lin}$.

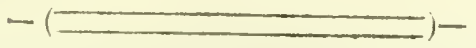




\section{Cinarac: Gener:}

Antennoe articulo tertio circulari, arista dorsuali nuda.

Oculi nudi, etiam in fronte maris remoti vel vix et angustissime antice subcontigui.

Pedes. femoribus posticis satis incrassatis sed apophisi apicali non praditis; tibiis posticis arenatis denticulo terminante, vel paulo intos acuminatis.

Spec. 1talice.

A. Epistomium elongatum subrostriforme - Antenno articulo tertio rufo.

$$
S p \text { 1. Lineatos Fabr. }
$$

AA. Epistomium gibbosum sed non subrostriforme. - Antenna articulo tertio nigro.

B. Tarsi antici distincte dilatati, articulo 2 et sæquente longitudine latioribus: Oculi maris angustissime antice sub-contigui.

$$
\text { Sp. 9. Peregrinus Loèw. }
$$

BB. Tarsi nnn salis dilatati, articulo 2. non longitudine latiore - Oevli in utroque sexu distantes.

C. Tarsi an:ici et intermedii toti lutescentes.

Sp. 3. Trivitratus Fahr.

CC. Tarsi antici toti, internedii saltem apice nigri. 
D. Ablomen fasciis terlii et quarti segmenti in medio distincte interruptis.

$$
\text { Sp. } 4 \text { Pendulus Lin. }
$$

D1). Abdomen fasciis terti et quarti segmenti in medio excaratis, sed non interruptis.

$$
\text { Sp. } 5 \text { Solitarius Mihi. }
$$

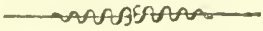

Observat. et Synon.

Sp. 1. H. Lineates Fabr. Fall. BIgn. Macq. Zett. Loëro. Walk.

Muscarius Fabr. Panz.

V. Description. Zettersted tii.

Semel ex pedemontio accepi a Prof. Genèo exemplar speciei. In regno neapolitano quoque vivit teste Doct. Costa.

Sp. 2. II. Peregrinus Loëw.

Oculi maris antice angustissime subcontigui, et tarsi antici distincte dilatati facile speciem ab affinibus sejungunt quibus adle $F$ acies non vittata et $\boldsymbol{F}_{\boldsymbol{C}}$ mora fere toia nigra. Exemplar utriusque sexus possideo a Clar. Schinerio missa, ut refert in Sicilia capta. Fomina tarsos anteriores ut tibioe nigriores possidet. (1)

(1) Pro bac Sprecic, ab affinibus sejuncta, Genus novum institui propouro, characteribus bisce distinctun. Tarsi antici in atroque sexu 
Sp. 5. H. Taivitataves Fabr. Agr. Macq. Loèzo. Zelt. Walk. Perdelus Rossi.

V. Descript. Zetlerstedtii.

Non frequens in agro parmensi ab aprile an octobrem; ah Insubria exemplar sueciri obtinui a D. De-Cristofori.

Sp. 4. H. Pewdulus Lin. Fabr. Latr. Mgn. Macq. Zett. Loc̈ Rndn. Walk.

Precox Russi.

V. Descript. Zelterstedtii.

Frequens in ditionis parmensis planitie el collibus a mense Junio ad Septembrem - Ex pedemontio exemplaria accepi speciei a Bellardi et Truqui, ex Liguria a llussino, et ab Insubria a Conite D'Arco.

Sp. ‡. HI. Solitamus Mihi.

Semel fomina inventa in collibus subapenninis ditionis par mensis et semel in planilie.

Distincta a Pendulo, vel a Triviltato, Abdomine fasciis segmentorum tertii quarti et quinti non interruplis, quamris postice in medio emarginalis - Fuscia se-

dilatati, articulis secundo et sæquentibus longitudine latioribus, Oculi nodi, maris in froute antice subcontigui angustisstnı. Cœiera ut in Holophilis:

Genus apellandum lolle a characteribus 
cunli segmenti lulescente extrinsecus, vel rufescente, $\mathrm{et}$ in medio albidi pollinosa, ut totæ sequentes - Scgmentis onnnibus non, vel angustissime luteo-marginalis pestice. - Facie haud nigro-viltata - Pedibus posticis, femoribus summo apice tantum, et tibiis vix all hasim, anguste flavescentibus.

Specinen collinum fuscias angustiores abdominis prabet.

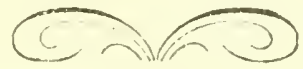

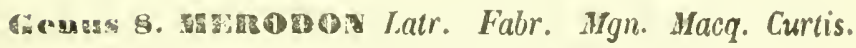
Rndn. (1) Walk.

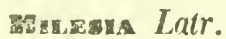

Avapes Fabr. Rossi. Panz.

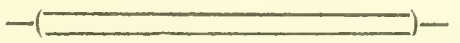

Charact. Generici.

Antenne articulo tertio ovato, vel ovato-elongato, vel raro longo subprysmatico, et rarissimo breviter ovato seu subeirculari: Arista dorsuali nuda, articulis duobus sepius distinctis, primo basali brevi crassiusculo.

I) Annali di Bulogna 189 (sulle Sp. Ital, del Gen. Merodor). 
Oculi pilosi, in fronte maris magis vel minus late coutiguí vel subcontigui.

Facies nec carinata nec tuberculata.

Pedes validi, femuribus posticis satis incrassatis et rpuphisi valida subapicali inferne praditis.

Ale ut in Myatropa circiter venocs.

Species Italice.

A. Coxœ posticæ apophisi longiuscula vel satis longa prasdile saltem im mare.

B. Thorax non omnino lutei vel fulvi pilosus, sed fascia transversa distincta pilorum nigrorum.

C. Abdomen maris segmento qua!to pilis fulvescentibus, vel luteis piloso.

\section{Sp. 1 Pudicus Bellardi.}

CC. Abdomen maris segmento quarto fascia transversa sat lata pilorum nigrorum.

D. Abdomen maris semmento tertin, retro observato, villis duabus transversis distinguendis albido-pollinosis, fascia pilorum nigrorum prope anali angsusta el non valile manifesta. Mncro coxarum conicus.

$$
\text { Sp. } 2 \text { Sunfasciatus Rndn. }
$$

nD. Abdomen maris segmenio tertio vittis albicautibus nullis: fascia prope auali pilorum nigrorum !ata el distinclissima. Mucro coxarum cochleariformis.

Sp. T. Cinerees Fabr. 
biB. Thorax pilis fulvis rel luteis ommino tectus, fuscia nulla transversa nigricante.

F. Tivio posticie maris ad apicen simplices, seu nec tuberculata nec mucronatæ elc.

x. Oculi pilis nigrricantibus superne hirti - Antemne art. $\vec{\jmath}$ nigricante.

$$
\text { Sp. 4. Aneus Mgrl. }
$$

XX. Oculi pilis omnino albicantibus hirti. Antennee art. 3 rufescente.

\section{Sp. $\$$ Erarius Mihi.}

EE. Tibice postice Inaris apophisi aliqua instructæ vel apicali vel subapicali.

F. Tibice posticæ maris apophisi parva acuminata apicali, et tuberculo interiori subapicali præditæ.

$$
\text { Sp. } 6 \text { Mucronatus Mihi. }
$$

FF Tibia postice maris appendicibus apicalibus satis validis instructæ.

$$
\text { Sp. 7. Armipes Rndn. }
$$

Aג. Coxœ posticæ mnticæe, vel vix aliquando tuberculo brevissimo instructe in utroque sexu.

G Tibice postice maris intus tuberculatæ.

H. Thorax antice pilis rufis, postice uigris hirtus - Abdomen basi nigro, apice fulvo vel luteo-pilosum.

Sp. 8 Bulbonus Rndn.

HiH. Corpus totum rufo vel fulvo-pilosum.

Sp. 9. Tubergulatus Rndn. 
GG. Tibice posticx in utroque sexusinnlices, sen non whrelculatx.

I. Tibiæ tolæ nigræ, vel aliquando in geniculis tantum, vel prope geniculos angustissime rufescenles.

K. Thorax villis albidis non manifestis.

L. Halteres toti albido-lutescentes.

$$
\text { Sp. 10. Clavipes Fabr. }
$$

LL. Halteres capitalo nigricante.

Sp. 11. SenILIS M1\%n.

KK. Thorax villis quatuor albidis magis vel mimas distinctis.

$$
\text { Sp. 12. Italicus Rindn. }
$$

11. Tibiæ totæ, late, aut non anguste rufiscentes ad basims. M. Tarsi antici nigri rel nigricantes superne vel ad ayicem. N. Frons ut vortex omnino pilis fulvis hirla. Sp. 15, Montanus Mihi.

NN. Pili verticis antice nigricantes postice fulvi.

O. Abdomen nigro-anescens unicolor, non rufomaculatum.

S. Tarsi saltem superne toli nigricanles.

$$
\text { Sp. 14. Submetallicus Mibi. }
$$

SS. Tarsi ad basim plus minusve rufescentes.

$$
\text { Sp. 15. Funestus labr. }
$$

00. Abdomen magis vel minus late rufo-maculatum.

P. Abdomen fasciolis albicantibus transrersis subparallelis margini segmentorum. Thorax viltis albicantibus tribus intermediis, el duabus lateralibus, sæpe papum perspicuis.

Sp. 16. Vaaus Muln. 
Pl. Aulomen fascinlis albidis oblinuis, non parallelis incisuris segmentorum. Thorax vittis duabus albilis intermerliis salis perspicuis, (non tribus) prater duas laterales.

\section{Sp. 17. Nigritarsis Rndn.}

Mi1. Tarsi antici fulrescentes etiam apice.

Q. Tibrie posticie in medio magis vel miumslate nigricantes. R. Antenne articulo tertio sub-circulari.

$$
\text { Sip. 18. Sicanus Rndu. }
$$

RR. Antennce arliculo tertio oblongo-ovato.

Sp. 19. Spinipes Fabr.

QQ. Tibia posticæ omnino rufescentes.

Sp. 20. Rufitibius. Rndn.

(Nota ) Nel quadro sistematico qui riportato, le specie sono in grao parte distiute per caralteri organici dei soli maschi, cuure neli: mia nota del 1843. II volume era già pronto per la stampa quandis fui pregato da un mio amico di escludere questi caratteri, onde st possano determinare anche le specie di cui si conosce la sold temin: per la qual cosa bo creduto beue di aggiungere al primo, uu altru specchio analitico un quale la distinzione delle specie è basata sovia unte caraterisilche comuni a due sessi, cosi come segue.

A. Pedes omnino nigri, etiam ad basim tibiarum; vix alıg̨eando geniculis anguatissime rufescentibus. 
56

Observationes et Synonimia

Sp. 1. M. Pubicus Bell. Sp. n. (in Litteris).

Similis Mer. Cinereo Fabr. sed satis distinctus Segmento quarto abdominis pilis aureo-fulvis tecto, et non nigris. Species raro lecla in Pedemontio a Prof. Bellardi a gquo exemplar masculum obtinui.

B. Antennœ articulo tertio saltem quadrilougioro præcedente. Thorax dorso grisei-lineato.

Sp. 12. Italicus Rndn.

BB. Antenne articulo tertio ad summum trilongiore secundo. Thorax haud grisei-linealus.

C. Thorax partim uigro-pilosus.

D. Scutellum grisei-pilosum.

E. Halteres capitulo nigricante.

Sp. 11. Senilus Mgn.

EE. Halteres capitulo toto vel fere toto lutescente.

Sp. 10. Clavipes Fabr.

DD. Scutellum nigrv-birtum.

Sp. 8. Bulbohum Radn.

CC. Thorax totus fulvo pilosus.

Sp. 9. Tuberculatus Rudn.

4A. Pedes partim testacei vel lutescentes, saltem basi tibiarum aut tarsorum.

F. Tarsi vel omnes vel aliqui toti nigri aut nigricantes, saltem supernc.

G. Pili verticis toti fulvi - Antenno nigræ, limbo infero articuli tertii intus non late sed distiocte luteo.

Sp. 13. Movtanus Mihi. 
Sp. 2 M. Subfasciatus Rndu. (Amali di Bologna 1845 ).

Similis iterum Cinereo Fabriciano, sed ab illo diversus Abdomine segnento tertio retro observalo vittis duabus transversis albicantibus distinguendis - Tarsis anticis et posticis fere tolis soperne nigricante-piceis, intermediis basi fusco-rufescenlibus. - Tibiis omui-

GG. Pili verticis postice fulvi vel latei, antice nigricantes $=A n-$ temne articulo tertio unicolore, vel nigricaute, vel piceo, rel sub-rufescenie.

11. Abdomen maculis et fasciis rufescentibus destitutnm; raro ad basim lateribus anguste rufis.

1. Tharax pilis fulvis vel luteis unicoloribus superne birtus.

h. Halteres capitulo fere toto albicante.

( maris $\cos x$ postice tuberculata non mucronat $)$.

Sp. 14. Submetallicus Mihi.

Ks. Ifatteres capitulo nigro, vel nigricante.

( Maris coxæ poslica mucronatæ).

L. Oculi superne nigricaute-pilosi, inferne pilis albicantibus.

T. Antenne articulo tertio rufo vel rufescente et bilongiore secundo,

$$
\text { Sp. 2. Subfasciatus Rodn. }
$$

TT. Antennce articulo tertio nigricante et trilongiore secundo.

$$
\text { Sp. } 4 \text { AENeus Mgrl. }
$$

LL. Oculi ctian superue albicante hirti.

$$
\text { Sp. 5. Eramius Mihi. }
$$

II. Thorax fascia transversa distineta pilorum nigrorum.

M. Abdominis segmentum ultimum tutum, ut pracedentes, fulvo pilosum.

Sp. 1. Punicue Beliardi.

MM. Abdominis segmentum ultimum, in mare fere totum, in licemina ad basim nigro-pilnsum. 
bus basi el apice, posticts anguslissime, rufescentibus. - Fascia pilorum nigrumm thoracis non manifesta, et abdomine prope apicem nou niggro hirto. Foeminæ speciei sergmenta tria abdominis albidi vittala.

Unicum exemplar mascuhun hujus merodontis caplum fuit in sicilia a D. Ghiliani et urihi transmissull a Marchione Spinola, et fominam a Prof. Bellatdi acceyi in alpibus captam.

III. Abdomen misculis vel fasciis rufesceatibus praditum, prater basales.

N. Fitke Irausverse albiantes abdominis, segmeotorum suturis subparnlela. - Vitta albicautes thoracis vel indistincta icl parum manifestx, tres intermedise el dux laterales.

\section{Sp. 16. Varius Radio.}

Ni. Vittre albicantes abdominis obliqua: Vitta thoracis semper maaifesta, dua intermedia et dua laterales.

\section{Sp. 17. Nigaransis Rudn.}

FF. Tursi omnes, saltem basi, rufescentes.

o Tarsi omnes omuino rufescenics.

P. Tibia omves vel aliqua in medio plus minusve late nigricanses.

Q. Antennce articulo tertio elougato-ovato.

Sp. 19. Spinipks Fabr.

QQ. Antemne: articulo tertio subcirculari.

Sp. 18. Sicanus Rudn.

PP. Tibire omues omnino rufescentes.

Sp. 20. Rufitious Rida.

ou. Terai at aficem nipricuntes. 
Sp. j. M. Cineneus Fabr. Mgn. Laeq. Rradn. (op. c.).

V. Descript. Meigenii et Macquartii.

Raro lectus in Pedemontio et mihi semel a D. Bellardi missus. Prope Tergestum quoque captus teste Schinero.

Sp. 4. M. Rxeus Mgrl. Mgn. Macq. Rndn. (op. c.).

V. Descript. Meigeuii et Macquartii.

Quibus adde, Oculi superne nigro hirti, infernesub albidi-pilosi. Foeminas esse credo speciri exemplaria abdomine salis obscuriore, et segmentis tribus transversim albidi vittalis.

Raro lectus in apennino Parmensi a Doct. Bertè et a meipso.

R. Abdomen lateribus ad basim rufis.

Suturis segmeutorum luteis vel albidis.

S. Abdomen ænescens $=$ Antennee articulo tertio etiam intus longiore precedente.

(Maris tibiæ postica appendicibus duabus validis ad apicem prædita. 1.

$$
\text { Sp. 7. Araupes Rado. }
$$

SS. Abdomen nigro-nitens $x$ Antennœ articulis secundo et tertio saltem intus aque longis.

( Maris tibiæ posticæ ad apicem apophisi brevi el tuberculo instructa).

$$
\text { Sp. 6. Mucronatus Mihi. }
$$

RR. Abdomen neque ad basim rufo-maculatum: suturis segmenturum nec albidis nec lutescentibus.

Sp. 13. Funestus Fabr. 
60

mensibus Julio at Augusto. In Pedemo ntio qunqueinu ventus a Prof. Bellarli.

Sp 5. M. Erarius Mihi.

Similis statura et habilu M. Eneo, tamen satis distinctus; pilis oculorum eliam superne albulis nun nigricantibus, et antennarum articulo tertio rufescente non nigro nec fusco-piceo.

Mares tantum lecti in apennino parmensi tempore ostivo, et olim cum maribus anei confusi.

Sp. 6. M. Mucroxatus Mihi. Sp. n.

Maris Longit Mill. 10 cisciter.

Nigro-nitens, pube lutescente, thoracis longiore, tectus. Thorax faseia unlla pilorum nigrorum - Abdomen segmento secundo maculis parris lateralibus rufesceutibus, fulvo-pilosis: tertio el quarto fasciola transversa, albicaute, suh-intermedia. - Halleres lutescentes Pedes femoribus apice angustissime, tibiis basi anguste, tarsis, apice excepto, fusco-rufescentibus. Tibire posticæ mucrone brovissimo apicali, et thherculo interiori prope apicem præditæ. Femora postica denticulo sub intermedio intus instructa - Coxce acuminato-productie.

Fixemplar uncum masculum in collectione D. Massino inreni, nescio si in Pedemolio vel in Liguria captum. 
Sp 7. M. Anupes Rudn. A. Costa.

V. Deseript. meam in Diariis, (Anuali di Bulogna 18:3) el (Magaziu de Zoolngie 18 t5).

Species ab cmmibus distineta facilius, appendicibus duabus validis, una moronata, altera compressa, ad apicem tibiarum, et tuberculo distincto in margine infero femorum, inter basim et prominentiam apicalem.

Sp Salis rama ter capta mense majo in agro-parmensi, bis in planitie aliera vice in collibus subapennimis, supra tores Euphorhiarmo et Chrysanthemi. - In Etruria $\left(^{*}\right)$ rguoque virit ubi prope. Pisam lecla fuit a Doct: Pechisili.

Sp. 8. M. Belborua Rindn. (Aunali di Bologna 18/3). Species cum nonunilis atictortun in unica conjunctis efformata, scilicet.

Nas. $\left\{\begin{array}{l}\text { Equestris Faim. Mgn. Marq. } \\ \text { Transversauts Pnz. Mgn. }\end{array}\right.$

Frem. Nobuls ifgn. Mlacq.

Sp. In Pedemontio lecía satis raro a Bellardi.

Sp. 9. M. Teibercuzatus Rndn. (Annali di Bologna 1845 ). Species instructa cum ununullis anctorum, riudes suut.

Narcissi Fabr. Myzr. Macq.

Constans liossi Hofg. Meig.

linavicans Fabr. Mleig.

(") Nuper iuventa elian frope Neapolim a Vuct. A. Custa. V. Dihrium G. Batr. Vıco 18:37. 
62

Sipreies rarn invenla in Pedemontio a Bellardi et ad Tergegesium a Schinero.

Sp. 10. H. Clayipes Fabr. Rossi. Latr. Mgn. Macq. Curtis Loëio. Rnàn. Walk.

Gravipes Rossi.

V. Descripl. Meigenii et Macquartii.

In collibus subapenninis agri parmensis legitur in floribus euphorbiarum non raro, presertim mense Majo, sed rarissimus in planitie, et tiltra junium nunquam inventus. Vivit quoque in Pedemontio et in Etruria testibus Bellardi, Pecchioli, et Rossio.

Sp. 11. M. Sexilis Mgn. Macq. Midn. (op. c.).

v. Descrift. Meigenii et Macquartii.

Fromina speciei sentel lecta in apennino parmensi a Doct. Rertèo.

Sp. 19. M. ItaLucus Rndn. (Annali di Bologna 1845).

Species condita pro duabus auctorum, inarem et fæminam sejungentium, seu

Mas. Melanguolices Fabr. Mgn. Mincq.

Form. Natars Fabr. Blgn.

Infrequens in ditione parmensi ubi legitur a mense majo ad angustum in floribus variis. 
Sp. 15. M. Mlontanus Mihi.

Foem. Lungit Mill. 12 circiter - Maris 10.

Form. Anternoe nigrex articu!o tertio subacuminsto, inferne intus lutei limbato.

Frons et Facirs ut Feriex toins, fulvo-pilosæ.

Corpus anen-nigrican: thoruce superne fulvo-hirto, pilis micoloribus; Scutelli unargine, pilis albilioribus.

Abdomen segmenti quarti lateribus, et quanto vel into vel lateribus et apice rutis: pilis ad basim albido flavidis, in medio fulvis, ad apicem refis hirtnm, segmentis tertio et quarto fusciola transrersa paulo albicante manifesta.

Pecies niggro-anescentes, tibiis basi anguste, el tarsis subtus rufescentibus.

Fominas duas legi metse angusto in apennimo Parmensi.

Mas rarissimo in l'edernontio lectus a Bellardi, in cujus collectione ascervitur - Hijus Ablomen ad apicen magis late rufun, at pili babules albidiores: pedes vero cexas non numcromatas prebent.

Sp. 14. 11. Subnetaldees Mihi. Maris Longil: Mill. 8-9.

Fusco-ienescena, basi ablominis 'ateribus macula rufescente. distincta, el incisuris, saltem in medio anguste alhidis, pilis fulvescentibus omnino techrs, excepto tanInm fasciculo verticali pilorum nigricantium. - Antonne, nigre, articulo tertio el basi ariste rufescenir-piceis, articulo secundo duplo circiter breviore tertia. - Petes nigrio andentes, bast tibiarma ge- 
niculisque testaceis: coxis posticis tuberculatis non muclenatis.

Slin nonine Enei distinetum, a Germania accepi, et nuper a Prof. Bellarli milui transmissum, prope Augustan Taurinorum inventum.

Sy. 13 M. Funestus Fubr. Mlgn. Macq. Rndn.

V. Descript. Mcigevii el Macquartii.

Species vulgaris in lola ditione parmensi et vulgatissima in planitie, ubi legitur in floribus præsertim ranunculoIrun mensibus Aprile Majo el Junio, rarissino in mense úlio el nor ulua.

Variat plurime colore pilorum et latitudine fasciarum.

Totam Italiam habilat. In Peỏemontio Bellardi, in Liguria Irussino, in Insubria Villa, in Eiruria Pecchioli, prope Mantuam D'Arco, prope Bononiam Bertoloni et prope Calaniam Gemellaro legerunt specient.

Sp. I6. Mi. Vasrus Kindn. (Annali di Bologna 1845).

Sp. bune distincta a congeneribus villis albidis obsoletis thoracis, pretep duas laierales, in ncdo dorsi tribus, et fasciolis albicantibus abuoninis, prxeserlim segmenti terlii, scibparallelis incisuris segmentorum, et non obliquis.

Variat pluriane colore abluminis et pedum.

Non infrequens in collibus sub-apenninis ditionis parmeu- 
si, a mense majo usqae au ociobrem. In planitie numquatu a me inventa.

Ls insula Meita exemplar foenineum obtinui, a D. Schembri.

Sp. 17. II Visattansis. Budn.

V. Descript. in diariss s. c. (Anbali di Bologna 1845).

Fon infraquas in agri parmensis planitie et collibus, præsprtiu neusibus Wajo el Junio. In Etruria quoque Inditat ubi lectus fuit a I). Pecchioli prope Pisam, et ad licora maris adriatici inventus teste Schinero.

Sp. 18. M. Siganve Indn. (Annali di Bologna 1845). Similis Spinipedi Fabricii, at duplo major et distinctissimus, masertim arliculo tertio antennarum subcirculari, non ovato elougato, nec subacuminato etc.

Obin specien institui pro exemplatibus drobus fomineis in Sicitia captis a D. Ghiliani et milı transmissis a Marchione spinola; pos evolgationen speciei novæe áleram foeminam obtinui ex insula melita a D. Schembri, et utyer etiam in agro pamensi exemplaria non sobma focminea sed eliam nascula inveni, quibus species nova confirmata est.

Miaris Abdomen tntum fulvum vel fere totum, et pube brevi stib-aturea tectum.

Scuteluan nigrricans, luteo vel fuivo-nilusum.

la descrip!ione hujus speciei jam evulgata, Scutellum dicitur 
66

luterm fulvo pilosum, sed erratum corrige, el lege Luei vel fulvi pilosum.

sp. Lecta won sape in floribus unbellatartum, presertin ancti fouiculi in collibus sub-apenninis parmensibus, sed in Sicilia et in Melila guorne a Ghiliani et Schembri inventa.

Sp. 19. Sphipes Fabr. Latr. Panz. Mlgn. Märq. Rudn. Ruficonnis Mgn. Macti.

Viaticus? Mrgn.

M. Ruficornem Mgn. et Macq. nunc cogito varietatem esse. - Spinipedis Fabriciani quia ab eodem diversum tantum colore antennarum satis vario, et absentia lineolartur albicantitu thoracis, quæ sepe parum manifestat aliquando obliteratæ sunt in Spinipede.

Species frequens est in agro parmensi, presertim mensibus Majo et Junio.

Sp. 20. Livitumis Rindn. Mas. Avrnus
fre. Prun

V. Neseriptiones Rossii et meam in diaris (Annali di Bologna $13 \% 3$.

Comunissimes in agri parmensis planilie et collibus a mense, aprile ad septembrem.

In lialia horeali et meridionali visil, ubi in Pedemontio legerumt Truqui et Bellardi, el in Sicilia Gemellarn. - 
In Italia media Rossius invenit, et duo sexus descripsit tarngivin speciebus diversis pertinentia. $\left(^{*}\right)$

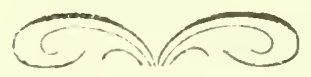

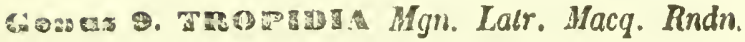
Bugerstatas Fall.

Chabact. Genehtci.

Antenner articulo tertio lato, extrinsecus subtruncato: arista dorsaali nuda.

(icnli uuli, in fronte maris colerentes.

Facies in medio carinata distincte.

Al 20 vera longitudinali quarta minus quam in generibus præcedentibas incurvata; quarta et quinta lougitudinalibus ad apicem conjunctis ante costalem.

I'cdes femortbus posticis crassis et apophisi valida apicali præditis.

(*) $5 p$. Albifrons $\mathrm{Mg}_{\mathrm{g}}$. nisi aliarum varietas est, Italicis ahtenda, nan teste Schinero, prope Tergestum vivit. Vide Bescript. in opere Moigenii. 
Observat. et Synon.

Sp. 1. T. Fasciata Mrgn. Macq. Rndn.

v. Descript. Meigenii et Macquartii.

Exemplar unicum masculum speciei olim a D. Gendo accepi absque patriæ indicatione. An Pedemontana?

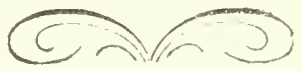

Stirips V. SYRPHIN E Rndn.

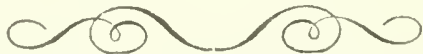

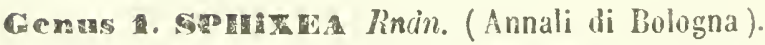
Fareaga Latr. Fab. Blgn Macq. St-Farg.

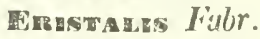

Syreptes Rossi.

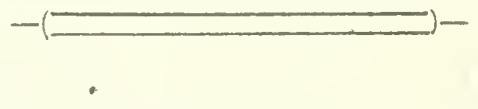

Cuallact. Generici.

Antenne articulo tertio subcirculari, arista dorsuaii nuda. Oculi nudi, in froute maris anguste contigni. 
Trons modice et won satis producta.

Ale Venis !ongitudinalibus secunda et tertia conjunctis ad apicem ante costalem: ultima et peaultima conjunctis ante marginern posticum, el ultra conjunctionem earum, appendice venosa slatim flexa, et margiuem petente: transversaria exteriore satis obliqua et valde proxima apici longitudinalis sextæ, et distante a transversaria intermedia.

Pedes femoribus posticis nec distincte incrassatis nec inferue serrulatis, sed prope apicem denticulo distincto, iufero præditis. Abdomen prope basim haud manifeste coarctatum.

Species ltalice.

4. Ablomen nigri et flavi fasciatum.

Sp. 1. Splendida Rossi.

A 1. Abdomen fertuginei et llavi fasciatum.

Sp. 2. Crabronifonyis Fab.

Observ. et Synonim.

Sp. 1. S. Sple muida Rossi (1800).

Fuluivate Fabr. (1805) Mgn. Macq. Rndn.

V. Descriptionem Rossii.

In planilie parmensi raro, in collibus et montuosis infreqtenter legitur, a mense Junio usque ad Seplembrem. 
In Etruria Rossius et Pecchioli, in Sicilia D. Costa Sy. invenierunt, et ad Tergestum Schiner.

Sp. 2. S. Conabronionais Fabr. Latr. Mgn. Mactq.

\section{Gigas Rossi.}

V. Descript. Rossi et Meigenii.

Sp. sat rara: una vice tantum a me capta in apormino parmensi et altera vice a Doct. Berteo. In Pedemontio quoque et in Sicilia habitat, ex quibus regionibus exemplar míli transmissum fuit a Prof. Benlardi, et Gemellaro.

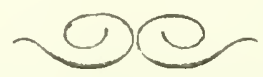

Genus ar. Matha Latr. Fabr. Mgn. Macq. Zelt. Rndn. Szapes Rossi. Panz.

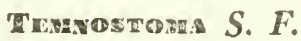

Spuromin Iign.

Mesca Lin.

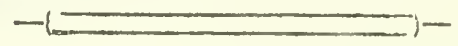

Characteres Genenici.

Frons, Antenne, Oculi, Abdomen, Pedes, circiter ut in Gen: Sphixea; sed Alce venis longitudinalibus secunda et tertia sejunctim cosialem a:tingentibus, et appendice venosa ultra conjunetionens 14 th ma 
et pasultim loogitudinalium, satis decurrante margini postico subparallelit:r untequam ipso conjangatur (1).

Spec. Italica 1. Sazteua Fibr.

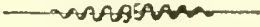

Observat. et Synon.

\$p. I. M. Saltudr Falr. M'gn. Zelt. Rndm.

Dioputilula Lin. Rossi. Falir. Latr. Fall. Panz. Mac!.

V. Descript Zetterstedtii.

Frequns in agro parmensi a Junio ad Septembrem, sed in phanilie mints commnis,

Exemphr sicuhm a March. Spinola, etrussur a Pechioli iccepi (2).

(1) Nonen Genericum Spllowya Mgn. prospecie Tespiformi (non Italic.) retineudum, cum Dimpthalmu a Meirenio conjuncta; sed ninc separanda; et spec. Diphlhalma in MLLEsis cum $\mathrm{S}_{\mathrm{P}}$. Stluzun locum habebit.

Teurostons nmen genericum a $S$. Fargeau propositum non adoptandum, quia jam Spromye genus pro specic ejusdem typica institutun. crat a Meigenio.

(2) Sp. Diophtalme. Irigenii, uondum in itaila capta huie gener refapeda. 


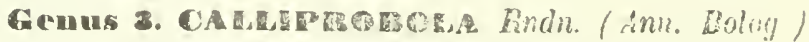
RILesa Fabr. Latr. Fgn. Facq. Zett. Suapeaso Rossi. Panz.

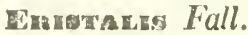
RJEGA Lin.

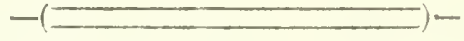

Charact. Geneli.

Antenna, Arista, Oculi, circiter ut in geueribus pracedintibus sed. Frons magis et satis producta. - Facies non tuberculata.

Ala renis longitudinalibus secunda el tertia sejucuctim cusialen it:tingentibus: appendice venosa ad conjunctionen longiiditualium ultima et penultimæ, brevi subrecta et statim nargau postico producla.

Pedes femoribus posticis denticulo infero destilula. Abdomen prope basin non coarctatum.

Species Italic.e.

A. Abdomen ænescens, segmentis posice pilis ulvis marginatis.

Sp. 1. Specioa liossi.

AA. Abdomen nigrum, segmentis duobus apcahbus ferrugineis.

$$
\text { Sp. 2. TALLA Lim. }
$$


Observat: et Synonimia

Sp. 1 C. Speciosa Rossi. Lalr. Fabr. Panz. Mgr. Mlacq. Curtis Indn. Wall.

v. Deseript. Rossii et Meigenii.

Rara in ditionis parmensis planitie et collbus, mensibus aprile et majo, in fioribus arbtstorum,

In Etruria quoque habitat ubi prins a Rossio lecta, et prope tergestum capıa a Doct. Schiner.

Sp. 2. C. Fallax Lin. Fabr. Mgn. Macq. Zelt. Semirufa Fabr. Fall. Vide Descript: Zetterstedtii.

Apud nos rarissima, in Pedemontio huc usque tantum invenla a Prof. Bellardi.

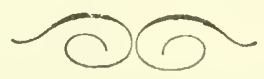

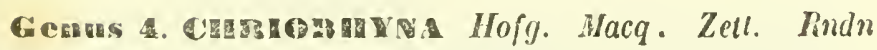
Walk.

MEIEEsa Mign.

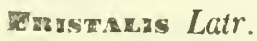

Sviremes Fall.

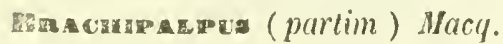

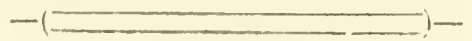


Charict, Genentc.

Antenne arliculo lertio longitudate laciore, raro subsoluddato; arista dorsuali nuda.

Facies in medio luberculara; Enisionio iuferne magis vel minus sed distiucie semper froducto.

Oculi nudi, in fioute angustissine subcontigui.

Abdomon prope basim non angustalum.

Ale ut iu Calliprobala venosa.

Pedes robusti sed lemoribus uullis nec satis incrassatis nee infera deaticulatis nec spinulosis; coxis et thib non tuberculatis etc

\section{Species Italic.}

A. Tiorax pilis nigris omnino lectus.

Sp. 1. Rinuxceu Panz.

A T. Thorax vel in parte anteriori tantum, vel ubique fulropilosus.

B. Tarsi toti nigri, vel piceo-nigricantes.

C. Thorax antice pilis rufis vel fulvis, postice nigris, hit?us.

$$
\text { Sp. 2. Aplc.tтd Mgn. }
$$

C.. Thorax pilis rufis vel luteis ommino tectus.

D. Sculdlum pilis nigris liirtum.

$$
\text { Sp. ว. Hexsenta Fabr. }
$$

D). Scrotellum pilis rafis vel fulvis himun.

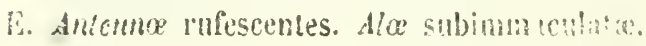


F. Corpus totum pilis rufis tecturn.

Sp. 4. Brebssonu hiacq.

IF. Thorax lateribus, abdomen apice, pilis albido-lutescentibus, alibi pilis rufis vel fulvis lirita.

Sp. 5. Oxyachanthe MIgn.

EE. Antennce nigricantes. Ala magis vel ninus distincte fusçu-maculatæ.

Sp. 6 Floccosa MIgr.

BB. Tarsi vel toti vel saltem late ad basim lutescentes.

Sp. 7. Femorata Lin.

Obsercat. et Synonim.

Sp. 1. C. Ranuxcul Panz. Mgn. Walk.

V. Descript. Meigenii.

Apud nos in Pedemontio tantum lecta sat raro a Pr. Bellärdi.

Sp. 2. C. Apigata Mgn. Macq.

v. Descript. Meigeuii et Macquartii.

Rara in collibus subapenninis dilionis parmensis ubi mense aprile exemplaria nonnulla legi uiliusqque sexus in floribus buxi. 
Sp. J. C. Berserixa Fubr. Mqu. Hacq. Löew. Zett. Walk. Vide Deseript. Znterentritii.

Unicum exemplar speciei in culfectione mea asservalum Pr. Geneus milhi commenicavit.

An Pedemontan!n!?

Sp. 4. C. Bufbissonu Macq.

V. Deseript. Macquartii.

Exemplaria duo speciei fominea in collectione mea adsunt, unun a me capiun in apennino parmensi, alterun in Pedemontio a Bellardi.

Dubito speciem hanc varietatem esse Chr. Oxyachunthæ, pilis totis rufis.

Sp. 5 C. Oxyachanthe Mgn. Walk. non Macquari, quia non similis Berberine, ut dicil Cl. anct:

v. Descript, Mteigenii.

Raro lecta in apennino parmensi a D. Bertéo, et in Pedemontio a Prof. Bellardi.

Sp. 6. C. Foccosd Hlgn. Macq. Rndn.

v. Descript, Meigenii et Macquartii.

Fomina speciei bis lecta in apennino ditionis parmensis a Doct. Bertèo. 
Sp. 7. C. Femorata Kì. Fal! MLin. (non Hacy. nec Zett.) Ialli. (exlisa syton: Volvulus Fabr. et Mign.)

A.MForigs Bacy.

Fonina spec: tantum capta a me prope Parman scmel:

narem in pedemontio in rentun Bellardi misit.

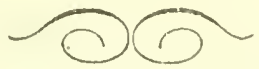

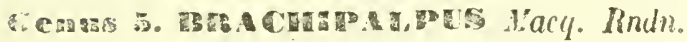

TSTATA Mgn. Zell.

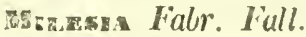

Nörsca Lin.

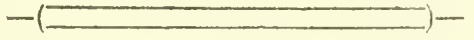

Charact. Gener.

Antenne articulo terlio trausversim ovato; arista dorsuali nuda.

Oculi nudi, in fronte maris antice satis approximati sed angustissime? et non coutigui.

Facies supra epistomiun produetum, non gibbosa, sed excavata. Abdomen prope basim nou coarctitum.

Alex ut in Calliprobola circiter venosa.

Pedes femoribus posticiz distincte incrassatis, inferne bisertintim serrulitis: Thibiis pasticis prope basim tuberculatis. 
Species Italic.s.

A. Tarsi antici et postici toli nignri vel nigricantes.

Sp. 1. Olvaceus Wiedm.

AA. Tarsi antici el postici ad basim magis vel minus late rufescentes.

Sp. 2. Valgus Panz.

Observationes et Synonimia

Sp. 1. B. Olivaceus Wiedm. MIgn. Rndn. (Gener. Ital.) Thernculatus Macq. (Fœm.) Rndn. (ordinam sistem;. v. Descrip. Meigenii

- Homina fomora postica extrinsecus rufescentia et partia piam interitus: intermedia fere tota rufa.

Non fregitiens in ditione parmensi, mbi mares nomnullos les:, sed rarissimo fominam. In floribus Pruni Padi et rinunculorian eos inveni, mensibus martio et aprile. Tariat femoribus eiam ima basi nigris.

Sp. 2. B. Valgus Panz. Mgn. Zell. Macq. ( sed exclu Synon: Femoratus Fabr. et Fall.)

Lafirilomis Fall.

1. Descrį̣t. Zettersteåtii.

Fonina ane reperta, larsos omies prebel etiam apice fulvos. 
Rarissimo a me inventus $m$ : in foribns ranunculorum mornse martio, in planilic propejadana parmensi, fominam seaiel lrgi.

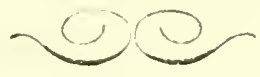

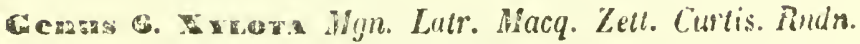
likitiv.

Igrgaesa Falor. Latr. Iall.

Nizentersossi. Panz.

ricera I.in.

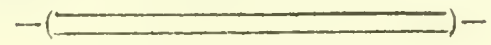

Chanacteos Genenici.

Anlenne articulo terlıo subcirculari vel breviter ovatı, arista dorsuali nuủa.

Oculi wull, in fronte maris magis vel minus late contigui.

Facies excavata supra epistomium, non distincte productum, ast partin.

Ablomen prope basim non coarctatum.

Seutellam margine non serrulato nec grandoso.

Alle ut in Chriorhyna circiter venose; vena transuresa cxteriore magis distante ab mleriori quam ab apice longitudimatis spzlm, inl fere argudistanle.

Pedls femoribus posticis distincte incrassatis, inferne scriatim spinalasis: (ibiis mon tuberculatis. 
Species Italigk.

A. Abdomen maculis et fasciis luteis vel rufis nullis.

B Audomen apice aureo-tonentosum. Tursi postici lutescentes, apice nigricante.

$$
\text { Sp. 1. Sycvanum Lin. }
$$

B13. Abdomen tomento aureo apicali non observando. Tarsi postici nigricantes fulvo tomentosi.

$$
\text { Sp. 2. Abiens Wiedm. }
$$

Ad. Abdomen fulvi vel rufi vel rubescentis fasciatum, aut maculatum.

C. Abdomen maculis quatuor fulvescentibus signatum.

D. Macula abdominales subrotundatæ vel subtrigonæ. Femora postica parum incrassata.

$$
\text { Sp. 5. Neronuss Fabr. }
$$

10. Maculo abdominales subquatratæ: - Femora postira salis incrassata.

Sp. 4. Fonum Fabr.

C.C. Abdome in medo late fulrescens vel rubescens.

R. Tirsi non ombino nigri, partim lutescentes.

l. Tibic anticæ et intermediæ lutescentes, annulu fusco.

Sp. 5. SEgNis Lin.

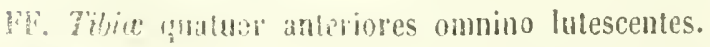

$$
\text { Sp. 6. Ignuy Panz. }
$$


EE. Tarsi omnino nigri.

Sp. T. LeNTa Mgn.

Observat: et Synonimia

Sp. 1. X. Srevanum Lin. Fabr. Mgn. Latr. Macq. Zott. Walk. Inpiger Rossi Panz.

Vide Descript. Zetterstedtii.

Rin frequens in agri parmensis collibus et montibus etiam elatioribus.

In Etruria etiam vivit teste Rossio, et in Pedemontio a BelIardi in Liguria a Mussino in alpibus Insubriæ, a Villa lectus, et ad Tergestum a Doct. Schinero.

e. 2. X. Asiens. Wiedm. Mgn.

Coeruleiventris Zelt.

v. Descript. Meigenii.

Snecies satis rara, in Pedemontio lecta a Prof. Bellardi.

s.p. 3. X. Nenonuz. Iabr. Mgn. Zetl. Loëw. Walk. (non Macq. (quia Tibice in hac specje nigricante annulatic.)

Iginava Fall.

Vide Descript: Zellerstedtii.

Tarissimo a me lecta in collibus subapenninis ditionis parmentis. 
5p. 4. X. Flonum. Fabr. Wiedm. Mgn. Maoq Zelt. Bifasciata Curtis Walk.

V. Descript Zelterstedtii.

Mas speciei a me semel captus in collibus agri partuensis, foeminam semel accepi a Bellardi, in Pedentontio ab eoden inventam.

Sp. Э. X. Segins. Lin. Fabr. Rossi. Latr. Fall. Panz. Mgn. Macq. Loëw, Zett. Kndn. Walk.

v. Descript, Zctterstedtii.

Vulgaris in lota ditione Parmensi, a mense martio usque ad octobrem. - In aliis eliam italiæ regionibus habitat, ab Alpibus pedemontanis usque ad Siciliam, ut exemplaria mihi transmissa a Bellardi, Mussino, Villa, Pecchioli, D'Aico, Costa confirmant.

Sp. 6. X. Ignava. Panz. Mgn. Máaq. Zett. (non Fall.)

V. Descript. Zellerstedtii

Sp. raro a me capta in ditionis parmensis collibus, mense majo.

Sp. 7. X. Lexta Mgn. Macq. Zett.

Pigra Panz. Fall.

V. Descript. Zelterstediti.

Non infrequens in locis montuosis et collinis agri parmensis, in floribus præsertim corni sanguinei legendus, 
mense majo. In pedemonlio quoque frequens videtur, nam ejusdem exemplaria sæe obtinui a Geneo, Truquii, Bellardi, et Mussino, etiam ad Tergestum ut Schinerius affirmat, et in Etruria a Piccioli inventa.

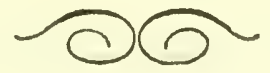

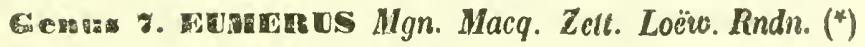
Walk.

syrepues Fabr. Rossi. Panz.

Pipiza Fall.

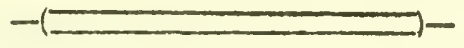

Cuaract. Generict.

Antenno articulo tertio vel subcirculari vel ovato, vel apise sabtruncato vel paulo acuminato. Arista dorsuali auda.

Oculi, saltem in mare, pilosuli vol puberuli, in oodem sexu contigui, vel subcontigui, nunc magis nune minus late, (rarissimo panlo remoti.)

Facies plana, supra epistomium non productum, nec tuberculata, nec cariuata, nec excavata.

Scutcllum margine breviter serrulato vel granuloso.

Abdomen prope basim nou coarctatum.

Alir, vena quiara longitudinali bicubitata, seilicet ultra cubitum ordinarimm valde intus tlexa et sic angulos duos efficiente, api-

(1) Species Italic Generis Euneri (Annal. Societ. Eatomol. Pai.sirusis 1850 ;. 
cihus angulorum appeudicalatis: veuula iranstersa ezterior magis proxima apicl sexta tongitudinalis quan transverse iuteriori.

Pedes, femoribus posticis plus minusve incrassatis vel sallem paulo crassioribus, inferne magis vel minus distincle serrulatis.

Species Italiez.

A. Abdomen dorso magis vel minus late et distincte rubescente.

B. Oculi non manifeste pilosuli.

sp. 1. Sabulonum Fall.

B. Oculi manifeste pilosuli.

C. Intennce articulo Ђ.॰ ad apiccm inciso, inferne non acuminato. - Abdomen segmentis ultinis dorso haud niveo pubescentibus.

Sp. 2. Tricoton Fatr.

CC. Antenne articulo $50^{\circ}$ apice non inciso, inferne subacuminato. - Abdomen segmentis ultimis, certa luce observatis, dorso nivei pubescentibus.

$$
\text { Sp. ว. Brcolon Bellardi. }
$$

AA. Abdomen dorso non rubescente.

D. Pedes femoribus posticts vix crassiusculis. - tibiis et tarsis posticis pube nivea in fomina, sub argentea in mare, superne tectis omnino.

Sp. '. Exiupes Krdn. 
DD. Pedés femoribus posticis nagis vel minus sed distincte crassis - Tibiis carsisque posticis etiamsi pau!o pilosulis, tamen pube nivea et argentea non teclis superne.

d. Pedes omnino atri, etiam ad basim tibiarum.

Sp. 5. Meunopes Mihi.

dd. Pedes non omnino nigri, saltem basi tibiurum sordide testacea.

T. Oculi in fronte inaris late coherentes, fominze non distantes ut in aliis fæeminis. - Arista in utroqne sexu propius apici articuli inserta. - Fomora postica paruin incrassata.

Sp. 6. Ornatas Mgn.

EE. Oculi in fronte maris magis vel minus anguste contigui, in foemina distantes ut in aliis fœminis. Arista in utroque sexu propius basi articulì inserta. - Eemora postica satis aut valde incrassata.

F. Tarsi quatuor anteriores nigri vel nigricantes saltem ad articulns apicales.

G. Cox quatuor anteriores manifeste, et totx, aut late rufescentes.

II. Abdomen in mare penicillo ventrali pilorum alborum præditum, in utroque sexu segmento ultimo duplo circiter longiore præcedente. - Tibiæ posticæ in mare unco apicali intus instruetæ.

Sp. 7. Uxcipes Rndn.

HII. Abromen maris non penicillatum, utriusque sexus segmentis duobus lillimis longitudine parum diversa. 
lateribus in mare longe barbatis. - Tibice postica maris unco apicali destitutæ. - Femora postica in eodem sexu longe barbata.

\section{Sp. 8. Barbiventris Iindn.}

GG. Coxa quatuor anteriores nigricantes, vel summo apice tantum parum rufescente.

1. Femora postica fere ut in genere Merodonte, ad apicem, apophisi infera valida, instructa.

Sp. 9. Alpinus Bellardi.

II. Femora postica prominentia subapicali valida infera destituta.

K. Anterno articule tertio aut toto nigro vel nigricante pieeo, aut saltem fusco-rufescente, limbo vel apice nigricante.

L. Antennæ articulo tertio amplissinı, (foeminæ) antice rotundato. - Alce rena quinta longitudinali in parte - ubitata appendiculis tribus instructa, duabus exterioribus, una interiori.

\section{Sp. 10. Funeralis Mgn.}

LL. Antenna articulo tertio modice lato, vel apice obtruncato. - Al $\propto$ rena quinta longitudinali in parte cubitata appendiculis duabus tantum exterioribus prædita.

M. Femora postica prope basim inferne non tuberculata.

N. Oouli crebre et longe pilosi. - Antenna nigræ vel piceonigricantes. 
NN. Oewli parce et brevissime pilosi. - Antenne fusco subrufescentes in mare; rufescentes, limbo vel apicis nigricante in fremina.

Sp. 12. Strigavis íall.

114. Femora posica luberculo infero prope basim instructa, (cum prominentia coxarum proxima non confundendo)

Sp. 13. Tuberculates Mihi.

KK. Antennec articnto tertio to to fulvo vel testaceo-fulvescents.

O. Tibice posticat valde dilatato-clavatæ, intus canaliculs: !ongitudinali distinctissimo. - Antennæ arista prop apicem articuli tertii inserta.

Sp. 1/. Clavitibius Rndn.

OO. Tibia posticæ modice seu non ralde dilatatæ, nee inIIIs distincte canaliculatæ. - Antenne arista in medio dorsi nisi propius basi articuli tertii sita.

f. Oculi manifeste pilosi.

Sp. 13. Delicate Schembri

ff. Oculi non manifeste pilosi.

Sp. 16. Lesops Milli.

FF. Tarsi qualuor anteriones rufescentes vel fulves centiw raro articulo permltimo tantum panlo infuscato.

P. Abrlomen nigrum. - Antenne maris articulo tertio angrusio, elongato-suhovato, fuemina late ovato.

Sp. 17. Angtsticonists Rindn. 
PP. Abdomen euprei vel anei coloris. - Antenna utritsque sexus breviter et late sub-ovatæ.

Q. Intenna vigricantes, saltem articulis duobus prim is et limbo lato tertii nigris.

$$
\text { Sp. 18. Barbarus Wiedm. }
$$

$Q Q$. Anternce omnino fulvæ.

Sp. 19. TruQvil Mihi.

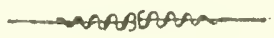

Observat. et Synon.

Sp. 1. E. Saвmroxum. Fall. Zett. Rndn. /Annal. Soc. Ent. paris 1850).

Rubriventris Macq. Meig.

v. Descript. Zetlerstedtii.

Species raro capta in apennino el collibus agri parmensis a Doct. Bertèo et a me, mensibus Junio et Julio.

Sp. 2. E. Tnicolor. Fabr. Latr. Mgn. Macq. Rndn. Mixtus Panz.

v. Descripl, Zelterstedtii.

Speciem non frequenter legimus cum Doct. Bertéo in Apennino, sed in collibus etiam parmensibus ego inveni sat raro.

Ex insubria (forte montana) ejısdem exemplar olim accepi a De Cristofori, et ad Tergestum inventus a Schinero. 
Sp. 5. E. Bicolon Bellardi Sp. n. (In litieris).

\section{Longit Mill. 7 circiter.}

(Mas) similis luabitu et statura Eumero Tricolori, at paulo majol et distinctissimus.

1. Antermis articulo tertio apice non inciso sed inferne subacmminato.

2. Oculis in fronte subcontignis et non paulo distantibus.

5.'Abdomine lun!lis albidis non distinguendis.

4. Femoribus posticis minus incrassatis.

5.D Segmentis tertio et sequentibus abdomiualibus pube Jorsali nivea.

A. Pedibus pilis albidis instructis et non tantum pubescentibus.

Ceteri characteres circiter Ht E. Tricolore, scilicet Epistomium ut frons antice, n!grum albiti pilosum. - Vertex nigronitens nigricante pilosus. - Antenne nigræ. - Thorax niger, nigro pubescens. - Calipera It halleres albicantia. - Abdomen segmentis secundn et tertio lateribus rubris, inferne totis rubidis. - Pedes nigri, geniculis et ima basi tibiarum fusco Iniescentibus.

Spec. Typica in collectione Bellardi allest ab eodem in alpibus pedemontanis capta.

Sp. \&. E. Exulupes Rndn.

v. Deseript. in Disriss. (Annal. Seiet. Entumol. Gallix 18ü0.)

Species ab ommibus distincta juesertim. Femoribus satis exilibus, el tibiis tarsisque pesticis in mare pube nited tecis, in iemina rebre alumblobs. 
Oculi maris in fronte lale cohernles.

Non rara præsertim in colibuti sthapenninis dilionis Parmensis, ubi legilur mensious majo et junio, et rarius Julio.

Sp. 3. E. Melanopus Mihi. Long. Mill. 6. fam: 7. mas.

Antennce (maris) nigræa albo-nitentes: arliculo tertio ovato; arista in medio circiter dorsi sita. - Facies alba, albo-pilosa. - Frons anlice sululutesrens, pilis pallide luteis. - Oculi pilosuli, in fronte anguste conligui. - Pedes toli nigri etiam basi tarsormm el tibiarum, albi pubescentes.

Catera "It in mare (circuter) E. Strigati.

In fomina facios el frons nigrn-œnesceutes, parce alhi pilosnlx.

Antenno minus nigra, sed cxtera ut in mare

Semel exemplar masculum tempore æslivo in collibus ditionis parmensis inveni. - Fæminan in Etruria captam. a D. Piccioli accepi.

Sp. G. E Ornatus Mgn. Macq. Zetl. Kindn. Walk. Fugirivus Rossi.

V, Descrip: Zelterstedtii el ubservat, meas in Diariis sup̣rn citatis $\mathbf{1 8 5 0}$.

Non infrequens a mense martio usque ad septembrem in apenuino collibus et planitie ditionis parmensis. Prope Tergesium a Schinero cinorque inventus. 
$S p$. 7. E. UNCIPES Rndn.

V. Descript. meam in diariis s. c. 1850.

Non vulgaris sed non rarus in collibus agri parmensis a mense majo ad septembrem.

Sp. 8. E. Barbiventris Rndn.

V. Descript. meam in diariis s. c.

Non frequens in agro parmensi, priesertim in collibus subapenninis, mensibus Junio et Julio legendus. In Apennino mense Augusto inventus.

Sp. 9. E. Alpinus Bellardi $S p . n$. (in litteris ).

Longit Mill. 7. eirciter

Cinnibus nostratibus majur. Nigro-œnescens, abdomine nigriore.

Antennce nigræx, articulo ultimo sub-ovato. - Caput, epistomio et fronte antice albido lutescentibus et albopilosis, vertice nigro-œnescente et nigro piloso, antice fasciculo pilorum albicantium distincto. - Oculi superne pilosuli, in fronte modice late coherentes, orbitis posticis albidis. - Thorax nigro-pubescens, albiditrivittatus, vittis ultra medium dursi postice produ ctis, intermedia exiliori, lateralibus presertim antice latioribus. - Abdomen dorso nigropubescente, viltis transversis ordinariis albicantibus, lateribus et supr:a villas albillas pallide lutei pilosun. - Pedeș sordide luten-pilosi et pubescentes, tibiis basi angustr ritre- 
scentibus, fenoribus posteris satis crassis, ad aprce. inferme apophisi validd et suhtus spinulosa preditis. - Tarsis incrassalis el duplo circilep brevinribus tibiis. - Calipter ut hulleres alhicantia. - Alco griseæ, vilta styģmaticali nigricante.

Marem speciei in alpibus pedemontänis capttim semel mithi Prof. Bellardi misit.

$S_{p}$ i0 E. Funeralis Mgn. Macq. Loëw. Zelt. Zindn.

V. Descripl. Zetters!edtti.

Semel rantum exemplar focminem speciri a me lccun fuit in collibus ditionis Parmensis mense majo.

Ista ab aliis distincta latundine magna articuli tertii antennarum, atri et subcireularis, sed eliam vena quinta Inngitedinali alarum in parte cubitata præter appendiculas duas ordinarias exteriores, tertia appendice interiori prædita. - Si character iste alarum constans est in specie, facile et optime ab onnibus aliis distinguenda.

An species diversa?

Sp. 11. E. Lastops Mihi. Longit Mill. 6-7.

Habita et slatura Eumero strigato Fallenii similis, tanten distinctus, antennis in fornina partim rufis et paulo rufescentibus in mare; et oculis magis crebre et longius pilosis Similis quoque E. Tisbereulato meo (N, 10). sed major, et femoribus ad hasin inferne non tuberculatis. 
Non frequens in agri parmensis planilie et locis monluosis, a mense majo ad septembrem.

Sp. 12. E. Strigatus Fall. Mgn. Macq. Zetl. Rndn. Walk. v. Descript. Meigenii et Zetterstedtii.

Non infrequens in planilie el collibus dilionis parmensis, ubi eun inveni a Junio al septembrem.

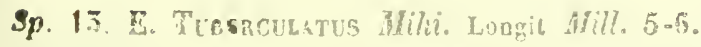

Specing similis E. Strigato et Lasiopsidi sed ab islis et ab boumibus distincta, tuberenio fomorun posticorum in parte infera mope basion observanto, et cum prominen'ia coxalun proximarmon non confundendo.

Coeteri characteres, quibus ab unis vel ab aliis Eumeris divessus apparet sunt.

Loxgitudo Mil. \$-6.

(Ulerque sexus) Nigro cenesccus, pallide lutei puberulus, præsertiul lateribus el apice abdominis. - Thor ax viltis duitbus albicantibus vix aut párum manifestis. - Pedes basi tibiarum et tarsorum magis vel minus late et distincte rufescente - Femora salis crassa.

(Maris) Antenno nigricante piceæ, snb-ovalæ, apicesub-obtrunsatie. - Oculi puberuli, in fronte modice late coherentes. - Epistomium ut pars antica frontis, also-pilosum. - Vertex nigro pilosus.

(Foem.) Antennce articolo tertio majori subcirculari-ovato. Frons latiuseula, nigro menescens, albidi puberula, et 
piope oculos albidior. - Oculi minus manifeste pubescentes.

Bis marem et foeminam speciei inreni cum pracedentibus, iisdem temporibus et locis, et olim cum E Strigato istius exemplaria confusa.

Sp. 14. E. Ceavitibius Rindn.

V. Descript. meam in diariis Societ. Ent. Galliæ. 1860.

cui adde, Antennce etiam articulis primis fulvis.

Arisla prope apicem articuli terti antennarum inserta, et libice posticæ satis incrassato-clavatæ, et canaliculatæ in parte interiori, et sic ab aliis Eumeris bene et facile distinctus est.

Bis marem inveni in collibus subapenninis agri parmensis.

Sp. 15. E. Deucate Schembri (in Litteris) Rindn.

V. Descript. neam in diariis s. c.

Species, in genere, minima, vix Mill. 4-5 longa, quo charactere parvitatis, aliis adjuncto, ab illiis vel ab istis congenerum bene distincta videtur.

Frequens in insulit Melita teste D. Schembri, et a me semel lecta fœmina in collibus subapennis parmensibus, quæ difert a maribus melitensibus, colore basis antennarum in illis nigricante in ista fulvescente.

Sp. 16. E. Lesops Mihi. Longit. Mill. 5-6 (mas).

Anlennce testacex, articulo ullimo sub-ovato, aristam in 
medio circiter dorsi ferente, articulo primo et arista nigris. - Ocull non manifeste pilosi, in fronte anguste contigni.

Coetera ut in E. Strigato (cirriter).

Marem ter legi mense septembre in collibus sub-apenninis parmensibus, et alterum ab Etruria D. Piecioli misit.

Sp. 17. E. Angusticornis $\boldsymbol{R} n d \boldsymbol{n}$.

V. Deseript. meam in Dlariis s. c.

cui adde Abdomen maris segmento anali satis incrassato.

Frequens in ditionis parmensis collibus, a mense Junio ad septembrem, in floribus umbellatarnm, et prasertim Danci Carotæ.

Sp. 18. E. Barbarus Wiedm. Kndn.

V. Descriptiones Wiedmani et meam in diariis s. c.

In insuta Melita a D. Schembri non raro inventus, a quo nonnulla speciei exemplaria utriusque sexus accepi. Individua a Wiedmanio descripta in Africa boreali lecta luerunt.

sp. 19. E. Treque Mihi.

$$
\text { Longat Hill. 10-11. }
$$

Antenne numino fulve, articulo tertio breviter ovalo, arista in torso articuli tertii, magis longe ab apice ynam a basi inserla. 
$\$ 6$

Caput, rpistomio el pronte antice albidis el albo pilosis, vertice oeneo linea antica Iransversa pilorum nigricautium, postice pilis Intescentibus hirto.

Thorax ilorso oneo stb-aureo ut sculellum: vittis duabus donsualibus el pube, at latera longiore, albidis; pleuris nigro aneis albidi pilois.

Abdomen lateribus el apice aneis, dorso cupren, lunulis albicantibus transversis albidis in medio conjunctis, non divisis ut in aliis speciebus.

Pedes nigro-mescentes, albido pilosi, tibiis fulvis annulo fusco, tarsis omnibus rufescentibus, coxis, præscrtim intermediis paulo rufis.

Alce in inedietate basali fusco lutescentes.

Semel mas speciei ab Equite Truqui missus in pedemontio captus.

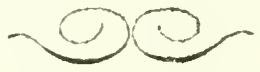

4ienus 3. X Yatatera Rndu. (Amal. Bonon. 1844). דхтота Mgn. Macq. Zetl.

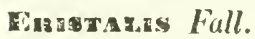

Truessey Fabr.

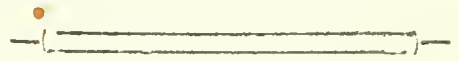

Chanacteres Geverici.

Autrome articulo tertio sohtentieulari, arista uuda, dorcuali, prope basin arliculi imsirta. 
Facies in medio excavata, et supra epistomium, sultem in mare, tu. berculata.

Oculi nudi, in fronte maris late colserentes, Abdomer prope basim non coarctatum.

Ala venis longitudinalibus quarta et quinta, prope apicem in uniea confusis, et simul costali productis: venula transversaria exteriori magis proxima interiori, quam apici longitudinalis sextæ, ubi conjungitur quinta.

Pedes femoribus posticis, haud satis incrassatis, omnibus in parle apicali stblus serrulatis.

Species Italice.

A. Abdomen omnino nigro-suboneum.

$$
\text { Sp. 1. Vara Fitir. }
$$

AA. Abdomen lateribus magis vel minus late lutescentikus. Sp. 2. Dubia Fubr.

Observ. et Synomim.

Sp. 1. X. VARA Fabr. Mgn. Inndn.

v. Descript. Heigenii.

Non infrequens in agro parmensi: supra fiores varios arbustorum, sed etiam eıphorbiarum legitur, mensibus 
98

prasertim aprile el majo, rarius junio, el non ulira euru inveni.

Sp. 2. X. Dubia Fabr.

Lateralis Fall. Mgn. Macq. Zell.

จ. Descript. Zetterstedtii.

Non tara in ditione parmensi tam in planilie quam in montıosis, eosdem flores diligit quos alterá species frequentat. - Eam legi mensibus majo et junio. Exemplar unum accepi ejuside m a Doct. Costa, in Regno neapolitano captum, alterum ab Etruria a D. Piccioli, et aliqua a D. Schiner inventa ad Tergest um.

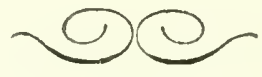

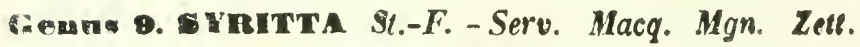
Rndn. Walk.

Xxioxa Mgn. Latr.

Huessia Fabr. Latr. Full.

Exnencs Fabr. Rossi. Pans.

Mesca Lin.

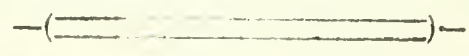

Chabact. Grimer.

fntemus arliculo tertio subcirculari, arieta dorous li auda, prope besim articuli inserta. 
Facies in medio cariuata, nec distincto excarata, nec luberculata. Oculi nudi, in fronte maris non late contigui.

Pedes femoribus posticis valdè incrassatis, per totam longitudiuem inferne serrulatis; tibiis posticis satis arcuatis.

Ala venis longitudinalibus quarta et quinta ad apicem coujunetis. satis longe a costali; quinta et sexta in parte exteriori cubitatis, supra cubitum valde sinuosis: transversaria exteriore paulo magis proxima interiori, quam apici lougitudioalis sesto ubi conjungitur quiutie.

Sprcies Italica luica 1. Pipigins Lin.

MAAGSAAOM

Observal. et Synonim.

Sp. 1. S. Pipizns Lin. Fabr. Rossi. Panz. Sto-F.-S: Latr. Fall. Macq. Mgn. Zett. Loëw. Rndn. Walk. Vulgatissimus ut in fere lota Europa, etiam in tota Italia. Parmæ legitur a martio ad novembrem. 
Cenus 10. Ascra Mgrl. Mgn. Latr. Macq. Loëw. Zett. Rndn. Walk.

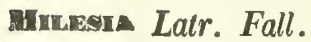 \\ Mimodon Fabr. \\ Srapmes Panz.
}

\title{
Cuaract. Genek.
}

Antenne articulo tertio plus minusve elongato-sub-ovato: arista dorsuali nuda, is medielate basali articuli inserta, el breviore articulo ipso.

Epistominm productum sub-acuminatum: facie nec carinata, nec $t \mathbf{u}$ berculata, obliqua, et in mare paulo concava.

Oculi nudi, in fronte utriusque sexus magis vel miuus distantes.

\$bdomen prope basim eylindrice et distincte coarctatum, præsertim in fominis.

Ale venula transversaria exteriori, magis proxima interiori, quam apici luagitudanalis sextæ: quinta et sexta longitudinalibus, extrinsecus, angulo recto cubitatis, el præcedentibus perpendiculariter conjunetis.

pedes femoribus posticis satis incrassutis, inferne seriatim denticulatis: tibiis posticis arcuatis.

\section{SPEcies Italice:}

A. Abdomen segmentis secundo et tertio rufofusciatis, vel maculatis. 
B. Anterno articulo tertio distincte elongato-orato. Sp. 1. Podagrica Fabr.

P3. Antennœ articulo terlio manifeste breviter ovato.

$$
\text { Sp. 2: Quadripunctata Mgn. }
$$

A. Abdomen vel unicolor, vel segmento secundo tantum rufofasciato, aut maculato.

C. Abdomen segmento secmino rufo-fasciato, vel maculato. Sp. 3. Dispar Mgn.

CC. Abdomen nig̣ ro-unicolor'.

$$
\text { Sp. 4. ENea Mgn. }
$$

Observat: et Synonimin

Sp .1. A. Podagriea Fabr. Fall. Mgn. Maqu. Loëro Zelt Rndn. Wall.

v. Descript, Zetterstedtii.

Species in tota Italia vulgaris; in ag̣ro parmensi uhimi? comunissima, mensibus præsertim main et jr nio. Istius exemplaria miserunt, De Cristofori, Villa, at D'Arco, ab Insubria: Genens et Bellardi, a Pedemontio: Muscino, a Liguria: Bernoloni a Bononia: D. Costa a Regn., neapolitaro. 
102

Sp. 2. A. Quadaipenctata Mgn. Mac. Zell.

v. Descript. Zętterstedui.

Semel individuum fremineum lajus speciei, a me captum fuit, mense junio, in colle sub-apeunino agri parmensis.

Sp. 3. A. Dispar Mgn. Macq. Zett.

v. Descripi. Zetterstedtii

Semel uterque sexus ab Equite Genéo missus, in Pedemontio inventus.

Sp. 4. Aั̃oEnea Mgn. Zelt.

V. Descript. Zellerstedtii.

Duo exemplaria possideo hujus speciei, quorum patria mihi ignota, iden dubitanter eam adscribo dipteris italicis. .

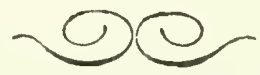

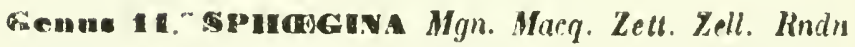
- Walk.

Miresua Fall.

Canaract. Geachich.

Antenna articulo lertio sobcirculasi; Arista dersuali, nuta, propo basim artianli sita. 
Oculi nudi, in fronte utriusque sexus, magis rel ininus distantes. Epistomium distiucte productum, sed non rostif-forme, facie in midio satis excavata.

Abdomen prope basim value, et cylundrice coaretalum.

Pedes femoribus posticis valde incrassatis, subtus scriatim epiuulosig: tibis posticis non distincte incurvatis.

Ala vena quinta longitudinali extrinsecus arcuatim, et non angula. tim nexa; sexta angulo obtuso cubitata, et oblique, non perpendiculariter, priecedeuti conjuucta: transversuria exteriurs magis proxima interiori, quam ap̧ici longitudinalis sezte.

Sipent:s ITAlice

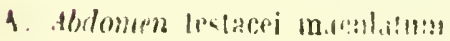

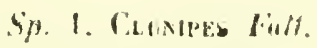

A. Alommen lotuan niğrmm.

5p. 2. Nustes Mirn.

Ubservat. et Synoni:n.

Sp. 1. S. Clunipes Fall. Mgn. Macq. Loëlo. Zell. Rindn. Walk. v. Descript Zettersiedtii.

In apennino parmensi raro inventa a D. Berléo, et una vice tantum a ue, in collibus subupenninis.

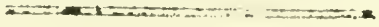


Sp. 2. S. Nigra Mgn. Mucq. Zell.

V. Descript. Zetterstedtii.

Unicum exemplar speciei in collectione mea asservatum, a Pedemontio olim misit Prof. Genètıs: sed habitat quoque in regno Neapolitano, teste Doct. Costo.

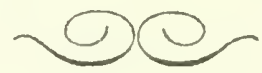

Gesus 12. Hace Fabr. Mgn. Fall. Latr. Macq. Loëw. Zett. Rndn. Walk.

\section{Characteres Generici.}

Antennx articulo tertio subcirculari; arista dorsuali nuda, ad basim articuli inserta.

Oculi nudi, in fronte maris late cohereutes.

Epistomium noo productum; facie tuberealata.

Abdomen iu segmento secundo satis et cylindrice coarctatum.

Pedes graciles, femoribus etiam posticis exilibus.

Alce vena transversa exteriori, magis proxima interiori, quan apici longitudinalis sexta: quarla longiludinali oriente a tertia, contra transversam interiorem. 
Species Italic.e.

A. Abdomen fasciis testaceis duabus.

B. Antennoe testaceæ vel fusco-testaceæ: alce non lotie, eł parum infuscatæ.

Sp. 1. Elongata Fabr.

BB. Antennae nigræ, vel nigricantes.

Alce totæ et distincte infuscatæ.

Sp. 2. ObsGuripennis Migr.

AA. Abdomen segmento secundo tantum, basi testacei fasciato. Sp. 5. Scetellata Mgn.

Observat. et Synonim.

Sp. 1. B. Elongata Fabr. Fall. Mgn. Loëu. Zell. Marace. Curlis. Rndn. Walk.

TaBida Mgn. Macq:

Vide Descript. Zellerstedtii.

Species communis in agri parmensis planitie et collibus, et etiam in apenninis clalioribus non rara, a mense majo ad septembrem legilur.

Sp. 2. B. Obscuripennis Mgn. Zelt. Walk.

Vide Descript: Zetlerstedtii. 
Semel mas speciei, mihi a Pedenontio usissus, ab) Equili: Genèo.

Sp. 3. B. Scutellata MIgn. Macq. Walk.

V. Descriptionem foeminæe in Op. Meigenii.

Marem speciei inveni, una vice tantum, in collibus ditiouis parmensis, qui difert a fremina, segmento quinto abdominis immaculato.

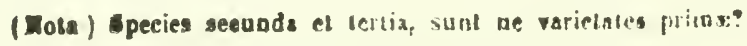

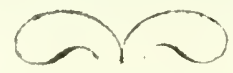

3

Genu* 13. spazicastes: IMAn. $\left(^{*}\right)$.

ormpunoma $A \cdot \cos a(* *)$.

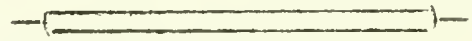

Charpet. Gener.

Antemne articuln tertio subnvalo: arista dorsuali, longn, pilosula, prope basim articuli iuserla.

Facies supra epistomium non productam. Lubercule distincto predita. Oeuli nudi, in frnnte maris late cuherentes.

(1) Magarin de Zoologie 18:8x.

(") C. Ball. Fico 1857. 
Abdomen prope basim distincte coarclato-depressum.

Alce ut in Bacca venosæ, excepta vena quarta longitudiasti, salis interius oriente, ante transversam interiorem.

Pedes non crassi, sed noo satis cxiles, tibiis posticis in feen in minus' in mare satis contortis, et in utroque sezu excaratis magis vel minus.

\section{SPECIes ITALic.}

A. Abdomen rubrum basi et apice nigrum.

\section{Sp. 1. ApenNIX Findn.}

AA. Abdomen omnino nigromescesess.

Sp. 2. Lutiunis A. Costa.

Observal. "t Synonim.

Sp. 1. S. Apennin Rindn (op. e.)

Ambelans? Fabr. 'Mapra? Loüu

Rarissima species in Apennino inventa a Docinte Berléo, eq a me semel in collibus stba peruinis agri jarmensis. $\left({ }^{*}\right)$

() Dom. Locw. credil Sp. meam Milesix Ambulantis Fabriei pertinere, sed hoc nou esse pulo, quia in brevi diagnosi ejusdean auctoris, segmentum tertium abdominis, lateribus macula uigra sigualum dicitur, quil non sonrenil aperiei nustrx. 
Sp. 2. S. Lugubris A. Costa.

Alpinus (olim) Mihi. in Litt.

Similis forma Spaz. Apennini, sod paulo minor et distinctissinus, colore omnino nigro, in Thorac is dorso, et abdominis paulo onescente, et exceptis calipteris albidis, clava halterum lutescente, et pube, albicante lutea in parte infera tarsorum. - Distinctissimus quoque, tibiis posticis valde contortis et excavatis, magis quanı in altera specie.

Semel marem accepi a fratribus Villa mediolanensibus, qui eum legerunt in alpibus insubrix. In Regno' neapolitano nuper lectus a D. Costa.

V. Diario G. B. Vico 1857.

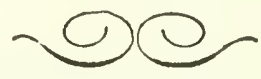

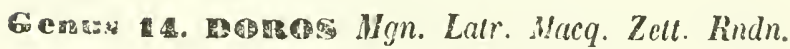

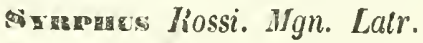

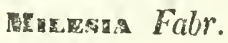

seres Fall.

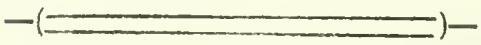

Cuaract. Generici.

Antennce articulo tertio sublenticulari; arista dorsuali, brevi, crassinscula, nuda: prope basim articuli inserta.

Oculi oudi, in fronte maris, prope verticem approximati, sed non contigui, fronte antice lata, in foemina latiore. 
Facies supra epistomium non productum, distincte tuberculata. Abdomen segmento secundo cylindrice valde coarctato.

Pedes non exiles, sed nou incrassati.

Ale circiter ut in Bacca vonosæ, sed excepta reaa longitudinali quarta, satis propius basi alarum oriente, antc veuularn transrersam interiorem.

Species Italuca unica 1. Conopseus Fabr.

Ohervat at Symoni:n.

Sp. 1. D. Conopseus Faur. Rossi Fall. Mgn. Macq. Zelt. Rndn. Walk.

Cosnctatus Punz.

v. Descript. Zetterstedtii.

Rarissimo in collibus et montibus ditionis parmensis, a Doct. Bertéo el a me captus: ejusdem exemplar a pedemontio misit Bellardi, et all Tergestun captus a D. Schiner.

(Nota) Characteres Generis istitis Syrpho festivo Fabricii et Syrpho ornato Meigenii non conveniunt, ideo non conjungendi, ut faciunt non nulli auclores, cum Conopreco in unico genere, sed melius cum antiquo Syrphorum.

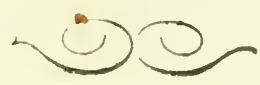


Genas 15. Serhatiophoma St.-F.-S. Haeq. Zett. Rndn.

Sceva Fabr. Fall.

tivepaes Fabr. Rossi Latr. Mgn.

Muera Lin.

MELTHegrew Loëlo. Walk.

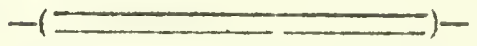

\section{Characteres Generici.}

Antennce articulo tertio brevissime ovato, seu subleuticulari; arist s dorsuali, modice elongata, cxili, nuda, ad basim articuli inserta . Facies iu inedio subcariaato-producta, et tuberculata.

Ocu'i nudi, in fronte maris modice late contigui.

Abdomen prope basim non coarctatum distincte, apice in maribus subtus inflato et penicillato, seu vesica anali, tuberculo iafero, et squama veutrali pilosa instructum.

Alis circiter ut in Bacca venosæ, sed exceptis, veuis prima seeunda et teria lougitudinalibus, in costali sub-æque distantibu s, et vena quarta, distincte ante transversam interiorem, a tertia oriente.

p.t.s simplices: seu non incrassati.

\section{Species rralice}

A. Ala maris, aldomine multo breviores. (")

i) Character's marium in hac tabuta tantum adhibiti, quis famiua

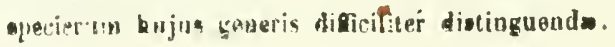


B. Pedes luti flarescentes.

\section{Sp. 1. Sgripta Lin.}

BB. Pedes feurorum basi, el coxis plus minusve nigricantibus. Sp. 2. Nigricoxa Zelt.

AA. Ale non, aut vix in Eutroque sexu, abdomine breviores.

C. Pedes toti flarescentes.

D. Vitte flave laterales tlıoracis, ad radicem alarum non interruptæ. - Abdominis fascia flava basalis, margine postico subrecto.

$$
\text { Sp. 5. Teniata Mgn. }
$$

DD. Ville laterales thoracis, ad radicem alarum interruptæ. - Fascia flava prima abdominis, margine postico incurvo.

Sp. 4. Oleandri Schmbr.

CC. Pedes femoribus basi llus minusve nigricanle.

E. Scutellum totum fllavum.

F. Vitte flavi laterales thoracis, ad radicem alarum non interruptæ.

$$
\text { Sp. s. Piota Mgn. }
$$

FF. Vitte flavæ laterales thoracis, ad radicem alarum interruptæ.

$$
\text { Sp. 6. Menthastri lin. }
$$

EE. Scutcllum fusco nigricans, limbo anguste flavo.

$$
\text { Sp. 7. Sвири.. Mihi. }
$$


Observat. et Synonim.

Sp. 1. S. Scrıpta Iin. Fabr, Mgn. Loëw.Zett. Rrsdn. Walk. Mexrinstri Fall.

V. Descript. zctterstedtii.

Vulgalissimd in tola lialia, et rjusdem exemplaria mihi.

llansmissa finemut a Petemontio, Liguria, Insubria, Elruria, Bononia, Neapoli, Sicilia, et Melita. - In aggro parmensi legitur passim, a mense martio ad oitobrem.

Maris abdomen variat latitudine, forma, et etiam colore fasciolarum dorsualium, ita ut aliquando, istud plus minusve laie ad apicen rufun sit, fasciis nigris obliteratis, vel decoloribus. Tamen in hac specie fasciolas tres anteriores flavidas, non interruptas (tertia tantum sepius, el secunda aliquando, magis vel minus emarginatis) in utroque sexu observavi.

sp. 2. S. Nigricoxa Zell.

v. Descript. Zetterstedtii

Vulgaris ut S. Scripta in tota Italia, iisdem locis et temporibus invenienda, tamen istd magis frequens in mensibus autumnalibus.

Distinctus a mare precedentis, colore coxarum et femorum, magis vel minus nigricante, et uterque se$\mathrm{xus}$ difert, fasciolis flavidis, prima, semper et tertia sepius, nisi omnibus interruptis, vel subinteruptis in medio. 
Sp. 5. Taemiata Mgn. Macq. Loüu. (Zett? Valk?)

V. Descript, Meigenii et Macquartii.

Maris exemplaria in agro parnensi capta characteres præbent S. Tanialce Meigenii, qui cum Macquartio de femorum et coxarum colore silet

In nostris, femora omnino flavescentia, ut coxie, dum Zetterstedtius, coxis fuscis, et Walkerius femoribus hasi nigricante, speciem distinctam esse affirmant.

Sed sı Gallicus et Germanicus Dipterologi, de nigredine femorum et coxarnm non loquntur, aut ista numquam adest in specie typiea, aut in varietatibus tantum ejusdem vix observanda, ideo recte exemplaria nostra Meigenianæ speciei Toniata referenda sunt. - Individua femoribus paulo nigricantibus Walkerii, forte S. Menthastri vel Pictæ pertinent, a quibus tamen differre debent, fasciis lavis abdominis integris, non interruptis in medio.

Fœmina tœniate distincta tantum est a fœmina Scriptœ, statura satis minore.

Sæpius in individus ltalicis masculis, segmenttorum apicalium abdominiis color fulvus dilatatus, et aliquando tan lum, ut vittas nigras prope basim eorum vix ostendat; alibi color niger in ferrugineum vel rufum mutatus.

Non infrequens sed non vulgaris in agro parmensi, ut in Pedemontio et in Insubria, ubi Bellardi et Villa le ${ }^{z}$ gerunt.

Habitat cum præcedentibus, iisdem temporibus et locis. 
Sp. 4. S. Oleandri Schembri (in titteris?.

Quamvis unico exemplare masculo, in Melita invento a Docl. Schembri, species instructa sit, tamen ab aliis salis distinctam esse puto.

A speciebus cum quibus abdominis lirevitalem commonem habet, difert, a Toniata et Picta vittis flavis laterilibus thoracis ad sadicem alarum interruntis: a Spursropuronia Menthastri vero, quæe characteres isios pessidet, diversa est prasertim fascia flava basali alutuminis integerrina, lata, et sic curvata, ut ad latera angulis posticis segnenti proprii innixa sit, ex difert etiam colore pedum omnino flavescentiun ab ista et a picta, quxfeniora plus minusve nigricätia all balsim præelsent.

In specimine meo, abdomen ad apjicem rufum et non nigrofasciatum ul in varietatibus observatur aliartm spercierum, et ideo etiam in hac Sphærephoria cubro iste facile non est nurmalis.

Sp. 5. S. Picta Mgn. Macq. Zell.

V. Deseripl, Zellersledtii.

Huic speciei pertinere credo fominam fasciis abdominalibus omnibus interruptis, et deabus ultimis ad latera conjunctis, sic ut anulos efforment, macula ovala nipged centrali in urroque inclusa.

Speciem hanc raro cum præcedentibus in dilione Parnicusi inteni 
Sp. 6. S. Hextilastri Lin. Falm. liossi. Fall. Mgn. Zctl. Nacq. Walk.

V. Descript Zellerstedtii.

cui adde: Haris frons, vitta transversa incisa supra foveolam intenniferam, aiiquando tamen parum distincta.

Thorax fasciolis favidis lateralibus, prope radicem alarum interuptis.

Non infrequenter, in aldominis segnentis apicalibus, ut observavi in speciebus precetentious, etiam in ista, color ferrugrinens vel rufus, loco fusciarum nigrarmadest. in tiatsctilis.

Fomina speciei mihi videtur ab affinibus diversa, præcipue forma fasciarum abdominis, in duobus ultimis segmenlis lunulas fruatnor flavas subequales constituentium. In arro parmensi non infrequens legritur cum congeneribus. - In Regno neapolitano quoque vivit, captus a D. Custa, et in Etruria reste Rossio habitat.

Sp. 7. S. Serpilu Mihi. Long. Mill. כ.

Alo longitnline circiter abdominis. - Thoracis vittx flavæ luterales ad radicem alarum interrupti. Scutellum, fusco-nigricans, limbo angusto flavo. - P'edes femoribus posticis late, anterioribus quatuor anguste nigricantibus. Abcomen basi nigra, fasciola flava prima in medio interrupta: segmento tertio basi uigrn postice struginon; quarto basi et apice rufo-ferrutuginte, in mentis fuscia fulva; quinto rufo. 
Species colore scutelli distinctissima ab omnibus congeneribus, pro unico specimine masculo instituta Parmæ capto, cujus facilius varietates extant quoad colorem abdominis.

(Nota) Nelle femine di tutte le specie di questo genere, le gambe sono più o meno gialliccie completamente, e le fascie trasversali dell' addome sono assai variabili, per cui la loro distinzione riesce assai difficile, e perciò può dirsi che $e_{\text {sono }}^{\tau}$ i maschi soltanto, sui quali le specie sono fondate.

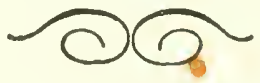

Conuw. 16. perecocera Hofg. Mgn. Zel. Rndn. Rmyseia Fall.

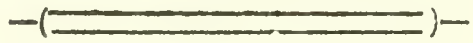

Churact. Gener.

Antenne arista brevissima, et distincte artieulis tribus instructa. Cœters ut in generibus proximis. 
Sprcieg Italica

1. Tricivera Hofg.

\section{Observat. et Synonim.}

Sp. 1. P. Tucincra Hofg. Fall. Mgn. Macq. Rndn.

Species nondum in Italia capta, sed pro certo habeo eam in aliqua nostra provincia habitare, nam in finitimis regionibus Galliæ et Germaniæ lecta fuit.

Exemplar collectionis meæ a D. Macquartio missum, in Gallia meridionali inventum, ideo cum nostratibus speciem hanc collocavi.

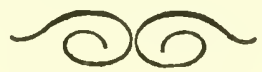

Senus 17 (a) PaATychrinus St.-F.-S. Loew. Srapius Fabr. Mgn. Macq. Rndn. Walk. Scava:Fabr. Fall. Zell.

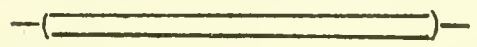

Characteres Gengrici.

Autenna Oculi Facies Alce elc. ut in genere Syrpho, et distinctus ab eodem, Pedibus anticis tantum, tibiis tarsisque præsertim in inare dilatatis; in fomina articulis intermeaiiis longitudine latioribus (1).

(i) Furte cun gencre Lassopurico meo, istud quoque conjuogendune 
Species Italic.ri.

A. Tarsi postici saltem in medin listinte fulwsecusus.

B. Tarsi antici maris hand valde difatati. Tibie aritice in eodem sexu distincte dilatato-compresce. - Urriuscrue sexus, titice.postice antilo nigricante.

$$
\text { Sp. 1. Chypeates Mgn. }
$$

BB. Tarsi antici maris cum libis value dihatati. - Maris et fœminæ, tibire posticie tote fulvescentes.

$$
S p \text {. ¿. Fulviventris Macq. }
$$

A . Tarsi postici omnino nisri.

C. Alce non distincte infuscalx.

D. Aldomen naculas quattor probens, maris lutescente albicantes; fomina coruleo-albidas.

$$
\text { Sp. ̄. Albinanes Fabr. }
$$

et sic integrum Srapur antiqum geaus servandum. quod in tribus seclionibus dividere possumus, nisi in generibus, sic distinetis.

Pedes antici libiis et tarsis salis aut valde in mare dilatalis, in fomina tarsis anticis brevibus, arliculis transversis.

Secrio 1. (Geans Platycumas St.-F.-S.)

aa. Pedes antici, in utroque seru forma ordinaria.

b. Oculi omnino nadi in utroque sexu.

Sectio II. (G. Syrous Fabi.)

at). Oculi in mare magis vel minus pilosi, in focmina aliquando 10mentos: aliquando partam manifeste puberult.

Secrio III. (G. Lessopurucus Rildn.) 
D). Abrlomen maculis saltem sex lutescentibus vel rufescen(i)uss.

E. Pcdes quatuor anteriores, saltem extrinsecus albo-lutescentes.

$$
\text { Sp. 4. Scutatus Mgn. }
$$

ER. Pedes omnes nigri, geniculis magis vel minus late rufescentibus, vel tibiis ad basim.

F. Epistomium satis productum. - Tarsi intermedii nigricantes

Sp. 5. Manicatus Mgr.

Fl. Epistomium parun productum. - Tarsi intormedii albicantes.

Sp. 6. Parmatus Mihi.

C.C. Alo distincle infuscatx.

Sp. 7. Seathulatus Mihi.

Observat. et Symonim.

Sp. A. P. Clypeatus Mlgn. Macq. Zelt. Loëio. Walk. V. Descript. Zetlerstedtii

Maris exemplar semel accepi a fratribus Villa Mediolanensibus, qui eum in alpibus lısubrix legerunt. 
5p. 2. P. Fulviventris Macq. Zell.

v. Descript. Macquartii.

Fœminam speciei non raro legri in planitie et collibus ditionis parmensis, sed marem rarissimo - Fomina mensibus aprile et majo legilur, mas bis tantum mense martio inventus.

Sp. 3. P. Albumanus Fabr. Fall. Mgn. Maeq. Loëro. Zell.

v. Descript. Zetterstedtii.

Species non frequens in agro parmensi, mas rarior. - Legitur uterque sexus minus infrequenter mense septenbre in collibus subapenninis.

Sp. 4. P. Scutatus Mgn. Alacq. Loëzo. Zelt.

v. Descript. Zetterstedtii.

Non rarus in parmensi ditione primo vere, præsertim in locis monluosis. Hujus spec: exemplar at Pedemontio misit Bellardi.

Sp. ว. P. Manigatus Mgn. Macq. Zelt. Walk.

Vide Descript: Zetterstedtii.

Rarus in Apennino parmensi, ubi nonnulla exemplaria utriusque sexus a me lecta fuerunt, mense Julio. 
Sp. 6. P. Pakratus Mihi Longil. ilill. 11.

Similis mari manicati, at distinctissimus.

1. Epistomio manifeste minus producto.

2." Tarsis anticis totis, et non articulis tantum dilatatis, albidis.

3. Tarsis intermediis albicantibus, non nigris.

4. Halteribus clava partim fusca, et non ounino albilu-luteis.

5." Maculis abdominalibus subxque longis et latis.

6. Melalarsis posticis satis uinus incrassatis, quamvis crassis.

A Platicheiro Pillato, cui quoque proximus, satis difert.

1. Aniemnis tolis nigris, non subus rufo-maculatis.

2. Tarsis antiris, articulis duobus uilatatis, et non uno tantum,

$\therefore$ Metaturso intermedio albido, non nigro etc.

Marem micum speciei, in alyibus pedemontanis captum, olim a Genco accepi.

Sp. 7. P. Spatiulatus Mihi. Longit. Mill. 8. Antenne nigræ.

Facies et Frons nigro-œnescentes, lateribus pallide pollinosis.

Thorax ænescens viltis tribus fuscis dorsualibus, intermedia latiori, et distinctiori.

Scutellum œneum pilis nigris et ciliis marginalibas luteis. Ilalleres fusci, stipite pallidiore. Alce distincte fuscr.

Abdomen dorso nigro-opaco, lateribus œenescentibus: segmentis tertio et quarto, maculis lateralibus duabus luteoneneis; quinto maculis lateralibus, et segmento sexto aneis. 
Pecies nigri, geniculis omnibus, libiarum anticarum et intermediarum apice, tarsis anticis totis, el intermediormm basi lutescentibus. - Tarsis anticis nodice dilatatis; tibiis paulo al apicem latis. - Mctatarsis posticis haud incrassalis.

Mas speciei a me bis captus, prope Parmam mense octobre, in floribns Danci Carolae una vice, et altera vice in collubus ditionis ejusdem, mense septembre, in floribus solidaginis.

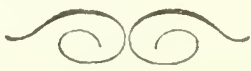

Genas. 7. (b) Sverrites Fabr. Rossi. Latr. Mgn. Macq. Loëw. Walk. Rndn.

Scava Fabr. Fall. Zett. Conoses Scop.

Cinestaes part. Rerenesa part. Fabr. piesca Lin. Petagna.

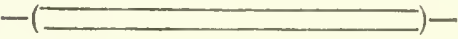

Ciraracteres Generici.

Antenne articulo tertio avato, vel sub-ovato, arista dorsuali, nudil, longiuscula.

Oculi nudi, in fronte magis vel minus late coherentes.

Facies supra epistomium tuberculara.

Ablomen prope basim non manifeste coarctatum. 


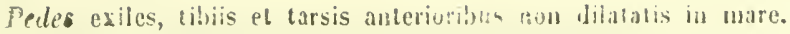

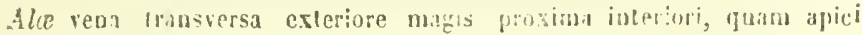

longitudinalis sexı trihus prinus in costali varie distantibus, numquam oque di. stantibus: spuria supranumerum ordmaria, ubraque satis distucta, nee un unedio nec prope basia internptia.

Sirces IT:Hot

A. Mrare muicelures, sall nom dislione havomaculatæ.

B. Scatelium nigrum, magis vel unms virescens vel coerulescens, sed nou! branslucidum.

a. Julomen nigrum macolur.

1. Unicolon Mili.

aa. Ablomen fluvi vol lutei faccialum vel macu'alum. C. Abdomen segumento unico, llavo-fascialo vel maculato. Sp. ¿ Rossnua Fabr.

Cr. Ablomen flavi saltem bifusciatum, vel segurntis saltem - duobus bimacnlalis.

D. Tarsi omnes et toli nigri

Sp. 万. IIvalivates Fall.

DD. Tarsi aliqui, sapins internedii, saltem basi magis vel minus late rufescentes.

E. Antenne arliculis duobus primis nigricantibus.

f. Femora postica tola, ant maxima parte fulva; libiis pa- 
riter fulvis, rel anulo fusco tantum manifesto, et angusto.

\section{Sp. 4. Scalaris Fabr.}

Fr. Pedes postici, magis vel minus late nigricantes, femoribus fere totis, et tibiis late nigrofuscis.

$$
\text { Sp. 5. Mecunus Lin. }
$$

EE. Antenna articulis primis lutescentibus.

$$
\text { Sp. 6. Gracilis Mgn. }
$$

BB. Scuellum flavum vel Davescens, et si fusco lutescens tunc translucidum.

G. Pedes postici, tibiis et femoribus fere totis nigricantibus, vel late nigro-anulatis, et tunc abdomen faseiis luteis in maculas divisis.

II. Tibice quatuor anteriores, ut posticæ fere totæ, nigricantes. - Abdomen segmento quarto postice haud lutei marginato.

\section{Sp. 7. Nigritiblus Mihi.}

HH. Tibice quatuor anteriores lutescentes, anulo fusco vel parum manifesto, vel satis angusto. - Abdomen segmento quarto, margine postico saltem in medio lutescente.

I. Macula abdominis latiusculæ, obliquæ, et saltem intermediæ, margini segmentorum præcedentium subøonเig!n.

Sp. 8. Maculiconsis Zelt. 
II. Maculœ abdominis angusłæ, subparallelæ, et distantes a margine segmentorum præcedentium.

\section{Sp. 9. Unbellatarem Fabr.}

GG. Pedes postici vel toti lutescentes, vel tantum femora, apice excepto, nigra; ant raro anulis tibiarum vel femorum nigricantibus, et tunc abdomen fasciis flavis in maculas non divisis.

K. Abdomen maculis a margine postico segmentorum præcedentium, æque et manifeste distantibus, forma subquadratis.

\section{Sp. 10. Decores Mgn.}

KK. Abdomen vel fasciis luteis in maculas non divisis, vel maculis lunulatis aut transversis margini segmentorum præcedentium, saltem intermediorum, contiguis. L. Frons tota ut vertex luteo pilosa.

$$
\text { Sp. 11. Cinctus Mgn. }
$$

LL. Frons, aut vertex, aut una et alter, pilis nigris vel nigricantibus.

M. Pedes postici tibiis et femoribus, nigro vel fusco-anulatis.

$$
\text { Sp. 12. Aunicolus Mgn. }
$$

MM. Pedes postici, tibiis haud nigricante anulatis; femoribus vel tolis fulvis, vel basi fusca vel nigricante, plus minusve lata.

N. Abdomen fasciis luteis in aliquo segmento duplicatis. Sp. 13. Baltueatus DeGer.

NN. Abdomen fasciis luteis in nemine segmento duplicalis. 
O. Abdomen semninto ruarto postice, haul lutei marginato, uninio rolo aigro.

\section{Sp. 14. Bifasciatus Fabr.}

ก․ Ablomen ergmento qurarln poslice lulei marginalo; quin'o maculas luteas præbente, alıpuando parvas.

P. Faries in merlio han! nigro-viltata, arl summum narum et anguste nigricans sub tuberculum faciale. - $\boldsymbol{A} b$ domm fasciis in!ermeniis interruntis.

Q. Pedes tarsis femoribusque omnibus, in utroque sexu lutescentibus omnino, vel fulvis.

\section{Sp. 15. Nitidicolls Mgn.}

QQ. Pedes, tarsis mosticis nigris vel nigricantibus, ut femorum hasis, presertim in maribus.

R. Frons maris anlice fulva poslice nigricante: formine in parte anlica fulva, macula subtrigona nigricante.

S. Femora in maribus lantum hasi late nigricantia. - sbdomen fasciis lateis in utroque sexi parum postice cmarginalis.

Sp. 16. Rißesu lin.

SS Femora in utroque sexu basi nigricantia. - Ahdomen in utrogue sexu fasciis luteis poslice value emarginatis.

Sp. 17. Tiramenvis Mgn.

RR. Frons maris tota fulvescens: Fourinx antice, macula nisricante destilula.

Sp. 18. Fuvicigs Bihi.

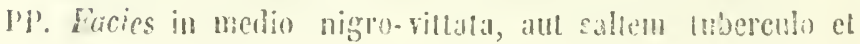


parte postica nigricantihus, et si raro tuberculo non nigro. tunc fascix luter abdominis in medio magis vel minus distincte interuptæ, ant fere interruptr.

T. Peristomium margine 10to, late nigricante. - Femora postica latissime nigricantia.

$$
\text { sp. 19. Buccllatus Mili. }
$$

TT. Peristomizm hanl totum nigro-marginatum. - Femora postica aut non, ant ima basi tantum nigricantia.

U. Abdomen maris, maculis segmenti tertii margine postico recto. - Femora guntuor anteriora basi, in fominis, late uigra. - Antenna in utroque sexu nigrat val nigricantes.

$$
\text { Sp. SO. Conolue Fabr. }
$$

UU. Abdomen maris, macuis segmenti tertii postice rolundatis. - Feinora omula, in feminis vel non, rel ima basi tantum nigricantia. - Antcnno in utroque sexu rufescentes, saltem inforne.

Sp. 21. Luniger Mgn.

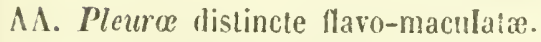

V. Pedes toli et omnes fulvi vel lutei.

Sp. 22. Festivus Fabr.

IV. Pedes postici apice femorum el libiis, vel nigrricantibus, vel ferıugineis.

X. Pleuroe macula unica flava disincla. 
XX. Pleur $\alpha$ maculis qualuor flavis distinctis.

\section{Sp. 4. Dives Miki.}

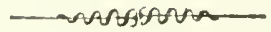

Obsercal. et Synonim.

Sp. I. Unicolon Mihi. Longit Mill. 6 (Farm.)

Totus nigin-nenescens, exceptis antennis inferne fulvescentibus: culipteris albis, Halteribus luteo albicantibus: pedibus luteis, apice tarsorum et annulo tibiarum poslicarum nigricanlibus. - Alis griseis vitta marginali Iutescente, Fucie et Fronte prope oculos paulo albidi pollinosis.

Fæmina speciei a me semel lecla in apennino parmensi, et semel prope Florentiam a D. Piccioli.

Sp. 2. S. Rosanui Fubr. Mgn. Macq. Loëw. Zell. Walk. Nuctilegus Panz.

V. Descript. Zetterstedtii.

Species rarissimn capta a Doct. Bertèo in apennino, et in collibus ditionis parmensis a me semel inventa.

Sp. 5. S. Hyalinates Fall. Mgn. Macq. Zett. Walk. V. Descript. Zctterstedtii.

Non rarus in collibus subapenninis agri parmensis, alibi rarus: Fœuna minus frequens. - A mense junic 
usque ad octobrem enm inveni. - Exemplar fœmineum in insula Melita captum a D. Schembri accepi.

Sp. 4. 5. Scalaris Fabr. Latr. Panz. Mgn. Macq. Zell. Walk.

V. Descript. Zetterstedtii.

Species vulgaris in tota ltalia tam in planitie quam in montmosis: in agro parmensi legittr, a mense aprile usque ad octobrem.

Sp. अ. S. Melunus Lin. Fabr. Rossi. Fall. Latr. Mgn. Macq: Zett. Walk.

Mellarius Mgn.

Meluiturgus Mgn.

IRIS Mgn.

v. Descri pt. Zetterstedtii.

Fræquens cum Scalari legitur, iisdem temporibus et locis; mas vero minus vulgaris.

Sp. 6. S. Gracluls Mgn. Macq.

v. Descript. Meigenii.

A præcedentibus præsertim distinctus, antennis etiam articulis primis lutescentibus, t maris abdomine magis elongato, maculis haud sub-æque longis et latis, sed Inagis elongatis. - Salis diversa a Scalari et Mellina et ab earum varietatibus, nec cum iisdem confundenda, It Clar Zetterstedtius dubitare videtur: 
Species cum præcedentibus, in toto agro parmensi legitur sed minus comunis; ejusdem exemplar a D. Bellardi accepi, in Pedemontio inventum.

Sp. 7. S. Nigritibius Mihi. lengit. Mill. 9-10.

Antennce cum arista nigræ, articulo tertio brevissime ovato, fere subcirculari, inferne aliquando macula parva, rufescente, notato.

Facies et frons nigres, albido-pollinosæ, tuberculo faciali, et apice frontis denudatis, levibus. - Frons ut vertex nigro-pilosa.

Thorax dorso nigroœnescente, et nigro-piloso, pleuris paulo cinerascentibus, albido-pilosis.

Scutellum fusco-testaceum, translucidum, pilis niglicantibus, longis, hirtum.

Abdomen segmento primo, et lateribus secundi, late œuescentibus; secundo in medio, et margini postico nigro, vestigiis macularum testacearum aliquando apparentibus, lateribus ut in sæquentibus albo-pilosis: segmentis tertio quarto et quinto, maculis duabus testaceo-luteis, lateralibus, in primis duobus subquadratis, in ultimo subtrignnis.

Halteres fusco lutescentes. - Calliptera albicantia.

Pedes nigri, geniculis tantum raro late rufescentibus, tarsis posticis, pube infera fulra.

Atœ fuscescentes, vitta stygmaticali parum fusciore.

(Fœm.) a mare difert, pedibus præsertim anticis minus late, et minus intense nigris: et maculis abdoninalibus in secundo segmento magis distinctis, et in cœteris omnibus subtrigonis. 
Species proxima Maculicormi Zetterstedtii, tamen satis diversa, et distincta, præsertim margine postico segmenti quarti abdominalis haud lutei limbato; et pedibus nigrioribus. Non freqienter eam legi in collibus agri parmensi, a mense martio ad augustum.

Sp. 8. S. Maculiconnis Zetl.

V. Descripl. ejusdem $\Lambda$ uct.

Non rarus in collibus et planitic ditionis parmensis, a mense martio ad septembrem.

Non infrequens etiam legitur in Italia meridionali, nam ejusdem specimina accepi a D. Costa in Regno neapolitano, ed a D. Schembri in insula Melita capta.

Sp. 9. S. Unbellatarun Fabr. Fall. Mggn. Macq. Loëio. Zelt. Wialk.

V. Descript. Zetterstedtii.

Species rara, in agro parmensi a me capta, mensibus septembre et octobre.

Sp. 10. S. Deconus Mgn. Mlacq. Zell. Walk.

Vide Descripl: Zetlerstedtii.

Bis tantum syrphum istum inveni, in planitie ditionis parmensis.

Sp. 11. S. Cinctus Fall. Mgn. Macq. Zell. Walk.

v. Descript. Zetterstedtu.

Mensibus martio et aprile raro legitur, in collibus et planitie 
agri parmensis: semel in apennino a Doctore Bertén captus.

Sp. 12. S. Auricolus Mgn. Macq. Zett. Walk.

v. Descript. Zetterstedtii.

Non frequens; præsertim legitur in collibus subapenninis agri parmensis, a mense aprile ad junium; in Etruria quoque inventus, a D. Piccioli.

Sp. 13. S. Baltheatus De Geer. Mgn. Macq. Loëw. Zell. Negtareus Fabr. Panz.

Alternatus Rossi.

Canabinus Scopoli. Pelagna.

v. Descript, Zetlerstedtii.

Comunissimus in tota ltalia, ab Alpibus ad insulam Melitam. In parmensi ditione legitur passim, a primo vere ad decembrem.

Variat maxime latitudine et numero fasciarum abdominaliun. Eius larvæ vivunt ex aphidibus, ut egu obserravi non raro. (Non in ligno putrescente, ut ait Dufour). Vide observat: meas, in diariis. Annali di Bologna 1848.

Sp. 14. S. Bifasciatus Fabr. Fall. Mign. Macq. Zell. Walk. v. Descript. Zetterstedtii

In parmensi ditione non infrequens, a mense aprile ad julium. Prope Tergestum quoque captus a Doct. Schiner.

Sp. 15. S. Nitidicolus Mgn. Macq. Zelt.

v. Descript Zetterstedtii. 
Non rarus in agro parmensi, tam in planitie quam in montuosis, præsertim mensibus Majo et Junio.

sp. 16. S. Ribesu Lin. Fabr. Rossi. Fall. Mgn. Macq. Zett. Rndn. Walk.

V. Descript. Zetterstedtii.

In ditione parmensi vulgaris a martio ad octobrem, ab apennino usque ad planitiem padanam. Hujus exemplaria a Pedemontio, Insubria, Etruria, Sicilia, etc. accepi.

Sp. 17. S. Vitnipenkis Mgrl. Mgn. Macq. Loëw. Zett. Walk. v. Descript. Zatterstedtii.

Vulgaris ut præcedens in tota Italia, et in lociş et temporibus iisdım, in agro parmensi legendus.

Sp. 48. S. Flavicers Mihi. Longit. Mill. 9 sirciter.

Caput facie et fronte omnino flavescentibus; facie luteo-pilosula, fronte pilis nigricantibus, vertice nigro, nigropiloso: epistomio vix sub tuberculo faciali limbo nigricante.

Antennce rufescentes, superne obscuriores, arista nigricante. Thorax nigro-neneus, fulvo pilosus.

Scutellum fusco-rinfescens, translucidum, fulvo-pilosum.

Calipiers ut halteres pallide lutescentia.

Abdomen depressum, nigrum, latelutei fasciatum et maculatum, lateribus arl basim pilis longis albidis, postice breviter nigro pilosis: segmenti secundi maculis dua- 
bus subovatis, marginibus lateralibus productis; segmentorum tertii et quartii, fasciis transversis luteis, postice profunde emarginatis, et margini laterali non produclis. Segmento quarlo limbo postico in medio lutei marginato: quinto fere tolo luteo, vix macula intermedia basali nigra.

Pedes rufescentes, tarsis fuscis, et basi femorum date nigricante, in posterioribus latius, sed ima radice rufescente.

(Fœm)a mare difert, fasciis et maculis abiominalibus latioribus, et postice minus profunde emarginatis. - Pedilus totis fulvescentibus, tarsis tantum fuscis, sxpius in in solo apice. - Fronte antice late et deteminate flavescente, postice cum vertice nigro-œnea, tota breviter nigro-pilosa, summo apice prope autennus nuda.

Raro Sp. hanc inveni, in collibus subapenninis agri parmensis, tempore verno.

Sp. 19. S. Bucculates Milii Longit. Mill. 10. (Fom).

Similis foem: Syrphi Lunigeri, at distincta præsertin ore toto late nigro-marginato, et femoribus posticis latissime nigricantibus: præterea.

Antennce cum arista rufæ. - Frons antice flava, postice cum vertice nigra, maculis duabus lateralibus albidopollinosis. - Caliptera alba. - Ilalteres flavescentes.

- Abdomen Pasciis luteis, segnentorum tertii et çuarti in medio sub-interruptis. - Ventre maculis nigris, transversis, subquadiatis prædito.

redes rufescentes, femoribus omnibus basi late nigricaute, posticis latius. 
Qiramvis fominætantum characteribus innixa sit species ista, tamen satis eam distinctam esse credo, etiam ab affinibus syrphis Lunigero et Corollo, præsertim characteribus oris et pedum.

Exempiar typicum a me lectum in planitie agri parmensis, et olim cum varietatibus Syrp. Lunigeri confusum.

Sp. 20. S. Corolle Fabr. Mgn. Macq. Loëw. Zett. Walk. v. Descript. Zetterstedtii.

Vulgatissimus ubique in agro parmensi, et communis in tota Italia, ab Alpibus usque ad insulam Melitam. Parmæ legitur a martio ad novembrem.

Sp. 21. S. Luniger Mgn. Macq. Zett. Walk.

v. Descript. Zetterstedtii.

Communissimus in Italia, a Pedemontio ad Siciliam. Parmz legitur, a mense martio ad octobrem passim, tam in planitie quam in montuosis.

Sp. 22. S. Festivus Lin. Fabr. Rossi. Latr. Fall. Mgn. Macq.

Citrofaseiatus De Geer. Walk.

v. Descript. Zelterstedtii.

Non frequens in ditione parmensi legitur, minus raro in collibus sub-apenninis tempore autumnali, rarius in montibus elatioribus a Doct. Bertèo et a me captus: 
In Etruria vivit quoque teste Rossio, ct ad Tergestum captus a Schinero.

Sp. 23. S Ornates Mign. Macq. Zett. Wallo.

v. Descript. Zetlerstedtii.

Rarus in ditione parmensi, ubi marem bis tanlum inveni: sed ut videtur fræquens in Pedemontio, nam lmijus specimina in hisce provinciis italicis capta, Geneus, Truqui, Mussino et Bellardi miserunt, sed ommes individua mascula, ideo fomina mili ignota. - Eliam in regno neapolitano vivit teste Doct. Costa, et ad Tergestum teste Schinero.

\section{Sp. 24. S. Dives Miti.}

Similis Syrphis Festivo et Ornato, sed a primo difert, pedum posticorum femoribus apice, et tibiis, tarsisque totis nigricantibus vel fuscis, nec non maculis abdoninalibus latioribus, $\mathrm{ut}$ in ornato, ab isto vero distinctus scutello ad basim in medio non determinate atro, sel exstrinsecus fuscescente ut in festivo: ab illo et isto vero præcipue diver'sus, thoracis lateribus maculis quatuor llavis plus minusve latis et inanifestis: una subovata, perpendiculari fasciolæ laterali flavæ thoracis, inter radicen alarum et caput: altera subrotunda in regione pectoris supera locata, stb perpendicularem, cui fere contigua, vix linea nigra eas sejungente: tertia minori, rotunda, prope originem coxarum anticarum sita: ultima in metalhorace inter alas et halteres observauda. 
Species non rara in agri parmensis planitie et locis montuosis, ubi legitur sepius mensibus majo et junio, sed rarius etian mensibus sequentibus usque al septembrem. - Etian in Etruria et in Pedemontio vivit, lecta a Pecchioli et Bellardi ${ }^{*}$ ).

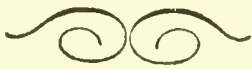

Genus 18. H.STOPHARICES Rndn.

Sxmanus Fabr. Rossi. Latr. Mgn. Mlu'q. Walk. Curtis.

Scoeva Fabr. Fall. Zetl.

Mesca Lin.

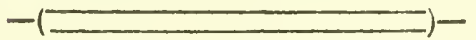

Cimaracteres Generici.

Generis Syrphi Meigenii et Platycheiri Servilliei characterc, prebel, sed ab isto distiuctus tibiis et tarsis anticis non dilatatis, ab omnibus oculis magis vel minus distincte pilosis vel tomentosis.

(i) Adde specicbus Italicis S. Diaphanus Walb. Zelt. cujus descriptionem lege in opere Zetlerstedtii.

Species mihi ignota, sed prope Tergeslum lecta a D. Schiner. 
A. Alce haud nigro-maculatæ. - Pedes maxima parte lutescentes.

B. Pleuree late et distiucte flavo-maculatæ.

Sp. 1. Novus Mihi.

BB. Pleurce haud distincte flavo-maculatx.

C. Fascice abdominales segmentorum tertii et quarti in medio non interruptæ.

D. Facies non nigro-villata. - Abdomen fasciis tertii et quarlı segmenti non profunde emarginatis.

Sp. 2. Topiaries $\mathbf{I I g n}$.

DD. Facies nigro-villata. - Abdomen fasciis tertii et quarti segmenti postice valde emarginatis.

Sp. 3. Albostriatus Fall.

C.. Abdomen fasciis omnibus in medio interruplis.

E. Facies in medio nigro-vittata. - Abdomen basi haud late albila.

F. Líucuice abdominales in segmentis tertio et quarto, in medio et ad latera subæque distantes a marginibus segmentorum precedentium.

G. Frons maris superne non inflata, et oculis non elatior.

$$
\text { Sp. 4. Dignotus Mili. }
$$

GG. Frons maris superne inflata, el oculis manifeste clatior. Sp. 5. Seleniticus MIgn.

lif. Macul a abdominales in segmentis tertio et quarto, in 
modio magis proximx, et ad latera mağis distantes a margine segmentorum præcedentium.

II. Macule quatur intermediæe abdominis latæ, subquadratæ, semper flavi.

\section{Sp. 6. Gemellaril Rndn.}

III. Macula quatuor abrominales intermedix, intrinsecus exiliores, sæyins albicantes vel pallidissime flavescrntes.

$$
\text { Sp. 7. Pүrastri Lin. }
$$

EE. Facirs in mellio haud nigro vittata. - Abdomen basi late a!bicans.

1. Pedes tarsis posticis tolis fulvescentibus.

K. Abdomen fasciis tribus interruptis flavidis. - Frons foem: flava, vilta intermedia nigricinte.

Sp. 8. Glaucus Fabr.

KK. Abdomen fasciis duabus tantum flavicantibus. - Irons form: nigra, apice flavo-marginato.

$$
\text { Sp. 9. Coronatus Mihi. }
$$

II. Pedes tarsis posticis totis niggricantibus.

$$
\text { Sp. 10. Bellandi Mihi. }
$$

A. Ale macula magna nigra notate. - Peles maxima parte nigricantes.

$$
\text { Sp. 11. Lucoruy Lin. }
$$


Observat. el Synonim.

Sp. 1. L. Novus Mihi. Longit. Mill. 9-10.

Antemice cum arista fusco-rufescentes, articulo tertio brevissime ovato, subrotundato.

Caput facie el fonte flavis immaculatis; facie albo-pilosa; fronte pilis fuscis longiusculis; vertice in parte anteriori fusco-piloso, postice pilis luteis.

Thorax niger luteo-pilosus, lateribus vitta longitudinali lata flava: pleuris maculis quatuor flavis sat latis et distinclis, una perpendiculari vitta flavæ laterali llıoracis, altera sub istam sita. tertia prope coxas anlicas, ulima inter radicem alarum et halteres, in melathorace observanda.

Sculellum basi fusco-rufescente, limbo flavo, punctis nigricantibus lateralibus ad basim distinctis, pilis luteis præditun. - Calipteris albicantibus: - halteribus fulvis vel flavis.

Abdomen nigru!m. segmento secundo maculis duabus Muris, sub-ovatis, lateralibus: tertio el quarto, fascia l:ansversa basali, integra, vix in medio postice emarginata: quinto maculis duabus lateialibus, basalibus, subtrigonis: quarto et quinto limbo postico in medio flavo-marginatis.

Pedes omnino lutescentes vel fulvescentes. Ale sublimpidæ, vitta stygmaticali fusco-lutea.

(Frm.) Frons, linea nigra intermedia a vertice, fere usque al apicen sæpius producta. -. Pleura maculis salis latioribus: cæ!era ut in mare.

Non raro speciem hanc legi in collibus, el aliquando eiian 
in apennino ditionis parmensis, a mense majo ad septembrem.

Sp. 2. L. Topianuts Mgn. Zett. Walk.

v. Descripl. Zellersteálii.

Satis rarus in agro parmensi, ejusdem mares duo tantum inveni mense octobre in collibus subapenninis.

Sp. 3. L. Albostriatus Fall. Mlgn. Macq. Loëw. Zell. v. Descripl. Zetterstedtii.

Non infrequens in planitie el collihus ditionis parmensis. legitur a mense aprile usque ad nctobrem.

Sp. 4. L. Dignotus Mihi.

Similis habitu I. Selenilico, cujus characteres fere omnes præbet, sed optime distinctus, fronte non inflata, et oculis non elatiore.

Præeterea paulo minor, minus pilosus, et pilis pallidioribus instructum.

Pro maribus tantum paueis, in agro parmensi a me lectis, species instructa, nam fomina mihi iguota; dubito tamen cum fueminis speciei sequentis eam confusam esse.

Sp. 5. L. Seleniticus Mgn. Loëro. Zelt.

V. Descripl. Zellerstediii. 
Non infrequens in agri parmensis planitie et collibus, primo vere el tempore autumnali; focmina magis communis.

Sp. 6. L. Pyrastri Lin. Fabr. liossi. Fall. Mgn. Maeq. Loëw. Zett. Iindn. Walk.

v. Descript. Zellerstedtii.

Conunissimus ubique in ditione parmensi ut in tola Italia, a primo vere usque ad norembrem.

In stalu larra vivit inter aphides, quos vorat; ejusdem mores primus Vallisnierius observavit.

Sp. 7. L. Grmellarn Rndn. Schin.

v. Descript, meam in diariis.

Annali dei naturalisti di Napoli 1839.

Pro specimine masculo tantum in Sicilia capto, et mihi transmisso a Prof. Gemellaro, species olim instituta fuit, sell nunc excmplaribus nonnullis a D Schembri acceplis in insula Melita inventis, utrogine sexu pertinentibus, speciei distinctio confirmata est, et nuper Parma inventa a me, ad Tergestum a Schinero, et etiam in Germania et usque in Syriam a D. Gödel.

Focmina, exceptis characteribus scxualibus, a mare parum difert.

Sp. 8. L. Graveies Fabr. Fall. Mgn. Macq. ZeW. v. Descrigl. Zetterstedtii.

Spec. rarissimo in Pedemontio lecta a Pr. Bellardi. 
Sp. 9. L. Coronatus Mihi Longit. Mill. 16.

Intennœ atrx, arista paulo rufescente. - Facies flava, in illeso, pallidissime pilosa, cpistomio lateribus inferne late, et limbo supero anguste nigris. - Frons atra levis, submetallica, pallide flavi pilosa, summo apice fasciola flava Iransversa, in medio excavata, prope oculos villa semiovata, albidi pollinosa, all unumequodque latus. - Thorax dorso fuscn-œnescente, pallide flavi piloso, lateribus et pleuris pilis albidis. - Scutellun flavescens, subtranslucidum. Caliptera alba, linea marginali ciliisque flavicantibus.

- Halteres pallide flavi. - Abdomen nigrum albidi pilosum, sfgmento secundo maculis duahus semiovalis, tertio villis duabus transversis, illis et islis a lateribus abrominis remolis. - Alo suldecolores, villa stygmatica lutescente. - Pedes fulvo-flavi, coxis omnibus, femoribus posticis in medio late, anlicis prope basim, anuloque tibiarum, posticarum niģricantibus.

Rarissimo, in collibus ditionis parmensis, speciei feminam tantum legi, mense martio, in floribus Buxi et Grossularix.

Sp. 10. L. Bellardu Mihi.

Similis Glauco fabriciano, at minus angustus, pilosior of aliis characteribus frontis, anteunarum, oculorum ef pedum distinctissimus.

Antermo cum arista nị̧re. 
Facies albido-pollinosa et pilosa, luberculo denudato luteo. Frons fulvescente-pollinosa et pilosa, vertice antice pilis nigris, postice fulvis.

Oculi ad lentem ralidam tantum puberuli.

Thorax niger, dorso rufo-piloso, lateribus pilis fulvescentibus.

Scutellum fulvum, in medio fulvo-pilosum, limbo pilis pallidioribus.

Caliptera rufescentia. - Haltercs lutei.

Abdomen nigrum pilis albidis, apice, et lateribus prope basim instructum; in medio dorsi nigro-pilosum: segmento secundo fascia basali albida, lata, sub integra: tertio et quarto, vittis duabus transrersis angustis, lateralihus, albicantibus.

Pedes fulvescentes, femoribus basi nigricante, posterioribus nigredine magis extensa: tarsis posticis totis nigricantibus.

(Fœm.) Frons hand nigrovittata, sed prope antennas macula angusta nigra distincta.

Abdomen fere totum, eliam in dorso, albo-pilosum.

Pedes femoribus vix ima basi nigricantibus; cœtera ut in mare.

Ex Pedemontio fœeminam a D. Mussino accepi, marem a D. Bellardi.

Sp. 11. L. Luconun Lin. Fabr. Fall. Mgn. Macq. Curlis.

v. Descripl, Meigenii. 
fNon rarissimo in Pedemontio lectus a Truqui et Bell. rdi Vartit basi abdominis lateribus tantum anguste albidis.

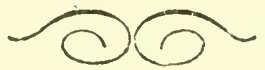

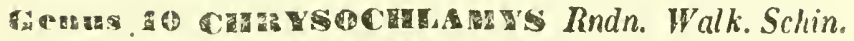
Siarnes Fabr. Rossi. Mgn.

Fimreteris Fall. Zell. Cragerosga Miacq.

Covors Scopoli.

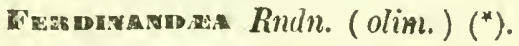

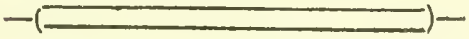

Cuaracterges Generici.

Antenne articulo tertio circulari, lato, arista nuda, in medio dorsi articuli inserta.

Frons distincte producta.

Facies supra epistomium porrectum gibbosa.

Oculi birti, in fronte maris anguste cobcrentes, foemioæ prope verticem parum distantes.

Scutellum setis crassiusculis et longis marginalibus.

Ale vena transversa exteriori fere cequidistante ab interiori $\mathfrak{e}$ ab apice sestæ longitudinalis ubi conjungitur quinı: quarta longitudiaali estrinsecus parum sinuosa, et vix ante transpersam interiorem a tertia oriente: spuria ordinaria ubique distiocta, et non interrupta.

(") Nomen genericum l o", cum Clrysochlamyde mutatum, quia ab tintomologis rejectum. 
Pedes validiuseuli sed non incrassali.

Abdomen prope basim non coarctatum.

Spegies Italice.

A. Facies et genœ haud nigro-viltatx.

B. Frons tota fulva, prope antennas rufescens. Femora omnino flavescentia. - Tibice nullæ fusco anulatæ.

Sp. 1. Cuprea Scopoli.

BB. Frons maris postice nigricans; fœminæ saltenı prope antennas, nigredide magis vel minus lata, lincta. Pedes femoribus saltem anticis et intermediis, basi plus minusve late nigricantibus: tibiis saltem auterioribus, anulo fusco, nunc satis nuc parum dirincto.

$$
\text { Sp. 2. Ruficonsis Falr. }
$$

AA. Facies et genœ viltis nigris distinctissimis tribus.

$$
\text { Sp. 5. Aurea Rndn. }
$$

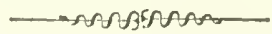

Observat. ct Synonim.

Sp 1. C. Ceprea Scop. Rndn. (Annali di Bologna 18\%1.) Rurıconss Rossi. (Colera Sinonm: Uubia) V. Descriptiones Scopolii et Rosii. 
Facies at frons in hac sprcie lotat fultescentes: pedes omnes el toti fulvi, nec hasi femorum distincte, nee antio tibiarmm manifugto nigricantilius.

Non rara in agro Parmensi, presentim in collibus subapenninis, a mense majo ad angustlm. Legitur quogue in Pedmontio, ubi inventa frit a Bellardi et Mussino: et in Etruria etiam habial Ircta a D. Piccioli

Sp. 2. C. herigonas Fabr. Mun. Hacq. Zell.

Coctera Synon. diutiat.

v. Descripl. Zelierstedtii.

Olin cun varietatibus Crysochlamyris Cuprea ista a me confusa, seủ nunc exemplaria nonnulla observata, ex Danta a D. Sloger, ex Gallia Boreali a Prof. Macfiturt, ex Gernania a D. De-Roser missa, cognovi speciem Fabricianan Ruficornem, a Caprea Scopolis distinclain esse, et revera.

fons maris semper nigricans, antice tamtum anguste rufescen-; formini prope antemas, ef aliquando etiam in andio nigricans, et Pedes in ntroque sexu femoribus presertin anticis et intermediis, basi phus mintisve Jate ot distincte nigricantihus; tibisque annulo fusco, quanvis interdum parum prespicuo, specien fuficurnem a Cuprea recte sejungunt.

lu agro Parmensi et in Pedenonlio legitur non raro cum præcedente, iisden locis et cemporibus. 
Sp. 3. C. Aurea Rndn.

V. Descript. meam in diariis. (Nuovi $\Lambda$ nnali di Bologna 18: i.)

Species facillime distinguenda a congeneribus. vitis tribus facialibus nigris, semper salis perspicuis.

Frequens in collibus subapenninis ditionis parmonsis, presertim tempore autumnali.

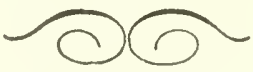

Genus 20 CnELLOSIA Mgrl. Macq. Loëty. Rndn. Míalk. Sxerpers Rossi. Mfgn. Latr.

IGnistaris Fabr. Fall. Zelt.

Musca Lin.

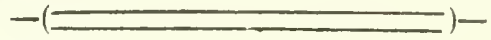

Charact. Gener.

Antemne articulo tertio circulari vel subcirculari, arista dorsuali prope basim articuli inserta, pilosula, pohescente, vel subnuda.

Frons non distincte producta, in freminis lata, sed nec traneversim sulciolata, nec seriatim pusctulata.

Oouli vel pilosi vel pubescentes vel subnudi, in fronte maris plus minusve late coherentcs.

Ala veua quarta longitudinali recta: transversaria exteriore magis proxima interiori, quam apici sextæ longitudinalis: Spuria or dinaria vel ubique parum perspicua, vel prope basin interapta, vel sub indistincta.

Pedes non incrassati. 
Spegies Italic.e.

A. Oexti distincte pilosi saltem in maribus.

b. Ala in medio macula fusca notatæ.

\section{Sp. 1. CEstracea Lin.}

BB. Alce macula nulla distincta.

C. Abdomen ad apicem pilis nigris, alibi ferrugineis hirtum (in fom:) saltem inferne nigro pilosum ad apicem (in mare). Sp. 2. Grossa Fall.

CC. Abdomen pilis vel pube fulvescentibus vel lutescentibus unicoloribus, etiam ad apicem, in utroque sexu.

D. Tarsi omnes, etiam postici, omnino lutei.

Sp. 5. Aurata Fabr.

ID. Tarsi omnes saliem apice nigricantes.

1.. Femora fulvescentia.

Sp. 4. Flavipes Panz.

LE. Femora tota nigra vel latissime nigricantia.

li. Tibice ounes umnino lutescentes.

\section{Sp. 5. Flaviconxis Fabr.}

Hi. Tibice vel totæ nigricantes, vel anulo nigro vel fuseo, lato, et distinclo, saltem in posticis.

C. Tibia nigrn-anulatæ; seu basi et apice magis rel minus lulescentes.

… Tarsi articulis apicalibus, salten duobus, nigris.

1I. Scutclltm, margini setulis nigris destitutum, sed totumluteo-pilostmu rel pubescens.

Sp. 6. Fraterna Mgn. 
$1 \% 0$

HH. Scutollum, margine senlis alinuibus protitn, nigris, et distinetis a puliscentia rel pilis lutpis.

I. Tarsi ommes non ommino nigri, vel basi vel inferne ruftscentes, sultem iullmelii.

K. Epistomizm in metlio non productum, inde facirs tuberculo unico distinct"; prominentiis rufesceutibus, salten in fuemina.

\section{Sp. 7. Testaciconis Mihi.}

KK. Epistomium in medio productum, inde facies butulerculata; prominemais lateralibus non rufecentibus.

L. Antermo, salten articulo tertio, in utroçue sexu, lifiscentes. - Maris, pili frontales toti nigit. - Fxmince abdomen hive subrotundatum.

sp. 8. Venwalis fiall.

LL. Antenne in utroplie sexu nigricantes. - Hario, pili frontales nigri, Inteis permixli. - Fomince abdomen elongatum.

Sp. 9. Gunins Mihi.

II. Tarsi ommes omuino niyri, ellam basi et inferne.

Sp. 10. Liteiconas Zett.

go. Tarsi, saltem antici, articolo apicali unico n: dentibus aliquibus !uleis vil rufescentibus.

i. Thorax ut scutellun pilis, vel pubescentia laten-grisea tectus.

Sp. 11. Precux Zell.

ji. Thorax dorso aigro-ploso, 11 scutel!um.

Sp. 1‥ Pestiol, Wihi. 
GG. Tibice omnes vel totæ nigrœ, vel basi tantum paulo Iutescentes.

M. Tarsi vel Ino vel quatuor anteriores, in medio pallide lutescentes, basi nigricantes.

\section{Sp. 15. Fuvinana Mgn.}

M11. Tarsi omnes etiam in medio nigri, vel nigrieantes. N. Antennce articolo tertio rufo vel rufescente. Sp. 14. Innupta Zelt.

NN. Antemno tolæ nigræe vel nigricantes.

O. Tibic ad basim picex vel rufescentes.

$$
\text { Sp. 15. Coragina Zett. }
$$

00. Tiliœ etiam basi nigræ.

$$
\text { Sp. 16. Variabilis Fall. }
$$

AA. Oculi etiam in maribus (notis) nec pilosi nec manimanifeste pubescentes.

P. Pedes toli nigri.

Q. Arista vix prope basim pubescens. - Frons, Thorax, scutellum el abdomen, pube sub-aurea brevi non tecta. Sp. 17. Nigripes Mgn.

QQ. Arisia prope basim distincte pilosula. - Corpus superne pube sub-aurea brevi tectum.

Sp. 18. Pedenontana Bellardi.

P!'. I'edes partim litescentes.

R. Tarsi omnes saltem superne nigrica nies. 
S. Antennœ articulo tertio mediocri.

$$
\text { Sp. 19. LAtifbors Zett. }
$$

SS. Antennœ articulo tertio amplissimo.

$$
\text { Sp. 20. Laticormis Mihi. }
$$

RR. Tarsi, saltem quatuor anteriores, magis vel minus late rufescentes, vel lulescentes.

T. Arista prope basim distincte pilosula. - Scutellum in fominis apice plus minusve lutescente.

U. Antenno maris articulo tertio nigricante-fusco, extrinsecus lutescente. - Os faminæ maculis latcralitus distinctis fusco-luteis; maris minus apparentibus.

$$
\text { Sp. 21. Scutellata IJgn. }
$$

UU. Ancennce maris articulo tertio fere toto luten. Os fominæ et maris, lateribus non lutei maculatis.

Sp. 22. Soron Zett.

TT. Aristz etiam prope brasim subnuda. Scutellum in utroque sexu nigrum unicolor.

$$
\text { Sp. 23. Subalpinal Mihi. }
$$

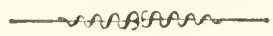

Observat: el Symonimia

Sp. 1. C. Oestracea Lin. Falr. Lalr. Mgn. Malq. Loëro, Rupestris Fall.

v. Descript. Zetlerstedtii. 
Species non rara in Pedemoutiu: a Mussino Trupui et Bellardi mihi communicata.

Sp. 2. C. Grossı Fall. Mgn. Macq. Wulk. Zett.

Synon: Zetterstedtii dubia, quia abdonien lateribus tantum pilis rufis hirtum dicitur, ct albedo palporum ab ipso notala, in exemplaribus meis non observari.

v. Descript. Macquartii.

Abdomen maris, una vice taltum lecli, inferne, et non superne at apicem nigro-pilosum.

Fœm. non rara in agro parmensi; eam in planilie et in collibus primo vere inveni, mensibus martio et aprile: In Pedemontio quoçue capta a Bellardi.

Sp. 3. C. Aunata Fabr.

Caniclearis Mgn. Macq.

V. Descript. Macquartii et Mcigenii, quubus adde, saltem pro exemplaribus Italicis.

Tibie sxpins anu!o fusco signatx. - Thoracis fascia fusca aliquando non manifesta.

Antenme frequenter, totæ fusco-rufescentes.

Frons fuivo-pilosa, vertice pilis nigris.

Sp. in agro parmensi rard: a me semel capta in collibus, mense martio; el bis a Doct. Bertèo in apenninis: cotcra exemplaria collectionis meæ pedemontana, ubi non rara videtur, nam nounulla specimiua a Geneo Mlussino et Bellardi obtinui. 


\section{4}

Sp. 1. C. Flavires Pamz. Mgn. Kell. Wall.

V. Descript. Zetterstedtii.

Species in llalia rara: bis tantum a me rapta in collibns subapenninis agri parmensis, el scmet ex P'edenumio a Bellardi missa.

sp. 5. C. Flaviconnis Fabr. Líssi. Mgnn. Macq. Zelt.

v. Deserigh. Meigenii.

synonim Zetlerstedtii dubia, quia anths fuseus tibiartins the quo lountulur $\mathrm{Cl}$. Auct. in nostris exemplaribus seinper derst.

Riaro capta a D. Berlio in apenuinis, ef a me in contisus ditionis parmessis, ubi copulatam semel inveni.

Sp. 6. C. Fraterina Algn. Zell.

V. Deseript. Zetlerstedtii cui adde.

Pili fromtales (in mare) retro antemnas toti nigri. - Froms ixcavata.

(la uroque sexu) sculpl'um margine setulis nigris dostituman, inter pilos, vel mubscentian pallide grisescentes.

Pedes libiis late nigno-anulatis, latsis intermediis ima basi, et ommes inferne filleseentes.

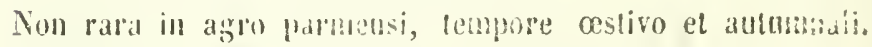

- Ia Etriria linoque eapta a D. Piecioli.

Sp. 7. Ch. Testaciconas Mihi. (Fomina).

Similis fomine C. Fraterme, sed satis diversa. 
Antennis articulo tertio rufu-piceo, lato, et prinis duobus nigris. - Facie luberculo unico distincto, quia cpistomium in medio non productum. - Ore prominentiis Iateralibus, apice testaceo-rufo.

Alii characteres speciei sunt. - Arista subnuda. - Oculi pilosuli. - Pedes tibiis fulvescentibus, anulo lato nigricante; tarsis anticis el posticis superne nigricantibus; intermediis, articulis duobus vel tribus primis subfulvis elc.

Raro focminam invenit Mussino in Liguria.

Sp. 8. C. Vebnalis Fall. Mgn. Macq. Zelt.

v. Descript. Zetterstedtii.

cui adde. - Antemœ etiam articulis primis rufescentibus. Non frequens legitur in agro parmensi priesertim, ab aprile all Junium.

Sp. 9. C. Gulkixa Mihi. Longit. Mill. fere 9.

Similis. C. Fraterne sed distinctissima.

In ul!ropine sexu scutclli margine setulis nigris instructo, priter pilos vel pubescentian.

In sarre, Fronte non distincte cxearata, et pilis nigris retro antennas, Jutescentibus permixtis; net, non antemnis uigrioribus.

Foeminie artieuhs tertius antennarm siepins ctiam fuscior, sell picens, sed alipquando rulescens.

In collibus sub-apenninis mimo rere non infrequens, in 
ditione Parnensi. - In Etrmia quogute lecta a Doct. Piccioli.

Sp. 10. C. Lutcicorns Zett.

v. Descript. Zetlcrstedtii.

Ab affuibus præsertim distincta; tarsis totis et omnibus atris etiam inferne, et tibiis late nigris, apice angustissime, et basi latins fulvescentibus.

In collectimcula Pedemontana ab Equite Truqui missa, fœminam speciei inveni.

Sp. 11. C. Precox Zeit.

V. Descript. ejusdem Auct.

Frequens in collibus ditionis parmensis primo vere, in floribus præsertim ranunculorum, sed usque ar junium legitur. - Etiam in Pedemontio labitat, ubi eam invenit Bellaidi.

\section{Sp. 12. C. Pusilla Mili.}

Omnium nostratium uninor. Longit. Mill. 7.

Maris, Antennœ a:rœ. - Fucies nigra, prope oculos paulo albidi pollinosa. - Frons ut vertex nigra, nigro-pilosa. - Oculi hirli.

Thorax niger paulo olivaceus, nigro-pilosus.

scutellum nigrtun paulo oenescens, nigro-birtum.

Abdomen nigro-opacuin, apice, et lateribus interrupte nitidis: pilis tateralibus lungis, pallicte lutescentibus. 
Halleres stipite pallido, clava nigricante.

Pedes nigri, anticorum et internediorum tibiis, basi et apice Jutescentibus; tarsisque interntediis, et aliquando etiam præcedentibus in medio panlo luteis: posticorum geniculis tantum lutescentibus.

Alce paulo infuscatæ.

Fœı: levis nitida. - Halteres toti pallidi. - Pedes magis late lutescentes, tibiis posticis, apice quoque anguste luteo. - Oculi brevissime pilosuli.

Rara in locis montuosis ditionis Parmensis, a majo ad septembrem.

Sp. 15. C. Flavimana Mgn. Loëzo. Zett.

Videa Macq.

v. Descript. Zelterstedtii.

Sp. satis fræquens in agri parmensis planitie et locis montuosis, præsertim mensibus majo et junio. - Vivit quoque in Pedemontio et in Etruria, ubi eam legerumt Bellardi et Pechioli. - Exemplar ejusdem eliam n Spinola misit, in Corsica inventum.

Sp. 14. C Inyupta Zett.

v. Ejusd. Auet. descript.

Ut Clar. Zetlerstedlins, pgo quoque fouminam tantum spresiei inveni, rarissimo in agro prarmensi. Forte mas cum nuaribus alie speciei confusus. 
$1: 8$

Sp. 15. C. Conachia Zell.

V. Ejusd. Auct. Descript.

Fominam tantum observari in apennino parrensi lectam a Doctore Bertèn.

Ista ab aliis fominis specierum afinimm, oculis hirtis, et pedum colore omnino nigro, excepta tibiarum basi non anguste rufescente, distincla.

sp. 16. C. Vapiabus Panz. Fall. Mign. Macq. Lö̈le. Zett. Atra el Nigrita Falor.

V. Descript. Zetterstediii.

Satis rarn capta a Doct. Beplèo et a me in monlilus ritinnis Parmensis. - Marem speciei a P. Mussino accepi, prope Augustam Taurinorm inventem.

Sp. 17. C. Nigntpes IIgn. Macq. Walli.

ScIn⿴囗十) Zell.

v. Descript. Mieigenii.

Non î requens in agri parmensis planitic el collibus, presertim mense majo, in filn copulan speciei inveni. Semel in apennino capta a D. Bertèo, et in Etruria lecta a D. Piccioli.

Sp. 18. C. Pebemontana Dellardi (in lilleris) Longit. Hill. fere 8.

Fominam tantum specici mihi Prof. Bellardi transtinisit, "luas 
characteribus sequentibus ab aliis salis distincta videfur.

Aurenne cum arista nigerrimx, arisła dislincte pilosula.

Zuacies nigra Ballide lutei pollinosa, tuberculis el spatiis

dunbus lateralibus, denudatis, levibus.

Frons nigra, paulo olivacea, pube sub-aurea tecta, vertice nigro-piloso.

Oculi non manifeste lomenlosi.

Thorax, scutilhum, abdominisque dorsum nigro-olivacea, pube sul)-atrea, brevi, tecta.

Pedes omnino alri ul latera thoracis.

Ata grisea hasi lutescentes.

A Cheilosia putbera nondum in Ilalia capta, cui proxima est, difert prisertim arista pilosula, el pubsecentia corporis sub-aturea.

rara in Pedemontio habitat.

Sp. 19. C. Latrirnoss Zelt.

V. Discripl. ejusd. Auctor

Frominas speciei nonnullas in collibus dilionis parmensis inveni, et marem una vice lantum, gliem dubilantre huic refero Cleilosix, nam ejusdem oculi manifeste pilosi sunt: et si cerle mas iste latifronti peitinet, tunc sprecies in sectione oculis lirlis locanda.

In boc mare Antenue prarre nigricautes.

lirons ut vertex nigin-pilasa. - Halteres clava dilute fiesca.

- Abromen bateribus sordide lutesernte-pilasis, al

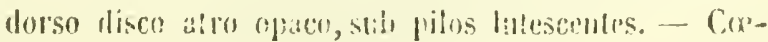


tera II in fomina a Zeterstedtio descripta, exceptis characteribus sexualibus, oculorum, fronlis elc.

Sp. 20. C. Laticennis Mihi. Longit. Mill. 11.

Pro unico exemplari focmineo, in parmensi apennino capto a D. Berlèo, specienı instituo, sic ab aliis distinctam. Antennee arliculo tertio amplissimo, piceo.

Oculi omnino nudi.

Frons et Thorax nigricantes, litei pubescentes.

Abdomen uigro-subcertulescens, pilis albidis præsertim oad latera instructum.

Pedes nigri, tibiis hasi et aprice rtifescentibus.

A Cheil. Chalybeata Meigenii, cui affnis est difert; latitudine articuli terlii antennarum, el tibiis non solum ad basim, sed eliam ad apicrm rufiscentibus.

Sp. 21. C. Scetelesta Fall. Mign. Macq. Zell. Loëu. Mndh. Walk.

V. Descript. Zetterstedtii.

Vuigalissima in agro parmensi, tam in planitie quam in montunsis, a mense aprile ad seplembrem. In Pedemontio a Bellardi, in Etruria a D. Piccioli, in Insubria a Fratribus Vilta et a Comite D'Ar'co. Bononir a Bertolonio, in Regno neapolitano a D. Costa lecta. A P'upis hujus speriei, in Bolelo Lurido a me inventis, insecti declarata domi orta. 
Sp. 22. C. Sонов Zett.

V. Descrip. ejusd. Auct.

Vulgaris, cum præcedente in agro parmensi, iisdem temporibus et locis invenienda.

In Etruria lecta a Picciolio, in Insubria a Villa etc.

Sp. 23. C. Subalpina Mihi - Longit. Mill. 7-8.

Maris Antennce mediocres, fulvæ, paulo fuscescentes, arista vix puberula, non pilosa.

Facies nigra, levis, nitida, vix prope oculos albidi pollinosa. Frons nigra, nigro-pilosa, prope oculos albicans. Oculi nudi omnino.

Thorax ut scutellum nigro-nitidus, et pilis nigris hirtus. Abdomen nigro-nitidum, excepto margine postico segmentorum intermediorum late nigro opaco; marginibus albicante-pilosis.

Halteres toti albido-lut escentes.

Pedes femoribus nigris, summo apice Juteo; tibiis luteis, anulo intermedio fusco: tarsis luteis, omnium apice, et anticorum et posticorum etiam basi nigricantibus.

Fœm. Antenna magis pure fulvæ, articulo tertio satis latiore.

Frons ut thorax nigro-pubescens.

Abdomen atrum nitidissimum, cœtera circiter ut in mare. In collectione Mussino marem speciei inveni, alterum sexum Pr. Bellardi a Pedemontio misit.

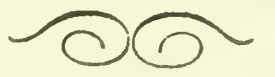


Geume 21. ORthoverta Macq. Rndn. Walk. Emistaris Fall. Ceraxsugaseme Mgn. Loëro. Zett.

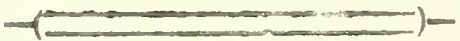

\section{Cuaract. Generici.}

Antennce articule tertio elongato-sub-ovato, et apice palo acuminato; arista nuda, propo basim articuli inserta.

Oculi nudi, in fronte maris angustissime subcontigui.

Frons in mare puncto impresso distincto, in fomina transversim et seriatim sulciolata.

Facies in utroque sexu, nec tuberculata nec carinata, sed distincte concava, epistomio modice producto.

Ale vena quinta longitudinali angulatim cubitata, et supra cubitum intus modice flexa, ita ut venæ quartæ cui apice conjungitur sub perpendicularis sit.

Species Italiea

Sp. 1. Nosius Fall.

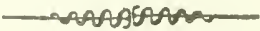

Observat. et Synonim.

Sp. 1. O. Noslus Fall. Mgw. Macq. Zatt. Rndn. Walk. V. Descript. Zetterstedtii. 
Species non rara in planitie et collibus ditionis parmensis $\left({ }^{*}\right)$.

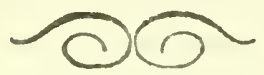

\section{Conum a*. GaMpligera Rndr.} Oathoneuna? Loëzo.

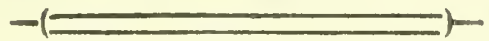

(") Species congeneres, Meigenii, distinotæ a nobili, vel colore pedum flavescente, vel forma antennarum, Buropam bahitantes, sed nondum in Italia captæ, si in posterum appd nos inveniantar, sic metodice distinctæ erunt.

A. Pedes toti nigri,

B. Thorax niger non æuescens.

Sp. 2. Nigricoruls Mgrl.

BB. Thorax ænescens.

Sp. 3. Noatus Fall.

AA. Pedes geniculis ot tarsis, saltem anticis, partim lutescentibus.

C. Antennce elongatæ, articulo tertio longo sublineari.

Sp. 4. EuEouss Wiedm.

CG. Antennae mediocres, articulo tertio paalo dilatato, non lineari.

$s p$. J. Geniculatm Myn.

Specis Loëmii, Plumbago, Brevicornis Frontalio, mibi ignots. 


\section{Charact. Gener.}

Antenne articalo tertio breviter sub-ovato vel fere subquadralo, arista dorsaali nuda, prope basim articuli inserta.

Oculi uudi, in fronte maris modice late contigui.

Frons in masculis puncto aullo impresso; in fominis seriatim suiciolata.

Facies in utroque sexu in medio satis concava, nec tuberculata nec carinata, epistomio distincte producto.

Ala vena quinta longitudinali modo inusitato intus flexa, ita ut pars cubitata ubi conjungitur præcedenti, angulos duos sat diversos efficiat, interiorem satis obtusum, exteriorem satis scutum.

Species Italica. Sp. 1. Venusta Mihi.

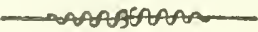

Observat. et Synonim.

Sp. 1. C. Venusta Rndn. (Genera Ital. Ord. Dipt.)

Longitudo Mill.-š-circiter.

Maris Antennce nigræ, articulo tertio piceo.

Facies nigricans prope oculos paulo albida.

Frons nigra, albidi pilosa.

Thorax scutellum et abdomen nigro-œnescentia nitida, albidi paulo pubescentia. - Scutello et abdominis apice obscurioribus. - Abdomine breviter ovato.

Caliptera alba. - Halteres albicantes. 
Pedes toti et omnes nigri.

Ale paulo fuscie, basi sublutescentes.

Foun: Abdomen magis rotundatum, subdisciforme, frons haud albopilosa, cætera ut in mare.

species satis rato lecta in apennino parmensi a Doct. Bertèo $\left({ }^{*}\right)$.

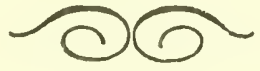

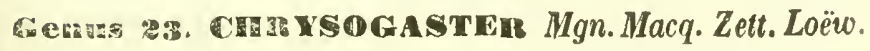
Rndn. Walk.

Sunphes Fabr. Panz.

Ciststams Fall.

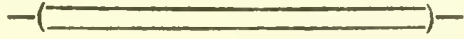

\section{Ciraragteres Generici.}

Antenna articulo tertio subcirculari, arista dorsuali nuda.

Facies in mare nunc subtuberculata seu gibbosa, nunc ut in fomina satis concava, tuberculo nullo.

Oculi nudi, in fronte maris nunc contigui, nunc paulo remoti.

Frons maris aliquando tumida et oculis elatior, aliquando plana: fomina vel transversim sulciolata, vel rugosa, vel scriatim punctulala.

Ale vena quinta longitudinali ultra cubitum sinuosa, et ubi conjungitur quarta, augulos duos efficicute subrectos, scilicet interioreus et exicriorem subaqualiter apertos.

( Nola) Differentia characterun faciei fronlis elc. in diversis specichus Chrysogastri, forte genertum aliquorum

(i) Orthonenre aliqua Loewii, affinis vel similis? 
separationem petil, quod si placuerit, ea sic distincta et. nuncupata propono.

a. Facies maris supra epistomium tuberculata vel gibbosa.

- Frons in eodem sexu tumida et oculis elatior. Oculi in fronte contigui:

Faciez foeminæ concava non gibbosa. - Frons, lata lineis duabus Iongitudinalibus impressis, sulciolos laterales, sepius distinctos, in duas series sejungentibus. Antennœ utriusque sexus parvæ.

Genus Melanogaster Mihi.

aa. Facies maris, ut in foemina non gibbosa sed concava: Frons in eodem sexı plana non tumida.

Frons fœminæ lineis longitudinalibus impressis non distinctis. Antenno in utroque sexu latæ.

b. Oculi maris in fronte contigui. - Proboscis, in utroque sexu, ultra epistomium non manifeste producta. Gemus Chrysogaster Mgn.

bb. Oculi maris in fronte paulo distantes. -

Proboscis in utroque sexu, manifeste producta ultra epislomitim.

Genus Lejocister Mihi.

Species Italic

Secl. I. Melanogaster.

A. Antenne lolæ atræ. 
1. Frons fœminæ non distincte sulciolata. - Haltcres capitulo pallido.

Sp. 1. Nubilrs Mihi.

aa. Frons fœminæ distincte sulciolata. Halteres capitulo nigricante.

$$
\text { Sp. 2. Viduatus Lin. }
$$

AA. Antennce articulo tertio rufescente.

B. Ala nigricantes unicolores.

$$
\text { Sp. 3. Cemeteriorem Lin. }
$$

BB. Alce in medio fascia lata fusca, basi pallescente flavida. Sp. 4. Cunarbeatus Mgn.

Seclio II. Chrpysogaster.

$$
\text { Sp. כ. Splgndens Mgn. }
$$

Sectio III. Lejogastbr.

$$
\text { Sp. 6. Tarsatus Mgrl. ( } \left.{ }^{*}\right)
$$

Oservat. et Synonim.

Sp. 1. M. Nuburis Mihi.

Similis foeminæ viduato, et distincta, fronte rugosa, sed non

(1) Tertia sectioni cliam Chrysogastrum metallicum Meigenii adscribe Species Italicæ a me non observatæ suul; Longicornis Loëw* et Amethystina Macq. 
transversim sulciolata, et halteribus pallidis, non nigricantibus.

Semel capta primo vere in agro Parmensi.

Sp. 2. C. Vinuatus Lin. Fall. Mgn. Macq. Zett. Loëw. Walk v. Descript. Zetterstedtii.

Frequens legitur primo vere, mensibus præsertim aprile et majo in ditione Parmensi.

In Veneto et Insubria quoque vulgaris, pluria exemplaria ab hisce provinciis obtinui a fratribus Villa, De Cristofori. et D' Arco.

Sp. 3. C. Ceneterionum Lin. Fabr. Panz. Mgn. Macq. Loëw. Walk. Solstitiaus Fall. Zett.

v. Descript. Zetterstedtii.

Frequens in ditione Parmensi a majo ad Julium, et in Apenniuo etiam mense augusto.

In Pedemontio eum invenit Bellardi, in Insubria Villa, ad Tergestum Schiner.

Sp. 4. C. Chalybeatus Mgn. Macq. Zetter. Walk. v. Descript. Zetterstedtii.

Species in agro parmensi non frequens, in Pedemontio (quoque raro lecta a Bellardi. 
Sp. 5. C. Splendens Mgn. Macq. Loëwo. Rndn. Malk.

V. Descript. Meigenii et Macquartii.

Quibus adde, Antennce articulo tertio limbo, saltem superne nigricante.

Frequens præsertim in collibus agri parmensis, a martio art septembrem. - Ad Tergestum inventus a Schinero, of in Etruria a Picciolio.

Sp. 6. C. Tarsatus Mgn. Zelt.

Splendida Mgri. Mgn. Macq. Walk.

Rufitarsis Loëwo.

v. Descript. Zetterstedtii.

Raro spec. hane inveni in collibus subapenninis ditionis parmensis, supra flores bellidis perennis, mensibus ąrile et majo, et una vice mense septembre in floribus Dauci.

- Semel quoque eam accepi a D. Bellardi, in Pedemontio captam.

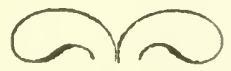

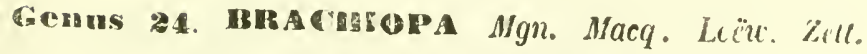
Rndn. Walk.

Omcinis Fabr.

geternoia Fall.

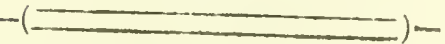




\section{Ceraract. Gener.}

Antenne articulo tertio subcirculari, arista suhnuda ad basim articuli inserta.

Facies nec carinat?, nec toherculata supra epistomium distincte productum, sed in medio satis concara: epistomio tamen non longissimo, nec rostriforme.

Oculi nudi, in fronte maris angustissime contigui.

Frons fominæ haud transversim sulciolata.

Ala satis longæ, vena quinta longitudinali quartæ ad apicem conjuncta, satis extra apicem tertiæ: angulo apicali inter venas quartam et quintam satis aculo.

Pedes femoribus posticis modice incrassatis, et subtus brevissime serrulatis.

(Nota) Cum Brachiopis arislam plumosulam præbentibus genus distinctum institueadum est, sub nomine.

Exocuella Mihi. Cujus Typus B. Ferruginea Mgn.

\section{Species Italica}

A. Ale immaculatx.

Sp. 1. Bicolog Full.

A. Ale fusen bimaculatie.

Sp. 2. Arcuata Panz. 
Observat. et Syronim.

Sp. 1. B. Bicoror Fall. Mgn. Macq. Zett. Loëzo. Walk. Rndn.

จ. Descript. Zetterstedtii.

Non frequens in agro parmensi, a me raro lecla in planitie tantum propepadana.

Sp. 2. B. Arconta Panz. Mgn.

V. Description: in opere Meigenii.

Unicum exemplar detritum hujus speciei, collectionis D. Mussino mihi notum erat, quando specimina duo a D. Schiner accepi, prope marem adriaticum capta, ad Tergestum.

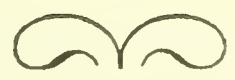

Genus 5. RHYweila Scopoli. Fabr. Rossi. Panz. Full. Mgn. Macq. Loëw Zett. Curlis. Rndn. Walk.

Charact. Generici.

Generis bujus characteres plures ut in Brachiopa, sed ab sadem distiuctum.

Femoribus posticis non incrassatis, et Oculis marium in fronte litte cobereatibus: ab omnibus vero geaeribus hujus stirpis difert. 
Epristumio valde elongato, rostriforme, et poubosude epistomii long $\mathrm{i}$ \{ndine, apice non distincte labiata.

\section{Siecies Italice.}

A. Abdomen lateribus, et margine postico segmentor: quarti, et puinti late niglis.

\section{Sp. 1. Campesthis Mgn.}

Ad. Aldomen testacenm, nec lateribus, nec marginibus segnentorum quarti el quinti nigris; aliguando tantum linea exili ad marginem posticun segmenti quarti nigricante.

\section{Sp. 2. Rostrata Scor}

\section{Observal: et Synomimia}

Sp. 1. R. Campestris Mgn. Macq. Zell. Curtis.

v. Descript. Zetterstedtii.

Cui adde: Distincta a Rostrata Scopolii, præsertim lateribus ábdominis, etiam in segmento quarto nigris.

Species salis rara apul nos, eam legi aliquando in collibus subapennius dilionis parmensis, et ejudem unicum excmplar a b). Bellardi accepi, in fedimontio captum.

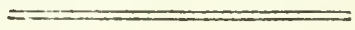


Sp. 2. R. Rostrata Scop. Lin. Fabr. Rossi Panz, l'all. Mgn. Macq. Loëz. Zett. Rndn. Waik. a Walk. Synonim: exclude Campestris Mgr.

v. Descript. Zetterstedtii.

A mense aprile ad julium legitur, fræquens in planitie et montuosis agri Parmensis: in Pedemontio quoque eam sæpe invenit Bellardi.

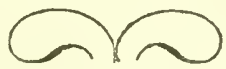

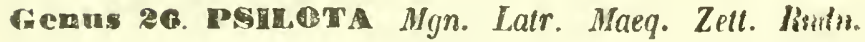
Walk.

Characteres Generici.

Antenna capite transverso breviores, articulo tertio ovato; arista nנ da ad̉ basim articuli inserta.

Facies nec concava nec distincte taberculata; epistomio tantum pauto prominulo.

Oculi hirti, in fronte maris late contigui.

Abdomen vix aut non longius thorace cum scutello.

Alce vena quinta longitudiuali angulatim nexa, a culito ad mpiem recta, prope apicem alarum quirtæe conjoncta, et cum ipss angulum interiorem non valde aculum eficiente: sexta lomgitudinali angulatim cubitata et precedenti ad apicem cosjusteti, satis longe a cubito ejusdem. - Tena spuria non masifesta. 
Pedes femoribus posticis paulo inerassatis, tibiisquo aliquantulem incarvis.

Species Italica.

A. Tarsi omnino nigri.

Sp. 1. Conjugata Miki.

A Tarsi basi distincte flavida.

Sp. 2. IsสUPTA Mihi.

Observat. et Synonim.

sp. 1. P. Conjugata Mihi. - Longit. Mill. 6-7. Amturacina? Macq. sed non Meig. Valkerii antracina $\mathrm{Sp}$. altera.

Diagnosis brevissima Macquartii Ps. Anthracinæ, \{ a Meigenio forte escripta), quia insecti a $\mathrm{Cl}$. Auctore non observati; nam silet de sexu, et icon ejusdem non perfecta. Veseriptı Meigenii de hac Psilota, fominæ tantum pertinet, sed exemplaria nostra foeminea a Meigeniana diversa, colore antennarum, articulo tertio semper rufo, limbo tantum superiore nigricante, et si icones ejusdem bonæ, tunc distincta, angulo quoque interiori, a venis quarta et 
quinta longitudinalibus efformato, in nostris satis minus acuto: facie etiam differt perpendiculari, non distincte concava.

Inde mea species diversa, et eam tamquam novam describo Mas. Nigro-sub-cœrulescens, cujus.

Antenno et arista nigræ

Facies nigra, levigata, parce pallide pubescens.

Frons cum vertice nigra, parce nigropilosa.

Thorax ut scutellum nigro-lurtus.

Abdomen nigro-hirtum, exceptis lateribus ad basim et apicem albido-pilosis.

Caliptera flavescentia - Halteres lutei.

Pedes nigri nigro-pilosuli, tibiis quatuor anterioribus bas ${ }^{\mathrm{i}}$ fusco-rufescentibus vel piceis: tarsis posticis pube flavida inferne præditis.

Alœ limpidæ, basi vix lutescente, et vitta stigmaticali fuscoflavida.

Fom. Frons antice, et facies albillo-pubescentes. Antenna articulo tertio rufo, limbo supkero tantum nigricant?. Abdomen fere totum albo-pilosum; cotera circiter ut in mar.

(Nota) Quamvis numquam copulata, exemplaria utriusçue scxus hic descripta, tamen iisdem locis et temporibus, imo in iisdem floribus semper inventa, ideo earun differentias, specificas non esse, sed sexuales credam, nisi mares antennis inferme rufs, vel fominas antennis totis nigris inveniantur.

Species non raro a me lecta in collibus ditionis parmensis, primo vere, a martio ad majum, supra flores Pruni, Oxyachantæ, et nonnullarunı umbellatarua. 
Sp. 2. P. Innupta Mini Longit. Mill. 6. Anthracins? Walk.

Fœm. Nigra œnescens, non cœrulescens præsertim in abdomine Antenna ovatæ longiores quam in femina conjun ctae, et totæ nigræ.

Facies et frons nigro-nitidæ, albo-pilosulæ.

Thorax dorso nigro-pubescente, lateribus albo-pilosis.

Abdomen albidi pubescens.

Caliptera albicantia. - Halteres Intei.

Pedes nigri, albido-pilosuli, geniculis tibiisque saltem quatuor anterioribus basi rufescentibus: tarsis omnibus articulo basali rufescente.

Alœ in medio fusco-lutescentes.

Fœminam tantum speciei mense aprile inveni, in collibus suhanenninis ditionis Parmensis, in floribus Pruni padi.

(Nita) Psilotœ nonnulli Zetterstedtii, ocults nudis, generi distincto alscribendæ, quod apellabitur LEJoTs. Mihi.

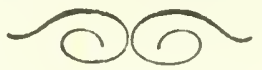

Genus 2\%. (A) ThIGETPEOS Loëw. Rndn.

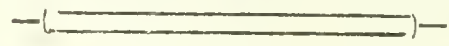

Charact. Genek.

Anlenno brevissimæ, articulo tertio leaticulari; arista dorsuali nuda brevi. 
Facies nec excavata, uec tuberculata, nec carinata.

Oculi breviter birti, in fropte maris non angusto contigui. Alce ut in genere Pipiza renos

Abdemen segmentis secundo et tertio latissimis, modo oxtraordinerio. Pedes uon incrassati.

(Nota) Nunc exemplaribus speciei typicæ a me observatis utriusque sexus, genus Loěwi adoptandum esse cogito.

Species Italica

Sp. 1. Prince Lohio.

Observat: et Synonim.

Sp. 1. T. Prigus Loëro.

v. Deseript. ejusd. Auct. (Bemerk. Zur Eutomol. 1840). Rarissima species, in Italia. A me capta in planitie parmensi supra flores Jasmini; fæmina bis mense augusto; semel mas mense Julio. - In collibus una vice marem inveni mense majo labente in floribus Ligustri vulgaris. In Etruria quoque semel capta fœmina a D. Piccioli.

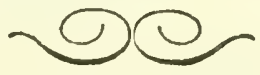

Genus 27. (A) PIPIEn Fall. Mgn. Macq. Zett. Curtis. Loèw. Rndn. Walk.

Sxнpuve Fabr. Rossi. 


\section{Charact. Gener.}

Antennce articulo tertio vel ovato, vel sub-obtruncato, vel fere subquadrato; arista dorsuali nuda.

Facies omnino plana, nec carinata nec gibbosa.

Oculi pitosi, in fronte maris non anguste contigui.

Abdomen segmentis tribus intermediis; modice et subæque latis.

Alce vena longitudinali quinta, ultra cubitum intus incurvata, et cum quarta ad apicem, angulum interiorem salis acutum efficiente: excepta Sp. Obscura, in qua angulus minus acutus:

Prima longitudiaali ultra transversam interiorem fraulo producti. Pedos femoribus posticis paule incrassatis.

\section{SPeciei Italica}

A. Abdomen flavo-fasciatum, vel maculatum.

B. Ala in medio nigricante-fasciatæ vel maculatæ, vel saltem distincte infuscatie.

C. Tarsi omnes omnino luteoftlvi.

Sp. 1. Noctiluga Lin.

CC. Tarsi omnes saltem apice, vel postici etian basi nigricantes.

D. Alce vena transversa anteriori ante apicem sita longillıdinalis primæ, et non perfecte contra apicem. Abdomen segmento penultimo, lateribus et limbo poslico subreflexis.

Sp. 2. Geniculata Mgn: 
DD. Ale vena transversa anteriori, perfecte contra apicem sita longitulinalis primæ. - Abdomen segmento penultimo, lateribus et limbo postico planis, non subreflexis.

$$
\text { Sp. 3. Plana Mihi. }
$$

BB. Alæ nec fusco-fasciatæ nec maculatæ.

$$
\text { Sp. 4. Excalceata Mihi. }
$$

A A. Abdomen unicolor.

E. Alce nec fusco-fasciatæ, nec maculatæ.

F. Tarsi quatuor anteriorts, apice excepto, lutei. - Tibics intermedix !nberculatæ.

Sp. 5. Vitripenwis Mign.

FE. Tarsi anteriores nigri. - Tibiœ intermediæ non tuberculatæ.

G. Pedes non omnino nigri, saltem geniculis et metatarsis intermediis lutescentibus.

Sp. 6. Obscura Macq.

GG. Pedes omnes omnino nigri.

$$
\text { Sp. 7. Vidua Mlihi. }
$$

EE. Ala nigricante-maculatx.

Sp. 8. Lugueris Fabr. 
Observat: et Symonim.

Sp. 1. P. Noctiluca Lin. Fubr. Fall. Walk. (Non Zett.) Mgu? Macq?

(fnem.) Festiva Mgn. Macq - Fasciata Mgn. Macq. Lunata Mgrl. Mgn. - Rosarui Panz.

(Mas.) Luteitansis Zett.

In sectione Pipizarum, alas in medio infuscatas, et abdomen lutei maculatum præbentium, $S_{p}$ : ista distincta est, præcipne tarsis totis et omnibus fulvo testaceis, ut in icone Panzerii, ab omnibus citata ubservatur.

Variat plurime forma, latifudine, et etiam numero maculalarum flavarum abdominis, et colıre quoque nunc magis nunc minus flavescente vel rufescente, inde species nonnulle e varietatibus conditx fuerunt.

Synonimia Meigenii et Macquartii dubitanter inclicata fuit, quia in diagnosibus eornm P. Noctilucte, maris facies nigropilosa dicitur, dum in nostris a me observatis, pallide-Inteis pilis, ut in fœmina tecta est.

Sp. non rara sed non vulgaris in Iralia. - In ditione parmensi eam legi mensibus martin et majo in planitie et collibus: et prope Mantuam D'Arco - et prope Florentiam Piccioli, exemplaria non nulla legerunt, et miserunt.

Sp. 2. P. Geviculata Mgn.

Signata Mgn. (Fom. var.)

Nocticees Zell. (esclus. Synon.) 
v. Descripl. Zellerstedtii P. Noctiluex, que Géniculata Meigenii perfecle convenit; el ista diagnosi adde, ut a sequente distiucta sit. Alarum venula transversa anterior, paulo sed distincle, ante apicem primæ longitudinalis locata; et abdominis segmentum penultimum, lateribus et margine postico subreflexis.

Bis primo vere uterque serus legi in planitie parmensi, et semel in Apennino, mense julio.

\section{Sp. 5. P. Plana Mihi. Longit. Mill. 6 (fœm).}

(font.) Excepia parvitate, similis habitu P. Geniculato, tamen angustior et distinctissima, abdominis segmentis, præsertim peuultino, nec apice, nec ad latera subreflexn; et remularum intermediarum anteriore, perfecte contra apicem, et non ante apicem primæ longritudinalis sila. Rarissimo lecta fomina tempore verno, in collihus subapenninis ditionis parmensis.

Sp. 4. P. Excalceata Mihi. - Longit. Mill. ?.

Nigro-subnenescens, tota albido-luirta.

Antennce articulo tertio subquadrato, nigro, inferne macula rufa notato.

Caliptera alba. - Ilalteres trstacei.

Abdomen segmento secundo, maculis duabus ovatis, distantibus, fusco-testaceis.

Pedes femoribus nigris, summo apice luteis, tilitis luteis, late nigricante-anulatis, tarsis anterioribus totis, et posticis, melatarso excepto, lutei-fulvescentibus. 
Alæ sublimpidæ, vitta marginali lutesceute.

Rarissima in agro Parmensi: femina lantum a me capla.

Sp. 5. P. Vitripennis Mgn. Macq.

Synon. Walkerii dubia, quia tarsos speciei fulvos esse dicit, dum certe in mare, tarsi ommes apicem nigrum præbent, et postici fusci sunt, fulvi tantum pubescentes.

V. Descript. Maigenii et Macquartii.

Sed observandum est, Macquartium non loqui de fœmma, et Meigeninm de differentiis sexualibus silere: forte nıres tantum, ipse quoque cognovit.

Unus vero et alter tacent de colore articuli tertii antennarum, inferne ruf.

Sed ego tria exemplaria maris, et unum foeminæe observavi, et in omnibus tertius antennarum articulus, macula rufa infera signatus est. - In fomina ut in mare tibiæ intermediæ prominentia præditæ sunt, quamvis in maribus magis perspicna, et tarsi in specimine fomineo a me observato magis obscuri, metatarso tuntum in quatuor anterioribus luteo, et in posticis fusco, pube fulva tecto.

Raro a me lecta in collibus et planitie ditionis Parmensis mense majo.

Sp. 6. P. Obscura Macq.

V. Descript: ejusd. Auctor. 
Species mihi ignota, in Sicilia lecta teste Macquartio; quæ antemis huic generi pertinet, et alis Pipizella.

Sp. †. P. Videa Mihi. - Longit. Mill. 9.

Tota atra, nigro et fusco-pilosa.

Caliptera cum cilis nigricantia.

IIalteres stipite fusco, capitulo sublutescente.

Abdomen nigro-pilosum, pilis pallidioribus subfasciatum;

segmento secundo maculis duabus ovatis cinereis,

certa !uce observatis, distinguendis.

Pcdes geniculis anguste piceis, tarsis pallide lutei pubescentibns, alibi pilis nigricantibus et pallidis præditi. Ala sublimpidæ, vilta marginali fusco-obscura. Mras speciei semel a me inventus, in colle sub apennino ditionis parmensis.

Sp. 8. P. Lugubris Fabr. Zett.

(Synon. Meig. Macq. et Walk. dubia).

v. Descript. Zetterstedtii.

Non rarissima in agro parmensi, ubi legitur mensibus majo et junio.

In pedemontio quoque eam invenit Bellardi.

(Nold) Pipiza qualuormaculata Panzerii, Fall: et Meig: cujus forte varietas est Quatuorguttata Macquartii, nondun 
inventa fuit apud nos, et si in posterum inveniatur, Iunc in divisone propria, ante alias locanda, simul cum $f a-$ seiata Meigenii; quæ sectio distincta erit, abdomine maculis quatuor luteis notato.

Nonnullæ aliæ species auctorum in Italia non reperlæ, sed pro certo habeo aliquas varietates esse, et non species, carachteribus consideratis parum firmis, quibus eartum distinctio innititur. - Ab istis exclude P. Obscura Macq. Sp. bona.

Species Chalybeata Mgn. in Sicilia inventa teste Macquartio, mihi ignota.

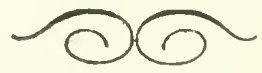

Gewan 88. HenYwGiA Rndn.

Pipiana Zett.

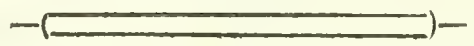

Craract. Generici.

Ala eaput pedes eirciter at in genese Pipiza.

Antenna articulo tortio valdo elongato, ut in genere sæquenie Pipizella. 
Species Itraluca Sp. 1. ZвтTEL:Tert! Iirulu,

Observat: et Synonimia

Sp. 1. H. Zetrerstedtu Rindn.

Piptza Heryngii Zell.

v. Descript. Zetterstedtii。

Non rarissimo in agri parmensis planilie ef collibus a nie lecla, mensibus niartio et aprile.

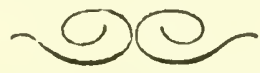

Gentus 20. PlPizelda Rndn.

Pipiza Hall. Mgn. Macq. Zelt. Rndn.

Sxupnus et abeio Fabr.

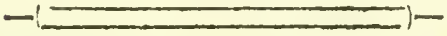

Antenne articulo tertio satis elongato, arista dorsuali nuda, prope basim articuli inserta.

Facies piana, nec tuberculata nec excavata, epistomio tantum pauls prominulo.

Alc rena quiola longitudinali ulira cubitum sinuosa, et ad apicens cum quarta cui conjungitur, angulum iuterioren subrectum efficiente: prima longitudinali costalcm attingente, vix cunts aut anle transversam exteriorem, et non ultra.

Coters ut in pipizis. 
Oisprvat. et Synonim.

E. 1. P. VARons Virdn. (Anuali di Bologna 1848.)

lis hate specie, nommullas anctormm coacelvavi, quid characteribus imnixas parmm firmis, coloris pedum et alarum, maxime variahilis.

Aisi ommes, cerle phures sienumlimm, varianti mex referembe simut.

i'. Conuresthis Fall. Zell.

Vukess Fabr. Mgn. Loëw. Macq. Walk.

Maculapevvis Mgrl. Mgin. Zett.

Varipes Mgn. Loëw. Zelt.

Anvelata Macq. Mgn. Loëw. Zell.

Geficulata Curtis.

Si culoris lutei tarsorum et tibiarmm latitudinem, et nigredinis alarum intensilatem et ex'eusionem satis diversas, Lamfuam specificos characteres considerare veliImus, lumc pipgize supra ennumerata fere omnes in Ilalia habitan', ubi nomnullae eliam vulgares sunt. Larva specipi aphistes levorat. V. Observ. meas in diariis: Numi a!mali ali Bologma 1848 .

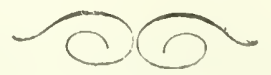




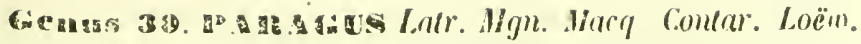
Zell. Rndn. Halk.

opgana Full. - Grupres l'anz. Rossi

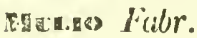

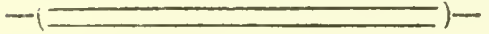

Cuaract. Gener.

Antenna articulo tertio salis longe, vel elongalo sub-ovalo; arista dorsuali nuda, procul a basi articuli iuserta.

Oruli romenloso-fasciali, iu troute maris anguste conligui, fomiua parum disliusles.

Furies non plana, sed vel paulo gibbosa, vel aliquantulum subcarillatil vel inflata.

Aler circiter ut in Pipizella venosa.

Siecies Itralice.

A. Sculellum nirstum unicolol.

B. Antema ayticulo lerlio ovato-elongato, nou satis lomgon.

- Faries in medio nigro-viltata in ulroyue sexu.

C. Tibice posticx hand nigrvicante-anmlalie.

Sp. 1. Congunates Rndn.

CC. Tibice puslice anulo niogricante mogis vel mines, seal se'mper manifesto. 
D. Tarsi postici toli rufescentes.

Sp. 2. Femoratis Mgrl.

DD. Tarsi postici articulo primo seu basali, saltem superne niglicante.

\section{Sp. 3. Tarsates Mihi.}

BB. Antenne articulo tertio salis olongato.

Facies freminat tantum, nigrovittata.

\section{Sp. 4. Pecuılul Mihi.}

Ad. Scutellum apice magis vel minus late albicante vel larescente.

E. Abdomen nigrum unicolor.

F. Tibice posticæ antulo nigricante, plus minusve sed scmper distinclo

Sp. ̈. Turduastra Fall.

FF. Tilice postica anulo nigricante non manifesto.

G. Tarsi postici toli lutescentes. - Oculi griseo-fasciati.

Sp. 6. Majuran.e Milli.

GG. Tarsi pnstici articulo basali nigricante. - Oculi hand grisen-fasciati.

$$
\text { Sp. 7. Ater Mgn. }
$$

FE. Abdomen partim nigrum, partim luteum ve rufum, vel rubescens.

11. Pedes femoribus rufis, apice luteis, hasiann distincle nec late nigricantibus.

Sp. 8. Ebracatce Mihi. 
HII. Pedes femoribus ounibus basi late nigricantibus.

I. Tibice posticie, anulo nigricante manifesto præditæ. K. Fascic ablominales testaceæ, omnes utua ab altera sejunclæ.

\section{Sp. 9. Quadrifasciatus Mgn.}

KK. Fascice abdominales rufæ vel rubesceules, aliquibus salteII in medio conjunctis.

Sp. 10. Lacerus Loëro.

11. Tibix postice anulo nigricante non manifesto.

L. Abdomen basi et apice nigrum; in medio rufum vel rubescens.

Sp. 11. Bicolor Fabr.

LL. Abdomen basi tanfum nigra, postice rufum.

Sp. 12. Testaceus Mgn.

(Nota) Il Paragus arcuatus del Meigen, stando alle rnazioni del Dot. Costa di Napoli trovasi in quel Regno: lo non l'ho mai ricevuto da nessuno de miei corrispondemi ue nazionali ne esteri, e non ho mai pututo raccoglierlo nell' llalia centrale, e quiudi non ho aruto mezzo di osservarlo, e perciò nemmeno di distingierlo sistematicamen!e, collocandolo nella serie delle Specie llaliane. - Non posso dir altro per ma riportandomi alle descrizioni degli autori, che essot fovrà collocarsi fra il Lacerus ed il Bicolor din min ordinamento, c sarà la trevicesina delle Specic: lla- 
liane di questo genere fino all oggi a me note. V. per questa specie la descrizione del Meigrelr.

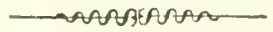

Observat. et Symonim.

Sp. 1. P. Coadunatus Rindn. (Annali Bologn: 1848).

Sic a me nuncupatus, quia speciebus pluribus auctorum in unica coadunatis comstal, scilicet.

H.euonkwots Higr. Nign. Macq.

CEneus Mgrl. Mgn. Macq. Zelt. Cosla.

Tibials Zelt. (Tibiis non anuulalis).

Tranguliferus Zell. - Sighlates Curlis.

Obscunus Mgrl. Meig. Macq. Walk. Cosla.

Tibialis Zelt. (libiis non annulalis).

Color abdominis in mare, grada!im ex nigro all rufum, in segmentis posterioribus varietatmo transit.

Quando istie abdomeu totum nigrtim prabent ut in fouminis, tunc p. obscurum Megerlei et Walkerii, et tibialem Zetterstedtii (tibiis non annulatis) representant.

Qhando in earum masculis, abtomen rufescit in medio tanImun segmenti tertii, p. Triangulifertum Zelterstedtii habemus, vel Sigillatum Curlisii: quando mares speciei abilonen apice tantum rufescentem probent, parago aneo Mrgerlei pertinent; et tablem quamlo tria segumenta apicalia tota rufa sunt, paragum Hemorrhomm abctormin denotant.

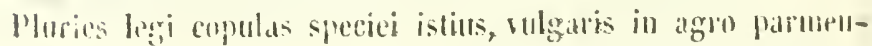


si lam in planilie quam in montursis, a menso aprilu ad netolresis, - In Loid Italia elitm commmonis, at

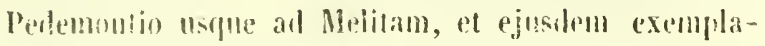
rid ommes antei enomologi misromul.

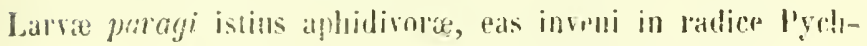

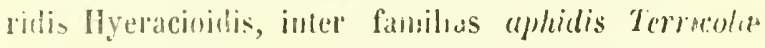
Renth, quas roral.

V. Observat: lleas, in Diariis (.nnali di Bulogua istaj).

Sp. 2. P. Renunates Mgrl. Mgn Wall.

V. Descript. Nieigetui.

Minima specierum nostratium, non rara in ditionis parmensis planilic el collibus, et magis frequens mensibus Junio et Julio. Lectus etiam in Etruria a D. I'iccioli.

Sp. 5. P. Tarsates Wihi. - Longit. Mill. 4.

Similis mari P'. Coadunali mei, abdomine toto atros et similis quoque femorato Megerlei, sed ab illo, sen a primo distinctus, tibiis late et manileste versis apicen nigricante-anulatis, el metatarsis poslicis stperne fusco-nigricantibus: ab isto vero, sell a fommoralo difert, magnituline semper duplo el ullis majore, nec non, lal'sis posticis hasi nigriean!e.

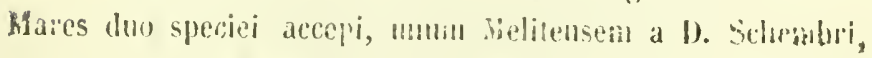
alterna Sicultum a Prof. Gemeilaro. 
Sp. 4. P'. I'siныou Mihi. - Longit. Mill. 5.

Magnitudine et habitu mari Thymiastri similis, sed Ancemee omnino nigræ. - Frons flavescens, prope alt!ennas fusca. - Facies flava, prominentia nigricante. - Thorax Scutellum et Abdomen atra, levia, scutello apice non albicante, pleuris fasciculis pilorum albo:um instructis.

Culiptera albicantia. - Halteres flavi.

Abdomen singulo segmento, vitlis lateralibus albo-tounentosis. Pedes flavescentes, fentoribus late ad basim nigris, posticis apice tantum lutescente: tibiis posticis anulo nigricante instructis: metalarsis posticis superne, anterioribus totis fusco-obscuris.

Marem unicum accepi a D. Pecchioli, prope Pisas ab eodem coptum.

Sp. 3. P. Thymastai Panz. Latr. Rndn.

Albifrons Fall. Mign. Macq. Loëw. Zett.

V. Descript. Zetlerstedtii.

Fiequens in agri parmensis planitie et collibus, a mense aprile ad septembrem. Vivit etian in Pedemontio ubi eum legit Bellardi, et in Insubria captus a Villa.

lijus larvæ aphidivo:æ. V. observat: meas in diariis (Nuovi annali di Bologna 1818.)

Sp. G. P. Majorane Mihi.-Longil. Mill. 4-3.

Sinilis P. Thymiastri sed satis distinctus, tibiis et carsis ommibus omnino luteis, dum constanter in al- 
tera specie tibiæ posticæ nigro-anulatæ, el metatarsi postici siltem superne nigricantes sunt.

Speciem hanc raro legi in agro parmensi: una vice etiam copulatam inveni.

Sp. 7. P. Aтеr Mgn. Macq.

v. Descript. Macquartii.

Species a me non observata: in Sicilia vivil teste Macquartio. Ab aliis specicbus præsertim distincta, fasciolis-tomenti grisei oculorum non manifestis.

Sp. 8. P. Ebracatus Mihi. - Longit. Mill. fere 6.

Similis habitu el magnitudine P. Quadrifasciato, at distinclissimus ab isto et ab aliis, characteribus istis:

(Fom.) Antenna elongatæ. - Facies flava, vitta nigra "1sque ad epistomium producta.

Thorax dorso antice, vitlis duabus griseis latiusculis, sulucontignis, ita ut unicam fere latissimam constituant. Abdomen segmentis secundo et tertio, basi fascia lutea, incisuris parallela, et contigua, integra, et lateribus dilatata: segmento guarto lateribus luteo-maculatis, et fasciola dorsuali pilorum albicantium: segmento quinto villis duabus alhidis in medio approximatis.

Pedes fere toti el ummino rufi, tibiarum et femorum apice tuteo-albillo: femoribus tantum qualuor anteriorilus ima basi niroricantibus. 
Alce vitta marginali luteo-fusca.

Fœminam tantum legi speciei, in planitie agri parmensis tempore autumnali.

Sp. 9. P. Quadrifasciatus Mgn. Macq. Rndn.

v. Descript. Meigenii.

Frequens in tota ditione parmensi, prasertim mensibus majo et junio. - Ejusdem exemplar obtinui a D. Schembri in Melita captum, et ab Insubria a Marietti.

Larvæ speciei ex aphidibus sonchi asperi vivunt.

V. observat: meas in diariis (Annali di Bologna 1848.)

Sp. 10. P. Lacerus Loëro. (Bemerkungen etc. 1\$40.)

Similis Bicolori at diversus, corpore minori, et tibiis posticis anulo nigricante manifesto.

Mares tantum quatuor, characteres hujus speciei præbentes, in agro parmensi inveni.

Sp. 11. P. Bicolor Fabr. Latr. Mgn. Macq. Zell. Rndn. Walk.

v. Descript. Zetterstedtii.

Vulgaris in agri parmensis planitie et montuosis ab aprile ad actobrem. In Etruria, in Insubria, in Perlemontio, et Liguria vivit, ubi lectum fuit a Pechioli, Villa, Bellardi, et Mussino 
Satis variat latitudine coloris rubescentis abdominalis, in fominis semper latioris.

Plures copulas hujus paragi etiam inveni.

Elus larvæ vivunt aphidibus, in radicibus herbarum habitantibus. - V. Observat. meas in diariis, (Nuovi annali di Bologna 1848.).

Sp. 12. P. Testaceus Mgn. Macq.

v. Descript, Meigenii.

Comunis in tota ditione Parmensi, præsertim tempore œstivo. Ex Insubria, ab Etruria, ab insula Melita, exemplaria ejusdem obtinui, a De Cristofori, Pecchioli, et Schembri.

Una vice marem Paragi hujus, cum fomina bicoloris copulatum inveni.

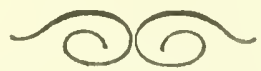

Genur 81. Psands Latr. Fabr. Mgn. Macq. Zett. Rndn. Walk.

Staphus $F a b r$.

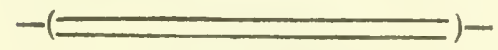

Cuaract. Gener.

Antennce capite transverso longiores; erectx, arliculo secundo, tertio longitudine subæquali, seu secundo elongato fere ut tertium. 
arista brevi crassiuscula subnuda, in parte apicali el dursuati articuli inscrta.

Frons in petiolum antenniferum, modice preducta.

Facies inferne subgibbosa, et supra excavata.

Ala fere ut in parago el pipizclla venosx, sed tamen diversx, vena transversaria exteriori magis distans ab interiori, ot satis extra apicem longitudinalis prime sita, nee non vena quinta longitudinali, cubito non, aut vix appendiculato.

Species Italica Sp. 1. Abdounalis Fabr.

Observat. et Synonim.

Sp. 4. P. Abdominalis Fabr. Latr. Mgn. Macq. Zelt. Rndin. W'alk.

V. Descript. Meigenii.

Speciej istius fomina tantum capta fuil a me, in colle subapennino ditionis Parmensis.

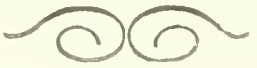




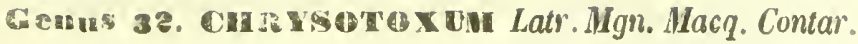
Zett. Loëw. Rndn. Walk.

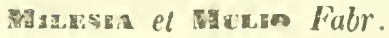
sumas liossi. Panz. Cusors Scop. - syasca. Lin.

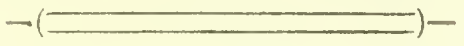

Chabact. Geneli.

Antenne capite transverso circiter atque longe, et erecta: articulo tertio elongato, eł etiam duobus primis magis vel minus longis: arish dorsuali nuda, prope basim articuli inserta.

Oculi pilusi vel pubescentes, iu froute maris late coherentes.

Facies subrecta, supra epistomium tuberculata.

Alas vena quarea luggitudinali extrinsecus manifesto sinuosa: transversa exteriore contra apicem primæ longitudinalis sita, quinta ad apicem, quartæ conjuncta coutra apicem tertiæ: spuria ordiuaria integra el perfecta, non interrupta.

Pedes validiusculi, sod non incrassati.

\section{SPecies ITALICE}

A. Fascia flava intermedia segmenti tertii abdominalis, latitudine subæcualis præcedente et sequente, seu non exilissima, et semper distincta.

B. Sculellum totum flavum, vix in medio paulo fusco-translucidun, sed inacula nulla nigra determinata. 
C. Abdomen limbo laterali toto flaro, seu totum flavi lintbatum.

\section{Sp. 1. Cisalpiyum Rndn.}

CC. Abdomen vel limbo laterali toto nigricante, vel partim flavo et partim nigro.

D. Antennœ articulo tertio satis elongalo, circiter bilongiore duobus primis conjunctim.

Ales macula fusca submarginali distincta.

Sp. 2. Italicua Rndr.

DD. Antennce articulo tertio non manifeste longiore duobus primis conjunctim, nisi breviore. - Ale haud fuscomaculatæ.

E. Antennarum articulus primus duplo circiter longior secundo. Abdomen limbo flavo postico segmentorum, in medio satis dilatate.

Sp. 3. Pahmense Rndn.

EE. Antennarum articuli duo primi longitudine subæquales.

- Abdomen limbo flavo postico segmentorum, in medio haud valde dilatato.

a. Fasciœ abdominis ad latera conjunclæ limbo flavo postico segmentorum. Alæ macula nulla subapicali.

Sp. 4. Fasclolatura De-Geer.

aa. Fascia flavæ ad latera distantes et non conjunctæ limbo flavo segmentorum. Alæ macula fusca sub apicali distincta.

Sp. 3. Coluner Mihi. 
BB. Scutellum non ounnino llavum, vel basi nigra margine llavo, vel basi flava apice nigricante, vel in medio macula nigra determinata distinctissima.

F. Scutellum basi anguste flava, postice late nigricans. Alco costa antica nigricante.

\section{Sp. 6. Marginatun Mgn.}

FF. Scutellum apice magis vel minus late flavo. Alce costa antica etiamsi lutescente vel fusca, tamen numquam nigricante.

G. Abdomen fasciis flavis intermediis segmentorum, usque ad limbum extremum lateralem productis.

1. Abdomen fasciis flavis intermediis segmentorum, ad latera connexis antice et postice, cum fasciis marginallbus flavis segmenti proprii et præcedentis: si vero a precedente sejunctis, tunc segmentis tertio et sequenlibus, postice totis et late flavo-marginatis.

\section{Sp. 7. Chrysopolita Rndn.}

HIII. 4bdomen fasciis flavis intermediis, vel omnino ad latera liberis, vel postice tantum, margini flavo connexis segmenti proprii. - Segmento secundo sepius, limbo navo postico nullo, vel lineola tantum, vel fasciola marginali exili, ut in tertio et quarto, maniista.

Sp. 8. Elegans Locho.

GG. Fasc $\infty$ flaræ intermediæ segmentorum, a lateribus abdoninis paulo remotæ. 
I. Antcnnarum articulus tertius, parum longior sccundo longitusculo. - Pedes femoribus omnibus etiam basi fulvis.

$$
\text { Sp. 9. Авсuтum Mgn. }
$$

II. Antennarum articulus tertins, fere bilongior secundo breviusculo. - Pedes femoribus quatuor anterioribus basi nigricante.

$$
\text { Sp. 10. Vernalis loëto. }
$$

AA. Fascia intermedia segmenti tertii abdominis, satis exilis, sublinearis, vel aliquando parum aut non manifesta.

K. Antennarum articulus secundlus, parum et non dluplo " ultra, primo et tertio brevior. - Abdomen fascia ittermedia segmenti quarti integra.

Sp. 10. Tricinctum lindn.

KK. Antennarum articulus secundus, duplo et ultra brerior præcedente et sequente. Abdomen fascia intermedia segmenti quarti in medio paulo interrupta.

Sp. 12. Bicinctum Lin

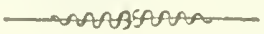

Observat. et Synonim.

Sp. 1. C. Cisalpinun Rndn.

V. Descrint, meam in Diariis (Annz), Socict. Entonol. Galliw 1845. 
Ab aliis speciebus prieserlim difert.

drtcunis articulu tritin, paulo longiore pracedentibns subxequalibus, conjunctim. - Acrlomine limbo tolo flavido vel lintw. - Alis margine antico, nsque all vemam secmidum bongitmdinaletil rufescente, et inter sermanlam et quartam obscure fusco. - fronte in firmina nigrial, fascia transversa, lata, integra, tlava: in mare antice nigra, postice fulva etc.

Bdibiar mon frequens in agri parmensis planitie et collibus, masertim mensihus majo el jumio.

Sp. 2. C. Italicum Rndn.

r. Descriqu, meam in Diariis s. c.

Species satis ab ommibus listiuctu, longitudiue articuh tertii anternartum, dupla dno:mu pracedentium conjunctim, præerer alios characteres. - Ab intermedio diversa, fasciis flavis, ad latera, segmento posteriori thetis; Alis, costa plus mimisve flavido-fusca; Scmello nom determinate maculato etc.

Frequens in tota litione Pamensi, ab aprile al octobrem.

- In Regno neapolitano (pnoque capins a .). Costa, in Insubria a fratribus Villa; et in V'encto a Com: Contarini.

Sp. 5. C. Paramense Rindn.

v. Descript. meam in Diariis s. c.

Species rarissimo capta a me in agro parmensi, cujus forminam tantum invenire polui. 
5p. 4. C. Fischolatum De-Geer. Mgn. Macq. Zelt. Mndh. Arcuaturi Panz. Walk. (esclusa Syn. Linnei). Sylvaruir Mgrl.

$$
\text { V. Descript. Zetterstedtii. }
$$

Itequens in agro parmensi, præsertim a mense aprile ad Julium. - In Pedemontio quoque non rarum, nam a Geneo, Mussino, et Bellardi, ejusılem exemplaria ibi capta obtinni, et sæpe etiam ab ltalia media et meridionali ab aliis amicis.

5p. 5. C. Colunum Mihi.

(Form.) Antennce articulis duobus primis subæque longis, tertio paulo longiore præcedente. Scutellum havum unicolor. - Fascice abdominis, a margine laterali distantes, et sejunctæ a limbo flaro postico segmentorum, isto in medio tantum segmentorum penultimi et pracedentis manifesto, et angusto. - Alce macula fusca distincta subapicali. - Pedes toti flavescentes, femoribus anticis et intermediis, non posticis, basi, nigricante.

formina lantum inventa, primo rere, in collibus ditionis Parmensis.

Sp. 6. C. Marginatem Mign. Loëio. Zett. Fasciolatumi Fall.

a Synom: Zetterstedtii, exclude Arcuatum Panzerii, quia istum Fasciolato De-Geerii pertinet.

V. Descript. Zelterstediii. 
In Pedemontio non infrequens, ejusdem exemplaria milni transmissa fuerunt a Mussino et Bellardi.

Sp. 7. C. Chrysopolta Rndn.

v. Descript: mean in Diariis s, c.

(Forle C. Octomaculati Curtis varietas).

Non infrequens in agen parmensi.

In Pedemontio quoque eum invenit Prof. Bellardi. Parmæ legitur præsertim primo vere, et sæpius in collibus.

Sp. 8. C. Elegans Loëw. Zelt. Rndn.

v. Descript. Zetlerstedtii.

(Mas.) Speciei a me semel inventus in agro parmersi, ejusdeın exemplar alterum ab Insubria misit Villa; feminam a Pedemotio accepi, ibi a Bellardi captam.

Sp. 9. C. Arcusuns Deg. Fabr. Fall. Mign. Macq. Contar. Zell. Loëw. Rndn.

Festivur Scop. Walk. (esclusa Syn. Limuei?)

v. Descript. Zetterstedtii.

Vulgaris in agro parmensi temupore verno et æstivo. A Pedemontio pluries eum accepi, et etiam ab aliis provinciis Italicis. 
30

\$j. 10. C. Veriale Loëw. Zall.

V. Decript. Zullersledtii.

Yulgarre, ctum pracedente inrenilur, in ditione Parmensi ct in ledemuntio, ubi sæye enm legit Bellardi. - In Liguria monute hubitat, ut in collectione Mussino obsarkari.

Olian specion hanc varietatem esse crelleban arcuati, antefluan characteribus autennamm stuterem, femorum basim engricantem concomilamibus.

4) 11. C. Tricheruy Rudn.

Bicıстии Kossi (pelibus flavis. Fauna Etrusca $1459)$.

Si, Ist. wlin a me distincta fuit, prasertim femorun colore unf fuls'seculs, sed nunc exemplaria alipua inveni fom-uibus basi magis vel ninus late el manifeste nigricuntidus, elian in forminis; ideo distinctionis causat fuicipua hujus Chrys: a Bicincto, mon in femoribus, sold in autemuis munc invenienda est, quia in Tricincto arliculus soumlas antennarum parmm brevior est pracedentr, ,lum in altero, anticulus iste duplo saltem lipuriol aliis.

Pli."era specimina plura Cr. Trincincti, omnia fascom flavam segmenti quarti latam, et perfecte integran prahent. et exemplaria duo fominea Bicincti, mihi tantum nota, fasciam hanc possident, in medio anguste ioterruptam, el si character iste constams, nptimus.

Fperiem istam el non sequentem dirersan esse pulavi a C. 
Bicincto auctorum, quia in hoc, femora semper hasi rufescenlia dicmulur, llum presertion in frominis Tricincri nostri, et etiam sapius in maribus, femora lola falrescentia sunt: et preterea in descriptione Bicincti, optima in opere Zelterstedtii, fasciam flavam segmmul। quarti, subintegram, ct non integram esse dicitur, farml non convenit Tricinclo meo, in fuo fascia ipsal semper integra olsservalur etc.

C. Tricinctum non rarum est in Parmensi ditione, ef legilu! eliam in Pedemontio el in Insmbria, ul rogmovi a speciminibus mihi a Bellarli et Villa missis.

Sp. 12. C. Bicinctuar Lin. Fabr. Fall. Mign. Macq. Com. loc̈ro. Lindn. Iralk.

r. Descript. Zelterstedtii.

Rarissimo legitur in agro parmensi.

A Pedemontio pjusden foeminam semel misit Bellardi (*)

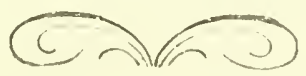

(") In Corsica D. Mann, et ad Tergestum D. Schiner, Chr. Scutellatum Macq. legerunt.

Species a me non observat, cujus descriptionem lege in npcribus Macquartii, et Italicis addenda. 
206

Stinps VI. MICRODONIN E Rndr.

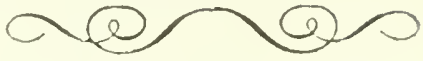

Geums 1. APriterTs Latr. Hacq. Cont Rndn.

wachodox Mgn. Fabr. Zetl. Walk. Curtis.

Srummes Fabr. Rossi.

Mucio Fab. Fall.

Stratomxs Panz. - Musca Lin. D.-Gr.

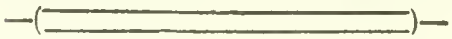

Cilaragt. Generici.

Antennx elongati, erectæ, basi subcontiguæ, apice divergentes, artioulo primo longiludine circiter duorum sequentium; secundo duplo et ultra breviore tertio.

Oculi nudi, in froute maris non :ontigui, sed paulo magis proximis oculis fæminæ.

Facies convexa, epistomio non porrecto.

Scutellum margine postico excavato, inde proninentias duas laterales exibeus.

Abdomen segmento quarto, auplo et ultra longiore tertio, præsertim in maribus.

Alce inter venas quartam et quintam longitudinales, veuula transversaria supranumerum, iu parte exteriori præditæ. - Venis ipsis longiludinalibus, extrinsecus, arcuatim et non angulatim flexis. Pedes rubusti, sed aon distincte incrassati. 
Species Italice.

A. Thorax et abdomen oenescentia.

B. Scutellum nigro-nenesceus.

Sp. 1. Apronuis $D e-G$.

BB. Scutellum testaceun.

Sp 2. Mutabilis Lin.

AA. Thorax cœrulescens: - abdomen violaceum.

- Sp. 3. Devius Lin.

Observat: et Synonim.

Sp. 1. A. Apiforus De-G. Rossi. Zett. Walk. Curtis.

Mutabilis Lin. Fabr. Fall. Mgn. Macq. Comt.

Migans Mign. Macq.

Conica Panz.

v. Descript. Zetterstedtii.

Sp. in apennino Parmensi legit non frequenter D. Bertens, in Pedemontio a Genèo el Bellardi inventa.

Sp. 2. A. Mutabilis Lin. Panz. Fall. Zelt. Rndn. Walk. Apiformis Mgn. Macq.

Apiarius Fabr. Cont.

v. Descript. Zetterstedtii.

In apeunino Parmensi raro leclus a D. Berléo el a me: el in Pedemuntio a Mussino et Bellardi inveulus. 
208

Sp. 5. A. Devirs Lin. - Piger. Sshin.

Species a me non observata. Virens prope Teigestum anl litora Allialici, teste Schinero.

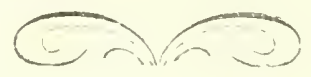

Stirps VII. CALLICERIN E kudn.

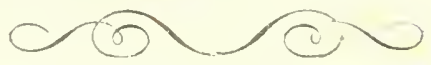

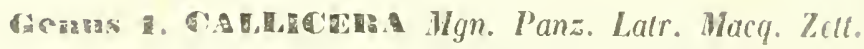
Rindn. Walk. Schuin.

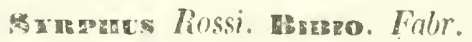

Chanact. Genehict.

Antcuno rapite Iransverso satis tongiores, in tuberculu frontali parum provlucto insertx; arista sen stilo apicali.

Oculi pilosn-subfisciati, saltem in maribus, in quibus superne late contigui.

Facies supra epistomium tuberculata.

A/re venis Inngitudinalibus quarta el guinta, in conjuнclione prope apicem, angulum interiurem sutis acutum efficientibus. Quarta non manifeste incurvata, nec senulam iransversariam suprannmorrun postice emiltente: trausicessaria exteriore magis proxi-

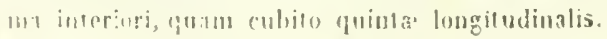


Abdomen propo basim non coarctatum.

Pedes robusti, sed fermoribus aullis, aec disctinete inorassatis, nee subtus spinulosis.

Corpus hirtum.

\section{Species Itakicre}

A. Abdomen non nigro-pilosum ad apicem, sed pilis fulvis omnio tectum.

B. Abdomen transversim nigro-fasciatum, vel nigro-viltalum - Thorax haud nigro-vittatus.

C. Abdomen segmentis secundo et terlio, postice fascia atra, integra, transversa, in utroque sexu distinctissima. Femora maris basi late nigricante, fomina tota fulvescentia.

\section{Sp. 1. Spinole Rindn.}

CC. Abdomen segmentis secundo et tertio, vittis duabus tantum nigris, lateralibus, transversis, distinclis. - Femora in utroque sexu, basi late nigricante.

$$
\text { Sp. 2. Rosen lindu. }
$$

LB. Aldomen nec nigro-fasciatum, nec vittatum. - Thorax vittis tribus longitudinalibus nigris, magis vel minus sed semper manifestis.

1). Antennœ articulis duobus primis, longitudine subacuali, et simul, ut tertius fere longis. - Oculi foemina subnudi.

$$
\text { Sp. 5. OExeA Fab. }
$$


DD. Ansennarum articulus pritnus fere bilongior secundo satis breve, et cum isto, satis et fere duplo breviores tertio. - Oculi in utroque sexu hirti.

\section{Sp. 4. Magquartil Rndn.}

Ah. Abdomen apice magis vel minus late nigro-hirtum.

B. Abdomen segmento oltimo tantum nigro-hirto sed toto.

- Tibice etiam apice omnino fulvæ.

Sp. ๖. Pornil Mihi.

EE. Abdomen segmentis duobus ultimis totis nigro-hirtis.

- Tibia apice nigricantes.

\section{Sp. 6. Bertolonir Miki.}

maAsianam

\section{Observat: et Synanimia}

Sp. 1. C. SpINol.æ Rndn.

V. Deseript. meam in Diariis (Annal. Soc: Eatom. Galliæ 18i4.) Frequens legitur in collibus subapenninis ditionis parmensis, rarius in planitie, mensibus septembre et octobre. Flores solidaginis diligit.

Sp. 2. C. Roserit Rndn.

v. Descript. in diariis \&. c.

Rara in agri parmensis collibus, temporibus et locis iisdem ubi prima species legitur, supra flores ejusdem solidaginis. 
Sp. 3. C. CEnea Fabr. Mgn. Latr. Macq. Zell. Rndn. Walk. (Panz. Sp. Dubia)

Aurata Rossi. Rndn.

v. Descript. Meigenii ot Zetterstedtii.

Species perrara in ditione parmensi, cujus mas semel captus fuit in apennino a D. Bertéo.

Sp. 4. C. Macquarti Rndn.

Rufa? Schum.

V. Descript: in Diariis s. c.

Dubitanter Rufæ Schumelii adscribo, nam diagnosis ejusdem auctoris mihi ignota.

In ditione parmensi non rara, mensibus septembre et oclobre, facilius tamen legenda in collibus subapenninis supra flores varios.

In Insubria quoque habitat ubi eam legit Comes D' Arco, et in Etruria inventa a D. Piccioli.

Sp. :. C. Porril Mihi.

Similis præcedentibus sed manifeste diversa.

A Calliceris Spinole et Roserii difert, abdomine nec nigrofasciato, nec vittato, et thorace vittis tribus nigris longitudinalibus distinctis: et a prima etiam femoribus, in utroque seru, basi late nigricantibus.

Ab Enea, articulis duobus primis antennarum, simul satis brevioribus tertio, et secundo distincte breviore priu1o, nec non oculis fominæ, ut in mare hirtis. 
Ab omnibus tandem distincta, abdominis segmento ultimo toto nigro-hirto.

Habitat rarissima in agro Parmensi, eam legi mense martio in floribus Buxi, et mense Aprile in floribus Grossularix, semper in locis collinis.

Anico dilecto Comiti Carolo Porro mediolanensi, malacologo Clarissimo, et entomologix amatori et cultori, anno 1848 occiso, species a me dicata.

Sp. 6. C. Bertolonı Mihi.

Similis Call. Porrii, sed ab ista et ab aliis satis distincta, abdomine segmentis tertio et quarto, seu duobus ultimis totis nigro-hirtis: Tibiis vero, præsertim anticis et posticis, apice nigricantibus; et colore minus œneo, sed nigriore, a C. Porrii difert: sed segmento penultimo abdominis nigro-hirto magis distat ab eadem, cui aliunde proxima.

Mas speciei semel $\mathfrak{a}$ me inventus in collibus subapenninis, mense majo, in foliis Lauri Cerasi.

Anico Prof. Josepho, Bertoloni, Bononiensi, Eutomologo et Botannico præstantissimo. Sp. hanc dedico.

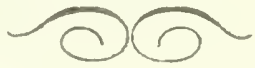

Gewus CegeIA Fubr. Mgn. Fall. Mlacq. Zelt. Rndu. Walk. Syspeng Panz. - Resca Lin.

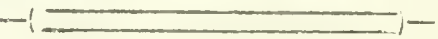


Chahact. Genek.

Antenute erecta, clavam elongatam constituentes, iu petiolo satis longo frontali insertæ: arista sen stilo apicali satis breve: petiolo longo circiter ut antennæ.

Oeuli uudi, in fronte maris late contigui.

Abdomen prope basim non distincte cosretatum.

Ale vena quarta longitudinali in parte exteriori intus manifeste dexa, et renulam postice emittente transpersariam: - Quarta cum quinta longitudinalibus, ad apicem, angulum interiorem acutum constituentibus. - Transversaria exteriore magis distante ab interiore, quam a cubito quintæ longitudinalis.

Pedes femoribus postieis paulo incrassatis, ef inferne aã apicem spiIIulosis.

lioryus subnudum.

\section{Spegies Italig.}

A. Scutellum basi flava, apice nigro.

Sp. 1. Conopsoines Lin.

A.1. Scutellum totum flavnm.

Sp. 2. Pespiformis Latr. 
Observat: et Synonim.

Sp. 1. C. Conopsoides Lin. Mgn. Latr. Maeq. Zett. Rndn. Cuntis. Walk. Costa A.

Craviconsis Fabr. Fall.

Conopsea Fall.

V. Dascript. Meigenii et Macquartii.

Vulgaris in tota Italia. Parms legitur a twense aprile ad julium. Ex Pedemontio, Insubria, Etruria, Neapoli, Sicilia, et Melita, ejusdem exemplaria ab amicis accepi

Sp. 2. C. Vespifonmis Latr. Mgn. Macq.

V. Descript. Meigenii.

Istius speciei exemplar in Sicilia captum a D. Ghigliani, Marchio Spinola mihi comunicavit, et ex Insula Melita D. Schembri exemplaria duo misit, et nnum ab Etruria D. Piccioli.

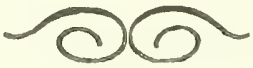

Cenux 3. Aphiximorpit Andn.

Cenen Mgn. Latr. Macq.

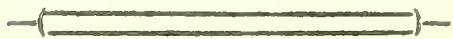

Chanlct. Generici.

Antonna in clarsm subfusiformem satis elongate, in tuberculo frontali parum producto, et non in petielo sayis longe inverts. 
Abdomen segraento secundo basi satis coarstato.

Catora circiter ut in gonerc Ceria.

\section{Spegies Italica}

A. Antenno apice nigro. - Scutellum basi flava apice nigro. - Abdomen nigrum margine postico segmentorum flavo.

$$
\text { Sp. 1. Subsrssiuis Fall. }
$$

AA. Antennoe totæ rufx. - Seutellum tolum flavum. Abdomon flavum fasciis et maculis nigris.

Sp. 2. Petronilu Rndn.

Observat. ef Synonim.

Sp. 1. S. Subsessius Fall. Mgn. Latr. Macq. Rndn.

r. Descript. Meigenii et Macquartii.

Satis raro Sp. hanc inveni in collibus ditionis parmensis primo vere, measibus martio et aprile. In Etruria quoque habitat ub! inventa a Doct. Pecchioli et mihi transmissa. 
216

Sp. 2. S. РеткоNILL Kandn.

V. Descript. mean in diariis ( sanal. Soc, antemolog: Gallik 1650.)

Fomina speciei a me semel capta in planitie prope-padana ditionis Paræansis, supra flores cujusdam Euphorbiæ, mense aprile.

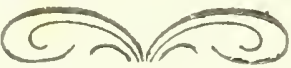




\section{FMUIMU.}

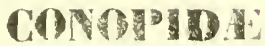

Cuanact. Faniziz.

Antennoe articulis tribus, (stilo vel arista exceptis) instructe, articulo tertio hats circulatim incisi. - Gence plus minusve sed distincte buccalie. - Oris apertura am pla, vibrissis destiluta. - Proboscis sæpius exilis at elongata, vel recta, vel ad medium cubitata et retro flexa, si raro brevis et crassiusculia, tunc antennip fusiformes, stilo brevissimo apicali. - Palpi parum perspicui. - Alarum vene longitudinales (axillaribus exceptis) septem raro, octo fere semper; quinta tantum extrinsecus flexa, et quartam apice petente; quando venæ septem, lunc maris anus, fimbriis longis, et fœminæ, lamina ensiformi præditus. - Caliptera parva. - Pedes el corpus totum, setis validis destitma 

Srips. VIII: CONOHIS Rimite

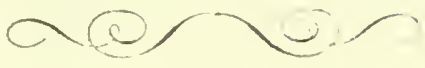

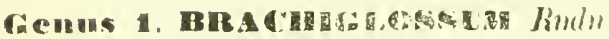

Lmoroldere Jinin. (oliui) $\left(^{*}\right)$

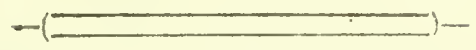

Charact. Generici.

Proboscis brevissima, crassiuscula, et fabiain. epistomium don superan. Abdomen segmento secudo, in utroque sexu, nou tistincte cuarctaltn Fenora subæqualia, von irregulariter in parte basali iantum incrassal a. Venter foeminx, theen infera no distincle producla (").

Srecies Italice

A. Ala margine anteriore non nigricante, etiamsi paulo firsciore.

B. Tarsi fere toti Iutei. - Frons antice el rerlice llava, fascia lala intermedia transversa nigra. - Pedes flavi, femoribus nullis nigro maculatis.

\section{Sp. 1. Diapematua Rndn.}

(1) Nomen hoc genericum immutavi, quia eutomologis non placurl.

(") Fominx et non mares, ut ereduat auctores, thecam ventraless prabent. V. Ohscrat. meas in Diariis (Annali di Rologna 1845. 
IBB. Fons tota nighta, velice tantuan lyavido, - Femorer postica, superne nıcula niģra signata

$$
\text { Sp. 2. Erostrature Rudn. }
$$

AA. Alte margine nigricante. - Tarsi fese toti nigri. Sp. 3. Calceatur Mihi.

(Not:ı) Alteram speciem a Windobona nuper misit D. Schiner, nomine Conopis Diademati Rndn. sed talis non est, $\therefore$ magis proxima B. Erostrato, et ab uno et altero Jiversa. - A Diademato difert, præsertim presentia Inacularum nigrarum, in femoribus posticis. - Fascia nigra frontali postica latiore, et margine exteriori recto, non dentato. - Abdominis segmento secundo §atis angustiore et latissime nigro; fasciis abdominis in segmentis 5, 4, 5, non interruptis etc. Ab Erostrato distincta. Fronte, fascia transversa flava prope apicem ornata, uon tota nigrı. - Abdominis segmento secundo valde angustiore; tarsorum articulo ultimo tantum, non duobus ultimis nigricantibus etc.

Sp. n. apellabilur B. Corenatum Mihi.

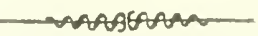



Observat. of Synonim.

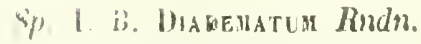

Y Descript. meam in diaris (Magazin de Zyotegie 1845.) 
Cuanth specien hanc crulgavi, dus lantm pjustem exeniplaria a me observata fuerunt, masculum unum $\mathrm{el} \mathrm{al-}$ lerum fomineum, iden modo incerto, characierem sixualem maris, fasciarum abdominis his interruptarum, tamquam stabilem indicavi; sed mon specimina plura possideo mascula, quı constanter fascias istas interruptas prabent, ideo chanater homus.

Non infrequens in cullibus dilionis parnessis, prope cavernas vespa vulgaris, mensibus provertin angnsto et

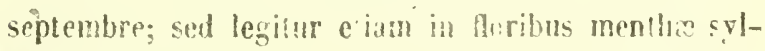
restris et aliis.

\section{Sp. 2. B. Erostratug Rndn.}

Y. Descript. meam in diaris (Annali di Eulogua $184 \mathrm{f}$ et Magasin de Zoolog: 18\$5.)

Species bene distincta a præcedente. Fronte in parte antica late flava, et femoribus posticis macnla nigricante supera.

"on rara in agri parmensis collibus, prope cavernas vulgaris, præsertim mense sepiembre.

Sp. 3. B. Calceatua Mihi. Longil. Mill. 9. (Fom.)

Antenna nigræ - Caput flavum, vilta faciali interrselli:, el macula frontali, antennarum basim late cingente, subquadrata, postice appeudice intermedia, ad verticem producta, nigris. - Thorax cum scutelio niger: utringue macula humerali el villa plemarnm flavis. - Abdomen 
nigrum, cingulis flavis tribus, ad marginem posticum segmentorum secundi tertii et quarti. = Halteres flavescentes. - Alce costa antica late nigricante, postice basim versus flavescente. - Pedes flari; anulo lato femorum, et tarsis, basi excepta rufescente, nigrris. - Capsula ventralis mediocris, non acuminat a. Semel capta foemina, mense septembre incipiente, in floribus menthæ silvestris, ad colles dit: Parmensis.

(Nota) Avendo trovato quasi sempre gli individui di queste Conopide in vicinanza delle abitazioni della vespa volgare, mi naque il sospetto che nei primi periodi di loro vita potessero abitare entro i nidi di quell'Imenottero, ma ora un tale dubbio è diventato cerlezza, perchè più di una volta gli ho colti sul falto, quando eltravano e sortivano dall' apertura che mette al ricovero delle sudette vespe; ed ho pure osservato che per entrare con sicurezza in quelle gallerie, colgono il monento, in cui nessuno di quegli abitanti può imbarazzargli la via.

Govremo credere perciò, stando alle regole di analogìa, che questi Ditteri nello stato di larva, divorino quelle dell' Inenottero col quale convivono, e probabilmente anche le ninfe del merdesimo.

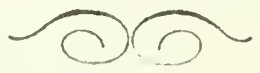


Genus 8. SPIxasouna Rindn.

Covors Fabr. Rossi. Fall. Mgn. Wiedm. Cur tis. Cont. Macq. Zett. Walk.

Empis part. Scop.

Covopicjes. Spazigzossum: Cmamonarmces Rndn. (olim).

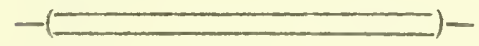

Charact. Gener.

Proboscis magis vel minus sed semper satis producta ultra epistostomium, sæpius exilis, et nou distincte ad apicem labiata.

Abdomen segmento secundo haud valde coarctato in utroque sesu. pedes femoribus omnibus plus minusve incrassatis, sed regulariter, et non in parte basali tantum; posticis non distincte crassioribus:

Venter fomina theca sub-apicali satis producla, vel acnminata rel rotundata.

Alir rirciter ut in Brachiglosso venusie.

\section{Species Italic}

A. Tarsi toti flavi vel rufi, aut ariculo apicali lantum nigricante.

B. Frons tota nigra, vertice tantum flavo.

a Fasciœ nigræ abdominis latæ, non dentalx, sed margine recto. - Facies nigro-villata.

Sp. 1. Quadmasciata D.-Geer. 


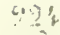

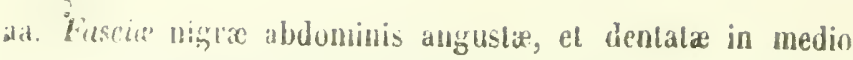
- Facies non nigro-viltata.

Sp. 2. Mucronata Mihi.

Bi. Frons fold, aut maxima parte flava.

C. Alo haul fusco-fasciatæ. - Antenne nigricantes etc.

Sp. 3. Aureifasciata Mihi.

C. Al A $\alpha$ fusco-fascialæ. -- Antenna ferrugineæ etc.

Sp. 4. Vescicularis Lin.

4. Tarsi toti vel fere toti nigricantes.

1). Femora omnia tola rufescentia.

Sp. 5. Flavifrons Mgn.

101). Fimora late nigro-fasciala.

Sp. 6. Flavipes Lin,

Observat: et Symonim:

à. 1 S. Quanufasgiata De-G. Fall. Lair. Mgn. Macq. Zetl. Rndn. Walk.

Aculeata? Fabr.

V. Descript, Zelterstedtii.

I Pedemontio unicum specimen misit olim Geneus; alterum postea accepi a D. Bremi Tigurino, et nuper dro ex.rnplaria a D. Schinero. - Numquam Parmie callus. 
Sp. 2. Mucronata Mithi.

Similis quadrifasciatæ, sed satis distineta, præsertim, Facie haud nigro-vittata, Abdomine cingulis nigris angustioribus, et postice in medio acuminato-prodictis, saltem in segmentis prope basim etc. - Abaliis vero conneneribus dirersa, Fronte tota atra (vertice excepto). - Antennis totis nigris. - Pedibus omnino flavis, vix apice tarsorum fusco. - Alis nec fusco-fasciatis, nec marginatis. - Scutello nigro. Fominæ theca ventralı non acuminata, sed sub-ovata etc Non infrequens in collibus subapenninis ditionis Parmensis, supra flores præsertim mentlæ sylvestris, tempore autumnali. - In Pedemontio quoque lecta a P. Bellardi.

Sp. 3. S. Aureifaschata Mihi. - Long. Mill. \%.

Maris Antennce nigræe, vix ad conjuncliones articulorum paulo ferrugininex.

Facies fulva, prope oculos et epistomium aurei micans. Frons rufescunte-fusca.

Proboseis breviuscula, sed non crassa, parum ultra epistominm producta.

Thorax niger, callis humerafibus et scutello fulvescentibus: villa transversa pleurarum, et macula lata metathoracis, ad unumquodque latus, amei micantibus.

Ilalteres et pedes fulvi; coxis nigris, aurei adspersis vel maculatis; tibiis posticis apice, et tarsorum posticorum basi, intus nigro-tomentosis. 
Abdomen segmento primo fere toto, secundo et tertio ad basim late, quato in medietate basali, quin to et sexto incisuris tratum nigris; alibi aurei nitens. Alce sublimpidx.

Mas speciei senel a me captus in collibus ditionis Parmensis, tempore autrmati.

Sp. 4. S. Vestreucares Lim. Fabr. Fall. Mgn. Macq. Zett. Fegregerge var? Macq. Muln.

V. Descript. Zettersts stii. eui alde,

Theca ventralis foeminz, valde producta, subacuminata.

Sp. rara in ditione parnensi, a me lecta in collibus primo vere.

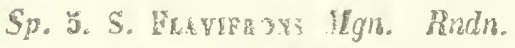

V. Descripr. Areigenii.

Rarissima apud nos, bis tantum ejusdem marem inveni in agra parmensi, et semel foeminam ab Etruria obtinui, a Piccioli missam.

Sp.6. S. Flutres Lin. Fabr. Rossi. Panz, Fall. Mgn. Macq. Zett. Iindn. Walk.

V. Descript. zelterstedtii, cui adde,

Foeminæ theca ventralis valde prolucta et sub acuminata. Quatuor tantum, specimina Italica hujus speciei possideo, 
ınum fomineum, lectum a Prof. Bellardi ad montem Rosa, alterum masculum, olim a D. De Cristofori mediolanensi missum, absque patriæ indicatione, forte in alpibus Insubriæ inventum; et duo ad Tergestum capta a D. Schinero.

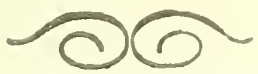

X'scars 3. CONorituA Rndn. (Magaz. de Zoologie 1845). Covors Mgrl. Meig. Walk.

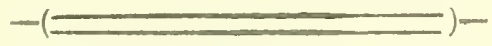

Charact. Gener.

pipoboscis exilis et satis producta altra epistomium.

Abilomen segmento secundo haud manifeste coarctato: ventre in fremina, theca copulatoria non producta.

femora jmaia regulariter incrassata, posticis distincte crassioribus, et in parte apicali canaliculo infero basim tibiarum continente Alce ut in generibus affinibus eirciter venosa. 
226

Species Italica Sp. 1. Cerieforis Mgrl.

Observat: et Synonim.

Sp. 1. C. Cerieformis Mgrl. Mgr. Rndn. Walk. (exclusa Synon. Macquartii. )

V. Descript. Meigenii.

Sp. apud nos rarissima; ejusdem foeminam semel inveni in collibus ditionis parmensis. Ab Etruria masculum misit D. Piccioli.

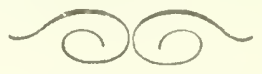

Cenus 4. WONoms Fabr. Rossi. Fall. Mgn. Wiedm. Macq. Zett. Rndn. Walk. Costa. Emers part. Scop.

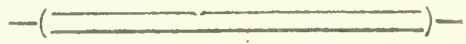

Characteres Generici.

Proboscis satis exilis et elongata.

Abdomen segmento secundo satis coarctato, prasertim in maribus, aliquando subfiliforme.

pedes femoribus omnibus, in mediclatc basali tantum, irregulariter incrassatis. 
Venter furmina, theca copulatoria salis producta el sub acuninata. Ale ut in generibus afluibus circiter renosa.

\section{Species Italice}

A. Halteres capituli lateribus spinulosis.

$$
\text { Sp. 1. Semiatra O. Cosla. }
$$

AA. ITalteres capilulo non spinuloso.

B. Pedes, femoribus omnibus, in parte saltem apicali rufescentibus.

C. Frons in medio niglo-villata.

D. Femora basi nigra, vel anulo nigricante.

$$
\text { Sp. 2. Macrocephala Lin. }
$$

DD. Femora omnino rufescentia.

E. Antennæ magis vel minus rufescentes, saltem rufo-maculater.

$$
\text { Sp. 5. Rufires Falr. }
$$

EE. Antennce tolæ nigræ.

$$
\text { Sp. 4. Meridionalis Macq. }
$$

C. Frons haud nigro-villata, sel omnino flava.

F. $A l \propto$, in parte anteriori, inter venam costalem et primam longitudinalem decolores, etiamsi circum nigricautes.

$$
\text { Sp. ร. Vittata Fabr. }
$$


230

FF. Ala margine autico toto nigricante, etiam inter venam longitudinalem primam et costalem.

$$
\text { Sp. G. Lacera Mgrl. }
$$

BB. Femora ommia, vel saltem postica, in parte apicali nigricanlia.

G. Frons flava.

$$
\text { Sp. 7. Pusilla Mgn. }
$$

GG. Frons nigricans.

$$
\text { Sp. 8. Puinla Macq. }
$$

Ouserv: er Symonim.

Sp. 1. C. Sementa Costa.

V. Descript, in dirtis (Amali dell Accademia dulle scienze di Napati 1836.

Species a me thin non observata: lecta a Prof. Costa in insula Procida; sed nmper marem ejusdem accepi a D. Schiner, nomine C. Viltala mas. - \& quo difert, non solum spirulis halterum, sed etiam femoribus posticis in medietste apicali intus nigricantibus, nacula fusca in dorso segmenti secundi abdominis etc. ad Tergestme capium

Sp. 2. C. Mracnogernaea Lin. Fabr. Rossi. Mgn. Fall. Hacq. Zetr. Rntn. Walk. V. Descript. Zettersteltii. 
Hujus speciei unicum exeruplar pussiatu, ignotat originis, dubitanter igitur Italicis adscripta, et forte varietas est C. rufipedis, femoribus basi infuscata: eum tamen in Firniria habitare Rossins affirmat.

Sp. 3. C. Rufures Fabr. Rossi. Fall. Mgn. Macq. Zett. Walk. v. Descript. Zetterstedtii.

Species von vulgaris in agro parmensi, sed non rarissima, præsertim in montuosis, tempore æstivo. Ejusdem exemylaria Insubrica a Fr. Villa, Etrusca a Pecchioli, Pedemontana a Bellardi, Ligustica a Nussino accepi.

Sp. 4. C. Meridionalis Macq.

V. Descript. ejusdem Auct.

Species in Sicilia capta, teste Macquartio, et a me non obserrata.

Sp. 3. C. Vitrata Fabr. Latr. Mgn. Macq. Zett. Rndn. v. Descript. Zetterstedtii.

Species sat frequens in agro parmense, ubi legitur a mense majo ad A ugustum. Habitit quoque non rara in aliis mrovinciis Italicis, testibus Prof. Bellardi, Mussino, D'Areo, Pecchioli et Schembri.

Sp. 6. C. Lacera Mgr. Mleig. Macq. Rndn. v. Descript. Meignanii. 
Rara in ditione parmensi, legitur præesertim mense junio. In Sicilia quoque vivit, ubi ean legit Ghigliani, et exemplar ab eodem captum, dono March. Spinoli obtinui. - In Etururia lecta a D. Piccioli.

Sp. 7. C. Pusilla MIgn. Nacq. Rndn.

v. Descript. Meigenii.

Species non rara in agro parmensi, ubi tempore cestivo legitur. In Etruria etíam inventa a Doct. Pecchioli.

Sp. 8. C. Pugila Macq.

V. Ejusd. Auct. descript.

Semel species ista a me inventa fuit in collibus parmensibus, et semel a D. Pecchioli prope Pisas.

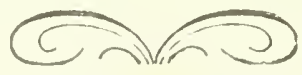

StIRDS IX. ZODIONIN E Rnin.

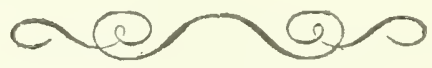

Cenu: 1. Todiov Latr. Afgn. Desv. Mlacq. Zelt. Rndn. Walk.

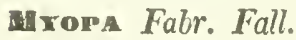

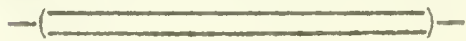


Charact. Gener.

Aantenne capite transverso breviores; arista dorsuali, brevi, nuda, in medietate apicali articuli inscrta.

Proboscis exilis, longa, non bicubitata.

Oculi nudi, subæque distantes in utroque sexu.

Abdomen subtus incurratum; fomine ventre theea copulatoria satis porrecta præùito, sed terebra anali ensiforme uon instructo; maris ano, appendicibus nullis filiformibus manifestis.

Alce venis longitudinalibus quarta et quinta, ad apicem, prope costalem conjunctis; septima et octava pari nodo ad apicem cosjunctis, sed longe a margine postico alarum, unica tantum I!mbum attingente.

Pedes femoribus crassis, præsertim anterioribus.

\section{Species Italie}

A. Antennce partim rufæ et partim nigræ.

Sp. 1. Cinereum Fabr.

AA. Antonna tolæ nigræ.

Sp. 2. Notatur Mgn. 


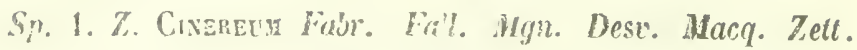
Rundr. Walk.

Coxopsnors Latr. - Ticmus Fobr.

Notatuin Walk.

v. Descript. Zetterstedtii。

C, nntinissimus in agro parmensi, a majo ad octobrem. imtividua pedemontana hujus speciei a P. Bellardi, etrusca a Piccioli, insubrica a Villa, et corsicana a March. Spincla accepi.

Sp. 2. Z. Notatui Mgn. Desv. Macq. Zett. Irnorata el Tessellata Fabr.

v. Descript. Macquartii.

cui adde, non solum antennis nigris omnino, a præceveute distinctus. sed etiam colore corporis obscuriore, et pedum nigriore.

Cum specie Cienererm labitat, minus frequens, in agro parmensi.

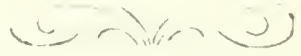




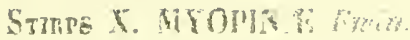

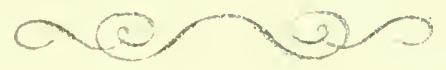

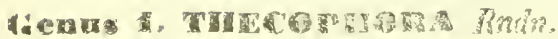

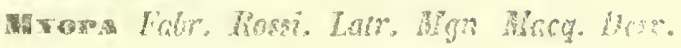
Zeft. Finds. Walk.

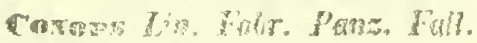

\$йеs: Serp:

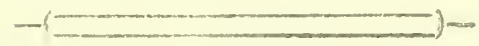

CHAPACT, GaERERE.

Antenna isulo circier longiores distantia oculortm ab apice froutis earam articales secundus satis longior tertio: arista nudo, bre= vis, is medio eircites dorsi artieuli tertii sita.

Proboscis satis exilis, bienbisata, parte infera fostice Lexa, longitudine supere 3 mbequali.

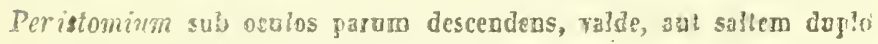
brevius, ocalis soperpositis.

Abdomen maris appendicibus analibus fillformibus destifutum; focminae oviducto longo non instructam, sed lteea rentrali satis producta.

the renis longitudinalibes gazasta et quinta sejonetim costali probluetis: septima ch netava conjunctis satis longe n margine posti=

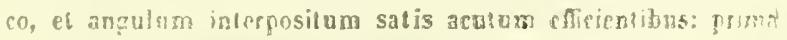
semispuria cusn secunda ad apicem in costali cosfoza. 
236

Species Italice

A. Fomora postica, saltem basi fulva vel testacea, - Frons late nigricans in parte posteriori et verticali. B. Femora postica tota fulvescentia. - Thorax dorso villis tribus cinereis, longitudinalibus, intermediis.

Sp. 1. Distincta Wiedm.

BB. Femora postica ad apicem magis vel minus late nigricanlia. - Thorax dorso vittis duabus tantun longitudinalibus, cinereis, distinclis.

C. Femora postica parte basali late fulvescentia. - Antennarum articulus secundus satis longior lertio.

$$
\text { Sp. 2. Atra Fabr. }
$$

CC. Femora postica ima basi tantum fulvescentia. - Antennarum articulus secundus longitudine fere oquali tertio.

$$
\text { Sp. 3. Pusilla Mgrl. }
$$

AA. Femora postica omnino etiam basi nigra. - Frons lutea et fulva, vertice tantum nigricante.

Sp. 4. Melanopa Mihi.

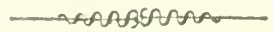

Observat: et Synonimia

Sp. 1. T. Distincta Wiedm. Mgn. Zett.

V. Descript. Zeltersledtii, cui adde

Theen ventralis fomine, nen valde producta, lata, et non acuminata. 
Non rarissima in agri parmensis collibus.

Maris hujus exemplar, a Regno Neapolitano, D. Costa mihi transmisit.

Sp. 2. T. Atra Fabr. Rossi. Panz. Fall. Mgn. Macq. Zelt. (esclusa Synonim: Pusizze Mgrl.) Rndn. Walk. (esclusa Synnn: eadem).

Fulvipes Desv. Macq. Mgn.

v. Descript, Zellerstedtii.

Sp. comunissima in dilione parmensi, tam in planilie quam in monttosis, a mense majo ad septembrem. In Pedemonlio Bellardi, in Insubria Villa, in Etruria Pecchioli (et Possins olim), in Insula Melita Schembri eam legerunt.

Sp. 3. T. Pusilla Mrgrl. Mlgn.

v. Descript. Meigenii.

Satis distincta a 'T. præcedente, non solum colore temurum posticorum vix ima basi testacea, sed præsertimbrevitate secundi articuli antennarum, vix aut non longioris tertio, et etram magnituline salis ninore, ila ut maxima istius exemularia, minores sint grandiorun T. Altro.

Non infrequens cum præcedente, in agro parmensi legitur, el in appennino quoque inventa. 
a3

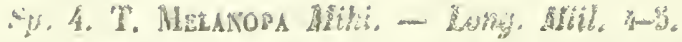

"com. Similis ilerum atru, sed ab eadem satis distincta, nt ab alis congenerilus.

Śbtemaram arliculis secundo et tertio longiludine subaqualibus; sucundo inferne, et tertio basi rmescentibus. Fronte lola fulva, antice lutescente, el verice exmtum nigro. - Orbita aculorun abs. - Fatio in paste

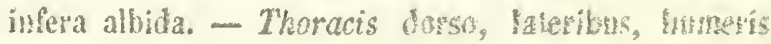

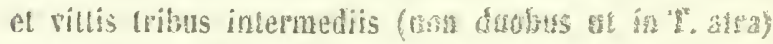
cinereis. - Hedibus nigris, genenis angustissme,

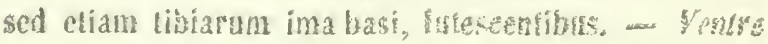

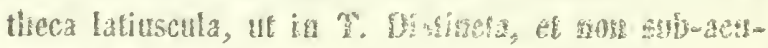
minata ut ig $\mathrm{T}$. atla.

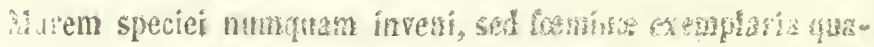

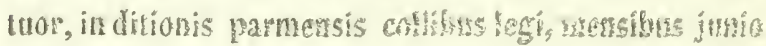
el jittio.

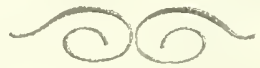

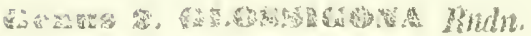

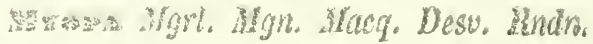

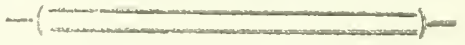

Chincteres Gromig?.

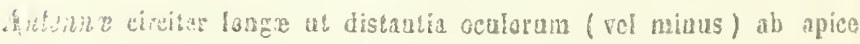
frontis: articulo secunilo batitudine E'stiacte minore tertio. Lista ut in Thecophora. 
Peristomium inferne descendens circiter ut longitudo oculorum, genis sub oculos non manifeste barbatis.

Proboscis satis cxilis et elongata, ab origine ad cubitum exteriorem circiter duplo longior capite.

Theca ventralis fominæ porrecta, sæpius oviducto apicali coujuecta, et tunc parum distinguenda.

Coteri characteres circiter ut in generibas proximis.

SPECIES ITALICA Sp. 1. Nupta Mihi.

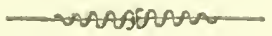

Observat. et Synonim.

Sp. 1. G. Nurta Rndn.

Mlas: Bicolor Mgrl. Mgn. Macq. Desv.

Foem. Nigra Mgn. Mlacq. Desv. Rndn.

In agro parmensi species non frequenter lecta.

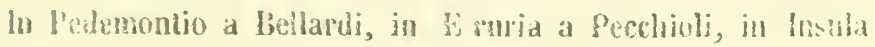
Metita a Schenbri etian inemtu.

Aula. Qtiattinque non siano mai stati colti in accoppiamento gli individui delle due Glossigone Bicolor e Nigra, ture per la somiglianza fra loro ne prineipali caralteri specifici, e per la circostanza di nou aver mai lrosalo dello femine cui conrengano tulte le secon:- 
darie marche distintive della Bicolor, e nessun maa schio che possa essere considerato per quello della Nigra, siamo costretti a credere che ambitue appartengano ad una specic sola; ragione per cui ho creduto di dover abolire i due nomi che dislinguevano le due specie supposte direrse, sostituendone uno che denoti la loro congiunzione in una sola specie.

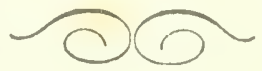

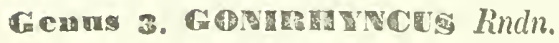

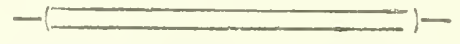

Cuaract. Genes.

Antennce breves circiter ut distautia oculorum ab apice frontis: articulo secundo longo et lato, fere ut tertius: Arista ut in generibus præcedentibus.

Oculi parum lateraliter elongati.

Peristomium sub oculos descendens circiter ut iculorum longitudo, genis non distincte barbatis.

Proboscis brevissima, cubito exteriori non producto ultra epistomium Venter feminæe theca copulatoria nos producta.

Pedes fenoribus incrassatis, at inferne serrulatis. Cœtera ut in generibus affinibus. 
Obscrvat: et Synonim:

Sp. 1. G. Dispar Rindn. - Longit. Mill. 5.

Olim credilli Sp. hanc Occultam esse Wiedmani, sed nunc in certitudinem veni, distinctam esse ab eadem, et novam: nam in diagnosibus auclorum Sp. Occella, nemo loquitur de brevitate extraordinaria proboscidis, sed tantum de longitndine minori partis inferæ: et nemo, verba dicit de colore diverso abdominis in uno scail, dum in nostra Sp. segmenta secundum et tertium cjusdem, lufescenlia sunt in mare (Femina Auctorum ). Sed tarsis etiam basi tantum, et non totis rufescentibis, dispar mea, ab occulta distincta est.

I'ræterea accuratissimus descriptor Zetlerstedlius, antennarum articulun! secundum bilongiorem esse tertio, in Occulta dicil, qnod Dispari non convenit, in qua articuli isti suberquales sunt.

lueo tamiquam novam eam describo.

Antennce filsco-rufescentes, aut testaceæ.

Caput albidı-Intescens, fronte nigricante, ad anicem anguste lutea.

Jraboscis brevissina, cujus pars infera paulo brevior superiore.

Thorax niger, Lumerisel lateribus paulo albicantibus. Abdomen fusco-rufescens, segmento primo toto, tertio ot 


\section{2}

quarto in medio nigricantibus: secundo tertio et quarto ad latera, quinto et sexto totis, albido-pollinosis. Halteres lutei.

Pedes nigri, tibiis basi lutescentibus, saltem extrinsecus: tarsis basi rufescente.

Llæ pailo infuscatæ, basi pallide luleæ.

Fœm: difert: Fronte antice lutea, postice fulva. -- Abdomine nigro, non rubescente, lateribus et margine postico segmentorum albicante.

Præterea oviductum præbet atrum, leve, sub ventrc flexum. Satis raro uterque sexus speciei a me lectus, in agri parmensis collibus.

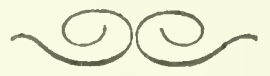

Genme 4. My QPA Fabr. Rossi. Latr. Meig. Fall. Masq. Desv. Zett. Rndn. Walk.

Conops Lin. Fabr. - Sicus Scop.

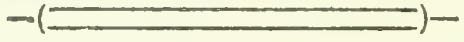

Ceraract. Generici.

Antenne breves, ad summum longæe ut distantia oculorum ab apice Prontis, earum articulus secundus sepius distincte longior tertio, aut raro subæqualis, et tunc latior.

Oculi breves, peristomio ad latera satis breviores, et raro fere longilengitudine æquali.

Proboscis longa vel longissima, semper epistomium magis vol minus superans. 
Venter fueminx, theea copulatoria non porrecta.

Pedes validiusculi, sed femoribus raro paulo incrassatie, ot rarissimo distincle subtus serralatis.

Cotera ut in generibus aftinibus.

Species ITALiCE

A. Alce nec fust:0-fasciat $æ$, nec maculatæ, nec punctatx.

B. Abdominis segmentum secundum distincte longius tertio.

- Geno nec barbatæ, nec distincte pilosæ.

C. Femora crassa sed non crassissima, et subtus non manifeste serrulata. - Thorax dorso rufo, vittis tribus nigris.

Sp. 1. Ferruginea Lin.

CC. Femora valde crassa, et inferne manifeste serrulata. Thorax dorso toto nigricante.

\section{Sp. 2. DOAsamis Fabr.}

BB. Abdomen segmentis secundo et tertio subœque longis. - Genæ distincte piligeræ, nisi barbalæ.

D. Antennarum articulus secundus satis longior tertio. Os margine nigro-bipunctato.

Sp. כ. Variegata Fall.

DD. Antennce articulis secundo et tertio longitudine sub rqualibus. - Os margine haud bipunctato. Sp. 1. Ponula Mihi. 


\section{4}

AA. Ala vel fusco-fasciatæ, vel maculatæ, aut saltem venula transversaria anteriore fusco-marginata.

E. Gence sub oculos, nec antice nec postice, nigro-punctatæ.

F. Vena transversaria tantum anterior fusco-marginata. Scutelium nigricans.

\section{Sp. 5. Stygma Mgrl.}

FF. Fena transversa exterior, et aliæ venæ, fusco-marginatæ, vel maculatæ. - Sculcllum ferrugineun.

G. Macula nigricans unica, ad venulam transversariam anteriorem; colel' venæ, fuscedine tantum cinctæ.

Sp. 6. 'lestacen Fabr.

GG. Maculœ Digricantes tres, una ad renulam transversam anteriorem, alter'a ad apicem venæ sextæ lungitudinalis ubi conjungitur quinta: tertia minus determinata, ad cubilum vemæ sextæ.

\section{Sp. 7. Polstygma Mihi.}

EE. Genæ, antice el postice, vel saltem postice, punclis nigricantibus notata, sub oculos.

H. Genœ, punctis nigricantibus antice el poslice signatæ. Alce macula fusca notata, determinata, inter renas longitudinales quartam el quintam, el ad renam iransversam interiorem.

$$
\text { Sp. 8. Picta Panz. }
$$

HH. Gence, postice tantum, puncto aliquo nigricante notalæ. Alœ macula nuia fusca determinala, nec ad ad renám transve:sam interioren, nec intra renas longitudinales quartam et quintam.

Sp. 9. Buccata Lin, 


\section{5}

Ouservat: el Synonim.

Sp. 1. M. Ferruginea Lin. Fabr. Latr. Fall. Meig. Macq. Zell. Walk. (esclus. Synonim. Dorsalis Testacea el Grandis).

V. Descript. Zettersledtii.

Satis raro sp. ista a me capta fuit, in ditionis parmensis collibus, et in apennino: etiam ab Etruria semel a D. Pecchioli spec. accepi.

Sp. 2. M. Dorsalis Fabr。Fall. Mgn. Desv. Macq. Euphippiuar Fabr. - Testacea Rossi. Fasciata Mign.

v. Descripl. Zettersteátii.

Raro a me inventa in agro Parmensi, et ralı a Prof. Bellardi in Pedernontio. - Habitat etiam in Etruria, teste Rossio.

Sp. 5. H. Vartegata Fabr. Fall. Mgn. Desv. Macq. Zelt. Nitidula Fabr.

v. Descript. Zellerstedlii.

Ex Pedemontio a Bellardi specimina Myopæ hujus mihi transmissa.

Sp. 4. M. Peelea Mihi. - Longit. Mill. pere 3. Morı? Mgn. - Nana? Desv. Macq.

Antennarum articulus secundus paulo brevior tertio; primus et tertii apex nigricantes, secundus fere totus, et tertii basis rufescentes. 
Frons nigro-pilosa, antice flara, in medio rufa, postice ut vertex fusco-ferruginea.

Facies et Gence albicantes, istæ albo-pilosæ.

Proboscis exilis, sed brevis, parum longior capite transverso, et parum ultra epistomium producta.

Thorax ut sculellum niger, nigro-pilosus, humeris et lateribus, paulo cinerascentibus.

Abdomen nigrum, nigro-pilosum, segmentis intermediis præsertim ad latera, postice cinerascentibus.

Caliptera cum halteribus albicantia.

Pedes, femoribus nigricantibus, geniculis et basi rufescentibus, posticis ad basim latius rufis: Tibiis ut tarsi fusco rufescentibus, anulis duubus fuscioribus, magis vel minus perspicuis.

Alæ paulo fuscescentes, basi pallidissime lutea.

Sp. Non comunis in agro parmensi: in floribus præsertim bellidis perennis, mensibus Aprile et Majo legitur.

( Nota) Diagnosibus incompletis Meigenii, Desvoidii, el Macquartii, nemo credere postest, speciem istam, vel $\mathbf{M o -}$ rioni, vel Nanæe referendam esse, nec scire, si illa Meigenii, cum ista Desvoidii confundenda sit.

Certe nemo de characteribus antennarum præcipuis, de brevitate proboscidis, de anulis tibiarum, de rufescentia basali femorum etc. verba dixit; ideo M. Puellam non descriptam esse retinendum est.

Sp. ॐ. M. Stygma? Mgrl. Meig. v. Doscript. Meigenii, cui adde. 
Ablomen naris, lateribus magis vel minus manifeste rufomaculatis: femora ut tibiœ, apice et basi fusco-anulata in utroque sexir.

Raro legilur in collibus agri parmensis, supra flores varios pratenses, primo vere, præsertim mense aprile.

(Nota) Si characteres anulornm, in femoribus et tibiis maris et fœminæ, et coloris abdominis in mare rufomaculati, in vera M. Stygma Megerlei non adsunt, tunc specimina ista Parmensia, Myopæ novæ referenda, quæ apellanda erit $\boldsymbol{M}$. punctum Mihi.

Sp. 6. M. Testacea Lin. Fabr. Latr. Fall. Mgn. Desv. Macq. Zett. Rndn. Walk.

Ferrugines Panz.

v. Descript. Zetterstedtii.

Vulgaris in agro parmensi, a majo ad Junium. - Lecta fuit quoque in Pedemontio a Bellardi, in Etruria a Pecchioli, in Insubria a Galeazzi, in Insula Melita a Schembri, et ejusdem exemplaria corsicana mihi communicavit M. Spinola.

Sp. 7. M. Polstygua Mihi. - Longit. Mill. fere 6.

Similis varietatibus aliquibus M. Testaceæ, a quibus diversa tantum, piclura alarum, quæ in nostra, maculas plures nigricantes, determinatas præbent: Istarum, una in venula transversaria anteriori observatur, altera ad apicem renx sexlx longitudinalis, ubi conjungitur 
quinta; tertia minus nigra ad cubilum venæ sexlı: altera ubi venæ longitudinales quarta et q̊uimı marginem attingunt, lata et diffusa in limbo adest. Prælerea ad apicem areolæ posticæ ultimæ, a venis constitutæ septima et octava, puncti duo nigricantes manifesli sunt.

Characteres isti, quamvis in unico exemplari Parmæ capto observati, tamen tanti pretii mihi videntur, ut speciem ab aliis distinctam denotent.

Sp. 8. M. Buccata Lin. Scop. Fabr. Panz. Fall. Heig. Macq. Zctt. Walk.

v. Descript. Zetterstedtii.

Spec. frequens in agro parmensi. In Pedemontio eliam capta a Prof. Bellardi.

Sp. 9. M. Picta Panz. Mgn. Desv. Macq.

Buccita Rossi.

v. Descript. Meigenii.

Sp. non infrequens in agri parmensis planitie et collibus, mensibus aprile et majo. - In Etruria quoque habitat, ubi eam legit olim Rossius.

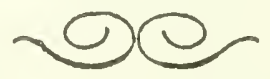




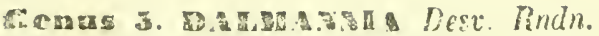

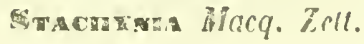

ragera Fabr. Lalr. Fall. Mign.

STo:goxsg Fabr.

Cuandet. Gexentci

Antenno magis vel minus longiores distontia neularum a! apico fron. tis: ariculis secundo et l.rio, longitudine param diversa.

Proboscis exilissimn et longissima.

Dculi satis losgiores productione infera peristomii.

Abdoman ano maris, appendicibus longis fliformbus; foeminæ ovidu. cto ensiformi, sub ventre flexo instruetum.

Tarsi pulvillis et uncis apicalibus, in ulrogue seru subrque longir. Ale areola anali, seu ultima postica, satis breve, el apice iu angulara scutum non producta.

\section{Srecies Italiga.}

A. Aloe venis Iongiludinalihus fpritia el quarta conjunclis, noo contra apicem arenle analis, sel propius basi.

B. Abdomen maris, segmenlis intermediis qualuor-maculalis. 
macnlis duabus dorsualibus, et duabus lateralibusfieminir, late Iransversin nigro-fascialis.

\section{Sp. 1. Desponsata Mihi.}

BB. Ablomen in utroque sexu, segmentis intermediis nigrotrimaculatis.

\section{Sp. 2. I'unctata Iabr.}

A.. Ala venis longitudinalibus terlia et quarta conjunclis contra, nisi exlra apicem, areola analis.

C. Ablomen uriusque sexus, in me lio dorsi llavum, niggropunctalum. - Scutellum apice llavo.

$$
\text { Sp. J. Flavescens Mgn. }
$$

CC. Ablomen vel tolum nigrlum, vel incisuris aliquibus sega mentorum, anguste flaridis.

Sp. 4. Parvilu Milio.

Observat. et Synomim.

Sp. 1. S. Desponsata Mili.

Mas: Gemans Wiedin. Mgn. Desv. Macq.

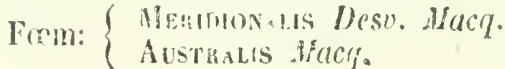

Sprecies ton rara in agri parmensis cullibus et montwosis, prosertim mensibus aprile et majo. - A me elian bis empulata inventa. 
Bom Mlussino eam in Liguria legril, Com. Contanini Veneliis, et Prof. Gemellaro in Sictlia. (*)

Sp. 2. S. Punctata Falr, Lalr. Foll. Mgn. Hacq. Zell. Rudn. Virexs Mlyn

v. Descript. Zelterstedtii.

Sp. Commnis in dilione parmensi, lam in phanilie quam in montihns, mensihus aprite et majo. - Lxemplar ejnsdem siculum, a D. Cosia Neapolitano accepii.

Sp. 3. S. Flavescens M!gn. Macq.

v. Deseript. Meigrnii

Sn. Non rara in agri parnemsis planilie ef collibus, ubi lew gilur, mensibus aprile el majo.

Sp. 4. S. PARveled Mihi.

Mas. $\left\{\begin{array}{l}\text { Marianata Mlgn. } \\ \text { Makgialed Zell. }\end{array}\right.$

Form: Flavipes Desv. Macr.

Species tres anctorwn sexus diversos pt varietales credo uniex speciei, que opinio confirmata est ab obser-

(1) Le non poche varield dei due sessi di questa specic, c gli accoppiamenti dei medesini da me racen!li ed osservali, mi hauno assisurato, che to Stachiniu Gemina Austratis c Yeridionalis, apparlengono ad una sola specie, di cui sono od $i$ stosi durerentı, o varteta, jer eui furmo con essa la Specio cuora, ehe nommat Desponsata. 
252

vatione speciminum nonnullorum in ditione nostra a me inventorum, ideo nomine comuni specifico eas nuncupavi.

Non frequens, primo vere; in ditionis parmensis collibus a me lecta.

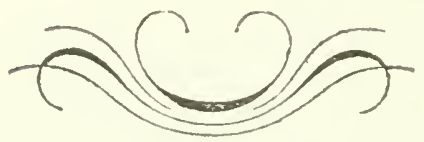




\section{IN DEX.}

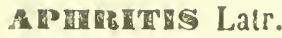

Pag. 206

$\Lambda$ piformis DeGeer.

Mutabilis Lin.

Asera Mgrl.

Enea Mğn.

Dispar Mgn.

Podagrica Fabr.

Quadrifasciata Mgn.

ED Ce Fabr.

- 104

Elongata Fabr.

Obscuripennis Mgn.

Sculellata $\operatorname{lgn}$.

PER A C A

Calceallim Rindn.

Coronatum Rindn.

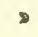

- 100

?

7

3

$>$

-

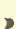

D

s

220

Diademalma Rindn.

Erostral:iur lindn.

H马.

Al'chaid I'zl',

Bicolor Fall.

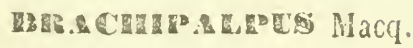
Olivacens Wiedm. 
2:3\%

Valgus l'anz.

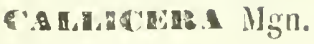

Pag. 78

Enea Fabr.

Berlolonii Rndn.

Macquartii Rndn.

Forrij Rondu.

Roserii Rndn.

Spinola Rudn.

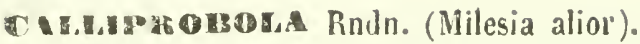

72

Fallax Lin.

Speciosa Rss.

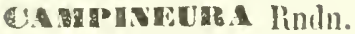

105

Tenusta Rindn.

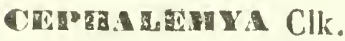

Ovis Lin.

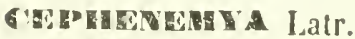

25

Carea Fabr.

212

Conopsoides Lin.

Vespiformis Latr.

Cuelzorhyous Rndn. V. G. Sphixosoma

223

cracitosid Mgrl.

Aurata Fahr.

Comacina zelt.

Flariconis Fahr.

flavimana Mgur.

Flasipes l'z.

Fiaterna Hon.

Gluilla Rudn.

Gorosid Fall.

turmplat Kell. 
253

Laticornis Rndu.

Iatifions Zetl.

Nigripes Mgn.

Estracea Lin.

Pedemontana Bell.

Precox Zell.

Pusilla Rudn.

Sculcliata Mgn.

Soror Zett. .

Subalpina Rndn.

Variabilis Fall.

Vernalis Fall.

Conopers Rndn. V. G. Sphixnsoma

COrondzes Rndn. (Conops alior)

227

Ceriæformis MIgrl.

covops Fabr.

228

Lacera Mğll.

Macrocephala Lin.

Meridionalis Macr.

Pumila Mgn.

Pusilla Macı.

Rufipes Fabr.

Semiatra Costa. 0.

Villata Fabr.

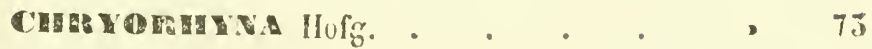

A picata Mgn.

Berlherina Fabr.

Brebissonii Macı.

Femorata Lin.

floccosa $M \lg 1$. 
1)i

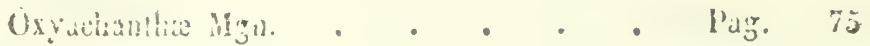

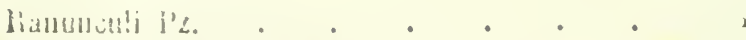

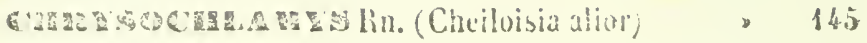
surea lindu.

Cillpiea Scoll.

linficurmis Fabr.

e :

Ceneteriorum Lin.

Chälybeatus Mgn.

Nubilis find.

Siplendens Mgn.

'larsatus Mgr!.

Vidualus Lin.

CEa Arcualum Lin.

Bicinclum Lin.

Cisalpinum lindn.

Chrysopolita Rondn.

Elegans Loew.

Fasciolatum D-Gr.

Jarsubricum Mihi.

Jalicum Rndn.

Marginalum Ngn.

Parmense lindn.

śsuitlalum Macq.

bricinctum Puda.

Veruatis I :

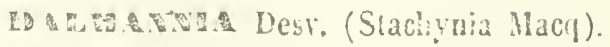

249

Brespunadia Lindo.

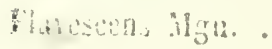


Parvula Rudn

Pag. 250

Punctata Fabr.

Dibra Macq. (Enica Mgn). . . . , 46

Alneti Fall.

Fasciata Maco.

DOADS Mgn. . . . . . . , 108

Conopsens Fabr.

Enıca Mgn. V. G. Didea, . . . •

Enterourysa V. G. Estrus. . . . ,

$\$ 0$

Eristalinus Rndn. V. G. Eristalis.

CIBIST TLIS Fabr. .

38

Aneus Scpl.

Arbustorum Fabr.

Intricarius Ln.

Nemorum Ln.

Orticola De-G.

Pascuorum Rndn. (pratorum al).

Pulcriceps Mgn.

Rupiun Fabr.

Saltum Rndn.

Sepulcralis Lin.

Similis FII.

Tenax Lin.

Eristalouya Rndn. V. G. Eristalis. . . , 40

EUMAIR US Mgn.

83

Alpinus Bllrd.

Angusticornis Rndn.

Barbarus IVdm.

Barbiventris Rudn.

Bicolor Bell.

Clavitibius Rndn.

Delicatæ Sclımb.

Exilipes Rndn.

fiuneralis Mgu.

Impudicus Rndn. 
Ornatus Mgn.

Sabulonum Fll.

Strigatus Fll.

Tricolor Fbr.

Truquii Rndn.

Tuberculatus Rodn.

Uncipes Rnd.

Exochesla Rndn. V. G. Brachiopa.

Ferdinandéa Rndn. V. G. Chrysochlamys. Gasterophilus Walk. V. G. Estrus. Gastromysa Rindn. V. G. Astrus.

Gastrus Mgn. V. G. Astrus.

Guossucora Rndo. (Myopa alior).

238

Nupta Rndn.

GONRER ReUS Rndn.

Dispar Rndn.

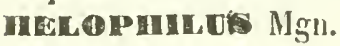

Lineatus Fbr.

Pendulus Lin.

Peregrinus Loëw.

Solitarius Rndn.

Trivittatus Fabr.

HEIEIVGe Rndn. (Pipiza alior).

Zetterstedtii Rndn.

IN YODERA Clk.

Bovis Fabr.

TA SIOPRTHECU Rndr. (Syrphus alior).

$$
\text { p }
$$

$$
\text { b }
$$

$$
\text { D }
$$$$
\text { b }
$$$$
\text { D }
$$

$$
\text { D }
$$

$$
\text { D }
$$
Albostriatus Fll. Bellardii Pndn. Coronatus Rndn. Dignotus Rndn. Gemellarii Rndn. Glaucus Fabr. Lucorum Lu. 
Novtis Rndn.

Pyrastri Lin.

Seleniticus Mgnt.

Topiarius Mgn.

HEdOES Mihi.

Viltata Mgn.

Lejogaster Rndn. V. G. Chrysogaster.

Lejota Rndn. V. G. Psilota.

Leopoluius Rndn. V. G: Brachiğlossum.

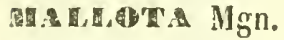

55

Fuciformis Fabr.

Melanogaster Rulu. V. G. Chrysogaster

166

Melitureptes Lw. V. G. Sphærophoria.

MERPBON Latr.

51

Aneus Mgrl.

Areus Rindn.

Armipes Rndn.

Bulbortum Rndn.

Cinereus Fbr.

Clavipes Fbr.

Funestus Fbr.

Italicus Rndn.

34

Montanus Rndn.

Mucronatus Rndn.

Nigritarsis Rndn.

P'ulicus Bell.

Rufitibius. Rndn.

Senilis Mgn.

Sicanus Rndn.

Spinipes Fbr.

Subfasciatus Rindn.

Submetallicus Rindo.

'Tuherculalus Rndn.

Varius Rndu.

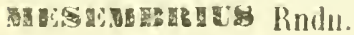


Pcregrinus Loèr.

Microdon V. G. Aphritis.

Pag.

MILESIA Ltr.

Saltuum Fabr.

MYATROPA Rndn. (Eristalis alior)

Florea J.n.

MYOPA Fabr.

Buccala Lin.

Dorsalis Fbr.

Ferruginea Ln.

Picta Pnz.

Polistygma Rndn.

Puella Rndn.

Punclum Rndn.

Stygma Mgrl.

Testacea Fabr.

Variegata Fll.

OEdenagena Clk.

22

GeSTRE L Lin.

Equi Clk. . . . . . . "

Ferruginatus Zett. . . . . ”

Hæmorrhoidalis Ln. . . . . "

ORTHONEURA Nacq. . . . . "

162

Nobilis Fall.

PA ne.

Arcualus Mgn.

Ater Mgn.

Bicolor Fabr.

Coadunatus Rndn.

Ebracatus Rndn.

Femoratus Mgrl. .

Lacerus Lw.

Majoranæ Rndn.

Pechiolii Rndn.

Quadrifasciatus Mgnn. 
Tarsatus Rniln.

Teslaceus Mgn.

Thymiastri Fll.

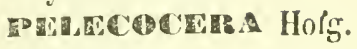

116

Tricincla Sig.

IPIEa Fall.

Excalceata Rindn.

Festiva Mgn.

Lugubris Fabr.

Noctiluca Lin.

Obscura Maq.

Signata Mgnn.

Vidua Rndn.

Vitripennis $M \mathrm{gn}$.

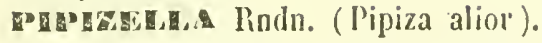

Varians Rindn.

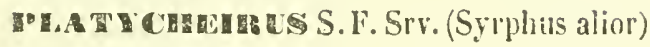
Albinanus Fabr. Clypeatus Mgn. Fulviventris Maq. Manicatus Mgn. Parnatus Rndn. Scutalus Mgn. Spathulatus Rindn.

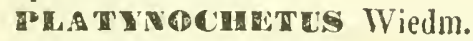
Selosus Fabr. Pegared Latr.

Abdominalis Fabr. rsar.ora Mgn.

Conjugata Rndn. Inunpta Riniln.

Campestris Mgn.

Rostrata Scop. 


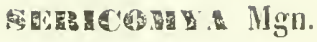

lag.

26

Bombiformis Fall.

I,apyona Lin.

SPARTASTERE Rndu.

106

Apenninus Rndı.

Lugubris A. Costa

Spaziglossuy Ruln. V. G. Splixosoma

22.5

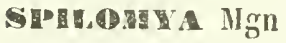

Vespiformis Mgn. (V. Milesia)

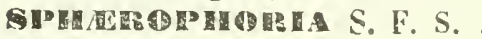

Melissæ Mgu.

Menlhaslri Ln.

Nigricoxa Zelt.

Oleandri Schmb.

Picla Mgu.

Scripta Lin.

Serpilli Rindu.

'Tæniala Mgn.

SERISGIVA Mgu.

Clunipes Fal!.

Nigra Mgn.

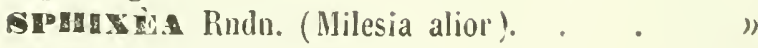

68

Crabroniformis Fabr. . . . . »

Splendida Rossi. . . . . . ”

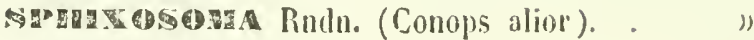

Aurifasciata Rndn.

Flavifions Mgn.

Wlavipes Ln.

Mucronala Rudn.

Quadrifasciala D, Geer. . . . . "

Tescicularis L. . . . . . . \#

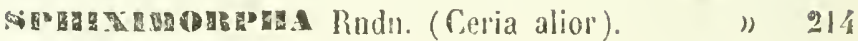

J'enronillat kindn.

Subsessilis Fll.

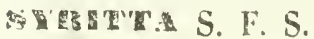


Pipicus Liu.

Sy

Auricollis Migi?.

Balliheatus 1), Gr. . . . . "

Bifiscialus Fbr. . • . . "

Bucculaius lindo. . . . . . )

Cinctus Mgn. . . . . . . "

Corollæ Fbr'. . . . . . . "

Decorns Mgn. . . . . . . "

hives Rinln. . . . . . . .

Pestivus For. . . . . . "

Flaviceps Rndn. . . . . . . "

Gracilis Jgn. . . . . . "

líyalinatus Fll, . . . . . n

l.umiger Mğn. . . . . . . "

Maculicornis Zell. . . . . . "

Mellinus L. . . . . . . "

Nigritibius Rnılı. . . . . . n

Nilibicollis Myn. . . . . . \#

Ornalus Hyu. . . . . . . .

libiliesii Lill. . . . . . . "

Fiosaltu Flos, . . . . . . " "

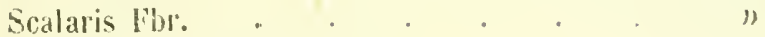

Umbellatarum Falle. . . . . . "

Vilripennis Mgn. . . . . . \#

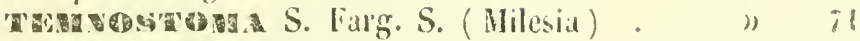

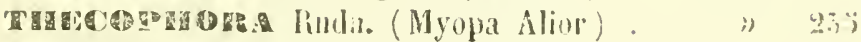

Alra Falı!.

bistincta Wirolim.

Mejanopa Pindn.

I'usilla Mgrl.

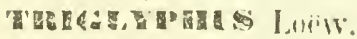

P'rimus l,i.

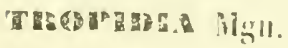

Fasciala Yirn. 


\section{4}

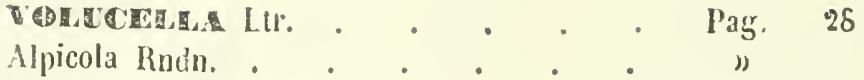
Bifasciala Scp. . . . . . . ״

Bombylans Lin. . • • • • • " •

Dryaphila Scpl. . . . . . .

Jnanis lin. . . . . . . "

Myslacea Lin. . . . . . . "

Pellucens Ln. . . . . . ״

Proxima Rndn. . . . . . . 》

Xy. . . . . . . . . .

Abiens Wilm.

Florum Fab.

Ignava Pz.

L.enla Mgn.

Nemorum Fbr.

Segnis Ln.

Sylvarum Ln.

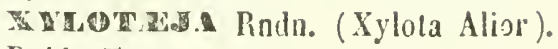

Dubia Fabr.

Vara Fabl.

20.

Cymbiciformis Fall.

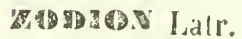

Cinereum Fbr.

Nolalum Ms̆n.

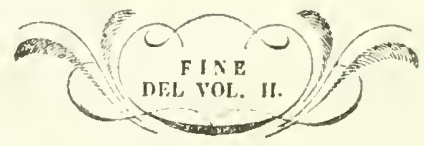


ICON, IN SYRPHINORUNH ALIS,

YEXARTY ET AREOLARUI OISTINCTIONEN EXPLANAXS.

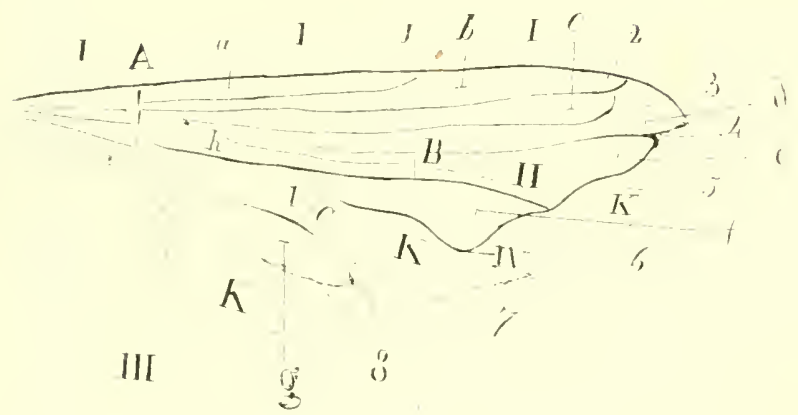

1. Tena Costalis.

II. - Spuria supranumerum.

III. - Axillaris.

IV. - Appendicularis, rel appendicula cubitalis.

1. - Longitudinalis prima - 2. secunda - 3. tertia - 4 . quarta etc.

A. Venula transwersa radicalis

B. - - anterior, vel prima

C. - - posterior, vel secunda.

a. Areola exterior prima - b. secunda - c. tertia - d. quarta - c. quinta - f. sexta.

g. - analis, vel axillaris, seu ultima

1. - basilaris, vol interior prima

i. - - - - secunda.

k. Spatium marginale: vel margo posticus alæ.

DIPTER. ITAL. PRODR. T. II. - 1857. 






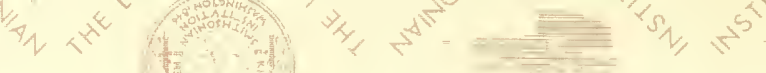

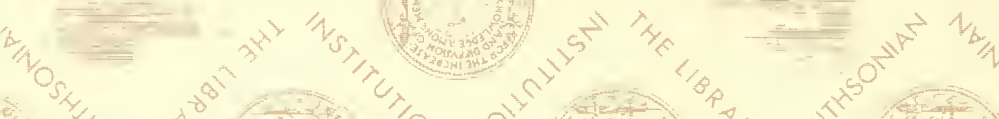

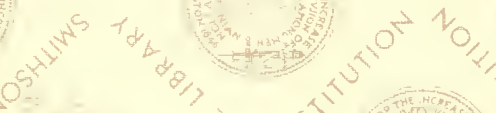

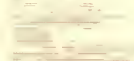

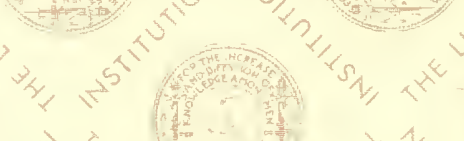

. $\mathrm{O}_{1} \mathrm{~s}^{3^{3}}$<smiles>C1=CC2CCCCC(=C1)CCC2</smiles>

$e^{+2} 1$ $8^{2}$ $\left(\begin{array}{ll}2+5 \\ 2\end{array}\right.$

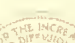

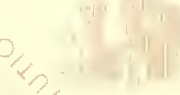

(1)

$s_{0}$

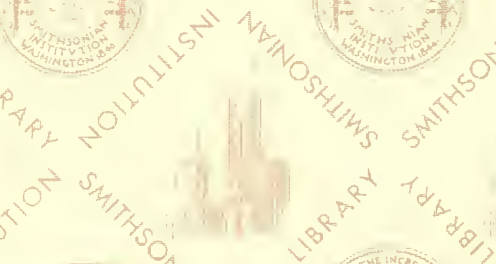

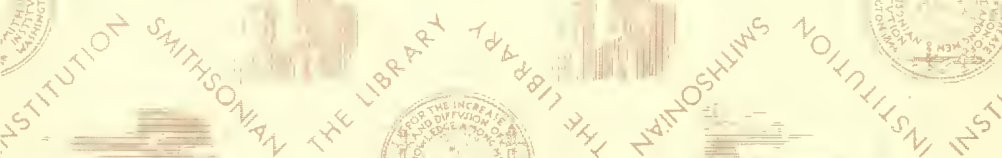
$\frac{b}{2} / c$

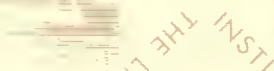

(1)

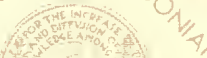

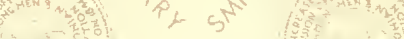

(a)

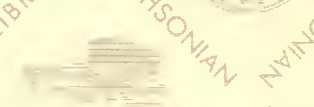


QL

535.4

I $8 \mathrm{R} 77$

v. 1-2

Ent.
Rondani, Camillo, 1807. 1879.

Dipterologiae Italicae.

QL

535.4

I $8 R 77$

v. 1-2

Ent. 
